DOE/NASA/0138-1

NASA CR-165206

Exinended Development of a Sodium Hyolroxide Thermal Energy Sirorage Module

Richard E Rice and Peter E Rowny

Comstock \& Wescott, Inc

and

Barry M Cohen

Consultant

Jul. :"11 1301

September 1980

Prepared for

NATIONAL AERONAUTICS AND SPACE ADMINISTRATION

Lewis Research Center

Under Contract DEN 3-138

for

U.S. DEPARTMENT OF ENERGY

Conservation and Solar Energy

Division of Energy Storage Systems 
NOTICE

This report was prepared to document work sponsored by the United States Government. Nelther the United States nor 1 ts agent, the United States Department of Energy, nor any Federal employees, nor any of their contractors, subcontractors or their employees, makes any warranty. express or lmplied, or assumes any legal liability or responsiblilty for the accuracy, completeness, or usefulness of any information, apparatus, product or process disclosed, or represents that its use would not infringe privately owned rights. 
DOE/NASA/0138-1

NASA CR-165206

\section{Extended Development of a Sodium Hydroxide Thermal Energy Storage Module}

Richard E. Rice and Peter E Rowny

Comstock \& Wescott, Inc

Cambridge, Massachusetts 02238

and

Barry M. Cohen

Newton Centre, Massachusetts 02159

September 1980

Prepared for

National Aeronautics and Space Administration

Lewis Research Center

Cleveland, Ohio 44135

Under Contract DEN 3-138

for

U.S. DEPARTMENT OF ENERGY

Conservation and Solar Energy

Division of Energy Storage Systems

Washington, D.C. 20545

Under Interagency Agreement DE-Al01-77ET26945

N81-26556\# 



\section{FOREWORD}

The program reported herein is a continuation of prior work under Contract NAS3-20615 from the NASA Lewis Research Center reported in NASA CR-159465 in December 1978. Both programs were carried out by the same principal personnel. However, Barry M. Cohen, who was a member of the Comstock \& Wescott staff during the prior program, has acted as an independent consultant to Comstock \& Wescott during the present program. 

TABLE OF CONTENTS

SUMMARY . . . . . . . . . . . . . . . . . 1

INTRODUCTION . . . . . . . . . . . . . 3

Background . . . . . . . . . . . . . . . 3

Present Program . . . . . . . . . . . 4

PART I - ANALYSIS OF THERMKEEP SAMPLES AND

EXAMINATION OF HEAT EXCHANGER . . . . . . . . . . . 7

Chemical Analysis of Thermkeep Samples . . . . 7

Examination of Heat Exchanger . . . . . . . 8

Heat Transfer Fluid . . . . . . . . . . 8

Chemical Analysis of Thermkeep . . . . . . . 9

Discussion of Analytical Results.... . . . . Il

Chemistry of Thermkeep-Steel System . . . . . 15

Summary of Chemical Analyses of Thermkeep . . . 21

PART II - VERIFICATION OF COMPUTER MODEL

BY EXPERIMENTAL DATA . . . . . . . . . . . . . 23

Introduction . . . . . . . . . . . . . . 23

Heat Transfer Fluid . . . . . . . . . . 23

Experimental Model . . . . . . . . . . 24

Preparation of Test Unit . . . . . . . . 25

Testing Procedure . . . . . . . . . . . 26

Enthalpy-Temperature of Thermkeep . . . . . . 27

Hysteres is Study .. . . . . . . . . . . 29

Data Correlation ........... . . 32

Summary. . . . . . . . . . . . 37

PART III - DUAL HEAT EXCHANGE DESIGN ANALYSIS . . . 39

Introduction . . . . . . . . . . . . . . . . 39

Approach ................ 39

General Features ............ . 41

Heat Exchange Analysis . . . . . . . . . 44

Description ............ . 44

Governing Equations . . . . . . . . 47

Application . . . . . . . . . . 49

Performance Analysis . . . . . . . . . . 51

Results of Computer-Model-Predicted

TESS Designs.... . . . . . . . . 53

CONCLUSIONS . . . . . . . . . . . . . 67 
$\begin{array}{lllllllllllllll}A & P & P & E & N & D & I & X & A\end{array}$

THERMKEEP POST TEST ANALYSIS . . . . . . . . A-1/A-6

(Skinner \& Sherman, Inc. Technical Report)

A $P$ P

HYSTERESIS STUDY GRAPHS . . . . . . . . B-I/B-20

$\begin{array}{lllllllllllllllllll}A & P & P & E & N & D & I & X & C\end{array}$

DATA CORRELATIONS

A $P$ P $P$ E $N$ N D I X $\quad$ D

USE OF COMPUTER PROGRAM . . . . . . . . . . . . D-1

Input Data . . . . . . . . . . . . . . D-1

Input Data Selection... . . . . . . D-4

Output Data . . . . . . . . . . . D-7

COMPUTER CODE ................. D-13

LIST OF SYMBOLS FOR MAIN PROGRAM . . . . . . D-33

LIST OF SYMBOLS FOR SUBROUTINE ELANAL . . . . . D-35

$\begin{array}{lllllllllllllll}\text { A } & P & P & E & N & D & I & X & E\end{array}$

SINGLE HEAT EXCHANGER COMPUTER CODE . . . . . E-1/E-19

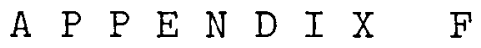

REFERENCES •. . . . . . . . . . . . . . . F-1 
TABLE I . . . . . . . . . . . . . . . . . 10 Analysis of Thermkeep from TES Vessel, and Original Material

TABLE II . . . . . . . . . . . . . . . 30 Test Conditions for Hysteresis Study Correlation

TABLE III . . . . . . . . . . . . . . . 35

Tests with Caloria HT-43 under Present Program

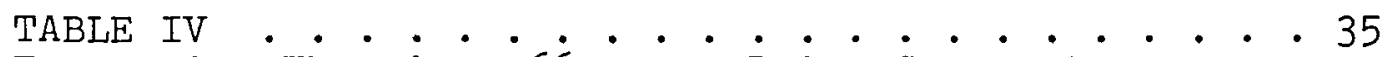

Tests with Therminol-66 under Prior Contract

TABLE V ..................... 61 Characteristics of Reference Design

TABLE VI . . . . . . . . . . . . . . . . 64 Comparison of Three Methods of Calculating Heat

Exchanger Cost for Dual Heat Exchanger TESS

TABLE VII . . . . . . . . . . . . . . . . 65 Per Cent of Total Cost of TESS

TABLE VIII . . . . . . . . . . . . . . . 66 Summary of Dual and Single Heat Exchanger TESS Design Points 


\section{LIST OF FIGURES}

Figure 1 . . . . . . . . . . . . . . . . . . . 12 Variation of $\mathrm{NaNO}_{3}, \mathrm{Na}_{2} \mathrm{CO}_{3}$ and $\mathrm{NaCl}$ with

vertical location in tank.

Figure 2 . . . . . . . . . . . . . . . . . 13

Variation of $\mathrm{MnO}_{2}$ with vertical location in tank

Figure 3 . . . . . . . . . . . . . . . . . 14

Variation of $\mathrm{NaNO}_{2}$ with vertical location in tank

Figure 4 . . . . . . . . . . . . . . . . . . 17

Variation of $\mathrm{NaOH} / \mathrm{NaNO}_{3}$ ratio with vertical
location in tank.

Figure 5... . . . . . . . . . . . . 18

Phase diagram, $\mathrm{NaOH}-\mathrm{NaNO}_{3}$

Figure 6 . . . . . . . . . . . . . . . . 28

Temperature-Enthalpy tables for Thermkeep

Figure 7 . . . . . . . . . . . . • . . . . 34

Typical solar daily cycle at Sandia Total Energy

Test Facility

Figure 8 . . . . . . . . . . . . . . . . 42

Solar electric power generating system schematic

Figure 9. . . . . . . . . . . . . . . . 46

Element for heat exchange analysis

Figure 10 . . . . . . . . . . . . . . . . 55

Heat transfer fluid return temperature vs.

charge time

Figure 11 . . . . . . . . . . . . . . . 57

Specific cost vs. Figure of Merit for various

heat exchanger areas

Figure 12 . . . . . . . . . . . . . . . 58

Specific cost vs. Figure of Merit for various

Thermkeep masses

Figure 13 . . . . . . . . . . . . . . . . . . 59

Specific cost vs. heat exchanger area for various

Figures of Merit

Figure 14 . . . . . . . . . . . . . . . . 60

Specific cost vs. Thermkeep mass for various

Figures of Merit 


\section{LIST OF FIGURES}

\section{APPENDIX B}

Figures B-1 through B-18. . . . . . . B-3-B-20 Thermkeep temperature vs axial distance

Test No. 1 through Test No. 9

\section{APPENDIX C}

Figures C-1 through C-12... . . . . C C-3-C-14 HT-43 temperatures vs. time

Figures $\mathrm{C}-13$ through $\mathrm{C}-48$. . . . . . C-15-C-50 Thermkeep temperatures vs. axial

distance, with $\mathrm{HT}-43$

Figures C-49 through C-59 . . . . . C C-51 - C-61 T-66 temperatures vs. time

Figures C-60 through C-75 . . . . . . C-62 - C-77 Thermkeep temperatures vs. axial distance, with T-66

\section{APPENDIX D}

Figure D-1 . . . . . . . . . . . . . . D-3 Dual heat exchanger program input data

Figure D-2 . . . . . . . . . . . . . . . . D-5 Typical dual heat exchanger tubing Figure D-3 . . . . . . . . . . . . . . . D-8 Cost calculations print-out example

Figure D-4 . . . . . . . . . . . . . . . . D-9

Output data print-out example 



\section{SUMM ARY}

The objective of this work was to extend the prior work reported in NASA CR-159465, December 1978, on the development of a phase-change thermal storage system (TESS) using modified anhydrous sodium hydroxide and a single, passive, tube-intensive heat exchanger, for charging and discharging heat at approximately $584 \mathrm{~K}$ by a nonphase-change heat transfer liquid, Therminol-66.

The three parts of the present program were (1) chemical analyses on the storage medium used in the experimental model of the prior work, and examination of its heat exchanger, (2) production of additional experimental data using a different non-phase change heat transfer fluid (Caloria HT-43) to extend the validation of a computer model of the TESS, and (3) development of a new computer model of a TESS containing an additional heat exchanger for the vaporization and superheating of a power fluid for a Rankine cycle power generator, and the use of the model to develop a cost optimized reference TESS design for comparison with the single heat exchanger TESS design.

From the results, it was concluded that during the prior experimental work, no chemical degradation of the medium occurred, but some physical segregation of components was observed which had no apparent deleterious effect on the performance of the system. Physical segregation can be reversed by fully remelting the medium. No significant deterioration of the heat exchanger was found.

A computer model was successfully developed which provides for 3-way heat transfer, between each fluid and the medium, and directly between the two fluids.

The reference design of the 2-heat exchanger TESS (as compared to the single heat exchanger TESS) improved thermal performance, a lower capital cost $(\approx 58 \%)$ when credited for the cost of the external heat exchanger which it displaces, and improved overall system operating cost. 


\section{INTRODUCTION}

\section{Background}

The work reported herein is an extension of prior work under two completed contracts:

1. The development of a computer model (CM) of a phase-change thermal energy storage system (TESS) under Purchase Order No. 87-5030 from Sandia Laboratories, Livermore, California, completed in December 1976 .

2. The development of a phase-change thermal storage system using modified anhydrous sodium hydroxide for solar electric power generation, under Contract NAS3-20615 from NASA Lewis Research Center, which was reported in NASA CR-159465 in December 1978 .

This prior work included the design, construction, and testing of an experimental scale-model of a TESS suitable for use in mid-temperature range (approximately $580 \mathrm{~K})$ solar powered electricity generating systems. The model was specifically designed for reliable scaling up to meet the requirements of the Solar Total Energy Test Facility (STETF) operated by the Sandia Laboratories at Albuquerque, New Mexico.

This was used to generate experimental data with which to verify the predictions of the $\mathrm{CM}$, which was modified in several respects until acceptable agreement was obtained.

The TESS studied in the prior work consisted of a tank containing the phase-change medium, and a single "tubeintensive" heat exchanger, which is used both for charging and discharging heat by means of a non-phase-change heat transfer liquid, Therminol-66 (T-66). In the STETF, heat withdrawn from storage is delivered via the T-66 to an external heat exchanger which produces superheated toluene vapor to drive a turbine. 
The nominal composition of the phase-change thermal storage medium (Thermkeep*) is:

$\begin{array}{lc}\text { Anhydrous NaNo, Commercial Grade } & 91.8 \% \text { (wt) } \\ \mathrm{NaNO}_{3} & 8.0 \\ \mathrm{MnO}_{2} & 0.2\end{array}$

The commercial grade of $\mathrm{NaOH}$ typically contains $1-2 \%$ of $\mathrm{NaCl}$ and $1 / 2-1 \%$ of $\mathrm{Na}_{2} \mathrm{CO}_{3}$.

Phase-change TESS using Thermkeep have been under development by Comstock \& Wescott for many years in applications using electric heaters and operating at maximum temperatures as high as $725 \mathrm{~K}$ (see, for example, Ref. 1). However, the referenced work was outside the range of previous experience in three respects. First, the heat exchanger involved more and smaller tubes which required a different method of support. Second, the cycling mode of the TESS left a portion of the Thermkeep in the bottom of the tank unmelted, and because Thermkeep is a noneutectic mixture, this might lead to segregation of its components. Third, as explained in detail later, for chemical stability in contact with a steel vessel and heat exchanger, the system must have contact with air to prevent reduction of $\mathrm{NaNO}_{3}$ to $\mathrm{NaNO}_{2}$. This was provided by "breathing" during thermal cycling, and since the cycling frequency and temperature range were different from previous experience, it was not known whether the "breathing" would be adequate to maintain chemical stability.

Present Program

The present work continues the prior work by means of a three-part program:

Part I - A number of samples of Thermkeep were taken from the experimental model for chemical analysis to determine whether or not the system remained chemically stable, and whether or not segregation

* Registered Trademark Comstock \& Wescott, Inc. 
of components occurred during the prior testing program. Thereafter, the Thermkeep was melted and partially drained to permit visual examination of the upper part of the heat exchanger.

Part II - The Therminol-66 heat transfer fluid used in the prior program was replaced by Caloria HT43, and an additional set of experimental data obtained for further verification of the computer model with a fluid of different physical properties.

Part III - An earlier study had suggested that TESS and overall system benefits might be obtained in the STETF if a second heat exchanger were incorporated in the TESS in which the toluene vapor for the turbine would be generated, thus eliminating the external toluene boiler. Therefore, a new computer model was developed to predict the performance of such a TESS. This computer model was used to produce a reference design, including construction cost, of a unit suitable for installation in the Sandia STETF. This was compared with the design of the single heat exchanger unit produced in the prior work. 



\author{
PART I \\ ANALYSIS OF THERMKEEP SAMPLES \\ AND EXAMINATEON OF HEAT EXCHANGER
}

The experimental model produced under Contract NAS3-20615 remained in the state in which it was left after several weeks of thermal cycling, as described in NASA CR-159465. During this cycling the thermal gradient causes the Thermkeep in the upper section of the vessel to be predominantly in the liquid state, the Thermkeep at the bottom is predominantly solid, with an intermediate condition between. Since Thermkeep is a non-eutectic composition, segregation of components may have occurred. In addition, some $\mathrm{NaNO}_{3}$ may have been reduced to $\mathrm{NaNO}_{2}$ by the mechanism described below. In order to clarify these points, a series of samples of Thermkeep were taken for chemical analysis.

Chemical Analysis of Thermkeep Samples

The thermal insulation and the external heating shroud (used to control the thermal gradient in the insulation) were removed from one side of the vessel. With the vessel at room temperature, seven $10.8 \mathrm{~cm}$ diameter holes were cut along a vertical line on the vessel wall by means of a hole saw. The centers of the holes are 5.1 , $32.7,60.7,88.0,116.6,135.5,170.8$ centimeters from the bottom of the vessel. These locations span the total height of the solid Thermkeep.

The removal of these sections exposed the solid Thermkeep. Approximately $0.23 \mathrm{~kg}$ was removed in the form of chips through each hole, after which the holes were temporarily covered to prevent absorption of atmospheric moisture and $\mathrm{CO}_{2} \cdot$

During the removal of the sample from the third hole from the bottom, a very small penetration accidentally occurred in one of the heat exchanger coils. However, this was in an accessible location and was repaired. This was done by flowing nitrogen through the tube while it was being welded from the outside. This prevented obstruction of the tube bore at the weld. The sampling openings were then sealed by steel patches welded onto the tank wall. 
Examination of Heat Exchanger

The exposed section of the tank was covered by temporary insulation. The heating system which is used for charging the TES unit was activated and the Thermkeep was completely melted. Approximately $340 \mathrm{~kg}$ of Thermkeep were withdrawn from the tank into a steel drum which lowered the level sufficiently so that the upper third of the heat exchanger was uncovered. The Thermkeep in the drum was covered to prevent absorption of moisture and $\mathrm{CO}_{2}$ and allowed to self-cool.

The Thermkeep remaining in the TES unit was allowed to solidify and cool. The cover of the vessel was removed and the interior of the exposed interior surfaces were examined. The heat exchanger was photographed to record its appearance. Its condition was compared to photographs made of the heat exchanger at the time of manufacture and assembly in the tank. Physically the heat exchanger appeared to have undergone no significant distortion. The supporting structure appeared to be in good condition and was adequate for the planned experimental work. The metal surfaces which were exposed to air above the highest level of the liquid Thermkeep were found to have acquired a light coating of sodium carbonate crystals. This condition has been found consistently in Thermkeep systems on those surfaces which are exposed to ambient air drawn into the clearance space by cycling. This material was easily brushed off and the underlying steel showed no abnormal signs of corrosion.

As a result of the examination it was concluded that no internal repair or maintenance was required before continuing with the program.

Heat Transfer Fluid

The original program plan was to use Silicone $B$ as the heat transfer fluid for the test program. This fluid is produced by Dow-Corning and has been designated by three different names: Silicone B, Siltherm 800, and $\mathrm{X} 2-1162$. A quantity of this material was obtained, and a qualitative test made to determine whether any obvious interaction occurred when Thermkeep and Silicone $B$ are in contact at a temperature of $589 \mathrm{~K}(600 \mathrm{~F})$. It was found that when a small quantity of either Thermkeep or 
reagent grade $\mathrm{NaOH}$ is dropped into the $589 \mathrm{~K}(600 \mathrm{~F}$ ) fluid, a vigorous evolution of gas occurs, and the temperature of the liquid drops promptly. Analysis of the tests indicated that decomposition of the fluid was initiated by the $\mathrm{NaOH}$. The decomposition products are lower molecular weight cyclic molecules such as tetramethyl cyclosiloxane, which are more volatile than the Silicone B. These compounds are reported to be non-toxic.

In view of this instability of the Thermkeep-Silicone $B$ system, the NASA Project Manager directed that a substitute heat transfer fluid which is stable in the presence of Thermkeep at $589 \mathrm{~K}(600 \mathrm{~F})$ should be identified and substituted, since it is an objective of the program not only to verify the computer model of the system with a second heat transfer fluid, but also to test materials which can be used in a practical system. It was subsequently decided that Caloria HT-43 (a product of Exxon Co.) should be used in the testing program.

Chemical Analysis of Thermkeep

The samples of Thermkeep were analyzed by Skinner \& Sherman, Inc. for percentage by weight of $\mathrm{NaOH}, \mathrm{NaNO}_{3}$, $\mathrm{NaNO}_{2}, \mathrm{MnO}_{2}, \mathrm{Na}_{2} \mathrm{CO}_{3}$, and $\mathrm{NaCl}$. A copy of Skinner \& Sherman's report is included herewith as Appendix A. The values reported are repeated in Table I which also shows the percentage of $\mathrm{NaOH}$ and the total of the six constituents for each sample.

Samples 1 and 2 were taken from a previously unopened drum of Thermkeep flakes and represent the batch of material from which the TES unit was originally charged. The remaining samples are the pairs which were taken from the seven openings in the side of the TES unit.

All constituents except $\mathrm{NaNO}_{2}$ were independently determined. $\mathrm{NaNO}_{2}$ was obtained as the difference between a determination of total nitrogen (not shown in the report), and the $\mathrm{NaNO}_{3}$. The reported results are thus based on six independent determinations. 
ANALYSIS OF THERMKEEP FROM TES VESSEL, AND ORIGINAL MATERIAL (\% by weight)

\begin{tabular}{|c|c|c|c|c|c|c|c|c|c|}
\hline $\begin{array}{c}\text { Sample } \\
\text { No. }\end{array}$ & $\begin{array}{c}\text { Location } \\
\text { No. } \\
\end{array}$ & $\begin{array}{l}\text { Dist. } \\
\text { from } \\
\text { bottom, } \\
\text { cm } \\
\end{array}$ & $\underline{\mathrm{NaOH}}$ & $\mathrm{NaNO}_{3}$ & $\mathrm{NaNO}_{2}$ & $\underline{\mathrm{MnO}_{2}}$ & $\mathrm{Na}_{2} \mathrm{CO}_{3}$ & $\underline{\mathrm{NaCl}}$ & Total \\
\hline 1 & \multirow{2}{*}{ Orı. Mat'I } & \multirow{2}{*}{ N/A } & 86.94 & 8.467 & .0306 & .172 & 2.62 & 2.03 & 100.26 \\
\hline 2 & & & 88.74 & 7.477 & .0307 & .171 & 1.45 & 1.98 & 99.85 \\
\hline 3 & \multirow{2}{*}{1} & \multirow{2}{*}{172} & 91.50 & 4.652 & .0020 & .101 & 2.85 & 1.73 & 100.84 \\
\hline 4 & & & 91.55 & 5.278 & .0021 & .085 & 1.48 & 1.35 & 99.75 \\
\hline 5 & \multirow{2}{*}{2} & \multirow{2}{*}{144} & 91.17 & 5.041 & .0023 & .113 & 1.55 & 1.47 & 99.35 \\
\hline 6 & & & 91.74 & 5.326 & .0022 & .210 & 1.43 & 1.63 & 100.24 \\
\hline 7 & \multirow{2}{*}{3} & \multirow{2}{*}{$\operatorname{Il7}$} & 91.88 & 4.503 & .0019 & .083 & 1.38 & 1.68 & 99.53 \\
\hline 8 & & & 92.23 & 4.377 & .0023 & .110 & 1.35 & 1.85 & 99.92 \\
\hline 9 & \multirow[t]{2}{*}{4} & \multirow{2}{*}{89} & 89.50 & 5.623 & .0023 & .102 & 2.89 & 1.65 & 99.77 \\
\hline 10 & & & 91.66 & 4.878 & .0026 & .095 & 1.36 & 1.76 & 99.76 \\
\hline 11 & \multirow{2}{*}{5} & \multirow{2}{*}{62} & 94.30 & 2.791 & .0017 & .042 & 1.44 & 1.53 & 100.10 \\
\hline 12 & & & --- & --- & --- & -- & --- & --- & --- \\
\hline 13 & \multirow{2}{*}{6} & \multirow{2}{*}{34} & 93.93 & 2.707 & .0021 & .057 & 1.40 & 1.76 & 99.86 \\
\hline 14 & & & 94.59 & 2.546 & .0032 & .053 & 1.32 & 1.25 & 99.76 \\
\hline 15 & \multirow{2}{*}{7} & \multirow{2}{*}{6} & 89.17 & 5.772 & .0098 & .137 & 2.88 & 1.58 & 99.55 \\
\hline 16 & & & 89.88 & 5.138 & .0095 & .172 & 2.90 & 1.43 & 99.53 \\
\hline
\end{tabular}


The fact that in every case the deviation of the total of the six from $100 \%$ was no greater than $0.65 \%$ was an indication of the precision of the analytical work. These totals also indicated that the samples absorbed only an insignificant amount of water from the ambient air during the sampling procedure.

As previously described, the samples were removed from the TES unit by cutting $10.8 \mathrm{~cm}$ diameter circular sections from the tank wall. The samples were removed within a distance of about $5.1 \mathrm{~cm}$ from the tank wall. The samples were not mixed, so that the first sample removed may represent the material closest to the tank wall, and the second sample the material taken from the deeper layer.

Prior to removing the samples, the TES unit was last operated in the program described in Report CR-159465. The final tests were simulations of the solar daily cycle, the results of which are shown in Figs. 91-100 of that report.

\section{Discussion of Analytical Results}

A significant result was that the total sodium nitrate content of the samples taken from the TES unit was significantly lower than that in the original material.

Figures 1, 2, and 3 show the averages of the two samples taken from each opening in the tank, plotted against the location of the opening. The distance between the centers of adjacent openings was $27.6 \mathrm{~cm}$.

The curves for sodium nitrate, sodium carbonate, manganese dioxide, and sodium nitrite have similar shapes in which the percentage of these constituents tends to be lowest at locations 5 and 6 and highest at location 7 (nearest the bottom of the vessel). This suggests that in a zone about $5.1 \mathrm{~cm}$ thick adjacent to the wall of the vessel, there was some vertical segregation of these components of Thermkeep. 


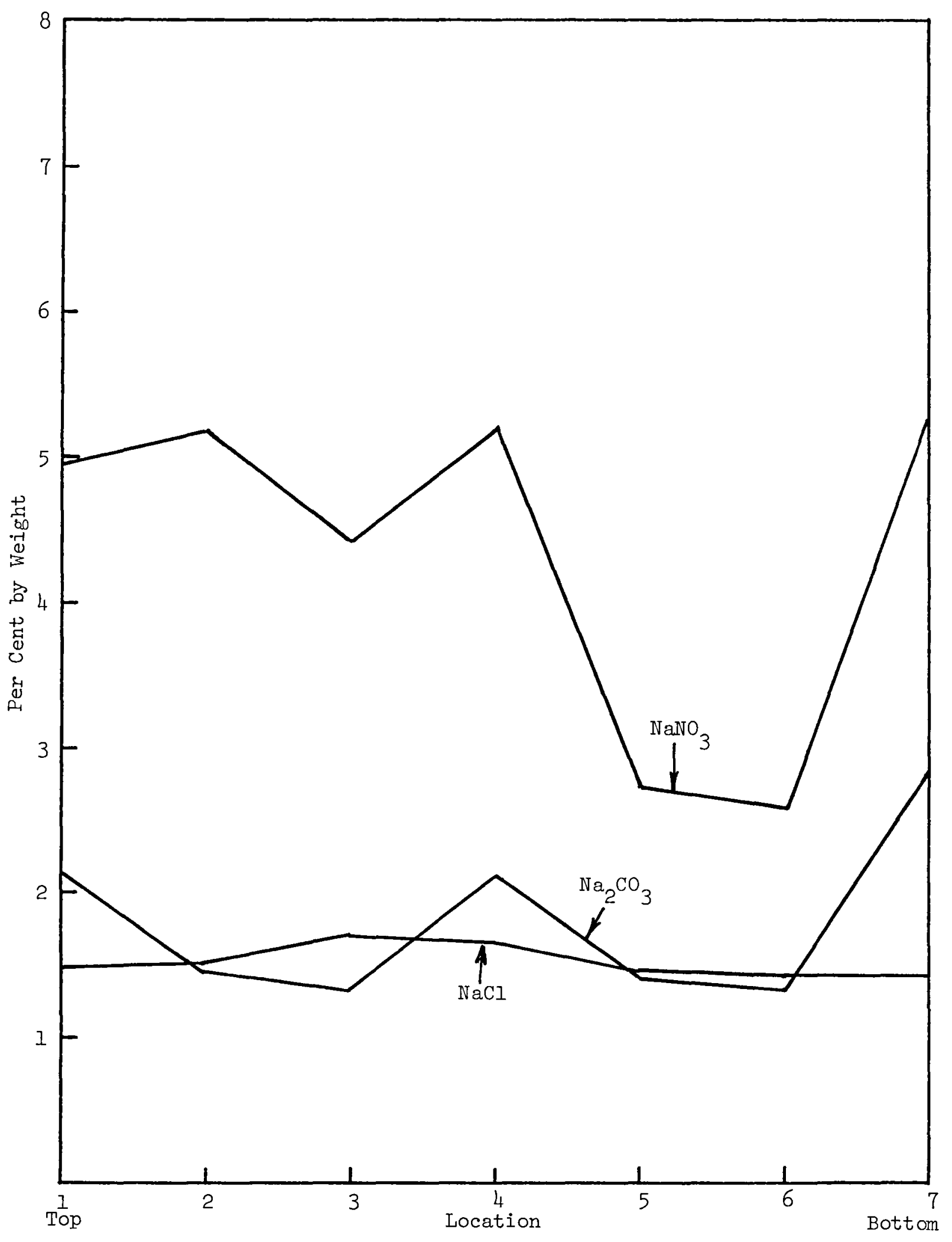

Figure 1. Variation of $\mathrm{NaNO}_{3}, \mathrm{Na}_{2} \mathrm{CO}_{3}$ and $\mathrm{NaCl}$ with vertical locatıon in tank. 


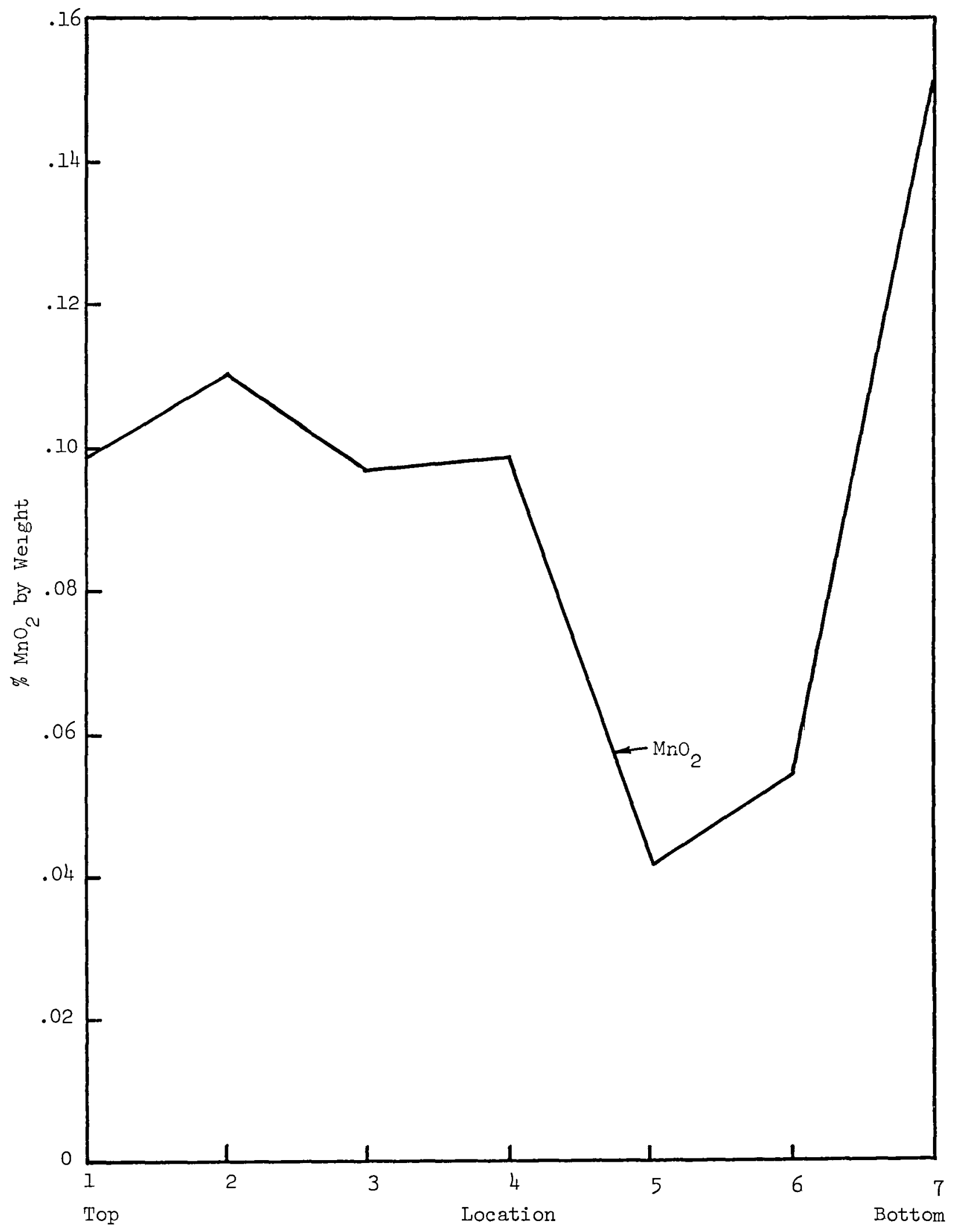

Flgure 2. Varlation of $\mathrm{MnO}_{2}$ with vertical location in tank. 


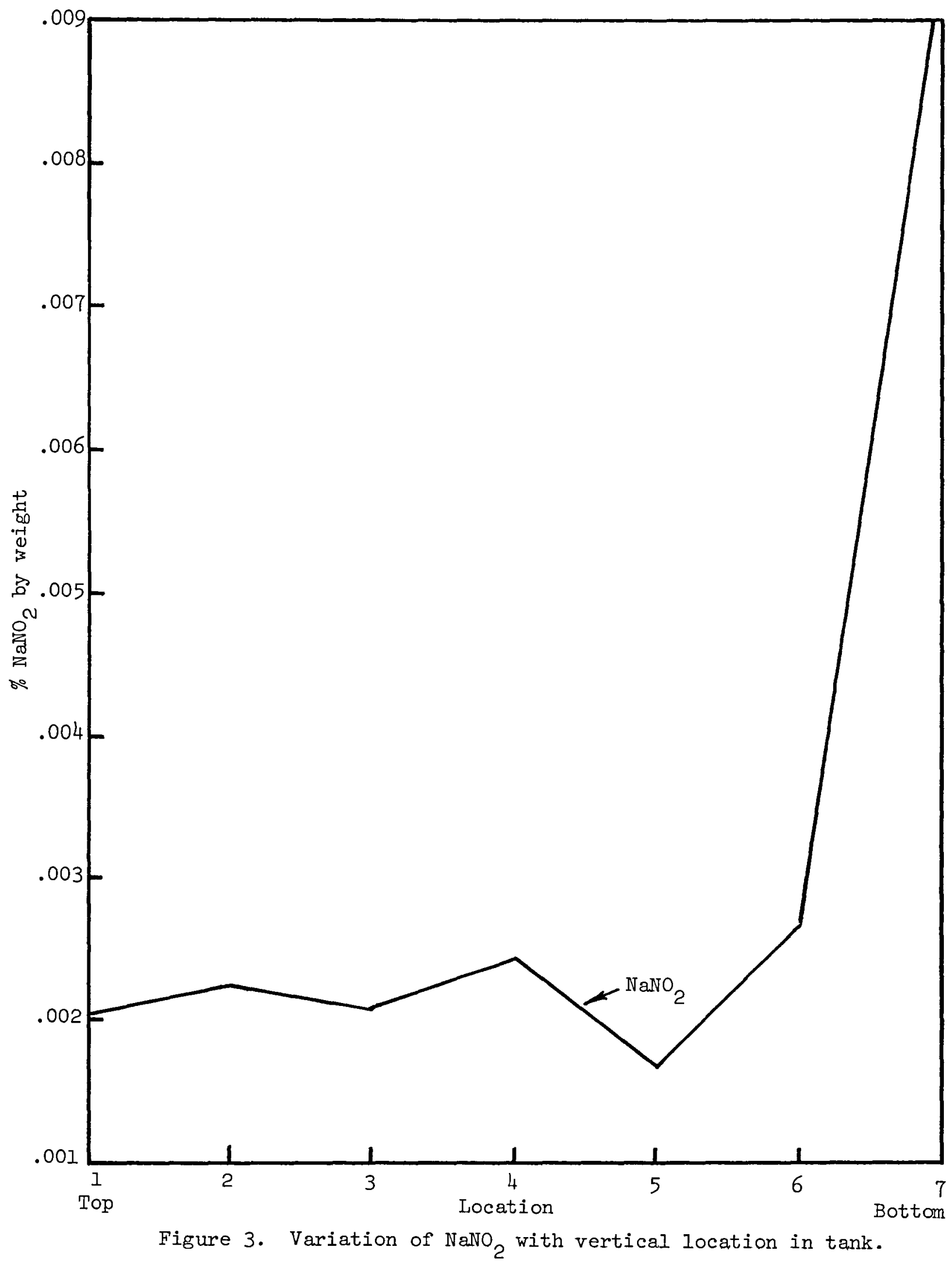


In Figure 4 the ratio of the average $\mathrm{NaOH}$ content to the average $\mathrm{NaNO}_{3}$ content, for each pair of samples, is plotted against the sampling location, showing that this ratio for all samples is significantly higher than that of the original Thermkeep, and that at locations 5 and 6 , it is higher than at the other locations.

Figure 5 is a phase diagram (Ref. 2) for the system $\mathrm{NaOH}-\mathrm{NaNO}_{3}$ which shows that the solid which forms upon cooling was higher in sodium hydroxide than was the liquid phase from which it was deposited. Although this phase diagram does not truly represent Thermkeep because of presence of $2-3 \%$ of $\mathrm{NaCl}$ and $\mathrm{Na}_{2} \mathrm{CO}_{3}$, it is probable that during solidification a local variation in composition in the vicinity of the heat exchanger coil would result. The solid close to the tubes would be expected to be lower in sodium nitrate than the solid near the centerline of the heat exchanger coils or in the spaces between the coils. This might also account for the low nitrate content of the Thermkeep which solidified on the vessel walls as a result of heat lost through the insulation.

\section{Chemistry of Thermkeep-Steel System}

Studies prior to this contract have revealed some of the chemical characteristics of the Thermkeep-steel system at $756 \mathrm{~K}(900 \mathrm{~F})$. Chemical stability requires contact with air. This is provided by the "breathing" of ambient air in and out of the clearance space above the Thermkeep during thermal cycling due to the changing volume of the Thermkeep. The reactions which are believed to take place are:

$$
\begin{aligned}
& 2 \mathrm{Fe}+6 \mathrm{NaOH} \rightarrow \mathrm{Na}_{2} \mathrm{Fe}_{2} \mathrm{O}_{4}+3 \mathrm{H}_{2}+2 \mathrm{Na}_{2} \mathrm{O} \\
& x \mathrm{Fe}+\mathrm{y} \mathrm{NaNO}_{3} \rightarrow \mathrm{Fe}_{x} \mathrm{O}+\mathrm{y} \mathrm{NaNO}_{2} \\
& \mathrm{y} \mathrm{NaNO}_{2}+\frac{\mathrm{y}}{2} \mathrm{O}_{2} \stackrel{\left(\mathrm{MnO}_{2}\right)}{\longrightarrow} \mathrm{y} \mathrm{NaNO}_{3}
\end{aligned}
$$


The net reaction is:

$$
\begin{array}{r}
(x+2) \mathrm{Fe}+2 \mathrm{NaOH}+\frac{\mathrm{y}}{2} \mathrm{O}_{2}+2 \mathrm{H}_{2} \mathrm{O} \frac{\left(\mathrm{MnO}_{2}\right)}{2}> \\
\mathrm{Na}_{2} \mathrm{Fe}_{2} \mathrm{O}_{4}+\mathrm{Fe}_{\mathrm{x}} \mathrm{O}_{\mathrm{y}}+3 \mathrm{H}_{2}
\end{array}
$$

Eq. I) is the "corrosion reaction." $\mathrm{Na}_{2} \mathrm{Fe}_{2} \mathrm{O}_{4}$ is the principal corrosion product, and has been identified by $\mathrm{X}$-ray diffraction tests. The presence of hydrogen has also been confirmed. Corrosion rates in inches per year (ipy) of penetration at $756 \mathrm{~K}(900 \mathrm{~F})$ have been measured at less than $2.5 \times 10^{-3} \mathrm{~cm} /$ year $(0.001 \mathrm{ipy})$. In the present application at about $584 \mathrm{~K}(591 \mathrm{~F})$, corrosion is expected to be insignificant in the time of operation, and no measurements were undertaken.

Eq. 2) represents the formation of iron oxide of unidentified composition, by reduction of $\mathrm{NaNO}_{3}$. Salt baths similar in composition to Thermkeep are widely used for metal cleaning, at temperatures of 756-367 K (900-1100 F). These are known to produce oxide coatings on the metal by $\mathrm{NaNO}_{3}$ which is reduced to $\mathrm{NaNO}_{2}$.

Eq. 3) represents the oxidation of $\mathrm{NaNO}_{2}$ to $\mathrm{NaNO}_{3}$ by oxygen from the air. This reaction is catalyzed by $\mathrm{MnO}_{2}$.

$\mathrm{Eq}$. 4) represents the reaction of the $\mathrm{Na}_{2} \mathrm{O}$ formed by $\mathrm{Eq}$. I) with moisture introduced by the "breathing" of humid air. 


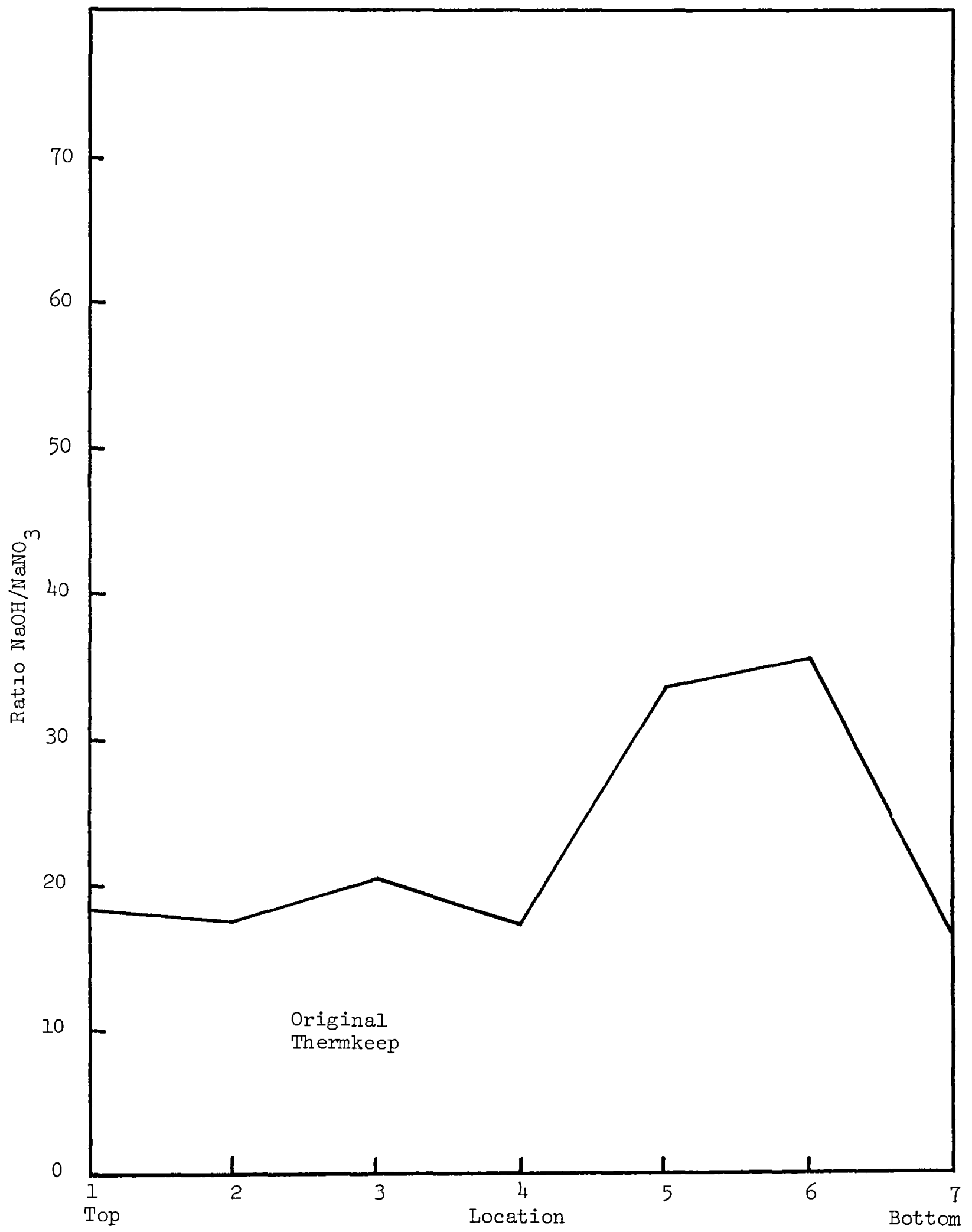

Figure 4. Variatıon of $\mathrm{NaOH} / \mathrm{NaNO}_{3}$ ratio with vertical location in tank. 


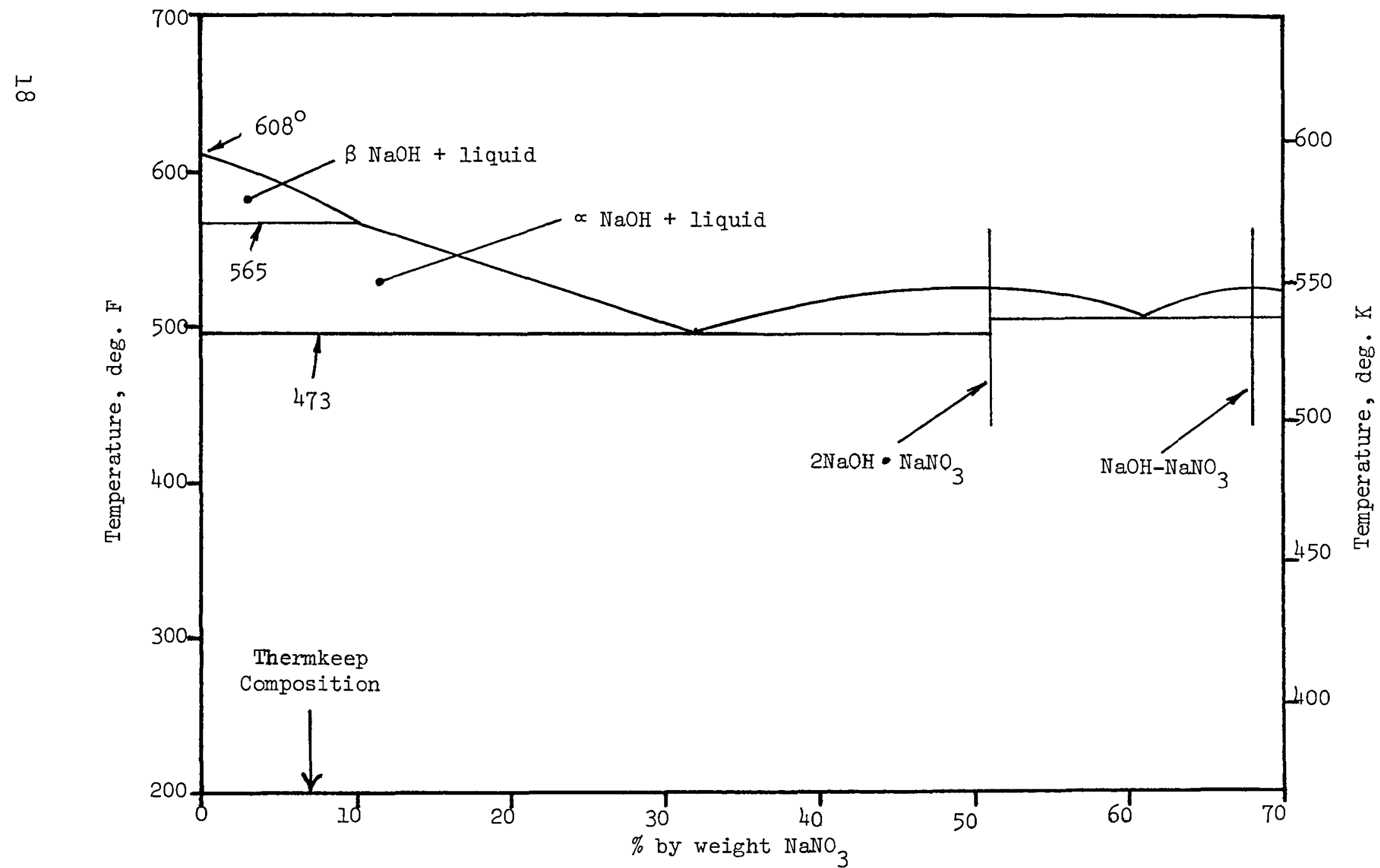

Figure 5. Phase Diagram, NaOH-NaNO 3 
One purpose of the chemical analyses was to determine whether sufficient oxygen was absorbed by the system to maintain the original level of $\mathrm{NaNO}_{3}$. Since the analysis showed that the $\mathrm{NaNO}_{2}$ in the system was an order of magnitude lower than in the original Thermkeep, it was concluded that the oxygen absorption rate was sufficient to prevent significant conversion of $\mathrm{NaNO}_{3}$ to $\mathrm{NaNO}_{2}$.

The analyses showed that the $\mathrm{NaNO}_{3}$ content of the samples was substantially lower than that of the original Thermkeep. This raises the question of whether or not nitrogen may have been lost from the system. Based upon prior work, it is believed that loss of nitrogen cannot account for this phenomenon.

Prior experiments have been conducted on the Thermkeepiron system, at $756 \mathrm{~K}(900 \mathrm{~F})$, in steel containers which were hermetically sealed to prevent entrance of air, in which the pressure was monitored. It was observed that such a system, at $756 \mathrm{~K}$, initially develops a subatmospheric pressure which remains stable for about five days, after which it rises abruptly. The rise in pressure is found to be due to the generation of ammonia. This behavior is due to the sequential reduction of $\mathrm{NaNO}_{3}$-first to $\mathrm{NaNO}_{2}$, then to $\mathrm{NH}_{3}$, according to the following reactions:

$$
\begin{gathered}
8 \mathrm{Fe}+24 \mathrm{NaOH} \rightarrow 4 \mathrm{Na}_{2} \mathrm{Fe}_{2} \mathrm{O}_{4}+12 \mathrm{H}_{2}+8 \mathrm{Na}_{2} \mathrm{O} \\
3 \mathrm{NaNO}_{3}+3 \mathrm{H}_{2} \rightarrow 3 \mathrm{NaNO}_{2}+3 \mathrm{H}_{2} \mathrm{O} \\
3 \mathrm{NaNO}_{2}+9 \mathrm{H}_{2} \rightarrow 3 \mathrm{NH}_{3}+3 \mathrm{H}_{2} \mathrm{O}+3 \mathrm{NaOH} \\
6 \mathrm{Na}_{2} \mathrm{O}+6 \mathrm{H}_{2} \mathrm{O} \rightarrow 12 \mathrm{NaOH}
\end{gathered}
$$


Summing these equations gives:

$$
\begin{aligned}
8 \mathrm{Fe}+9 \mathrm{NaOH}+ & 3 \mathrm{NaNO}_{3} \rightarrow \\
& 4 \mathrm{Na}_{2} \mathrm{Fe}_{2} \mathrm{O}_{4}+3 \mathrm{NH}_{3}+2 \mathrm{Na}_{2} \mathrm{O}
\end{aligned}
$$

Based upon this chemistry, and the fact that no $\mathrm{NH}_{3}$ has ever been detected in the clearance space of a cycling system which has had unobstructed access to air, it was concluded that nitrogen had not been lost.

The fact that the $\mathrm{NaNO}_{3}$ content of all samples was substantially lower than that of the original Thermkeep charged into the TES tank has been attributed to segregations of chemical components; a small degree of segregation in the vertical direction and a larger amount in the radial direction.

The radial segregation was believed to be explainable on the basis of the $\mathrm{NaOH}-\mathrm{NaNO}_{3}$ phase diagram, Figure 5 , which shows that during the thermal discharge cycles the solid which forms on the surfaces of the tubular heat exchanger is richer in $\mathrm{NaOH}$ than the liquid from which it crystallizes, or equivalently the solid has a lower percentage of $\mathrm{NaNO}_{3}$ than the liquid. The samples used for analysis were removed through openings in the tank wall and are therefore solid which formed on or close to the wall. Prior to removal of the samples, the last operation of the TES unit (Fig. 100, Report NASA CR-159465) consisted of a thermal discharge which produced a solid layer on the heat exchanger surfaces, leaving liquid in the centers of the tubular helices and near the tank wall.

Upon termination of the test the Thermkeep temperatures in the upper two-thirds of the tank were above $565 \mathrm{~K}$, which as shown by the phase diagram would contain $50 \%$ or more of Iiquid. 
After completion of the test the circulation of the heat transfer liquid was stopped and the TES unit allowed to self-cool by losing heat through the insulation. This would cause the remaining liquid to solidify starting at the tank walls. The solid forming at and near the walls would be low in $\mathrm{NaNO}_{3}$, while the remaining liquid would increase in $\mathrm{NaNO}_{3}$ until the eutectic composition was reached at some distance from the walls. This explanation is, of course, over simplified because it does not take account of the $\mathrm{Na}_{2} \mathrm{CO}_{3}$ and $\mathrm{NaCl}$ which total approximately $4 \%$ of the original Thermkeep composition.

A number of tests were considered which might verify the presumption that no nitrogen has been lost, and shed more light on the segregations. However, the opportunity to obtain more information on the system in its original state was lost when the Thermkeep was completely melted for draining and examination of the heat exchanger.

\section{Summary of Chemical Analyses of Thermkeep}

The following conclusions were drawn as a result of the observations described above:

1. The accuracy of the analyses was good.

2. There were no significant compounds present in the system other than those analyzed.

3. The samples were not significantly contaminated with $\mathrm{H}_{2} \mathrm{O}$.

4. No significant $\mathrm{NaNO}_{2}$ was present and, therefore, 1) no nitrogen was lost from the system, and 2) air "breathing" is adequate to maintain chemical stability.

5. Variations in the $\mathrm{NaOH} / \mathrm{NaNO}_{3}$ ratio were a result of segregation of components during cooling. 
6. A small vertical segregation existed as shown by the high $\mathrm{NaOH} / \mathrm{NaNO}_{3}$ ratio at locations 5 and 6 .

7. Since the $\mathrm{NaOH} / \mathrm{NaNO}_{3}$ ratio at all seven sampling locations was less than that of the original material, there must also be a radial segregation from the tank wall inward. These radial segregations had no observed deleterious effect on the operation of the TES system in prior tests, and are probably acceptable if the system had arrived at a stable state as a result of the cycling carried out in the prior program of testing. To determine whether stabilization was achieved will require further testing.

8. If further cycling produces progressive segregation to the point where the performance of the system is unacceptably degraded, this condition could be reversed by periodically over-charging the TES unit and allowing the liquefield Thermkeep to become homogeneous by natural circulation of the liquid. 
PART II

VERIFICATION OF COMPUTER MODEL

BY EXPERIMENTAI DATA

Introduction

The purpose of this work was to extend the experimental verification of the computer model (CM) of the single heat exchanger TESS, by means of additional data obtained from the experimental scale-model. The new data were obtained through the substitution of a different heat transfer fluid for the Therminol-66 (T-66) used previously. The substitute fluid was to have physical properties as different from those of T-66 as possible, in order to demonstrate that the CM could be used with confidence to predict TESS performance for a range of fluids.

The program of testing was to duplicate the prior program, so far as heat transfer rates were concerned, by adjusting flow rates to compensate for the different physical properties of the new fluid. The fluid tentatively selected was Silicone B (a product of Dow-Corning), because its physical properties are quite different from those of T-66, and it has superior thermal stability, although its cost is substantially higher. When, as described in Part $I$, it was found to be reactive with molten Thermkeep, it was rejected on the basis of safety, and Caloria HT-43 was substituted.

Heat Transfer Fluid

Caloria $\mathrm{HT}-43$ has a higher specific heat ( $\mathrm{Cp}$ ) but a lower density $(\rho)$ than $T-66$, and flow rates are inversely proportional to the product of these values. The following compares the two fluids at $316 \mathrm{C}(600 \mathrm{~F})$ :

$\begin{array}{rccc} & \rho & \mathrm{Cp} & \\ & \underline{\mathrm{g} / \mathrm{cm}^{3}} & \mathrm{~J} /(\mathrm{g} \cdot \mathrm{K})_{-} & \underline{\rho \mathrm{Cp}} \\ \mathrm{T}-66 & .805 & 2.63 & 2.12 \\ \mathrm{HT}-43 & .649 & 2.93 & 1.90\end{array}$


Since $\rho C p$ for HT-43 is $90 \%$ that of T-66, the flow rates necessary to effect the same heat rates at $589 \mathrm{~K}$ fluid temperatures must be increased by about 10\%. The highest steady state flow rate in the test plan was 0.35 $\mathrm{kg} / \mathrm{sec}(6.6 \mathrm{gpm})$ for T-66, while the pump is capable of $0.40 \mathrm{~kg} / \mathrm{sec}(7.6 \mathrm{gpm})$. The only test in which the heat rates were not duplicated was the solar cycle, where during charging the HT-43 will reach the maximum pump rate sooner than did the T-66. Since the actual flow rate is a computer input, this was simulated in the computer program.

\section{Experimental Model}

The experimental model is described in detail in NASA CR-159465. Briefly, it consists of a steel cylindrical thermal storage tank $2.23 \mathrm{~m}$ in height and $0.70 \mathrm{~m}$ in diameter containing $1500 \mathrm{~kg}$ of Thermkeep. The heat exchanger consists of 25 helical steel tubes, $0.635 \mathrm{~cm}$ outside diameter and $23.1 \mathrm{~m}$ long, which are manifolded at the top and bottom of the tank for parallel flow of the heat transfer fluid, downward during charging, and upward during discharging. Temperatures are measured by 20 thermocouples in wells extending through the side of the tank into the Thermkeep to two different depths, and additional couples on the outside of the tank.

The temperature of the fluid entering and leaving the tank and at other points in the system, as well as other system temperatures, are also measured by thermocouples.

The tank is covered by an inner layer of thermal insulation $0.457 \mathrm{~m}$ thick, and an inner steel shroud carrying a grid of electric heating elements. This is covered by an additional layer of insulation $0.152 \mathrm{~m}$ thick and an outer steel shroud. The heated shroud is used to establish a desired thermal gradient in the inner insulation in order to simulate insulation heat loss which would occur in a larger unit.

The auxiliary equipment provides for the supply of heat transfer fluid to the TESS at predetermined flow rates and temperatures for charging and discharging, at constant flow rates and at programmed variable rates to permit simulation of solar cycles. 
The experimental model has approximately one-tenth the capacity of a TESS suitable for use in the Sandia Albuquerque Solar Total Energy Test Facility. The height of the model and consequently the length of each heat exchanger element are of full scale; the crosssection and consequently the number of heat exchanger elements are one-tenth of full scale.

Preparation of Test Unit

After samples were taken from the storage unit, about $430 \mathrm{~kg}$ (950 lbs) of Thermkeep flakes were added to replace what had been drained to allow visual inspection of the heat exchanger. During previous testing the liquid level of Thermkeep was about $15 \mathrm{~cm}$ lower than necessary to cover the entire heat exchanger at the maximum temperature, since a large portion of Thermkeep usually remains solid at the bottom during normal duty cycles. Therefore, enough Thermkeep was added to increase the average level by $15 \mathrm{~cm}$.

The storage tank was closed and covered with insulation. The heated shroud was then replaced and its heating elements checked and reconnected. The purpose of the heated shroud was to produce an elevated "ambient" temperature for the tank. The shroud was turned on and over a period of ten days was balanced so that the top, sides, and bottom were at an average temperature of about $493 \mathrm{~K}$.

All of the original heat transfer fluid (T-66) was drained from the system and air was blown through the pipes and coils for 24 hours. The system was then charged with 10 gallons of Caloria HT-43 (HT-43) which was pumped through all of the plumbing, collected, and circulated once again. Then the HT-43 was drained and air was pumped through the pipes for 24 hours. The procedure was repeated with fresh HT-43. After draining the T-66 and after each HT-43 flushing, the waterimmersed cooling coil was removed, inverted, and drained. This coil was the only location where more than one pint of liquid could collect. The liquid content of the heat exchanger was measured between flushings by balancing its pressure head against a water manometer, on the upper and lower manifolds, and found to be no more than $250 \mathrm{~cm}^{3}$. Therefore, in the worst case, each 38 liter ( $10 \mathrm{gal}$ ) flush was at least a 100 to 1 
dilution, and with the final charge of 265 liters (70 gal), another 1000 to 1 dilution.

The unit was heated to various states of charge and the liquid level of Thermkeep was measured with a dipstick. This showed that the unit contained $1515 \mathrm{~kg}$ of Thermkeep, or about $75 \mathrm{~kg}$ ( 165 las) more than before disassembly, and that none of the heat exchanger surface was exposed above the Thermkeep level during normal cycling.

Two minor problems were encountered before the start of tests: The cooling water barrel had several leaks and was replaced, and thermal siphoning to the expansion tank became an increasing problem. The siphon pathway was traced to a broken relief valve between the heating reservoir and the expansion tank, and the valve was removed. An alternate escape pathway already exists with a hand-operated valve, and this was fixed in the open position.

All thermocouples were found to be in working order and were connected to a 60-channel "Datalogger" (FLUKE Model 2240B), which automatically records temperatures on paper tape, on a predetermined schedule.

Testing Procedure

The experimental data taken include the flow rate of the heat transfer fluid, $\mathrm{HT}-43$, its temperature entering and leaving the TES unit, and temperatures of the Thermkeep in the TES unit measured by sets of thermocouples in wells extending into the tank, in two vertical rows, one to a depth of $11.0 \mathrm{~cm}$ and the other to a depth of 27.3 $\mathrm{cm}$ from the tank wall (the tank diameter is $69.9 \mathrm{~cm}$ inside).

The temperature data, recorded on paper tape by the Datalogger, were manually transferred to files of a small computer. The computer model (CM) of the TESS required a larger computer located in an outside facility for the CM performance predictions. The results of these predictions were also transferred to the small computer file, which was then used to print graphical displays of the experimental data and superposed CM predictions. In these graphs, the maximum deviation of the printed point from the true point is $2.5^{\circ} \mathrm{C}$. 
Inputs into the CM program include the physical properties of the heat transfer fluid, and its flow rate. Twenty-five elements are used in the analysis. The initial condition data for the CM includes the temperature of the Thermkeep in each element, as measured at the start of the corresponding test.

The experimental data and the CM predictions of performance of Thermkeep temperatures vs. time are shown in several sets of computer-printed graphs which are to be found in Appendices $B$ and $C$. Temperat ures were recorded and graphed at half-hour intervals, but the data in Appendices $B$ and $C$ are abbreviated to conserve space. Superposed on the data are the temperatures predicted by the computer model. The graphs show the Thermkeep temperatures vs. the location in the tank, measured from the top of the tank. Also shown is the location of the upper surface of the Thermkeep as calculated by the CM; this location depends upon the temperature variation within the tank and the fraction of Thermkeep which is solid.

\section{Enthalpy-Temperature of Thermkeep}

The CM uses a table relating the enthalpy and temperature of the Thermkeep, which is used to determine the energy of each of the finite elements. Data for the table were to have been determined under a subcontract of the preceding contract. This failed to produce useful information and in its place data obtained by Comstock \& Wescott during large scale cooling tests were used. These data were input to the $\mathrm{CM}$ in the form of a table. The table used in the prior work to correlate data and CM predictions is identified as "Table 4" and is shown graphically in Figure 6 .

The ability to produce large numbers of graphical displays of superposed experimental data and $\mathrm{CM}$ predictions quickly and inexpensively by computer data processing enabled a more complete analysis of the temperatureenthalpy relation than was possible during the preceding study. This showed that Table 4 is a less than ideal temperature-enthalpy representation, and also that some degree of hysteresis occurs in the latent heat range during heating-cooling cycles. In order to study these effects, the enthalpy table was adjusted by trial-anderror until two new tables were produced, one which 


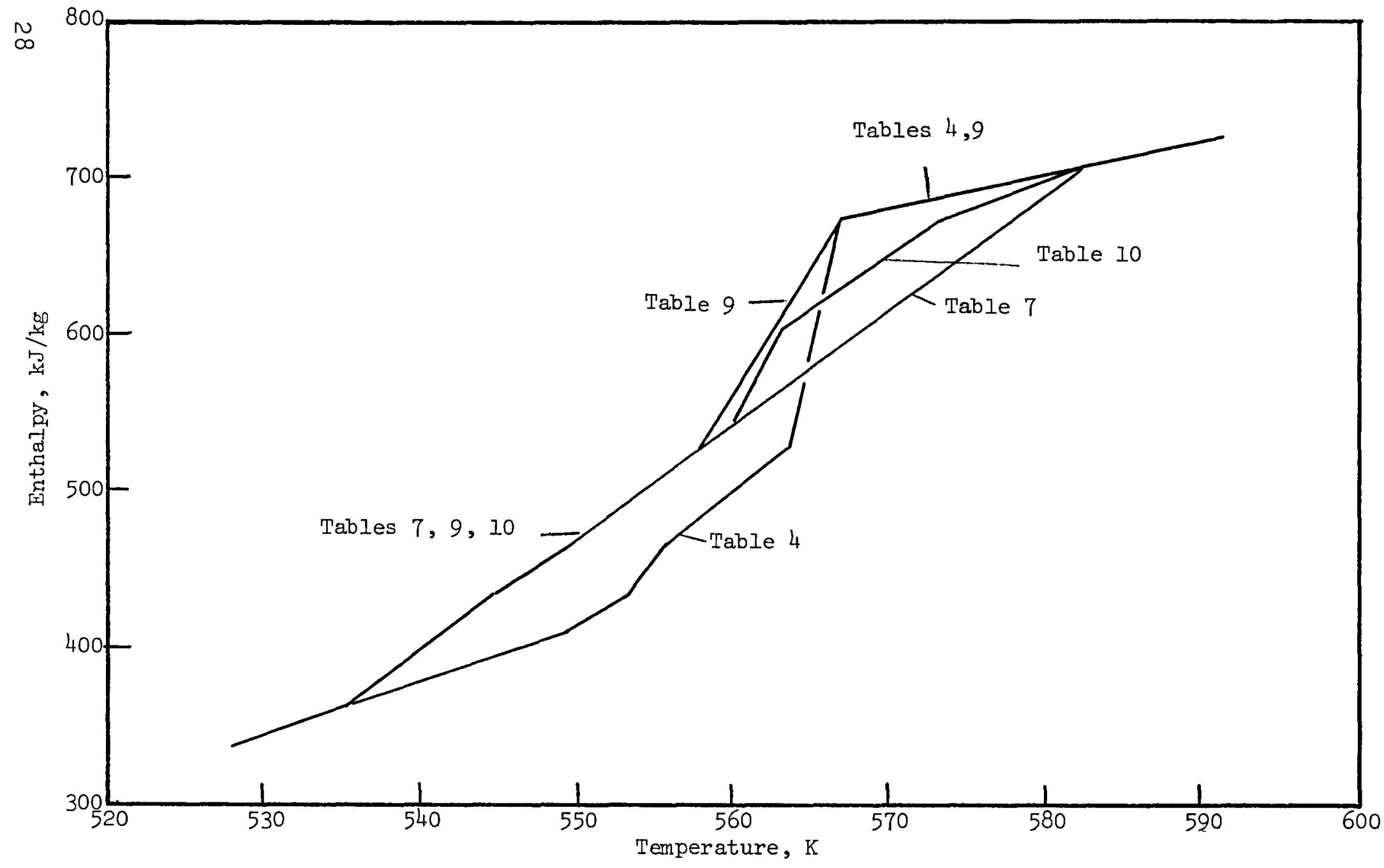

Figure 6. Temperature-Enthalpy Tables for Thermkeep 
correlates the CM predictions better with cooling data, and another with heating data. These are shown graphically in Figure 6, where Table 7 is used for heating, and Table 9 for cooling.

In addition, Table 10 was constructed and used as a compromise to correlate both heating and cooling data.

\section{Hysteresis Study}

The construction of these new tables was based on CM predictions correlated with experimental data obtained with a constant flow rate of the heat transfer fluid $\mathrm{HT}-43$ at $0.125 \mathrm{~kg} / \mathrm{sec}(3 \mathrm{gpm})$. Thereafter, correlations at $0.042 \mathrm{~kg} / \mathrm{sec}$ ( $1 \mathrm{gpm}$ ) for heating and cooling and at $0.305 \mathrm{~kg} / \mathrm{sec}(7.3 \mathrm{gpm})$ for cooling were produced.

Table II shows the conditions of nine of the tests which were run, each of which ran for a total of six hours, except for No. 9 which ran four hours. Data were taken at the start of each test and thereafter at one-half hour intervals. Correlations for each data set were made with CM predictions. From these, the set showing the greatest deviation, and the starting condition, were selected. The graphs for these are to be found in Appendix B. In these graphs the symbols have the following meanings:

$$
\begin{aligned}
\Delta & =\text { temperatures measured in deep wells, } \\
0 & =\text { temperatures measured in shallow wells, } \\
+ & =\text { temperatures prediced by the computer } \\
& \text { model. }
\end{aligned}
$$

Figures B-1 - B-4 show the deviation using Enthalpy Table 4. The effect of hysteresis (which is not accounted for in the $\mathrm{CM}$ ) is shown by the fact that Thermkeep temperatures in the temperature range of latent heat are higher than predicted during heating, and lower during cooling.

Figures $B-5$ and $B-6$ show considerably better correlation for heating through the use of Enthalpy Table 7 in the $\mathrm{CM}$, rather than Table 4. 
TABLE II

TEST CONDITIONS FOR HYSTERESIS STUDY CORRELATION

\begin{tabular}{|c|c|c|c|c|}
\hline $\begin{array}{l}\text { Test } \\
\text { No. }\end{array}$ & $\begin{array}{l}\text { Figures of } \\
\text { Appendix B }\end{array}$ & Mode & $\begin{array}{l}\text { HT- } 43 \\
\text { Flow Rate }\end{array}$ & $\begin{array}{c}\text { Enthalpy } \\
\text { Table }\end{array}$ \\
\hline 1 & $B-1, B-2$ & Charge & $\begin{array}{l}0.125 \mathrm{~kg} / \mathrm{sec} \\
(3 \mathrm{gal} / \mathrm{min})\end{array}$ & 4 \\
\hline 2 & $B-3, B-4$ & Discharge & $\begin{array}{l}0.125 \mathrm{~kg} / \mathrm{sec} \\
(3 \mathrm{gal} / \mathrm{min})\end{array}$ & 4 \\
\hline 3 & $B-5, B-6$ & Charge & $\begin{array}{l}0.125 \mathrm{~kg} / \mathrm{sec} \\
(3 \mathrm{gal} / \mathrm{min})\end{array}$ & 7 \\
\hline 4 & $B-7, B-8$ & Discharge & $\begin{array}{l}0.125 \mathrm{~kg} / \mathrm{sec} \\
(3 \mathrm{gal} / \mathrm{min})\end{array}$ & 9 \\
\hline 5 & $B-9, B-10$ & Charge & $\begin{array}{l}0.042 \mathrm{~kg} / \mathrm{sec} \\
(1 \mathrm{gal} / \mathrm{min})\end{array}$ & 10 \\
\hline 6 & $B-11, B-12$ & Discharge & $\begin{array}{l}0.042 \mathrm{~kg} / \mathrm{sec} \\
(\mathrm{I} \mathrm{gal} / \mathrm{min})\end{array}$ & 10 \\
\hline 7 & $B-13, B-14$ & Charge & $\begin{array}{l}0.125 \mathrm{~kg} / \mathrm{sec} \\
(3 \mathrm{gal} / \mathrm{min})\end{array}$ & 10 \\
\hline 8 & $B-15, B-16$ & Discharge & $\begin{array}{l}0.125 \mathrm{~kg} / \mathrm{sec} \\
(3 \mathrm{gal} / \mathrm{min})\end{array}$ & 10 \\
\hline 9 & $\mathrm{~B}-17, \mathrm{~B}-18$ & Discharge & $\begin{array}{l}0.305 \mathrm{~kg} / \mathrm{sec} \\
(7.3 \mathrm{gal} / \mathrm{min})\end{array}$ & 10 \\
\hline
\end{tabular}


Figures B-7 and B-8 show better correlation for cooling through the use of Enthalpy Table 9 in the CM, rather than Table 4.

Figures B-13 - B-16 show the maximum deviation obtained with Enthalpy Table 10 used both for heating and cooling.

The remaining figures show the maximum deviations measured at higher and lower flow rates, using Enthalpy Table No. 10.

On the basis of these tests, it appears that the use of Table 10 for both heating and cooling provides an adequate representation of performance for the purposes of this program. Table 10 was subsequent]y used to correlate all data from the heating and cooling cycling tests, and selected data obtained during the prior program with T-66 as the heat transfer fluid.

Some preliminary consideration has been given to the problems of incorporating into the computer model a means for accounting for the apparent hysteresis effect. However, this appears to be very complex, and to require quantitative information not now available. The true nature of the phenomenon is not understood. A supercooling and/or superheating may be involved in which the temperatures of latent heat effects are elevated during heating, and/or depressed during cooling. The degree of elevation or depression may be functions of the temperature and/or the rate of heating or cooling. A contributing factor may stem from the fact that upon cooling from the liquidus range, the first solid which forms on the heat exchanger is pure $\mathrm{NaOH}$ whose melting point is given by literature references as $593 \mathrm{~K}$ (cf Fig. 5), which is above the temperature of the heat transfer fluid entering during charging. Upon reheating with a heat transfer fluid whose temperature is several degrees below its melting point, reliquefying must be principally by solution in the liquid phase at the liquid-solid interface rather than at the heat exchanger surface. (The $\mathrm{CM}$ is based upon this mechanism.)

Experimentally determined information would be needed relating the rate of change of the enthalpy of the Thermkeep to its temperature, its rate of heating or cooling, and, if necessary, its immediately preceding temperature history. This information may be extractable from the experimental data already in hand, which 
includes an almost continuous temperature history of Thermkeep temperatures throughout the TES unit for a wide range of heating and cooling rates, and for cycles in which heating is immediately followed by cooling, and vice versa.

If the necessary information can be obtained, it must then be introduced into the $\mathrm{CM}$ in a way which follows the first law, the conservation of energy. That is, when an element of Thermkeep is carried through a cycle within the temperature range of the hysteresis effect, the cycle must form a closed loop on the temperatureenthalpy diagram. This cannot be accomplished simply by using two tables, one for heating and one for cooling and shifting from one to the other. For example, with reference to Figure 6 , if Table 7 were used for heating and Table 9 for cooling, and if an element were heated from $558 \mathrm{~K}$ to $573 \mathrm{~K}$ along curve 7 , then cooled along curve 9 back to $558 \mathrm{~K}$, more energy would be delivered by the element during cooling than would be rèceived during heating. Upon repeated cycling between these temperatures, the element would progressively lose energy.

This might be avoided by introducing a third $t-h$ relationship such as Table 10 which represents a zero-rate relation between $t$ and $h$, and by requiring that the maximum and minimum temperatures of cycles which are within the hysteresis temperature range must lie on this curve.

However, correlations next to be described, in which Enthalpy Table 10 was used for all cycles are believed to be adequate for the intended uses of the $\mathrm{CH}^{\pi}$.

\section{Data Correlation}

The data correlations consisted of comparisons between experimental data (DATA) and computer predictions (CMP). The correlations include Thermkeep (TK) temperatures and the temperatures of the heat transfer fluids entering or leaving the upper end of the heat exchanger (TU) and entering or leaving the lower end (TL). 
Correlations were made using data for Therminol-66

(T-66) from the prior program and for Caloria HT-43 (HT-43) obtained in the present program. The results are given in Appendix $C$ in graphical form.

Figures $\mathrm{C}-1$ through $\mathrm{C}-12$ show $\mathrm{HT}-43$ inlet and outlet temperatures vs. time. Figures $\mathrm{c}-13$ through $\mathrm{C}-48$ show the corresponding Thermkeep temperature profiles in the tank.

Figures $\mathrm{C}-49$ through $\mathrm{C}-59$ show $\mathrm{T}-66$ inlet and outlet temperatures vs. time. Figures $\mathrm{C}-60$ through $\mathrm{C}-75$ show the corresponding Thermkeep temperature profiles in the tank.

The tests performed consisted of four types:

1. Single discharge, in which the TES is initially charged, and is discharged by a constant flow of fluid.

2. Single charge, in which the TES is initially in a partially charged state, as at the end of a typical cycle, and is charged by a constant flow of fluid.

3. Cyclic, in which a cycle consists of a 4-hour charge and a 4-hour discharge at constant fluid rate.

4. Solar cycle, which conforms to the solar daily cycle described in NASA CR-159465. The solar daily cycle is shown in Figure 7 .

The conditions of the tests performed in the present program are given on Table III.

The conditions of the prior tests with T-66 are shown on Table IV. The correlation of the data with CMP is given in NASA CR-159465, which used the original Enthalpy Table 4.

Correlation of the original data for T-66 temperatures with CMP was repeated for inclusion herein, using Enthalpy Table 10, and also TK temperature correlations for Test 018 and Test 023 . 


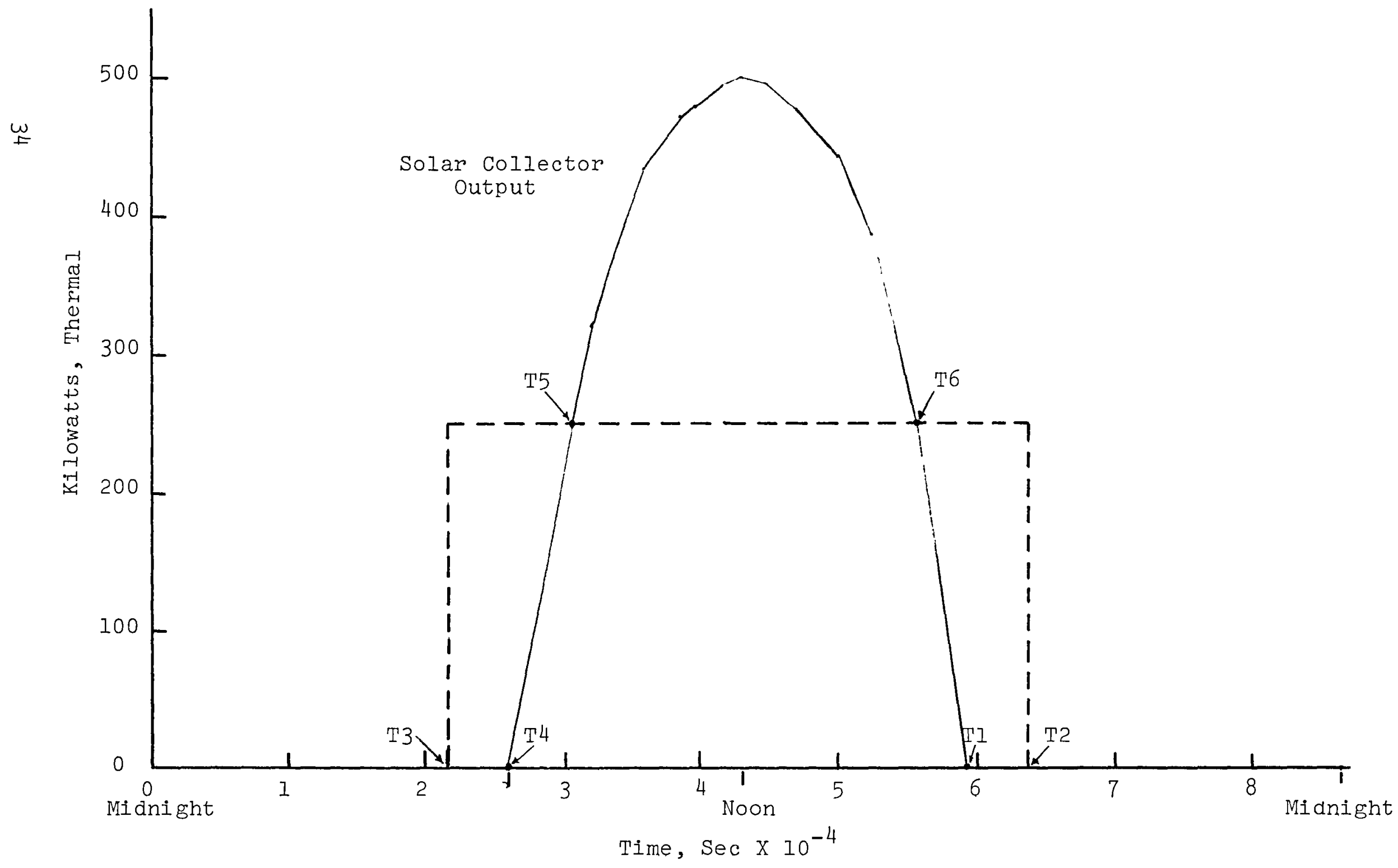

Figure 7. Typical solar daily cycle at Sandia Total Energy Test Facility. 
TABLE III

TESTS WITH CALORIA HT-43 UNDER PRESENT PROGRAM

Test

No.

201

202

204

214-2

215-2

218

219

223
Type of Test

Single discharge, 6 hrs .

Single charge, 6 hrs.

Single charge, 6 hrs.

Single discharge, 6 hrs.

Single charge, 6 hrs.

Cyclic; 4-8 hr. cycles

Single discharge, 4 hrs.

Solar cycle; 2-12 hr. cycles
HT-43 Flow Rate

$\mathrm{kg} / \mathrm{sec}$

gpm

0.14

$3 \cdot 3$

0.14

$3 \cdot 3$

0.16

$3 \cdot 7$

0.046

1.1

0.046

1.1

0.080

1.9

0.32

$7 \cdot 7$
TABLE IV

TESTS WITH THERMINOL-66

UNDER PRIOR CONTRACT*

Test

No.

001

002

004

014

015

018

019

023
Type of Test

Single discharge, 6 hrs .

Single charge, 9 hrs.

Single charge, 6 hrs.

Single discharge, 6 hrs.

Single charge, 6 hrs.

Cyclic; $3-8$ hr. cycles

Single discharge, 3 hrs.

Solar cycle; 2-12 hr. cycles
T-66 Flow Rate $\mathrm{kg} / \mathrm{sec}$

$\mathrm{gpm}$

0.15

2.9

0.15

2.8

0.18

3.4

0.053

1.0

0.053

1.0

0.106

2.0

0.35

6.6

Variable

* See NASA CR-159465 
For the constant rate charging and discharging tests with HT-43, TK temperature profiles are shown for the initial state, and after 3 hours and 6 hours, except in the case of Test 219 which was of shorter duration. After 6 hours, correlation is generally good for flow rate of $0.14 \mathrm{~kg} / \mathrm{sec}$. An exception is noted in the case of Test 204 , at $0.16 \mathrm{~kg} / \mathrm{sec,} \mathrm{which} \mathrm{appeared} \mathrm{be-}$ tween the 4 th and 5 th hours, the cause of which is unexplained. The correlation of HT-43 temperatures for all these tests is good, with a maximum discrepancy $t$ the end of the discharging tests of $6 \mathrm{~K}$ (Test 201). The HT-43 temperature correlation of Test 204 is good ( $4 \mathrm{~K}$ maximum discrepancy) in spite of the discrepancy noted above in the TK temperature correlation.

The high constant discharge rate test (Test 219) showed more discrepancy in the TK temperatures than the lower rate tests. However, The HT-43 temperature correlation is exceptionally good, the maximum discrepancy being only about $1 \mathrm{~K}$.

The cyclic test with HT-43 (Test 218) consists of 4 cycles, each consisting of 4 hours of discharging followed by 4 hours of charging at a constant flow rate of $0.080 \mathrm{~kg} / \mathrm{sec}(1.9 \mathrm{gpm})$. During the discharge portions of this test, the same type of discrepancy occurred as in Test 204. This is reflected in the HT-43 temperature graphs; however, the maximum discrepancies between DATA and CMP were less than $10 \mathrm{~K}$ at the end of 32 hours of cycling.

Test 223 shows the results of the solar cycle test with HT-43. Although some discrepancy is apparent in $\mathrm{TK}$ temperatures between 6 and 8 hours, they disappear at 10 hours. Deviations between DATA and CMP for HT-43 temperatures are no greater than about $5 \mathrm{~K}$ throughout the 36 hour duration of the test.

The TK temperatures of the cyclic Test 018 using T-66 show better correlation than that of Test 218 using $\mathrm{HT}-43$, and this is reflected in a very good correlation for the Test 018 fluid temperatures. On the other hand, the correlation of fluid temperatures for the Solar Cycle tests (Tests 223 and 023) is better for HT-43 than for T-66. The Solar Cycle test with T-66 shows approximately the same degree of correlation with Enthalpy Table 10, as with the previously reported Enthalpy Table 4. 
In view of the generally good correlation of the fluid temperatures of the steady flow rate tests, it is surprising that the discrepancy is so high in the solar cycle with T-66. It is noted that the large discrepancy occurs during the decreasing fluid flow portion of the charge phase of both the first and second cycles, and that the correlation is good during the discharge portion of both cycles (hours 10-12 and 34-36) which follows the charge portions (hours 3-9 and 27-33). Since the fluid flow rate during charging is variable in the solar cycle (starting and ending at substantially zero), it may be that the experimental model does not perform as assumed in the computer model. The latter assumes uniform flow through all 25 heat exchanger elements and that there are no lateral temperature variations within the elements. There is evidence that this was not the case during the periods in which the discrepancy occurs.

Figure 75 of Appendix $C$ shows temperatures at the surface of the tank ( $D$ ), in the shallow wells (o), and in the deep wells $(\Delta)$, at 32 hours of the solar cycle Test 023 , using T-66. Temperature variations of as much as $25 \mathrm{~K}$ are noted between the surface and deep well locations, and about $13 \mathrm{~K}$ between the deep and shallow well locations. Similar variations were recorded at the 9 th hour of the test which is the corresponding point in the first cycle. A similar pattern of variations is apparent in the solar cycle test with HT-43.

These temperature variations may result from variations in fluid flow rates through the corresponding elements of the heat exchanger. If this is the case, it will undoubtedly be correctable by manifolding design changes.

\section{Summary}

The practical significance of this work lies in the correlation of the measured heat transfer fluid outlet temperature with that predicted by the computer model. For the six constant rate tests of charging and discharging with $\mathrm{HT}-43$, the average difference between experimental and predicted temperatures is $3 \mathrm{~K}$, and the maximum is $6 \mathrm{~K}$. For T-66, the average is $2 \mathrm{~K}$ and the maximum is $7 \mathrm{~K}$. For the constant flow cycle tests (218 and 018), the discrepancy at the end of 3 cycles is $6 \mathrm{~K}$ for $\mathrm{HT}-43$ and $2 \mathrm{~K}$ for $\mathrm{T}-66$. For the solar cycle with T-66, a maximum discrepancy of $14 \mathrm{~K}$ was noted, while for $\mathrm{HT}-43$, the maximum was only $5 \mathrm{~K}$. 
It is believed that the computer model is reliable enough to be used in the design of phase-change TES systems, using Thermkeep as the heat storage medium, and nonphase-change heat transfer liquids which are generally similar in properties to HT-43 and T-66. 
PART III

DUAL HEAT EXCHANGE DESIGN ANALYSIS

Introduction

Early investigations into the use of Thermkeep* as a phase-change heat storage medium for solar power generation systems suggested that a more economical storage unit could be produced if both the collector fluid (T-66) and the working fluid (Toluene) of the power cycle were caused to flow through the storage unit. This would eliminate the cost of a separate boiler but would increase the cost of the heat exchanger within the storage unit.

It was felt that the thermodynamics of the storage and retrieval process would be superior in the case of the two-fluid storage unit because of the inherent irreversibilities and large $\Delta T ' s$ associated with the fluid pinchpoints which occur when heat is exchanged between the non-phase change fluid (T-66) and the phase-change storage medium and power fluid. Early attempts to quantify this did indeed indicate a substantial benefit in terms of overall storage unit size for acceptable performance with the use of a two-fluid storage device.

For this program, an analysis was developed which accounts for the use of two fluids in a typical daily duty cycle according to which the solar collector fluid flows during hours of collection at a rate determined by the insolation intensity. Moreover, the power fluid, which is a vaporizing fluid such as Toluene, flows during the hours of power generation. Consequently, at some times both fluids flow; at other times only one flows.

\section{$\underline{\text { Approach }}$}

There are two ways to approach the analysis and the first effort in the formulation assessed the relative complications and benefits of the two approaches. As might be expected, the approach which is more precise is considerably more difficult to perform.

* Registered Trademark Comstock \& Wescott, Inc. 
The simpler approach is to view the heat exchange process as one in which each fluid exchanges heat with the storage medium, but not directly with the other. A heat balance is made on the numerical element of storage medium at the end of a time step during which the temperature of the medium is assumed to remain constant. Then an updated temperature distribution is produced.

The other approach which requires additional simplifying assumptions in order to be made solvable is to consider also the direct heat exchange between the two fluids. This permits a substantial amount of heat transfer from the collector fluid to the power fluid without the intervening resistance of the storage medium. It also reduces the quantity of medium required because the medium need have capacity only for the heat stored, rather than all the heat flowing from the collectors to the power fluid.

Direct heat exchange between the collector fluid and the power fluid, when both fluids are in energy balance, has a clear thermodynamic advantage, albeit one which has not yet been quantified. In the existing system configuration, whereby the boiler and storage system exist as two separate components, collector fluid heats the power fluid directly during a substantial part of the daily cycle. Since all the fluid flow in the system under analysis herein must flow through the storage unit, it seemed that a direct heat exchange path between the two fluids would be preferred over one which imposed the heat transfer resistance of the storage medium.

Therefore, the analysis has been formulated to account for simultaneous heat transfer between the fluids and between each fluid and the storage medium, i.e., threeway heat exchange. The purpose of a parametric study would be to evaluate the effect of changes in the heat transfer path resistances between the three media. Moreover, the results of optimization in this configuration would be compared in terms of cost, performance, and operational characteristics to the current standard design which passes only collector fluid through the storage unit and uses a separate boiler to heat the power fluid. 
General Features

The prior analysis may be referred to as a design analysis -- that is to say it includes a rather detailed series of calculations relating to a number of specific characteristics of the storage system. It works from an input set which consists of basic geometry from which it derives heat transfer parameters such as surface areas and pressure drops. It does a cost analysis. It includes axial conduction heat flows and losses to the environment. It is limited, however, to a specific geometry -- that of coiled tubes in a parallel configuration.

At the outset of the present work, it was not known what sort of geometry would be preferred for a threeway system. A determination was made to provide a less detailed analysis which would describe the basic heat transfer and thermodynamic behaviour of the process and to overlook for the moment other effects which have already been shown to be relatively insignificant. On this basis, calculations with regard to geometries, pressure drops, losses to the environment, and axial losses have been put aside.

It will not be difficult to introduce these later if wanted. It will require, however, a clearer, more limiting description of the desired design configuration, one not deemed appropriate at this time. Also, it was not entirely clear at the outset that a problem of such complexity as this would lend itself to a useful solution, and introduction of additional details was not considered prudent.

\section{General Description}

The analysis considers variations in the temperatures of the three media to be one-dimensional; temperatures vary only in the direction of flow of the fluid media. The hot end of the unit is the top, as shown on Figure 8 . This stabilizes the thermal gradient established in the storage medium by preventing mixing due to natural convection flow.

Heated fluid from the solar collector, assumed to be a liquid with a constant specific heat, $C_{I}$, flows through 


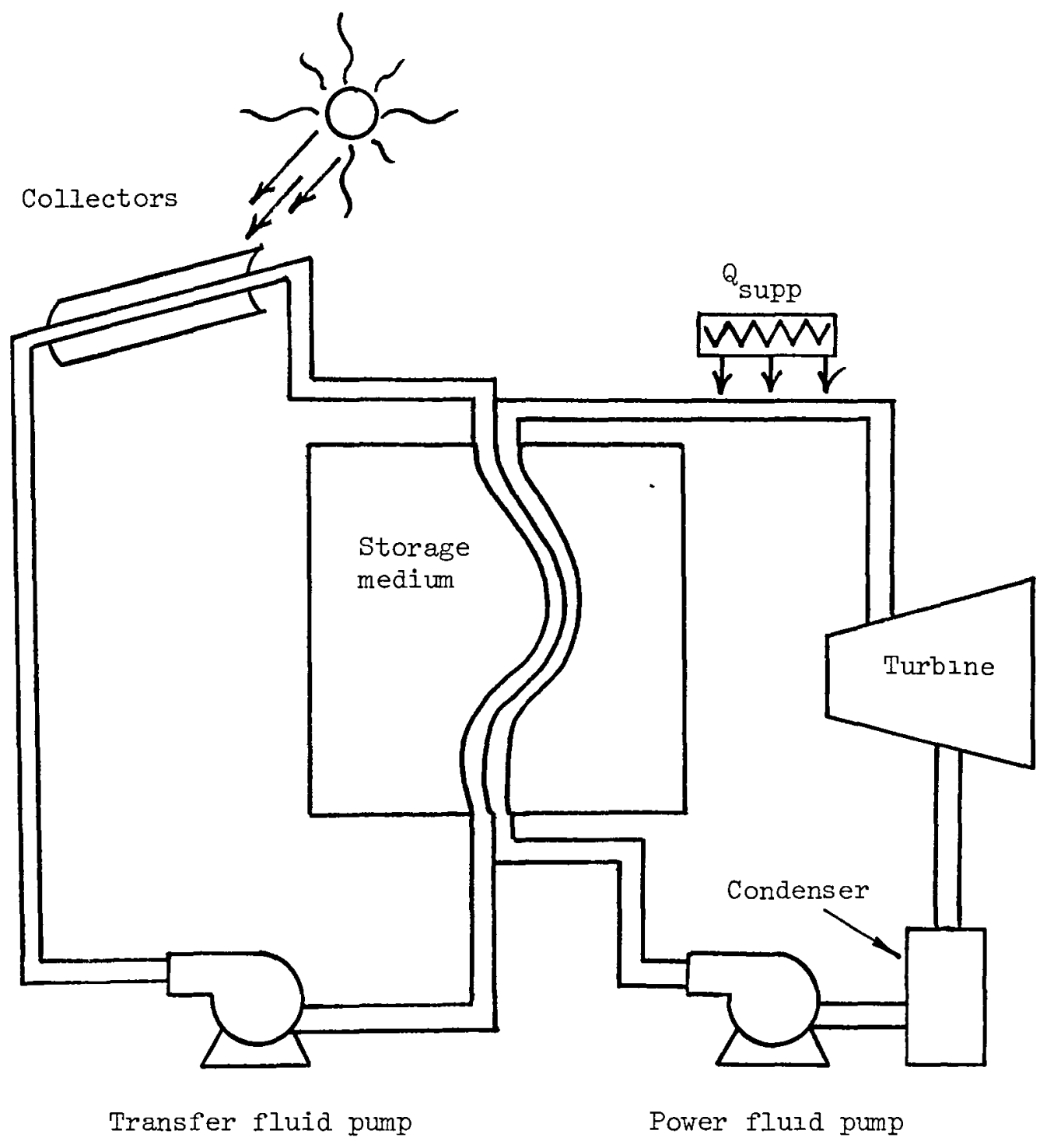

FIgure 8. Solar electric power generating system schematic. 
the unit from top to bottom. Its inlet temperature, assumed to be constant, is $\mathrm{T}_{\mathrm{L} \text {, in }}$ while its exit

temperature is $\mathrm{T}_{\mathrm{L} \text {, out }}$. Its flow rate, also a time variable, is $\mathrm{W}_{\mathrm{L}}$, its value being a function of the collector heat output and the fluid temperature.

Cooled power fluid, assumed to be a vaporizing liquid with constant specific heats in the sensible regions, flows into the unit at the bottom and out at the top. Its Iiquid phase specific heat is $C_{S}$, its vapor phase specific heat is $C_{V}$, its heat of vaporination is $Q_{T}$, its flow rate is $W_{P}$ (assumed to be constant in time), its inlet temperature is $T_{P}$, in (constant in time) and its exit temperature is $\mathrm{T}_{\mathrm{P}}$,out which is a time-varying output of the analysis.

The power conversion system, however, in order to produce a constant output, $P$, requires a constant inlet temperature, $\mathrm{T}_{\mathrm{P}}$, des, and a boost heater is assumed to make up the difference between the desired and the actual delivery temperatures. In general, this supplemental heat is

$$
\mathrm{Q}_{\text {supp }}=\mathrm{W}_{\mathrm{P}} \mathrm{C}_{\mathrm{V}}\left(\mathrm{T}_{\mathrm{P}, \text { des }}-\mathrm{T}_{\mathrm{P}, \text { out }}\right)
$$

The solar collectors produce a scheduled amount of heat, $Q_{c o l l}$, which is assumed to be a function of time of day but repeatable from day to day. The collector fluid attempts to absorb this heat by varying its flow rate according to

$$
W_{L}=\frac{Q_{\operatorname{coll}}}{C_{L}\left(T_{L, \text { in }}-T_{L, \text { out })}\right.}
$$


At some point, the fluid pump reaches a maximum flow, $W_{L, \max }$, and any heat which cannot be absorbed due to this Iimiation is assumed to be lost through a heat rejection device. It is calculated by

$$
Q_{\text {Iost }}=Q_{\text {coll }}-W_{L, \max } C_{L}\left(T_{L, \text { in }}-T_{L, \text { out }}\right)
$$

Both $Q_{\text {Iost }}$ and $Q_{\text {supp }}$ are important operational characteristics of the system.

The task of the analysis is to use as input the parameters $Q_{\text {coll }}, P, T_{L, i n}, T_{P, i n}$, the fluid thermal properties, and the storage unit geometry, and to calculate $\mathrm{T}_{\mathrm{L} \text {, out }}, \mathrm{T}_{\mathrm{P} \text {, out }} \mathrm{Q}_{\text {lost, }} \mathrm{Q}_{\text {supp, }}$, and the storage medium temperature variation in space and time. The material discussed so far, the parameters external to the storage unit itself, constitutes the essence of the MAIN portion of the computer analysis. The major portion of the computer analysis, a subroutine called ELANAL, deals with the processes within the storage unit. It receives the flows and inlet temperatures of the fluids from the MAIN program.

Heat Exchange Analysis

Description

The analysis of the heat exchange processes within the storage unit rests upon the same assumptions made to develop the heat exchange analysis for the single fluid case. The actual procedure is greatly complicated by the fact that two fluids flow countercurrent and that one fluid exists in three states: subcooled liquid, two-phase boiling fluid, and superheated vapor. This last factor imposes the definition of three distinct spatial regions: Region I where the power fluid is superheated, Region II where boiling is taking place, and Region III where the power fluid is subcooled. 
Two basic approximations are made in the solution methodology; finite differencing in time, and finite elements in space. With regard to the temporal variation in the parameters, the approximation is made that the time axis is divided into sufficiently small time steps that the system may be viewed as stationary during said time steps.

Heat flows are computed from temperature potentials assumed not to change significantly during the time step. The heat flows contribute to a stepwise change in the thermal state of the storage medium, the result of which is assumed staionary for the next time step. The storage medium is assumed to have the only thermal capacitance of any significance.

Thus, the differential equation

$$
Q=M C \frac{d T}{d t}
$$

is approximated by

$$
\Delta \mathrm{T}=\frac{\mathrm{Q}}{\mathrm{MC}} \Delta \mathrm{t}
$$

Where the $\Delta T$ of a piece of storage medium of mass, $M$, and specific heat, $C$, is that created by imposing a fixed heat flow $Q$ on it over a time step, $\Delta t$. This is the finite differencing in time.

Spacial variation of the temperature of the storage medium is approximated by a stepwise variation in the (vertical) $\mathrm{X}$-direction. The unit is divided in the $\mathrm{X}$-direction into $\mathrm{N}_{\mathrm{E}}$ elements of size $\Delta \mathrm{X}$ and of mass $\Delta \mathrm{M}$. Figure 9 describes an element. Each element is assumed to be of uniform temperature, $\mathrm{T}_{i}$. For each element, heat flows among the media are computed from the knowledge of the inlet temperatures of each fluid, $\mathrm{T}_{\mathrm{H}, i+1}$ and $\mathrm{T}_{\mathrm{C}, i}$, the 


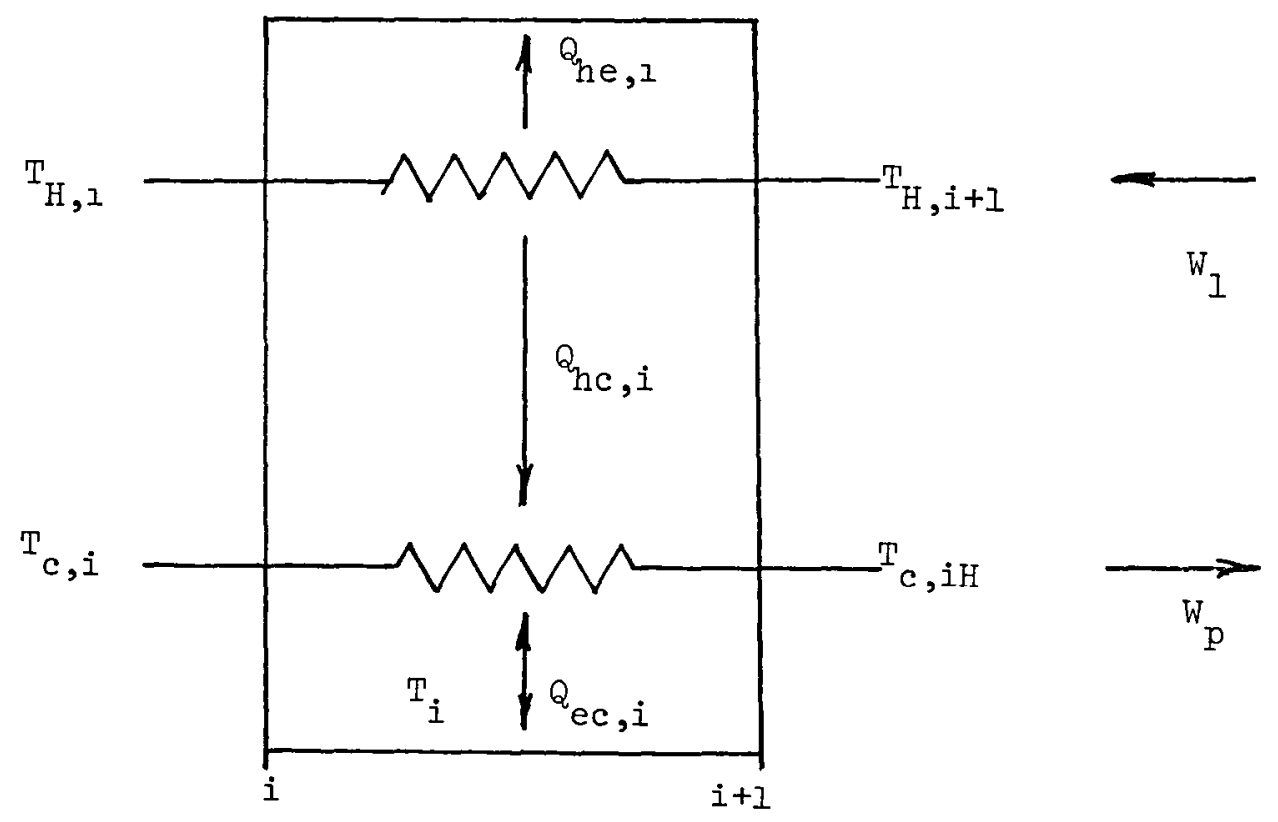

Figure 9. Element for heat exchange analysis, 
element temperature, $\mathrm{T}_{i}$, and the heat flow resistances between the media.

The heat flow between the fluid media, $Q_{\mathrm{HC}, i}$, contributes a portion of the heat needed to change the fluid temperatures (or states). The heat flows between the storage medium and each fluid contribute the remainder. These heat flows, $Q_{H E, i}$ and $Q_{E C, i}$, also, when summed together constitute the net heat flow to the storage medium finite element and, when multiplied by the appropriate time step, $t$, determine the element temperature change. Consequently, the updated set of element temperatures may be calculated to be used in the next time step.

The three-way heat flow analysis for the power fluid is different in the sensible regions, where temperature changes with heat exchange, from that in the boiling region, where quality changes with heat exchange. Consequently, the normally equally sized elements must be divided into fractional elements when a regional transition occurs, each fraction being described by its appropiate relationships.

\section{Governing Equations}

For any element with constant element temperature and fluids flowing counter-currently, the following equations describe the heat transfer and thermodynamics:

$$
\begin{aligned}
& d T_{H}(X)=-\frac{d Q_{H E}+d Q_{H C}}{W_{L} C_{L}} \\
& d T_{C}(X)=-\frac{d Q_{E C}-d Q_{H C}}{W_{P} C_{C}}
\end{aligned}
$$




$$
\begin{aligned}
& \mathrm{dQ}_{\mathrm{HE}}=(\mathrm{UA})_{\mathrm{HE}}\left(\mathrm{T}_{\mathrm{H}}-\mathrm{T}\right) \mathrm{dX} \\
& \mathrm{dQ}_{\mathrm{HC}}=(\mathrm{UA})_{\mathrm{HC}}\left(\mathrm{T}_{\mathrm{H}}-\mathrm{T}_{\mathrm{C}}\right) \mathrm{dX} \\
& \mathrm{dQ}_{\mathrm{EC}}=(\mathrm{UA})_{\mathrm{EC}}\left(\mathrm{T}-\mathrm{T}_{\mathrm{C}}\right) \mathrm{dX}
\end{aligned}
$$

where (UA) ${ }_{\mathrm{HE}}$ is the product of $U$, the overall heat transfer coefficient, and $A$, the effective surface area for heat transfer, associated with the heat flow $Q_{H E}$ and likewise for $(U A)_{H C}$ and $(U A)_{E C}$. The UA are derived in the normal way considering the overall heat flow path between the two relevant media.

There are two solution forms of use to this situation. In the general case the power fluid is in the sensible region and $\mathrm{T}_{C}$ is, in fact, a function of $x$. In this case, the solution is of the form

$$
\begin{aligned}
& T_{C}(X)=z_{1} C_{1} e^{\left(\frac{\lambda_{1} X}{L}\right)}+z_{2} C_{2} e^{\left(\frac{\lambda_{2} X}{L}\right)}+z_{3} \\
& T_{H}(X)=c_{1} e^{\left(\frac{\lambda_{1} X}{L}\right)}+c_{2} e^{\left(\frac{\lambda_{2} X}{L}\right)}+z_{4}
\end{aligned}
$$

where $\lambda_{1}, \lambda_{2}$ are solutions to a characteristic equation; $L$ is the effective element length; $Z_{1}$, $z_{2}, z_{3}$, and $z_{4}$ are groups of terms formed from the 
UA, $W_{L} C_{L}, W_{P} C_{C}$, and the element temperature, $T$, which is constant. $\mathrm{C}_{1}$ and $\mathrm{C}_{2}$ are constants determined by substitution of the boundary conditions, i.e., the known inlet temperatures.

For the more specific case of $\mathrm{T}_{C}(X)$ being constant, which describes the boiling nrocess occurring at saturation temperature, the solution for $\mathrm{T}_{\mathrm{H}}(\mathrm{X})$ is of form:

$$
T_{H}(x)=z_{5} e^{\left(\frac{\lambda_{3} X}{L}\right)}+z_{6} .
$$

Heat flows for all paths may be calculated by integration of the heat flow equations once the temperature expressions are known. For example,

$$
{ }_{H C}=(U A)_{H C} \int_{0}^{L}\left(T_{H}(X)-T_{C}(X)\right) d X
$$

Application

In subroutine ELANAL, the element heat flows are determined from relationships arising from the basic equations discussed above. It is to be noted, however, that the fluid temperatures entering each element are initially unknown. In fact, all that are known are temperatures, $\mathrm{T}_{\mathrm{C}, 1}=\mathrm{T}_{\mathrm{p} \text {, in }}$ and $\mathrm{T}_{\mathrm{H}, \mathrm{NE}+\mathrm{I}}=\mathrm{T}_{\mathrm{I} \text {,in }}$ from the external system. Not only that, there are three distinct regions, previously described, the boundaries of which are not known until solution is achieved. 
It can be shown that a set of elements totally composed of fluids in uniquely sensible regions can be solved for directly through simultaneous solution of a set of linear equations in the unknown fluid temperatures at the element boundaries.

This is suggested by the fact that $N_{E}$ elements yield $2 \mathrm{~N}_{\mathrm{E}}$ equations for temperatures of which there are $2 \mathrm{~N}_{\mathrm{E}}+2$, of which 2 are the known inlet temperatures for each fluid. In fact, the matrix of coefficients, if the equations are properly arranged, is a tridiagonal matrix, and special computer subroutine packages exist for this situation.

The computer analysis proceeds in the following way:

- A position is assumed for the point where the power fluid reaches a quality of 1.0 . Here, the temperature is $\mathrm{T}_{\text {sat }}$, the saturation temperature, and all elements above this point lie in a region where the sensible heat equations govern. The inlet temperature to this set of elements is known for the collector fluid as well since it is $\mathrm{T}_{\mathrm{H}, \text { in }}$.

. The aforementioned solution is used for the set of linear equations. This yields all the desired fluid temperatures including the collector fluid temperature at the Xcoordinate corresponding to power fluid quality of 1.0 , hereafter called $\mathrm{T}_{L F}$.

- Knowing the two associated fluid temperatures at the end of the boiling region, the heat flow equations for $\mathrm{T}_{\mathrm{C}}=$ constant may be used to work backwards to the point where quality is exactly 0.0. Neither this point nor the point where quality is 1.0 necessarily fall on an element boundary. Not only will the point be determined but also the associated collector fluid temperature, called hereafter $\mathrm{T}_{\mathrm{LI}} \cdot$ 
.. The region below this point will consist of power fluid entirely in the sensible region and may be solved for again by the set of simultaneous linear equations and knowledge of $\mathrm{T}_{\mathrm{LI}}$ and $\mathrm{T}_{\mathrm{P} \text {, in }}$. This will, however, result in a calculated temperature for the power fluid at the outlet, where a quality of 0.0 was expected, which will differ from $T_{\text {sat }}$ The extent to which it differs is a measure of the accuracy of the choice of the point where quality is identically 1.0 .

There are two approaches to the use of this method. One may be called a closed loop approach and the other an open loop approach. The closed loop approach is an iterative approach whereby knowledge of the error in temperature match where quality is to be 0.0 is used to adjust the point where quality is 1.0. Adjustment proceeds until the error falls within acceptable limits. This iterative approach is more accurate but more costly in terms of computer processing time.

The open loop approach uses a method of prediction of the point where quality is 1.0 and assumes that by proper selection of the prediction method a satisfactory accuracy will be achieved. The cost of such an approach is much less but accuracy is apt to be sacrificed. This is the approach taken and results to date are considered satisfactory. A method of tracing the movement of the saturated vapor point has been developed which combines a knowledge of the current error with a predictor of the expected result of having updated element temperatures.

Appendix $E$ is a listing of the Fortran program used for this work.

\section{Performance Analysis}

A design is specified by certain limited input parameters. The surface areas associated with each heat flow path, as well as heat transfer film coefficients for each fluid define the geometry. The collector fluid film coefficient is input as a function subprogram whose value depends upon fluid flow rate. Since the power fluid flow rate is fixed 
over a cycle, the only variation provided by the analysis is a dependence upon quality in the two-phase region.

The duty cycle is, as in the single fluid situation, such that the solar collectors produce a normal daily variation in output, peaking at solar noon. The power system operates for a certain part of the cycle, in the early morning and early evening, when the collector output is zero. Overall the collectors can provide more over a day than the power cycle needs and storage smooths the daily variation in collector output in an attempt to provide constant heat input required.

Each design run is accomplished by setting the unit at full charge, i.e., uniformly at peak cycle temperature, and letting the daily cycles progress until heat input and output are equal within acceptable limits. This stable repetitive operation is the mode of operation assumed to be characteristic of the design. Once stability is achieved, then the important rating criteria can be established.

When equilibrium is established, the capacity of the unit is available as the daily "Storage Output." A "Figure of Merit" is defined as the Storage Output divided by the sum of the Storage Output and the supplemental heat,

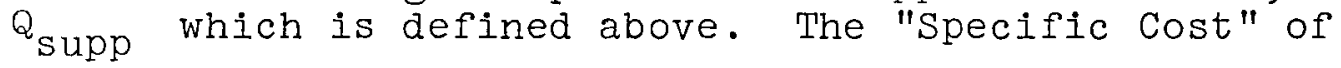
the TES unit is defined as the total cost divided by that portion of the daily output which passes through the storage medium.

An analysis has been used to determine the expected performance of such a system and the results appear in the sections to follow. Also, the optimal design from earlier work for a single fluid system has been rerun with the higher order effects such as insulation heat loss and axial heat conduction within the storage unit eliminated, so that the two systems are compared on a common basis.

The Fortran code of the computer model and notes on its use are to be found in Appendix $D$. 
Results of Computer-Model-Predicted TESS Designs

The purpose of the dual heat exchanger TESS design exercise was to produce a cost optimized design of the dual heat exchanger TESS for comparison with the cost optimized design of the single heat exchanger TESS reported in NASA CR-159465. The single heat exchanger TESS requires the use of an external heat exchanger to vaporize and superheat the power fluid, whereas in the dual heat exchanger TESS this function is incorporated internally and the external heat exchanger is eliminated. Therefore, the cost of the dual heat exchanger design is credited with the cost of the external heat exchanger. This is done in two steps; that is, the specific costs of the two designs are compared with, and without, the external heat exchanger credit. Additional savings in operating costs in the solar-electric system which accrue to the dual heat exchanger design are pointed out, but not credited.

The specifications of the TESS design are as follows:

Storage capacity is $3.1 \times 10^{6} \mathrm{~kJ}$ when operating over the range $516 \mathrm{~K}$ to $584 \mathrm{~K}$. Heat is charged into storage medium (Thermkeep) by the non-phase change heat transfer fluid, Caloria $\mathrm{HT}-43$ at a maximum rate of $1.8 \times 10^{6}$ $\mathrm{kJ} / \mathrm{h}$ at an entering temperature of $584 \mathrm{~K}+2 \mathrm{~K}$. Heat is discharged from storage by means of toluene which enters as liquid at $473 \mathrm{~K}$, and leaves as superheated vapor at $582 \mathrm{~K}+2 \mathrm{~K}$ and $1,862 \mathrm{kPa}$. The maximum heat discharge rate is $1.0 \times 10^{6} \mathrm{~kJ} / \mathrm{h}$. The TESS includes an auxiliary heater which supplies auxiliary heat to raise the temperature of the toluene vapor to the specified temperature as required. The Figure of Merit (FOM) is the fraction of the heat delivered which comes from storage.

Using the computer program described in Appendix $D$ (Use of Computer Program), trial designs were run on the Solar Cycle running through consecutive cycles to produce stabilization. These were chosen for convenience to have power fluid and heat transfer fluid heat exchangers of equal area, the inside heat exchanger surfaces of each varying from $37.2 \mathrm{~m}^{2}$ to $148.6 \mathrm{~m}^{2}$ ( 400 to $1600 \mathrm{ft}^{2}$ ) in increments of $18.6 \mathrm{~m}^{2}\left(200 \mathrm{ft}^{2}\right)$. Note that these are 
the inside areas of each of the two heat exchangers. Thermkeep masses were varied in increments of $1 \mathrm{Mg}$ between 10 and $25 \mathrm{Mg}$. A limitation imposed upon all designs was that the TESS always furnish power fluid of quality 1.0 vapor at the outlet; if it fell below this, the program was terminated.

For a given heat exchanger design, a minimum Thermkeep mass was found below which the outlet vapor quality dropped below 1.0. For each design a storage specific cost and a Figure of Merit (FOM) were calculated.

For comparison purposes the single-tube heat exchanger design program previously developed (NASA CR-159465) was rerun with several program changes to provide the same operating conditions as in the dual heat exchanger design; viz, axial conduction and insulation loss were eliminated, the Thermkeep properties were taken from temperature-enthalpy Table 10 (see Part II), and a return temperature was calculated for the heat transfer fluid flow from the TESS combined with that from the external power fluid boiler, the results of which are shown on Figure 10. The Fortran code of the modified program is given in Appendix E. As a result of the modifications, the FOM rose from .88 to .92 , and the specific cost decreased from $\$ 11.20$ to $\$ 9.57 / \mathrm{MJ}$, as compared with the values given in NASA CR-159465.

The cost of each unit was computed using identical material and fabrication costs, tube wall thickness $(.0004 \mathrm{~m})$, insulation thickness $(.6096 \mathrm{~m})$, aspect ratio $(1.0)$, and vessel wall and bottom thickness $(.0127 \mathrm{~m}$ and $.0254 \mathrm{~m}$, respectively).

Input data consist of the cost of material for each component and costs for fabrication on the basis of weight and surface area. Material costs were those at the time of construction of the experimental model in 1977. These fabrication costs are based upon the actual manufacturing cost of two experimental prototype "Thermbank" electric water heaters constructed at that time of the type described in the background section of the Introduction. Therefore, they do not include the cost benefits of the "learning curve" which results from converting prototype designs to manufacturing designs nor the cost reductions which result from quantity manufacturing. 


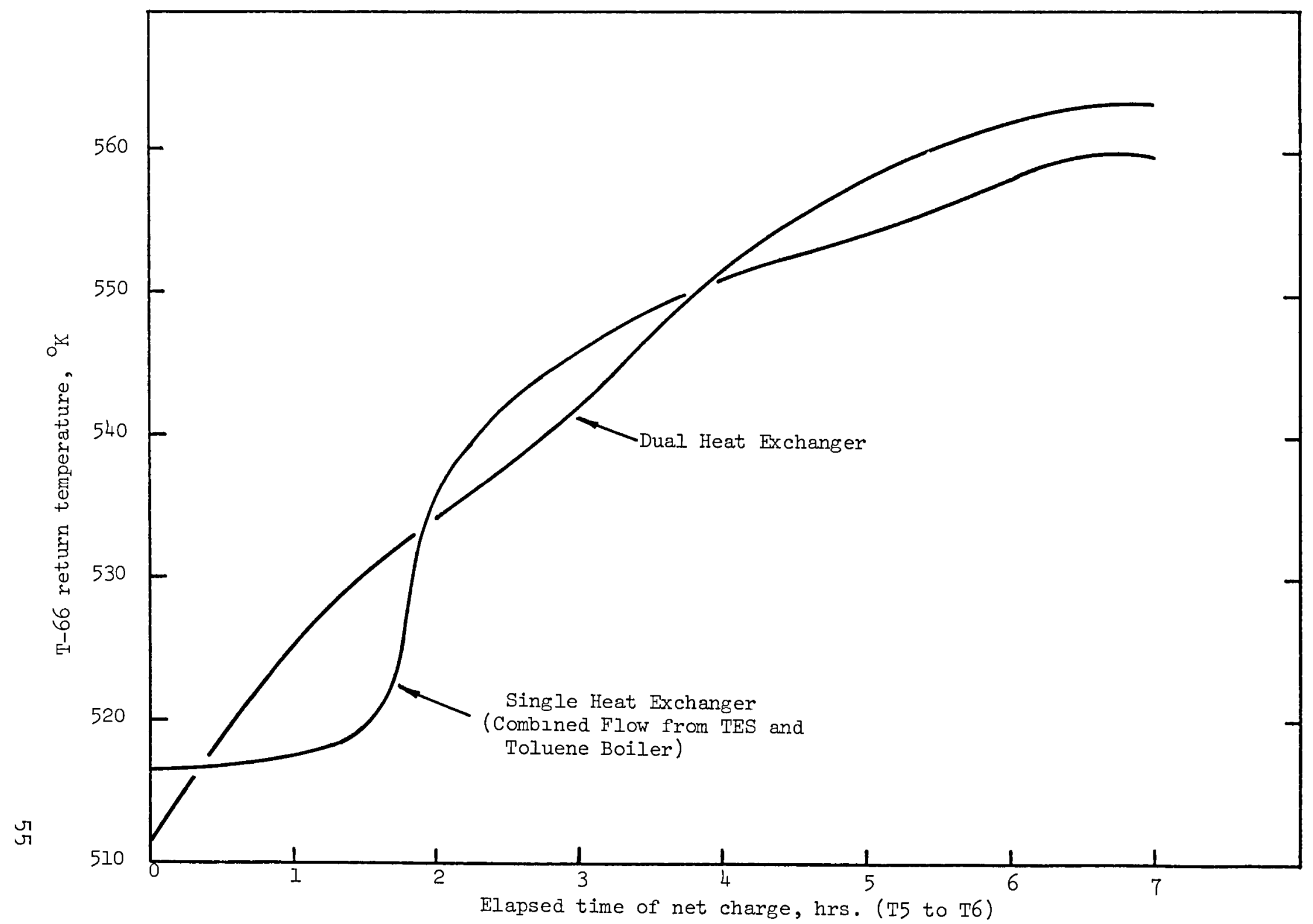

Figure 10. Heat transfer fluid return temperature vs. charge time. 
Output costs incorporate fabrication factors based on weight, surface area, and the "six-tenths rule" whereby fabrication costs increase as the 0.6 power of the size ratio. These methods are generally accepted for making approximate estimates of costs of industrial equipment such as heat exchangers and vessels.

Graphs were made of the parameters against specific cost. Figure 11 shows the FOM against specific cost for several heat exchanger surface areas. The decreasing FOM seen in the $37.2 \mathrm{~m}^{2}\left(400 \mathrm{ft}^{2}\right)$ and $55.7 \mathrm{~m}^{2}\left(600 \mathrm{ft}^{2}\right)$ lines represent smaller Thermkeep masses, and these lines end when a smaller mass no longer produces quality 1.0 vapor.

Figure 12 shows the FOM against specific cost at constant Thermkeep masses. Here there is great improvement in FOM for relatively small cost increases. The reIationships between the two graphs are plotted in Figures 13 and 14 where the surface area and Thermkeep mass are varied at constant FOM. The Reference Design point for the single heat exchanger TESS is also shown on these figures.

The choice of the Reference Design point is somewhat arbitrary, its choice depending upon the relative importance of specific cost and FOM. Figure 11 shows that an FOM as high as .99 can be achieved at a specific cost below that of the single heat exchanger TESS. Therefore -- somewhat arbitrarily -- a Reference Design which betters the single heat exchanger TESS in both specific cost and performance (FOM) is chosen at $55.7 \mathrm{~m}^{2}$ ( $600 \mathrm{ft}^{2}$ ) of each heat exchanger, $13 \mathrm{Mg}$ of Thermkeep, and .95 FOM. It should be noted that, while the absolute specific costs are subject to the limitations noted above, the relative specific costs and performances are realistic. The characteristics of the Reference Designs are shown in Table V.

The specific costs shown on the figures are computed on the same basis for the single, and dual, heat exchanger designs. However, as noted above, the dual heat exchanger eliminates the need for an external toluene boiler. A credit for the boiler was estimated as follows. A communication from the Sandia Laboratories at Albuquerque (Ref. 3) showed that the cost of the boiler in the solar total energy system which the TESS is designed to match, cost $\$ 17,000$ in 1974-1975, and would cost approximately $\$ 25,000$ to replace in 1980 . 


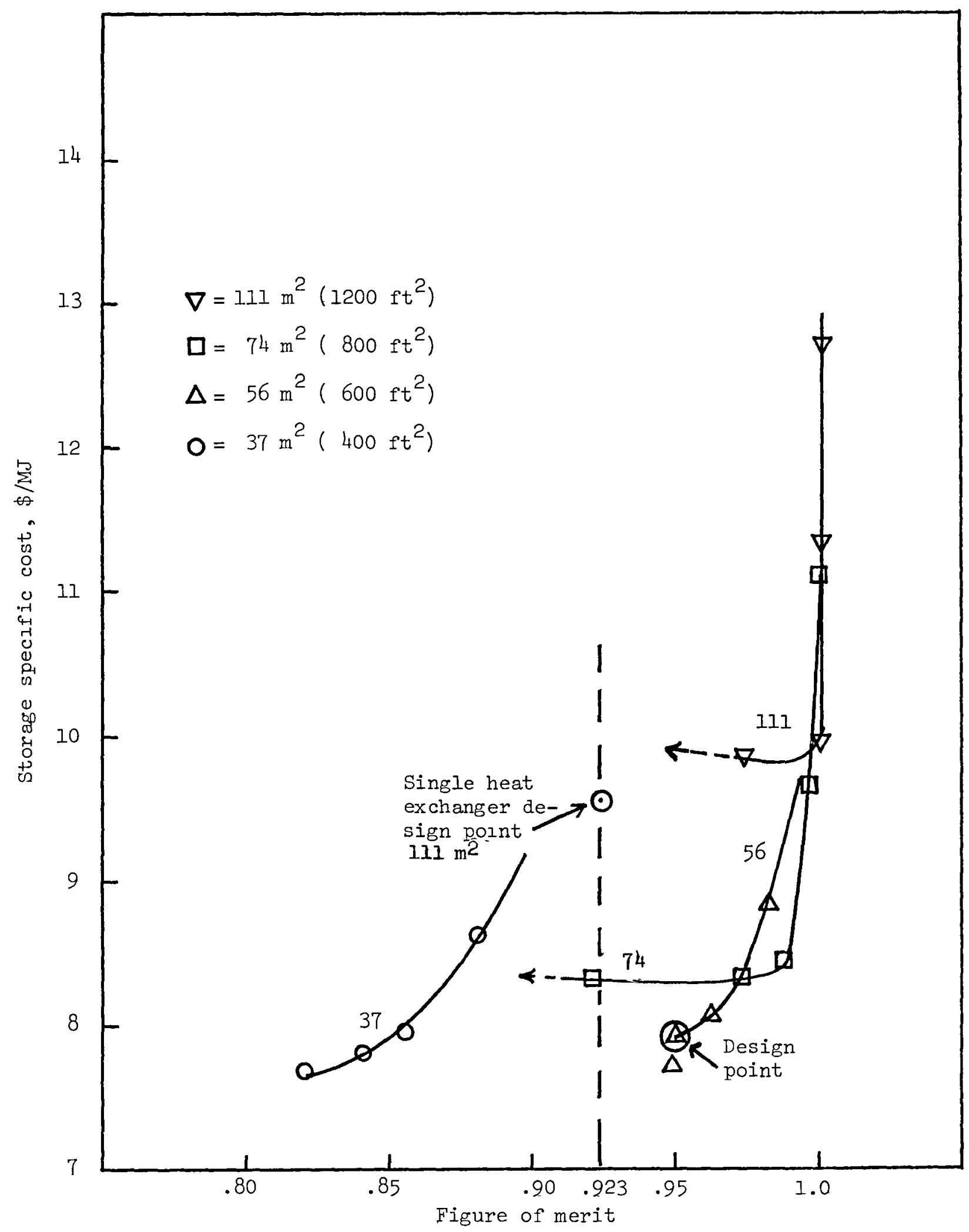

Figure 1l. Specıfic cost vs. Figure of Merlt for varıous heat exchanger areas. 


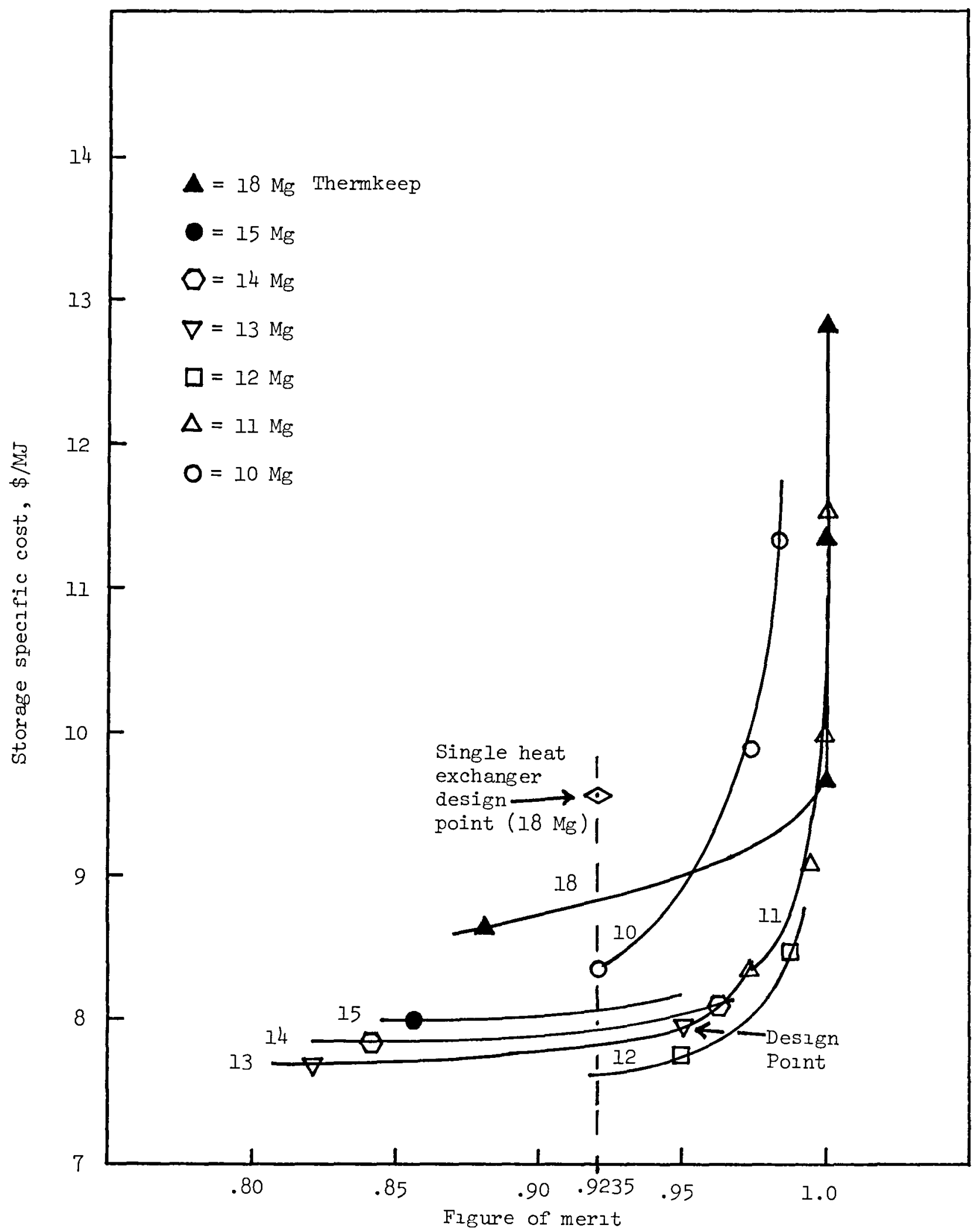

Figure 12. Speciflc cost vs. Figure of Merlt for varıous Thermkeep masses. 


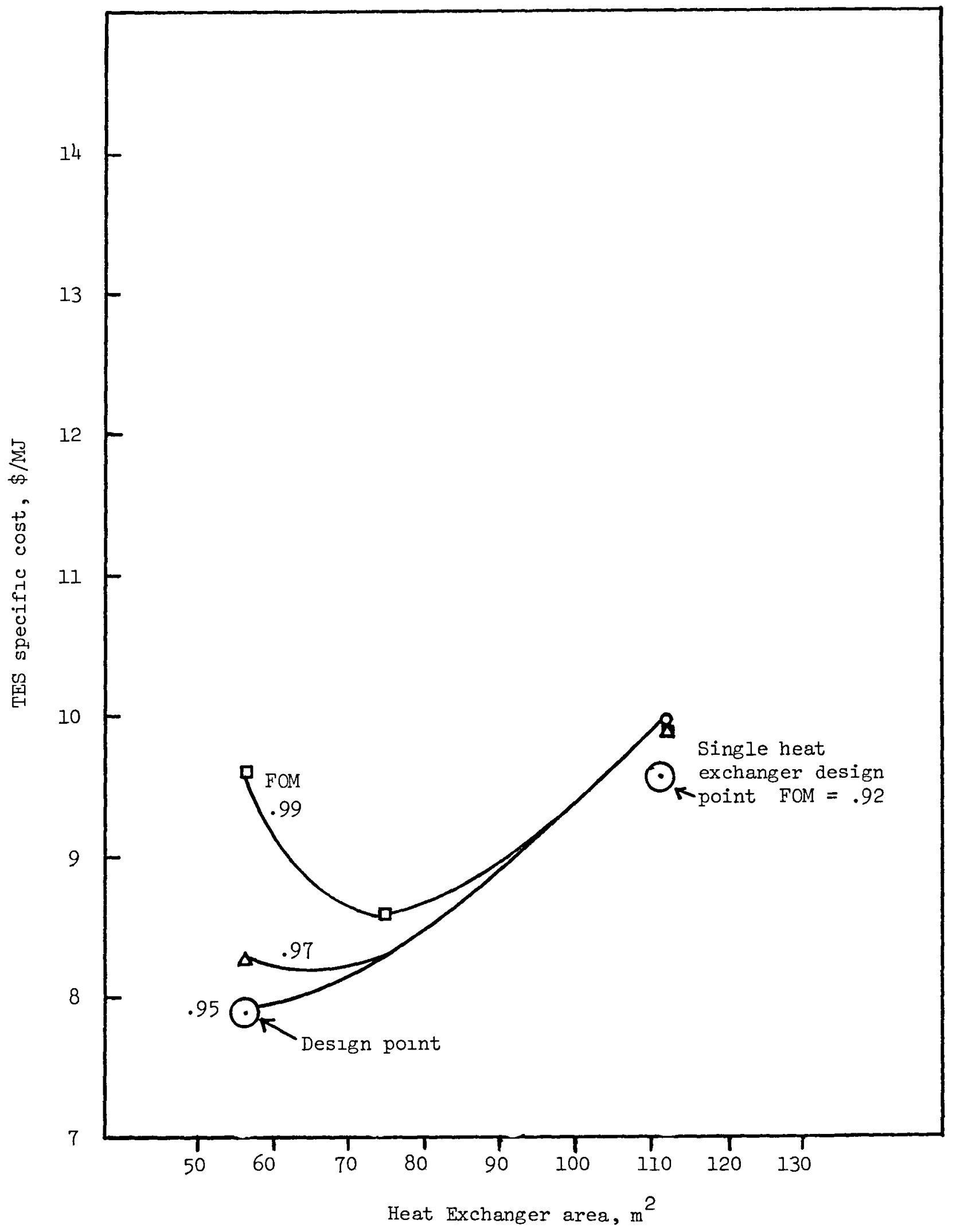

Figure 13. Specific cost vs. heat exchanger area for various Figures of Merit 


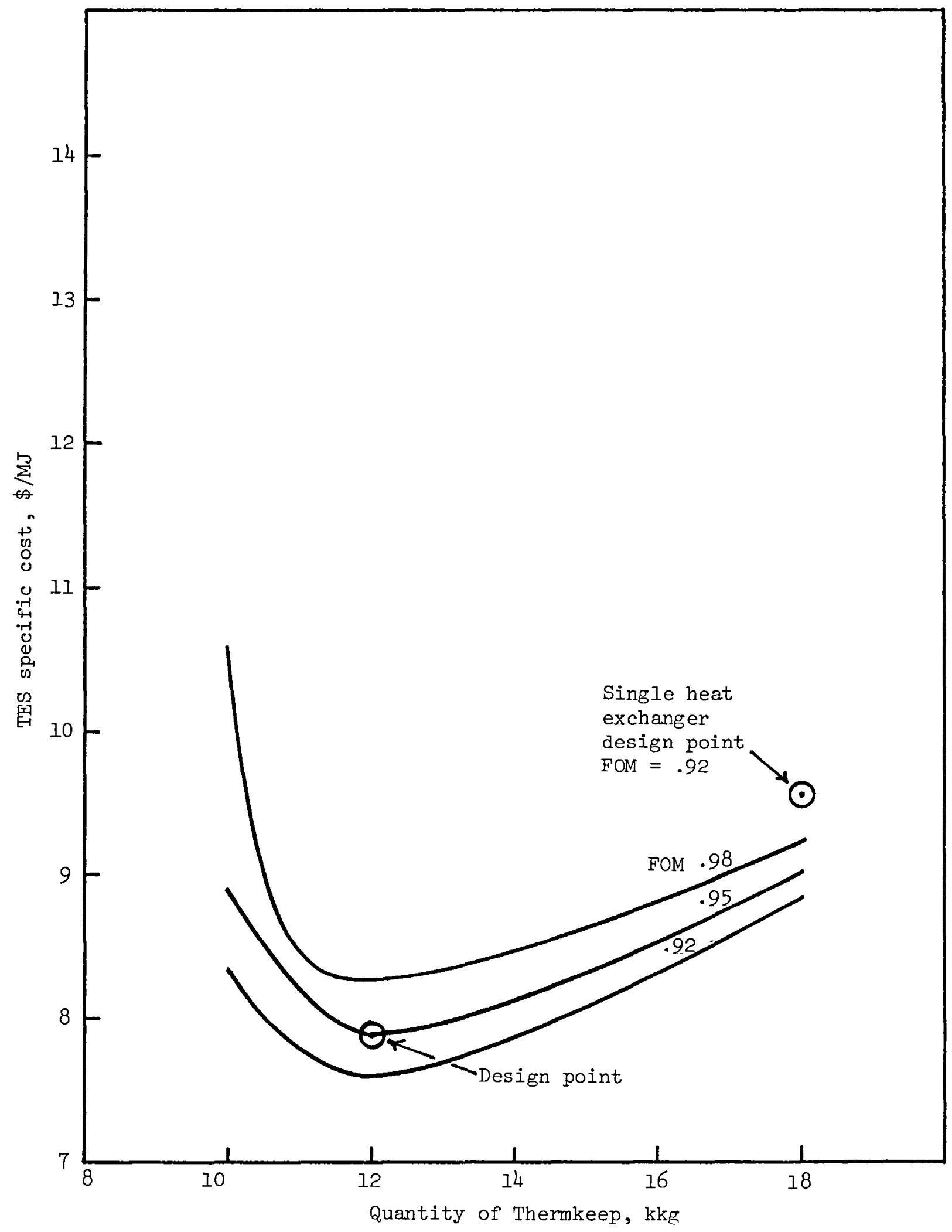

Figure 14. Specific cost vs. Thermkeep mass for various Figures of Merıt. 
Cost Calculation Input Data

Shroud material cost, $\$ / \mathrm{kg}$

Shroud side fabrication cost, $\$ / \mathrm{m}^{2}$

Shroud side fabrication cost, $\$ / \mathrm{kg}$

Shroud top fabrication cost, $\$ / \mathrm{m}^{2}$

Shroud top fabrication cost, $\$ / \mathrm{kg}$

Shroud bottom fabrication cost, $\$ / \mathrm{m}^{2}$

Shroud bottom fabrication cost, $\$ / \mathrm{kg}$

Shroud top thlckness, m

Shroud side thickness, $m$

Shroud bottom thickness, $m$

Vessel wall material cost, $\$ / \mathrm{kg}$

Vessel end material cost, $\$ / \mathrm{kg}$

Vessel fabrication cost, $\$ / \mathrm{m}^{2}$

Vessel fabrication cost, $\$ / \mathrm{kg}$

Tube material cost coefficient

Tube material cost, $\$ / \mathrm{kg}$

Tube fabrication cost, $\$ / \mathrm{kg}$

Tube fabrication cost, $\$ / \mathrm{m}^{2}$

Storage material cost, $\$ / \mathrm{kg}$

Insulation cost, $\$ / \mathrm{kg}$

Cost Calculation Output Data

Tube material cost, $\$$

Total tube cost, $\$$

Storage material cost, $\$$

Insulation cost, \$

Shroud side material cost, \$

Shroud side total cost, \$

Shroud bottom material cost, $\$$

Shroud bottom total cost, \$

Shroud top material cost, \$

Shroud top total cost, $\$$

Shroud total material cost, $\$$

Shroud total cost, \$

Vessel material cost, \$

Vessel total cost, $\$$

Total materials cost for TES, $\$$

Total fabrication cost for TES, $\$$

TES total cost, $\$$

\% OF TOTAL COST, HFAT EXCHANGER
Dual Heat

Exchanger

Design Point
Single Heat

Exchanger

Comparison Point
.509

10.14

1.038

77.35

7.92

99.84

7.67
$1.524 \times 10^{-3}$

$1.2192 \times 10^{-3}$

$3.4036 \times 10^{-3}$

.4718

.4806

615.47

2.447

.028

3.9091

11.9

964.3398

.441

.2866
.509

10.14

1.038

77.35

7.92

99.84

7.67

$1.524 \times 10^{-3}$

1. $2192 \times 10^{-3}$

$3.4036 \times 10^{-3}$

.4718

.4806

615.47

2.447

.028

5.1998

11.9

964.3398

.441

.2866
1479.

14331.

5733.

992.

173.

336.

120.

582.

54.

315.

346.

1232.

1321.

3271.

9872.

15687.

25559.

56
2013. 15190 .

7938.

1173.

199.

376.

138.

640 .

62.

345.

398.

1361.

1644.

3929.

13166.

16426.

29592.

51 
This represents an annual cost increase over the six years of $6.6 \%$. On this basis the cost in 1977, the year in which TESS comparative costs are based, would have been $\$ 20,600$. This was subtracted from the computed TESS cost in order to obtain a Storage Specific Cost After Boiler Credit.

An additional benefit would accrue to the dual heat exchanger TESS in the Sandia solar total energy system resulting from the elimination toluene boiler; namely, the heat loss from the boiler during the nighttime system shutdown, especially in winter (Ref. 4). Since this represents an operating cost benefit, no credit is taken in the capital costs reported here.

The side-by-side tube concept was chosen for this study because it is simple to represent mathematically, and a method of constructing such tubing can be easily visualized. For small diameters $(.004-.03 \mathrm{~m})$, the cost per unit weight of tubing decreases as diameter increases and this is reflected in the heat exchanger costs of the Reference Design. A double tube consisting of two identical tubes was considered to have a material cost just twice that of a single tube. These factors may unfairly favor the dual tube costs since they are based upon a tubing configuration which is not known to be readily available. However, it seems probable that such tubing can readily be manufactured. For example, two tubes might be passed side-by-side through a continuous MIG (metal-inert gas) welding machine which lays a fillet between them, or an electric current could be applied across them to cause a continuous line weld between them.

Another concept which might apply would use two coaxial tubes with an annulus between, plus a single tube. One fluid would flow through the annulus, while the other fluid would flow through the inner coaxial tube and through the single tube. This system provides for direct heat exchange between the two fluids, and between each fluid and the storage medium. Coaxial tube configurations are presently available; designs exist with fins and wire in the annulus to promote turbulence or direct heat transfer between the inner and outer walls.

The computation of the single and dual heat exchanger costs on the same material and fabrication cost basis may underestimate the cost of the dual heat exchanger. In order to show the latitude for cost increase, the cost of the dual heat exchanger was modified in the following three cases: 
Case I

The material cost of tubing of the dual heat exchanger was fixed at twice that of the single tube exchanger ( $\$ 10.4 / \mathrm{kg}$ vs. $\$ 5.2 / \mathrm{kg}$ ) to eliminate the cost advantage of larger diameters. Then the fabrication costs were allowed to increase $22 \%$ until the storage specific cost of the dual heat exchanger TESS was the same as that of the single tube heat exchanger TESS, viz. \$2.57/mJ.

Case 2

The material cost was kept the same $(\$ 3.9 / \mathrm{kg})$, and it was found that the fabrication costs could be allowed to increase $41 \%$ before the storage specific cost of the single tube heat exchanger TESS was exceeded.

Case 3

The material cost was kept the same but the fabrication costs were doubled. This produced a storage specific cost of $\$ 11.91 / \mathrm{mJ}$ for the dual heat exchanger TESS.

The results of these comparisons are shown in Table VI.

Table VII shows the percentage of total cost of the three cases of the dual heat exchanger TESS described above, and for the single heat exchanger comparison case.

The characteristics of the Reference Designs for the dual and single heat exchanger are summarized on Table VIII where the Case 3 heat exchanger cost has been assumed for the dual heat exchanger TESS in order to present the results on what is believed to be a conservative basis. On this basis the dual heat exchanger TESS has a specific cost which is higher than the single heat exchanger TESS by $25 \%$ before the boiler credit, but which is only $58 \%$ of the single heat exchanger TESS cost after boiler credit. This cost saving is achieved while the performance as measured by the Figure of Merit is improved from .92 to .95 . 


\section{TABLE VI}

COMPARISON OF THREE METHODS OF CALCULATING

HEAT EXCHANGER COST FOR DUAL HEAT EXCHANGER TESS

\section{Case 1 Case 2 Case 3}

Cost Calculation Input Data:

Tube Material Cost Coefficient Tube Material Cost, $\$ / \mathrm{kg}$

Tube Fabrication Cost, $\$ / \mathrm{kg}$

Tube Fabrication Cost $\$ / \mathrm{m}^{2}$

$\begin{array}{lll}.0745 & .028 & .028 \\ 10.401 & 3.909 & 3.909 \\ 14.518 & 16.815 & 23.8 \\ 1176 & 1362 & 1929\end{array}$

Cost Calculation Output:

Tube Material Cost, $\$$

Total Tube Cost, \$

Total TESS Materials Cost \$

Total TESS Fabrication Cost \$

Total TESS Cost \$

3934

19614

12327

18515

30842

Storage Specific Cost, \$/MJ:

Before boiler credit

After boiler credit

$\begin{array}{lll}9.57 & 9.57 & 11.91 \\ 3.18 & 3.18 & 5.52\end{array}$


TABLE VII

PER CENT OF TOTAL COST OF TESS

Dual Heat Exchanger

\section{Case 1 and Case 2 Case 3}

Thermkeep

Heat exchanger

Containment

Thermal insulation

Outer shroud

19

15

64

11

3

4
Single

Heat

Exchanger

27

51

13

4

5 


\begin{tabular}{lll} 
& \multicolumn{1}{c}{ Dual } & Single \\
\cline { 2 - 2 } Tank height and diameter, & 2.11 & 2.35 \\
System helght and diameter, m & 3.4 & 3.6 \\
Quantity of Thermkeep, kg & 13,000 & 18,000 \\
Heat exchanger: & & \\
Tube inside diameter, cm & .716 & \\
Number of tubes & $150(\mathrm{prs})$ & 280 \\
Total length, m & 5588 & 7761 \\
Figure of merit & .95 & .92 \\
Total cost $\$$ & 38411 & 29592 \\
Speclfic cost, $\$ / M J$ & 11.91 & 9.57 \\
Boiler credit $\$$ & 20600 & None \\
Net specific cost, $\$ / M J$ & 5.52 & 9.57
\end{tabular}




\section{CONCLUSIONS}

A program of experimentation and analysis has been carried out to continue and extend the work reported in NASA CR-159465, on the development of a phasechange thermal energy storage system (TESS) using modified anhydrous sodium hydroxide for solar electric power generation.

The phase-change medium used comprises commercial grade anhydrous $\mathrm{NaOH}$ modified by the addition of $8 \%$ (wt) of $\mathrm{NaNO}_{3}$ and $0.2 \% \mathrm{MnO}_{2}$.

The TESS studied in the prior work utilizes a single heat exchanger and a non-phase-change heat transfer fluid, and is designed to meet the following operating conditions :

Storage capacity is $3.1 \times 10^{6} \mathrm{~kJ}$ operating over the range $516 \mathrm{~K}$ to $584 \mathrm{~K}$. Heat is charged into the storage medium at a maximum rate of $1.8 \times 10^{6} \mathrm{~kJ} / \mathrm{hr}$, at a temperature of $584 \mathrm{~K}+2 \mathrm{~K}$. Heat is discharged from storage at a maximum rate of $1.0 \times 10^{6}$ $\mathrm{kJ} / \mathrm{hr}$ at a temperature of $582 \mathrm{~K} \pm 2 \mathrm{~K}$.

The present program consisted of three parts:

Part I extends the prior work by means of chemical analyses of the storage medium, and examination of the heat exchanger of the one-tenth scale experimental model constructed and tested in the prior program.

Part II utilized the physical model of the prior program to obtain experimental data with a different heat transfer fluid, Caloria HT-43, for further verification of a computer model of the prior program which used Therminol-66.

Part III included the development of a new computer model which introduced a second heat exchanger into the TESS for evaporating and superheating the power fluid for a Rankine cycle power generator. This was used to produce a cost optimize reference design of a TESS for comparison with the single heat exchanger design of the prior work. 
No chemical deterioration of the medium was found. Some physical segregation of components was observed, which is attributed to the fact that the medium is a noneutectic mixture, but no corresponding deterioration in performance was noted. Physical segregation can be reversed by periodic complete melting of the medium.

No significant physical deterioration of the heat exchanger was observed.

The dual heat exchanger TESS was found to be superior to the single heat exchanger design in performance and in capital cost, due to savings resulting from the elimination of an external heat exchanger. Additional advantages result from system operating cost savings.

Auxiliary heating is used to raise the temperature of the power fluid vapor to the specified temperature, and a figure of merit is defined as the ratio of the heat from storage to the sum of the heat from storage plus the auxiliary heat. This is used as the measure of performance.

The design comparison is summarized as follows:

Dual Heat Single Heat

Exchangers Exchangers

Quantity of storage medium, kg

13,000

18,000

Heat exchanger tubing

Inside diameter, $\mathrm{cm}$

Total length, $\mathrm{m}$

0.716

5588

0.457

$\$ 38,411$

7761

Total cost

11.91

$\$ 29,592$

Specific cost, \$/MJ

$\$ 20,600$

9.57

Boiler credit

5.52

$-0-$

Net specific cost, \$/MJ

9.57

Figure of Merit

.95

.92 


$$
\text { A }
$$

\section{THERMKEEP POST TEST ANALYSIS}

(Technical Report - Skinner \& Sherman, Inc. 



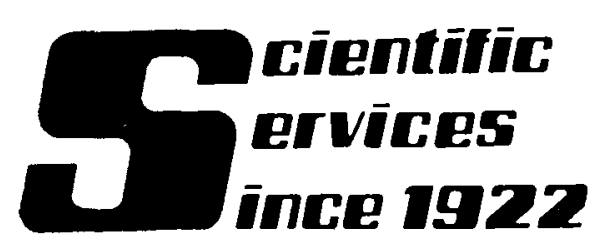

CHEMICAL a PHYSICAL

ELECTRICAL - BACTERIOLOGICAL

\section{TECHNICAL REPORT prepared for}

COMSTOCK \& WESCOTT, INC.

CASE NO. 11833 

CIIENT: Comstock \& Wescott, Inc. 765 Concord Avenue Cambridge, NA 02138

Attention: Mr. Richard Rice

CASE NO:

11833

REFERENCE: Purchase Order No. 29519

PROJECT DESCRIPTION:

To analyze fifteen (15) samples of inorganic salt mixture for $\mathrm{NaNO}_{3}$, $\mathrm{NaNO}_{2}, \mathrm{MnO}_{2}, \mathrm{Na}_{2} \mathrm{CO}_{3}$ and $\mathrm{NaCl}$.

\section{SAYPLE IDENTIFICATION:}

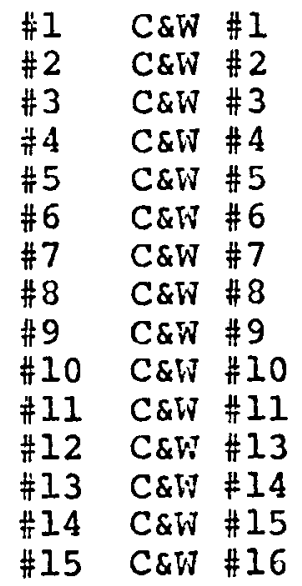

METHODS OF TESTS:

1. Standard Methods for the Examination of Water and Wastewater, 14th Edition, 1975.

2. Atomic Absorption Spectroscopy for total manganese.

This report is rendered upon all of the following conditions This report is not to be reproduced wholly or in part without special permission in writing Total hability is limited to the invoiced amoun Skınner \& Sherman Inc Skinner \& Sherman Laboratories Inc and/or New England Laboratories name and/or logos may not be used in conjunction with the contents of this report in any advertising media The results listed refer only to tested samples and applicable parameters Product endorsement is in conjunction with the contents of this report in any advertising media The results listed refer only to tested samples and applicable parameters Product endorsement is
neither inferred nor implied Samples are held for thirty days following issuance of report Samples will be stored at client's expense if authorized in writing

\section{skïnngr Es sherman laboratories ins.}


CIIENT: $\quad$ Comstock \& Tescott, Inc.

CASE NO: $\quad 11833$

3. Colorimetric Method, using spectrophotometer, Beckman DB for sodium nitrite.

4. Official Methods of Analysis of the Association of Official Analytical Chemists.

5. Scott's Standard Methods of Chemical Analysis.

RESULTS OF ANALYSIS:

\begin{tabular}{|c|c|c|c|c|}
\hline & $\begin{array}{l}\text { Sodium } \\
\text { Nitrite }\end{array}$ & $\begin{array}{l}\text { Sodium } \\
\text { Carbonate }\end{array}$ & $\begin{array}{l}\text { Sodium } \\
\text { Chloride }\end{array}$ & $\begin{array}{r}\text { Man } \\
\text { Di }\end{array}$ \\
\hline
\end{tabular}

$\begin{array}{llllll}\text { Sample \#1 } & 3.467 & 0.0306 & 2.62 & 2.03 & 0.172 \\ \text { Sample \#2 } & 7.477 & 0.0307 & 1.45 & 1.98 & 0.171 \\ \text { Sample \#3 } & 4.652 & 0.0020 & 2.85 & 1.73 & 0.101 \\ \text { Sample \#4 } & 5.278 & 0.0021 & 1.48 & 1.35 & 0.085 \\ \text { Sample \#5 } & 5.041 & 0.0023 & 1.55 & 1.47 & 0.113 \\ \text { Sample \#6 } & 5.326 & 0.0022 & 1.43 & 1.63 & 0.110 \\ \text { Sample \#7 } & 4.503 & 0.0019 & 1.38 & 1.68 & 0.083 \\ \text { Sample \#8 } & 4.377 & 0.0023 & 1.35 & 1.85 & 0.110 \\ \text { Sample \#9 } & 5.623 & 0.0023 & 2.89 & 1.65 & 0.102 \\ \text { Sample \#10 } & 4.878 & 0.0026 & 1.36 & 1.76 & 0.095 \\ \text { Sample \#11 } & 2.791 & 0.0017 & 1.44 & 1.53 & 0.042 \\ \text { Sample \#13 } & 2.707 & 0.0021 & 1.40 & 1.76 & 0.057 \\ \text { Sample \#14 } & 2.546 & 0.0032 & 1.32 & 1.25 & 0.053 \\ \text { Sample \#15 } & 5.772 & 0.0098 & 2.88 & 1.58 & 0.137 \\ \text { Sample \#16 } & 5.138 & 0.0095 & 2.90 & 1.43 & 0.172\end{array}$

Respectfully submitted,

SKINNER \& SHERMAN LABORATORIES, INC.

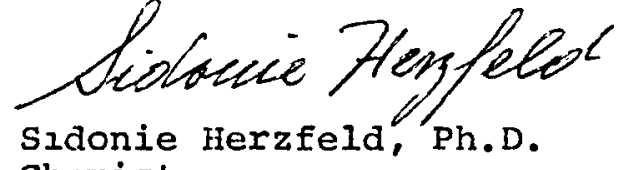

Chemist

$\mathrm{SH} / 1 \mathbf{S}$

This report is rendered upon all of the following conditions This report is not to be reproduced wholly or in part without special permission in writing Total lability is limited to the invoiced amount Skınner \& Sherman, Inc, Skınner \& Sherman Laboratories, Inc and/or New England Laboratories name and/or logos may not be used in conjunction with the contents of this report in any advertising media The results listed refer only to tested samples and applicable parameters Product endorsement is nether inferred nor implied Samples are held for thirty days following issuance of report Samples will be stored at client's expense if authorized in writing A-6 


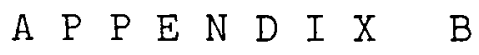

HYSTERESIS STUDY GRAPHS

B-I 


\section{SYMBOLS}

Thermkeep temperatures:

$$
\begin{aligned}
& 0=\begin{array}{l}
\text { Shallow well temperatures vs. } \\
\text { location in tank }
\end{array} \\
& \Delta=\begin{array}{l}
\text { Deep well temperatures vs. } \\
\text { location in tank }
\end{array} \\
& +=\text { Computer model predictions }
\end{aligned}
$$




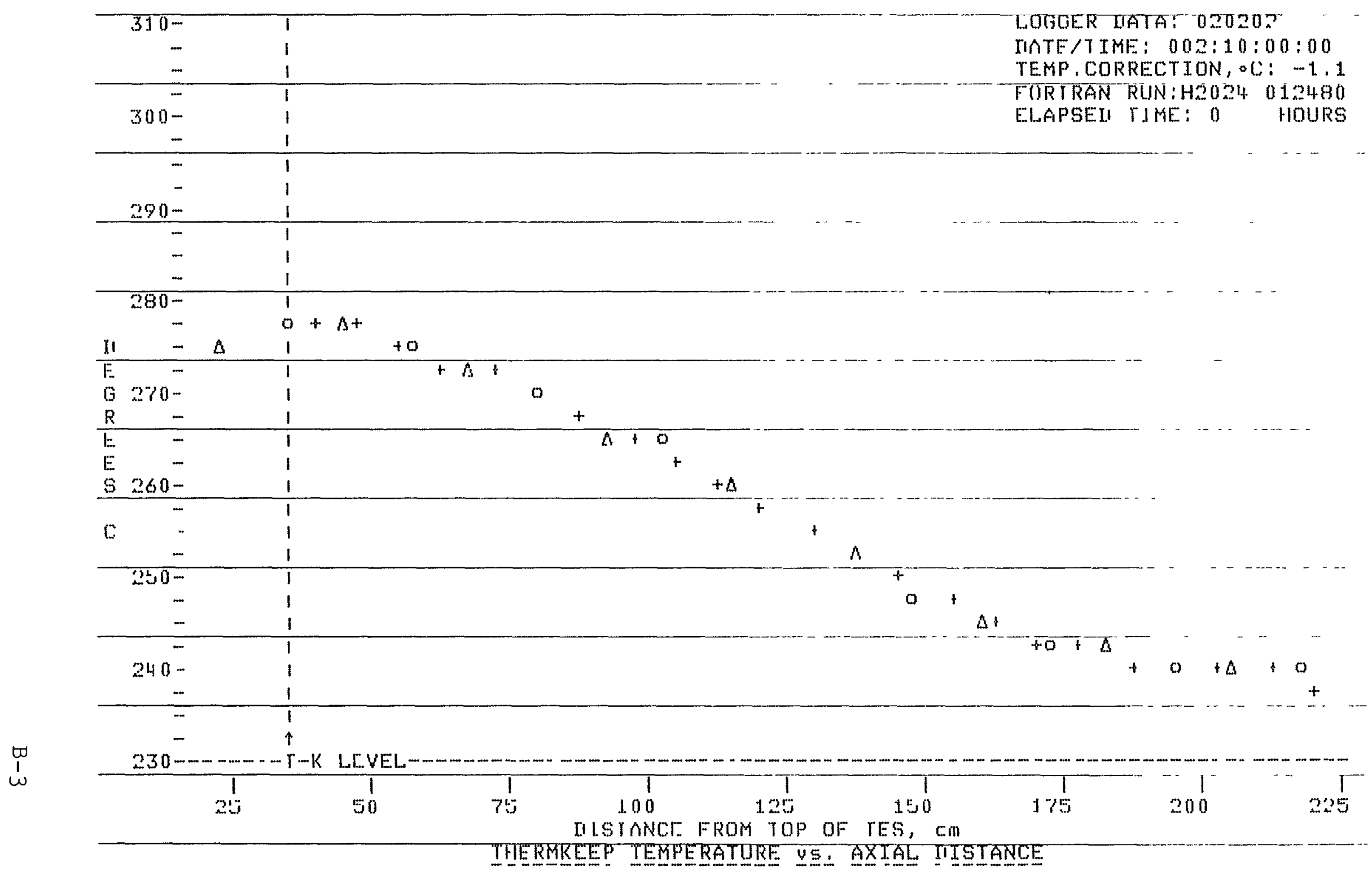

Figure B-1. Test No. I 


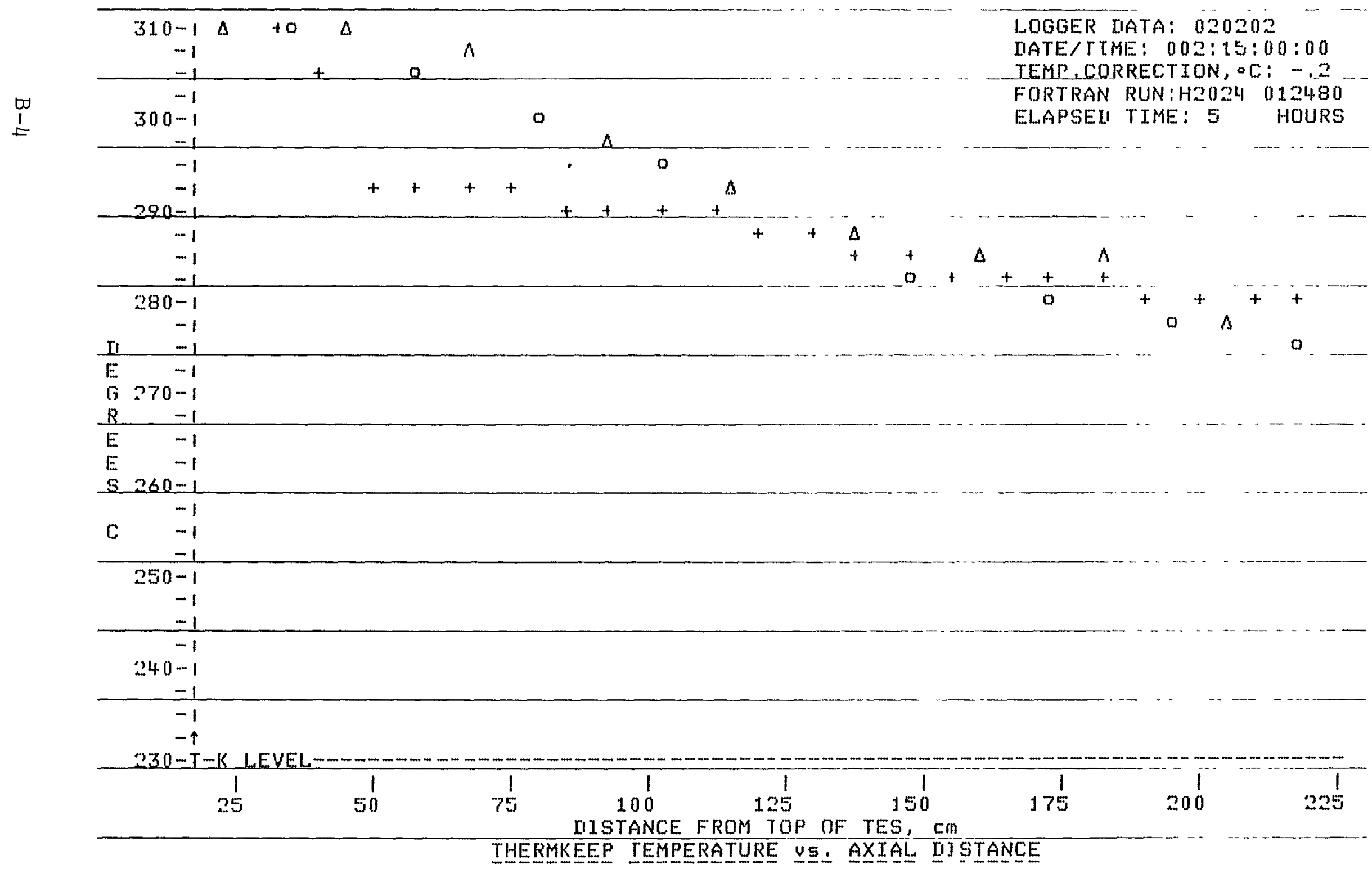

Figure B-2. Test No. I 


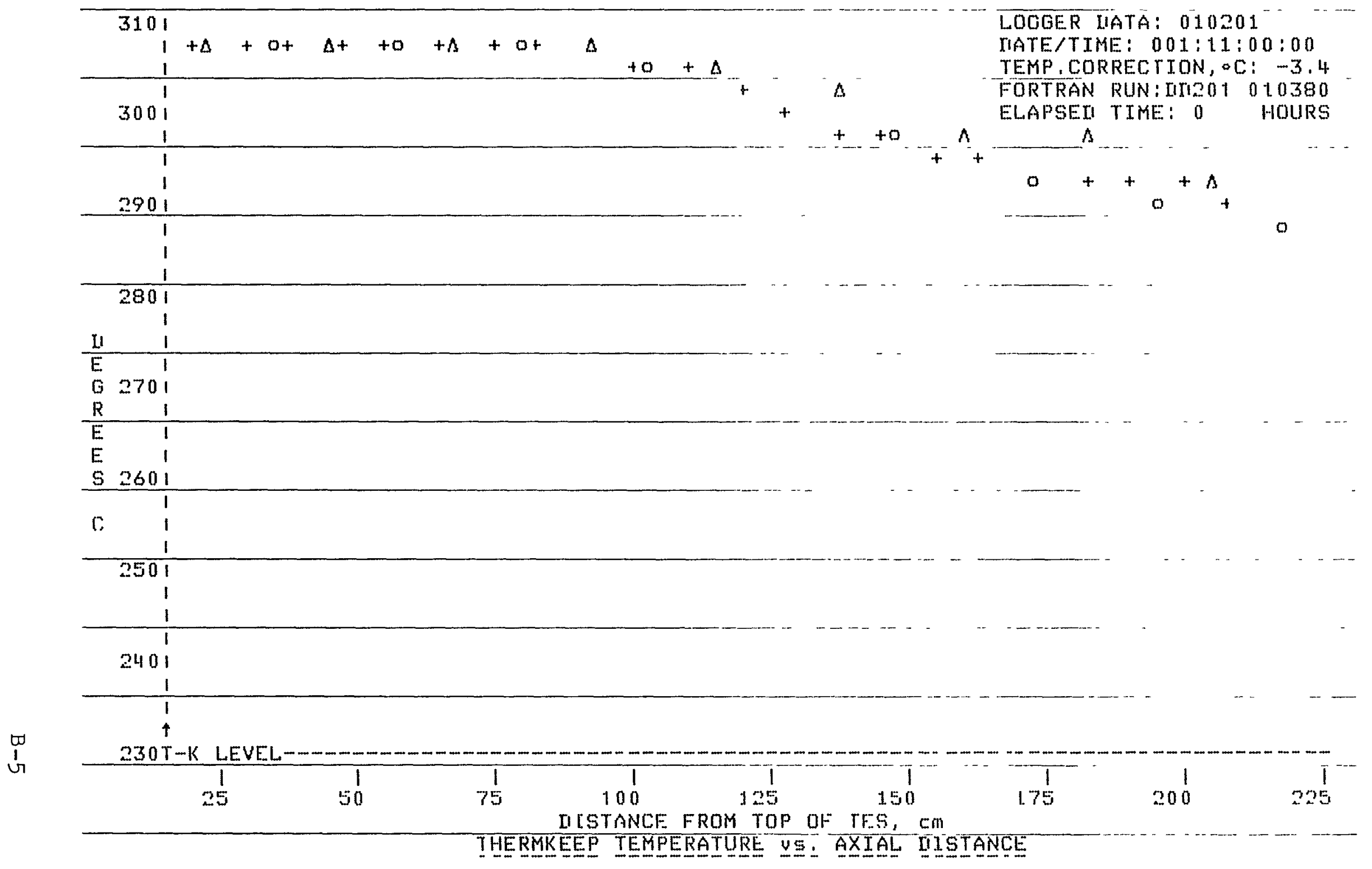

Figure B-3. Test No. 2 


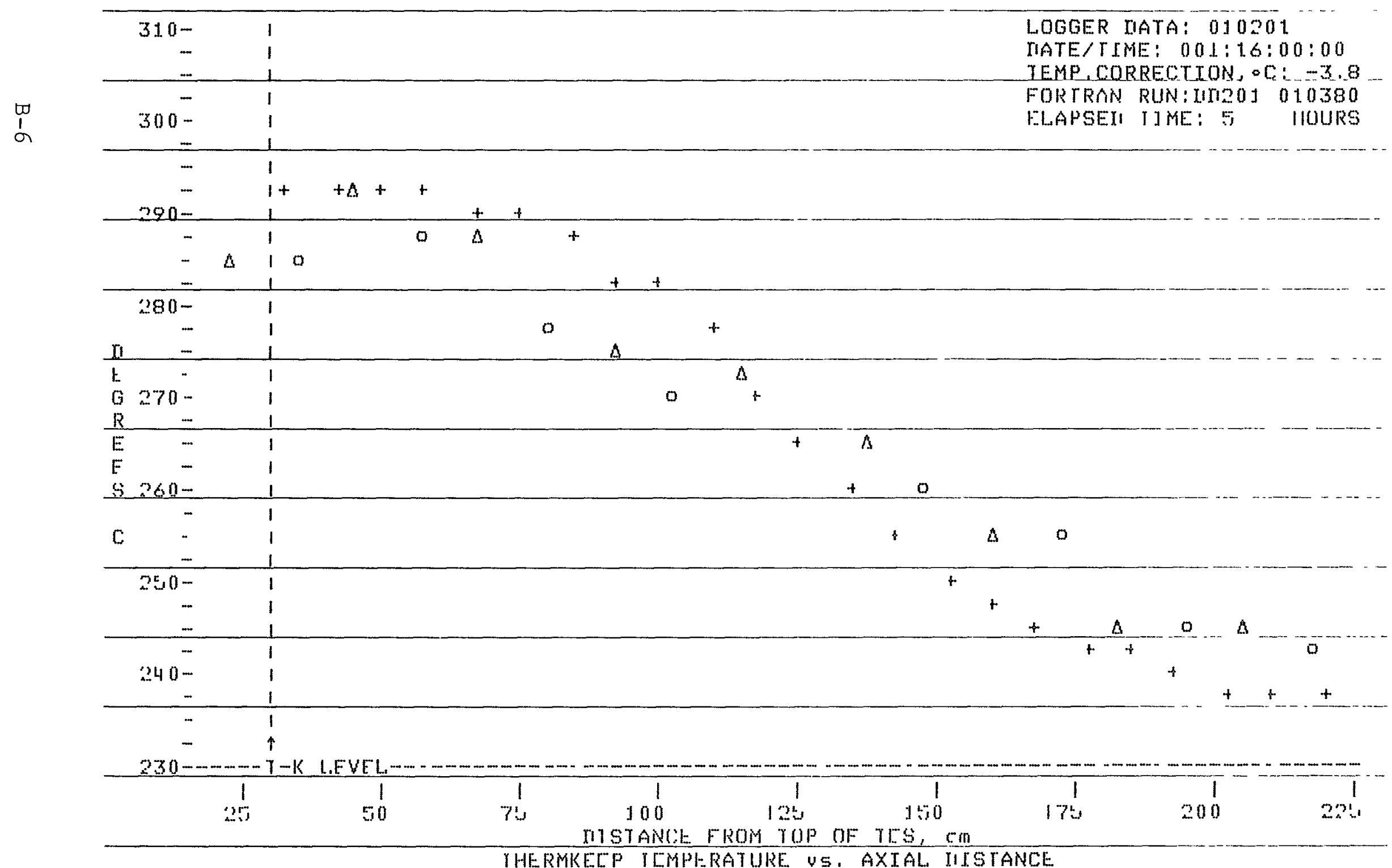

Figure B-4. Test No. 2. 


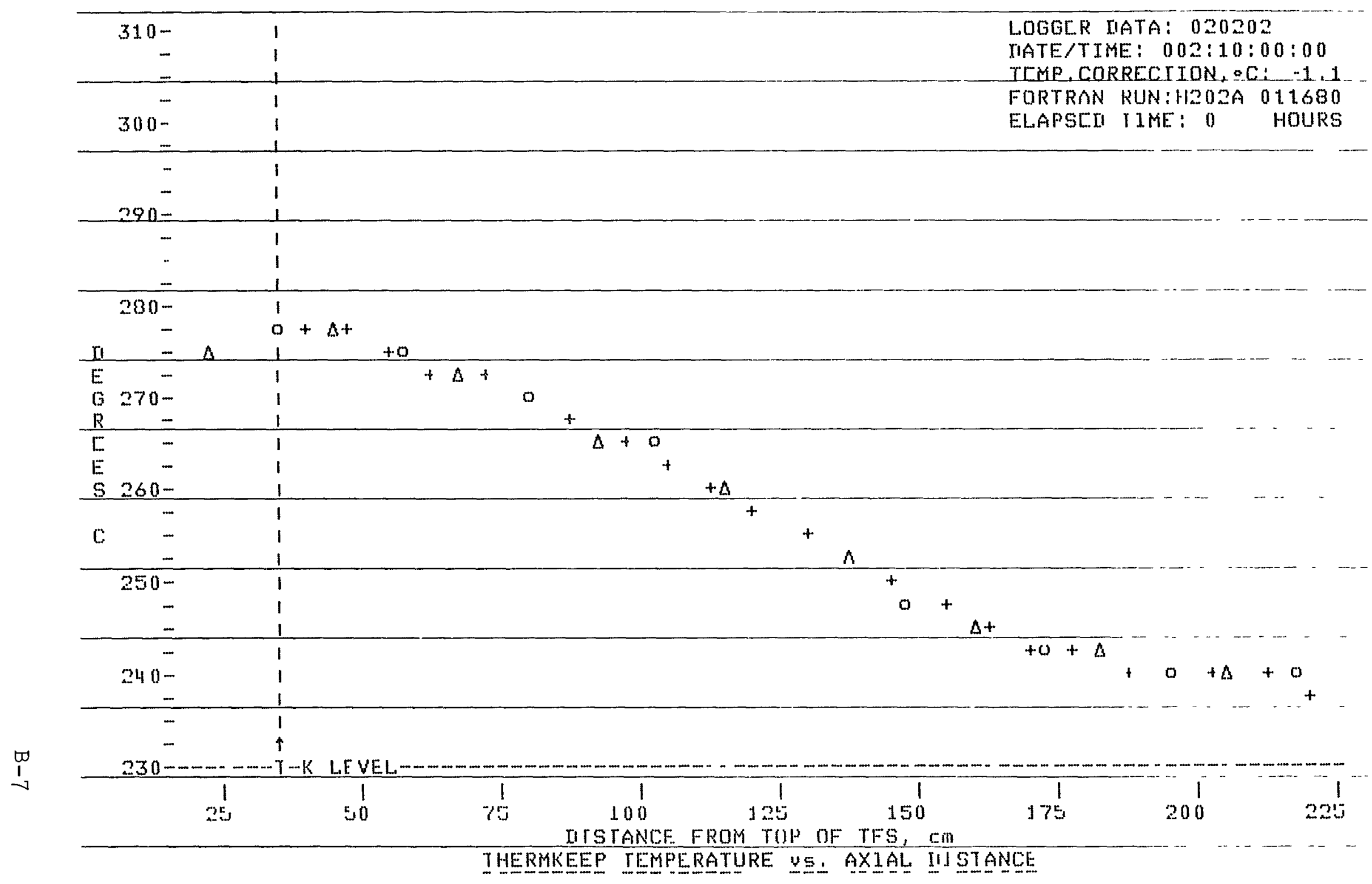

Figure B-5. Test No. 3 


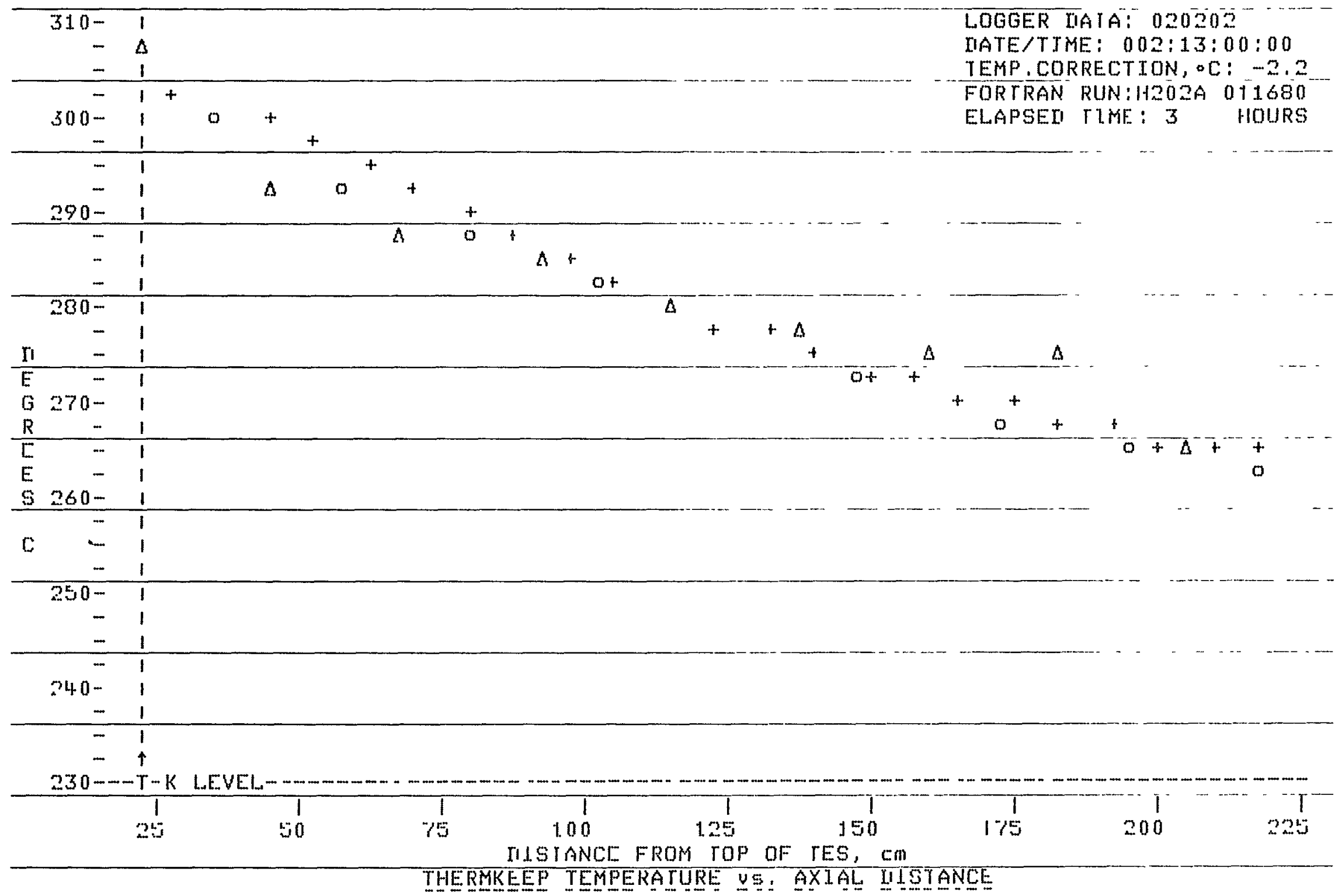

Figure B-6. Test No. 3 


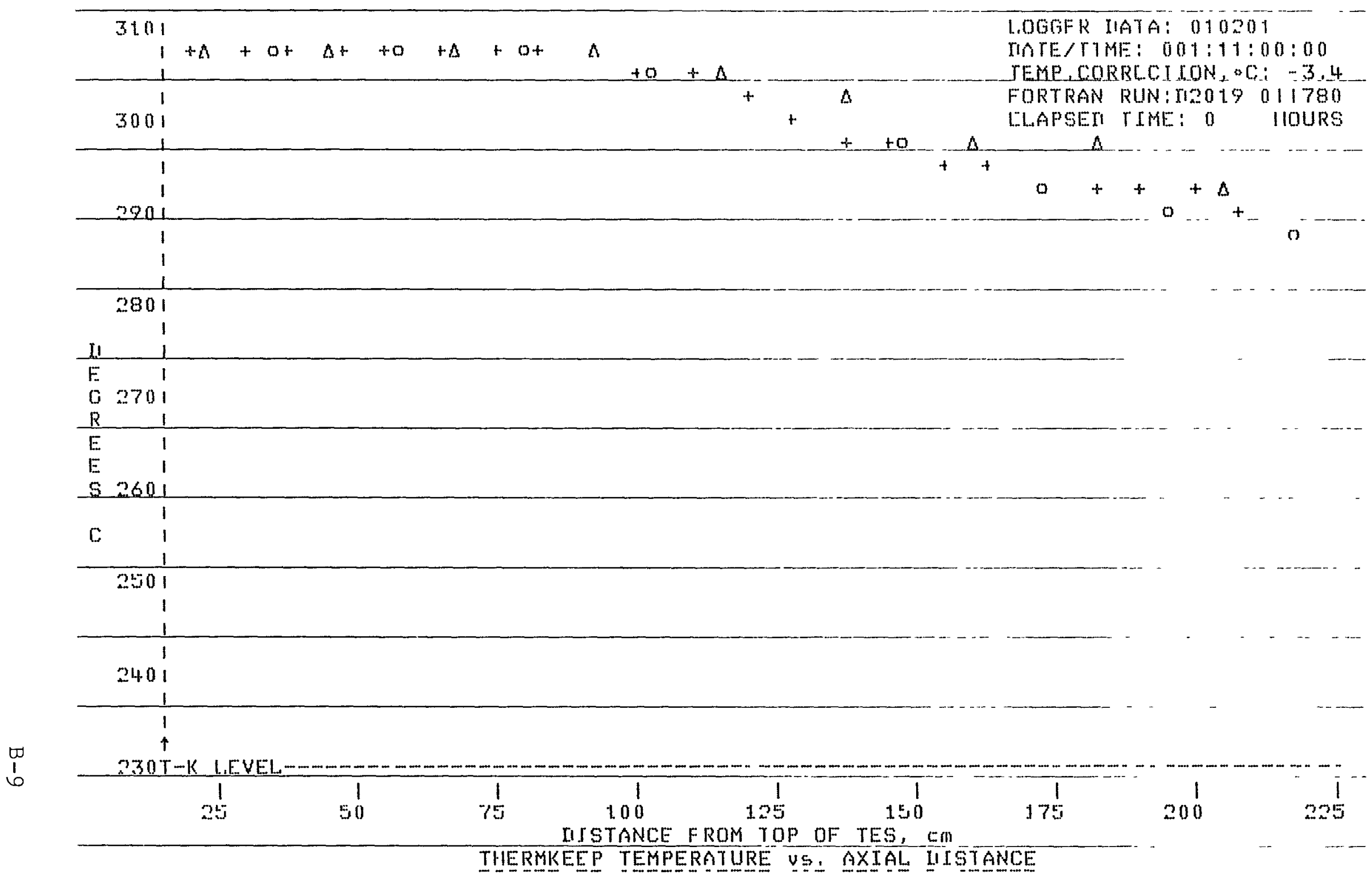

Figure B-7. Test No. 4. 


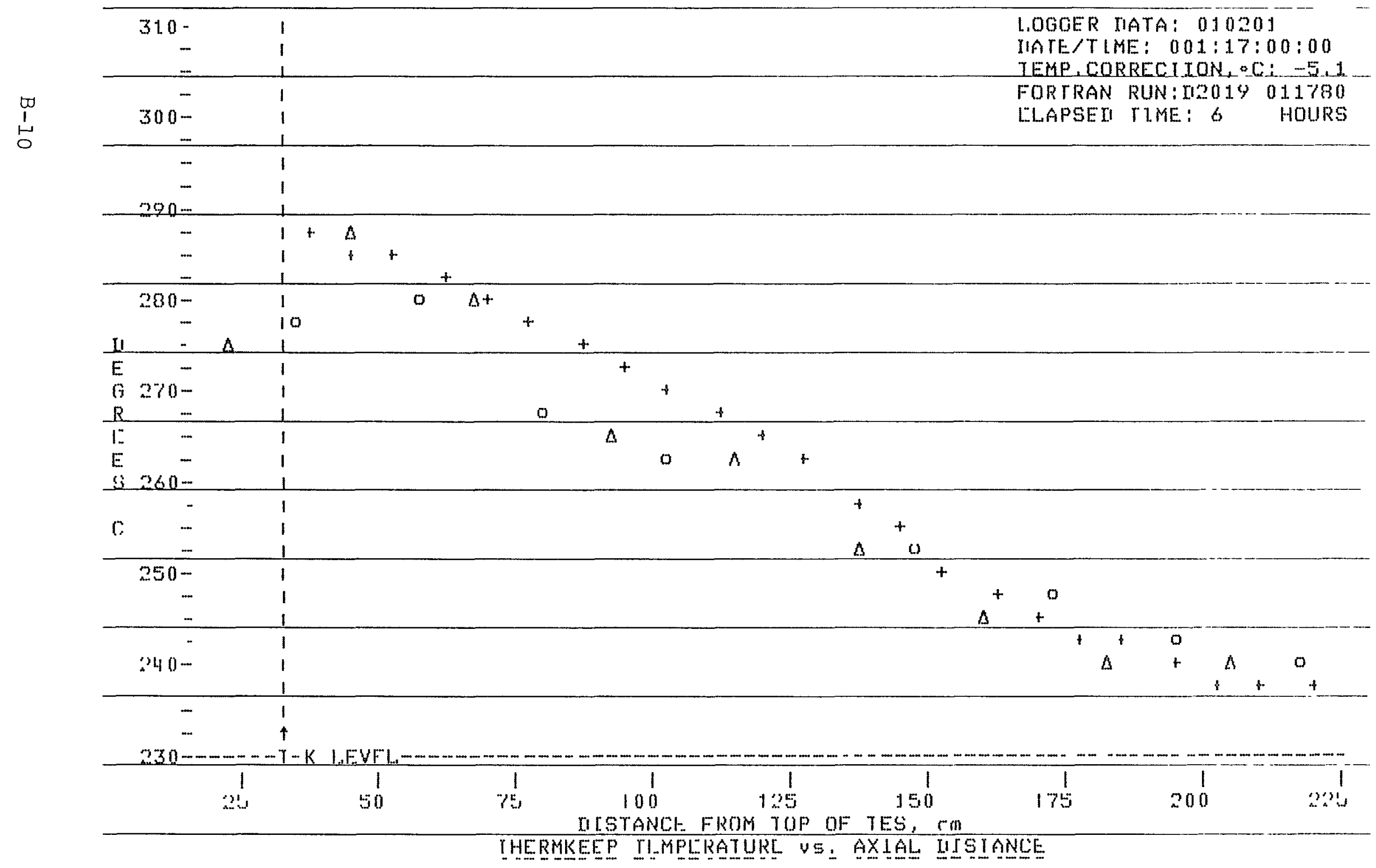

Figure B-8. Test No, 4 . 


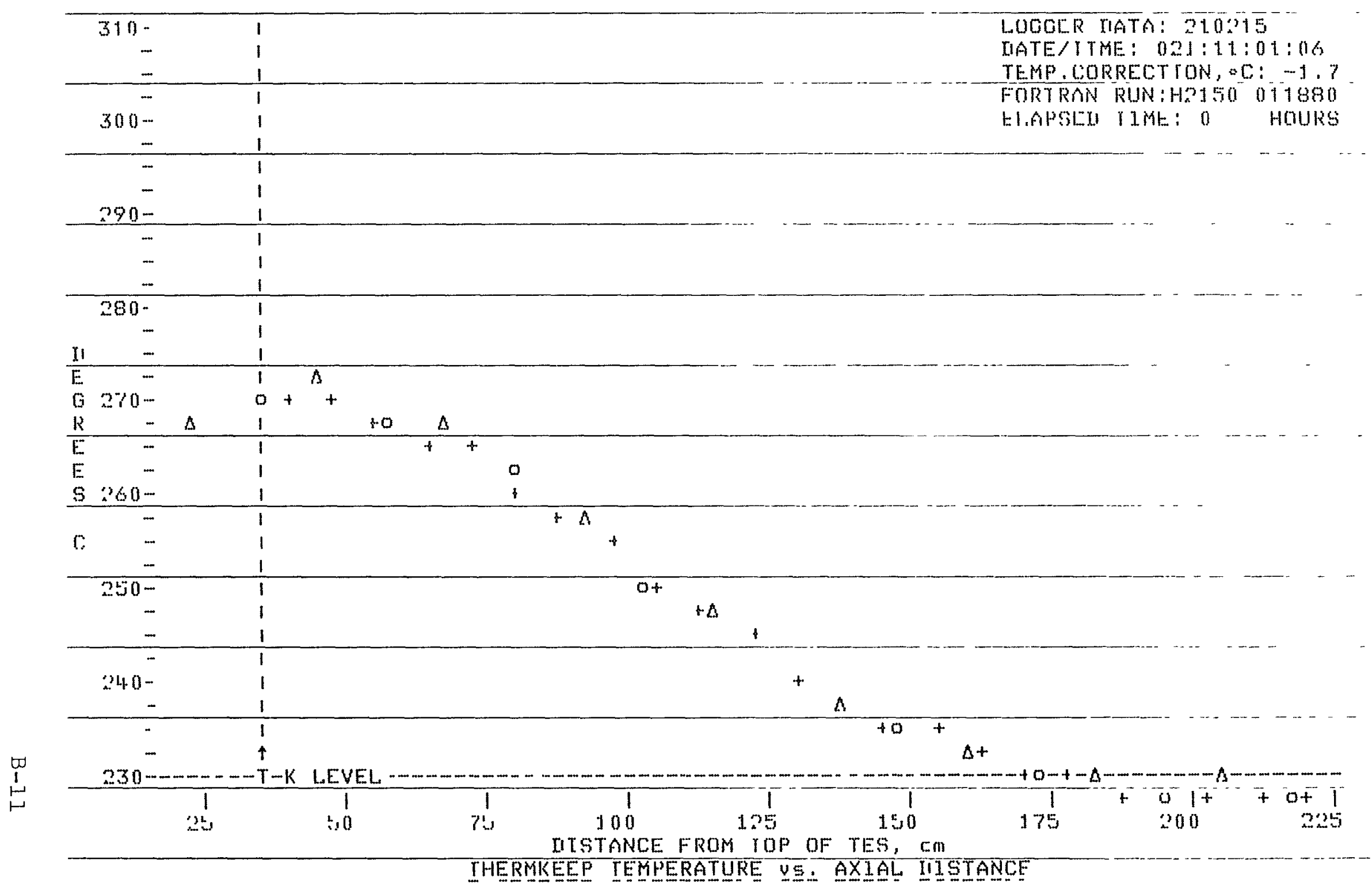

Figure B-9. Test No. 5 


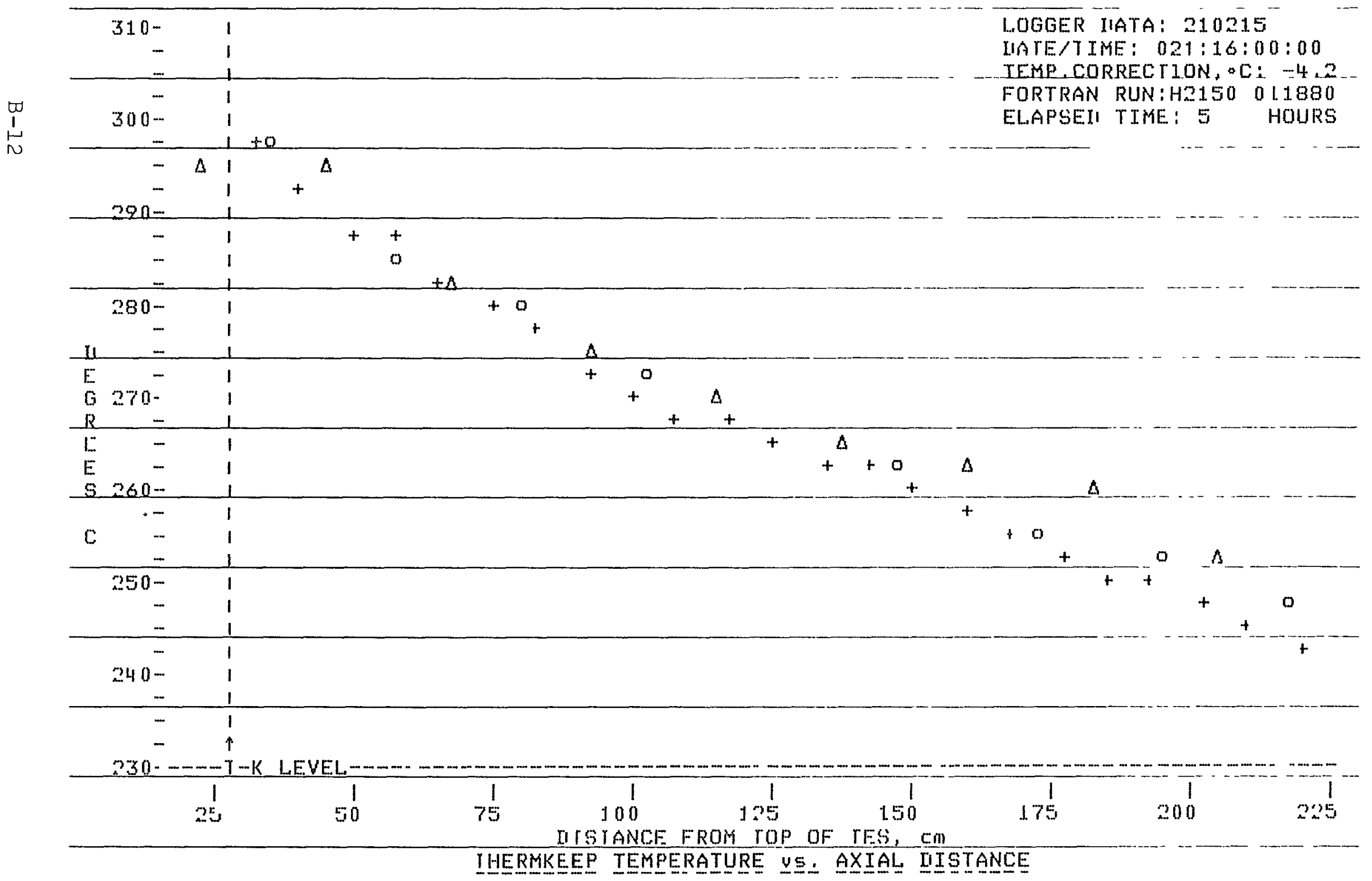

Figure B-10. Test No. 5. 


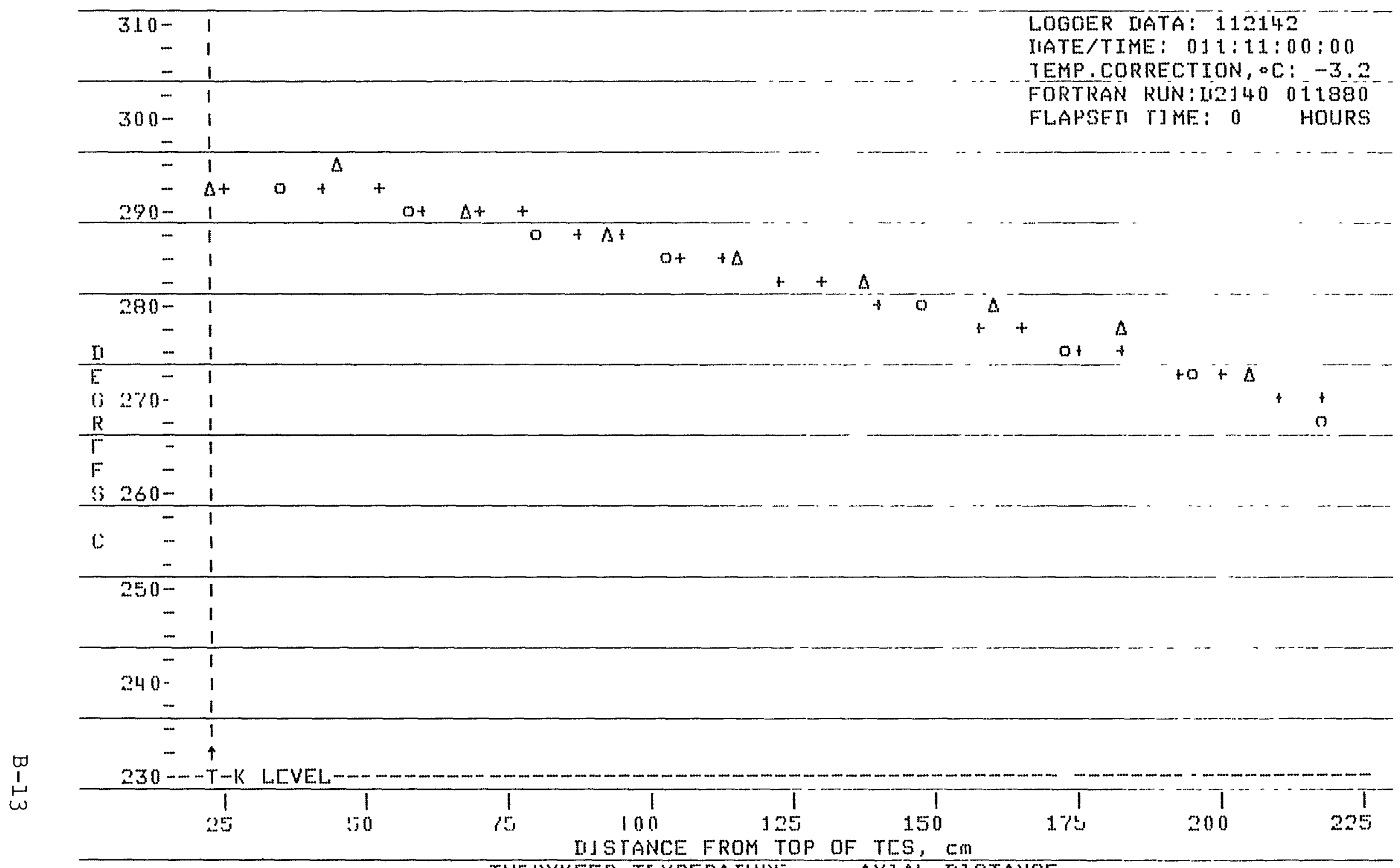

THERYKEEP IEMPERAIURI: YS. AXIAL IIISTANCE

Figure B-Il. Test No.6. 


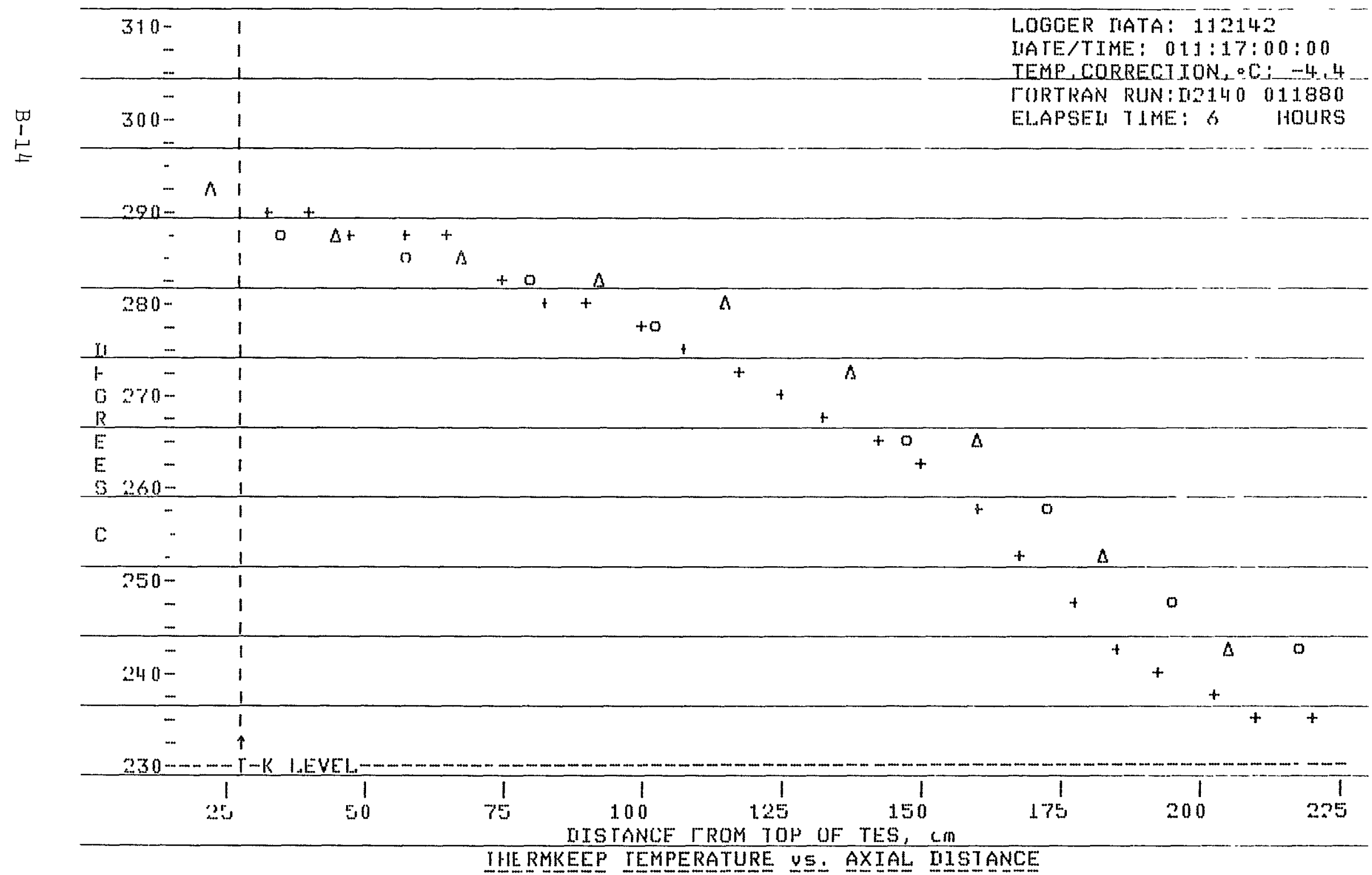

Figure B-12. Test No, 6 . 


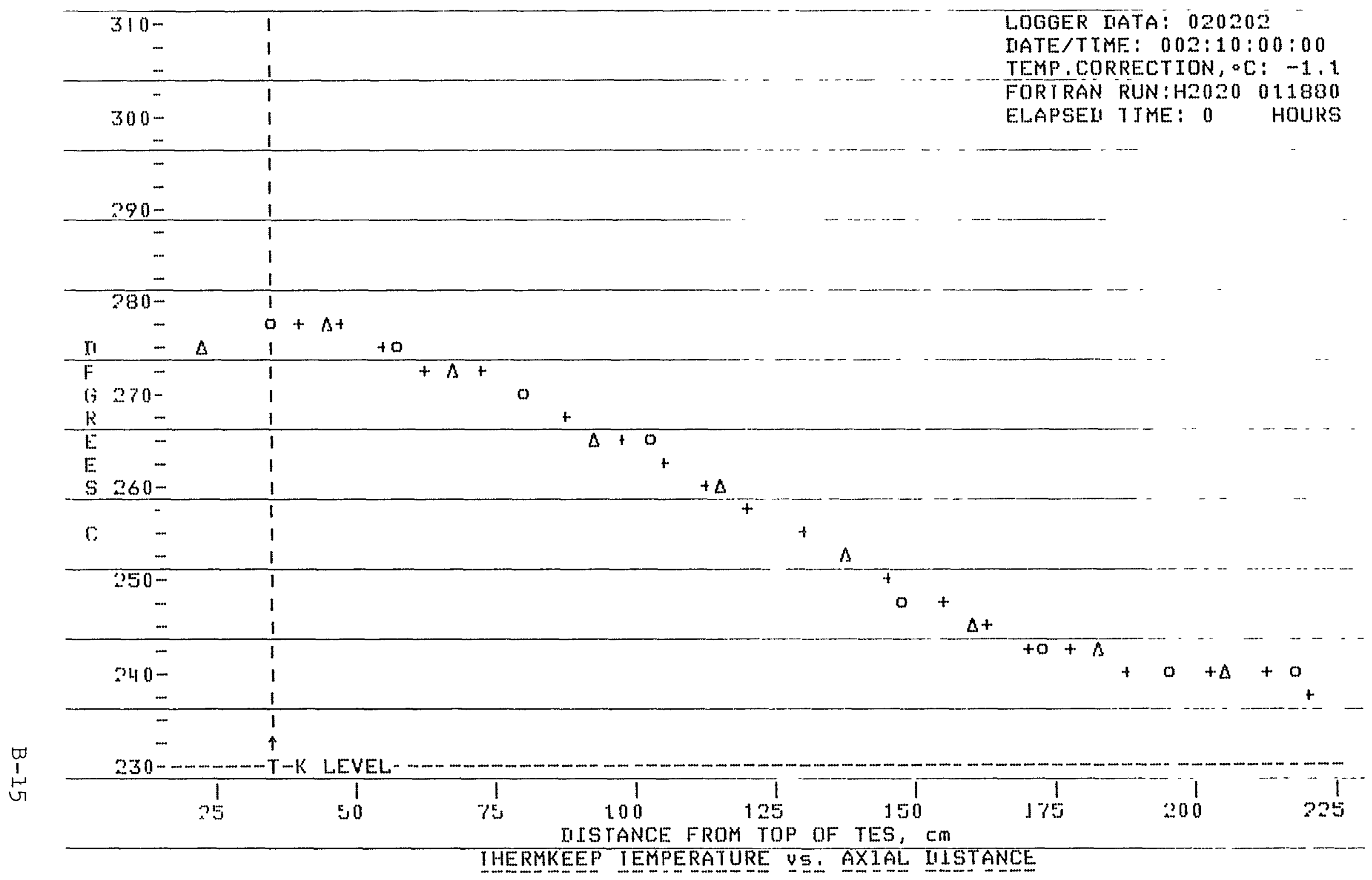

Figure B.13. Test No. 7 . 


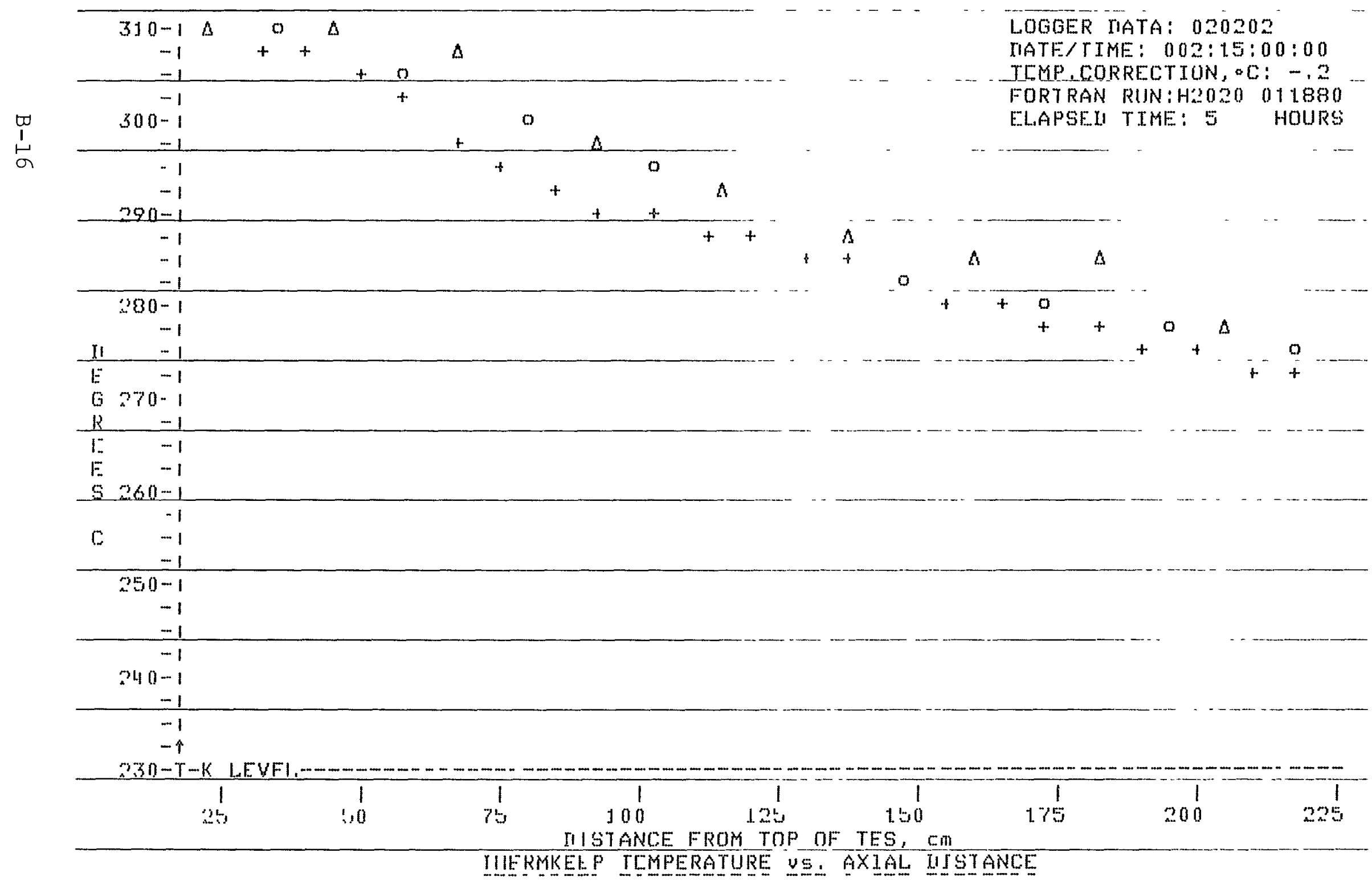

Figure B-14. Test No. 7 . 


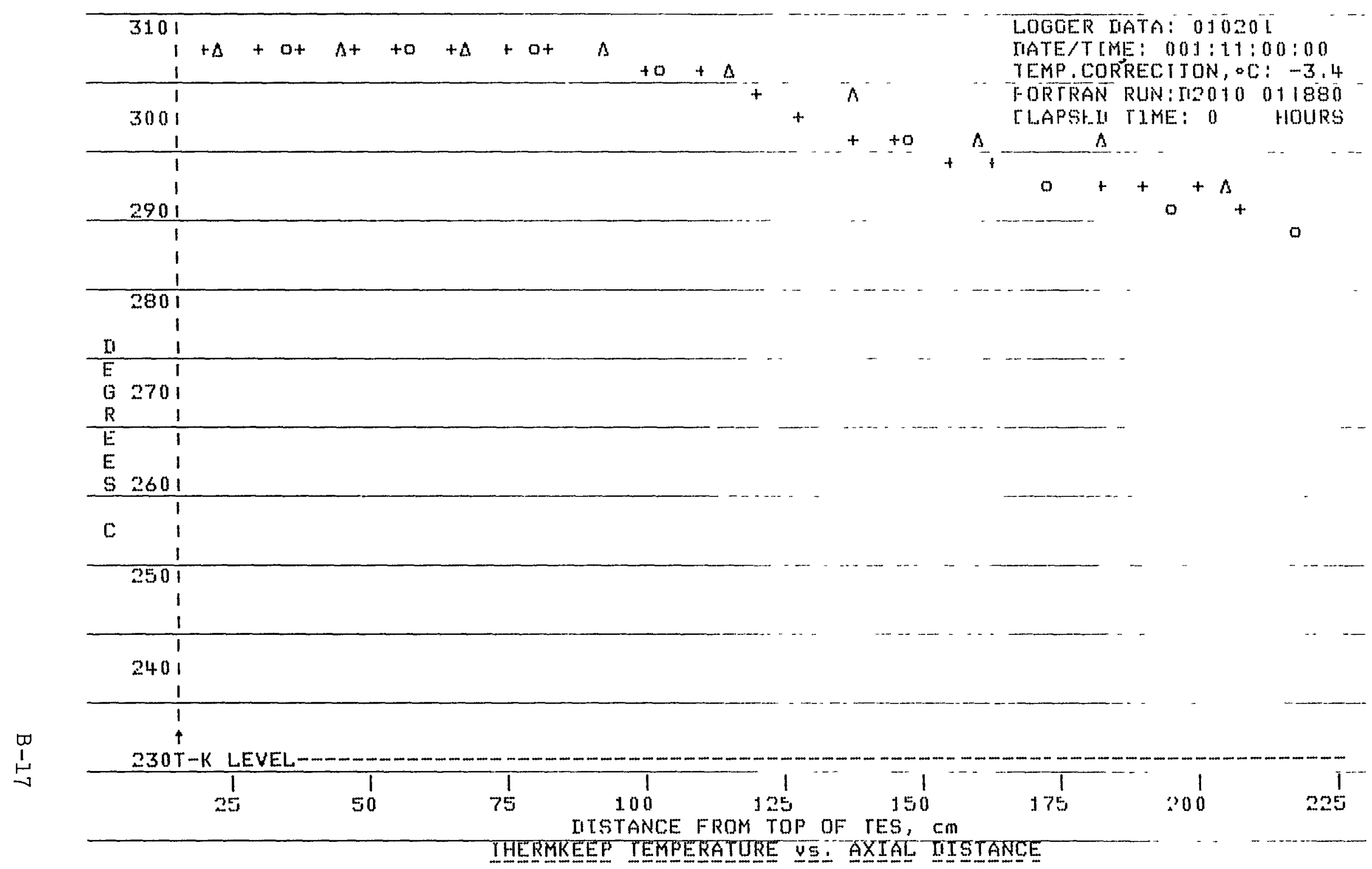

Figure B-15. Test No. 8 . 


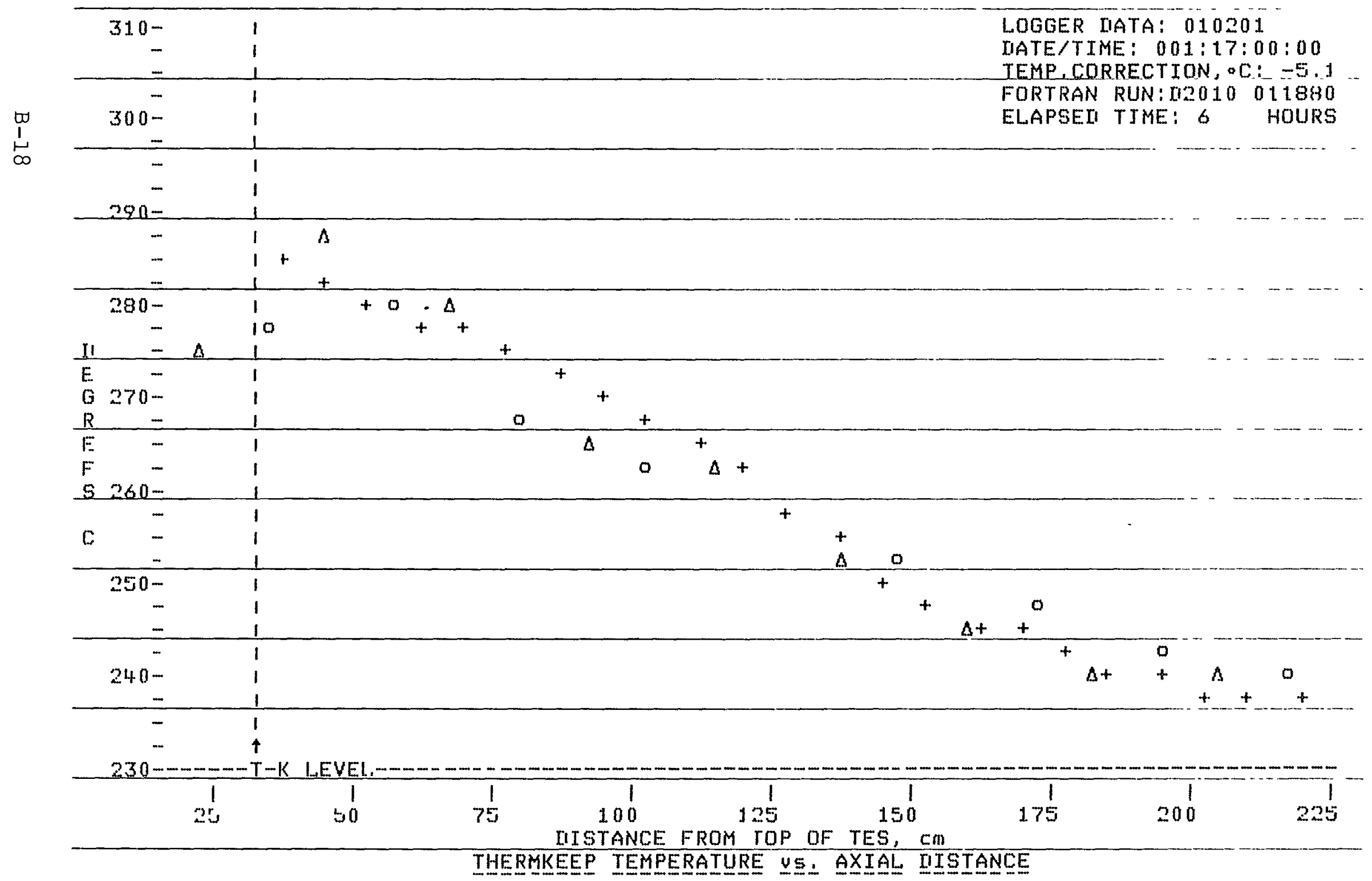

Figure B-16. Test No. 8 . 


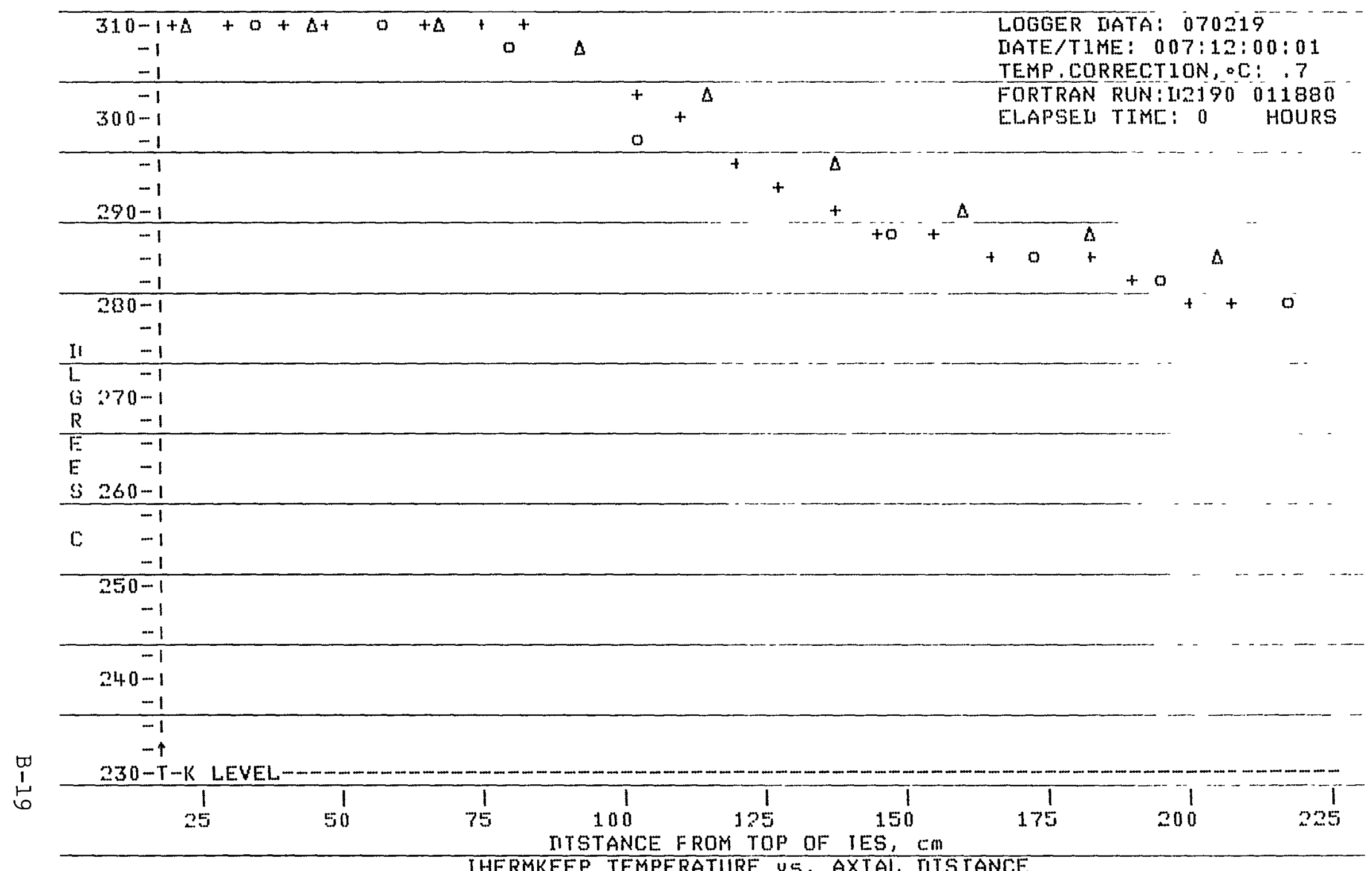

IHERMKEEP IEMPERATURE VS- AXIAL DISTANCE

Figure B-17. Test No. 9 


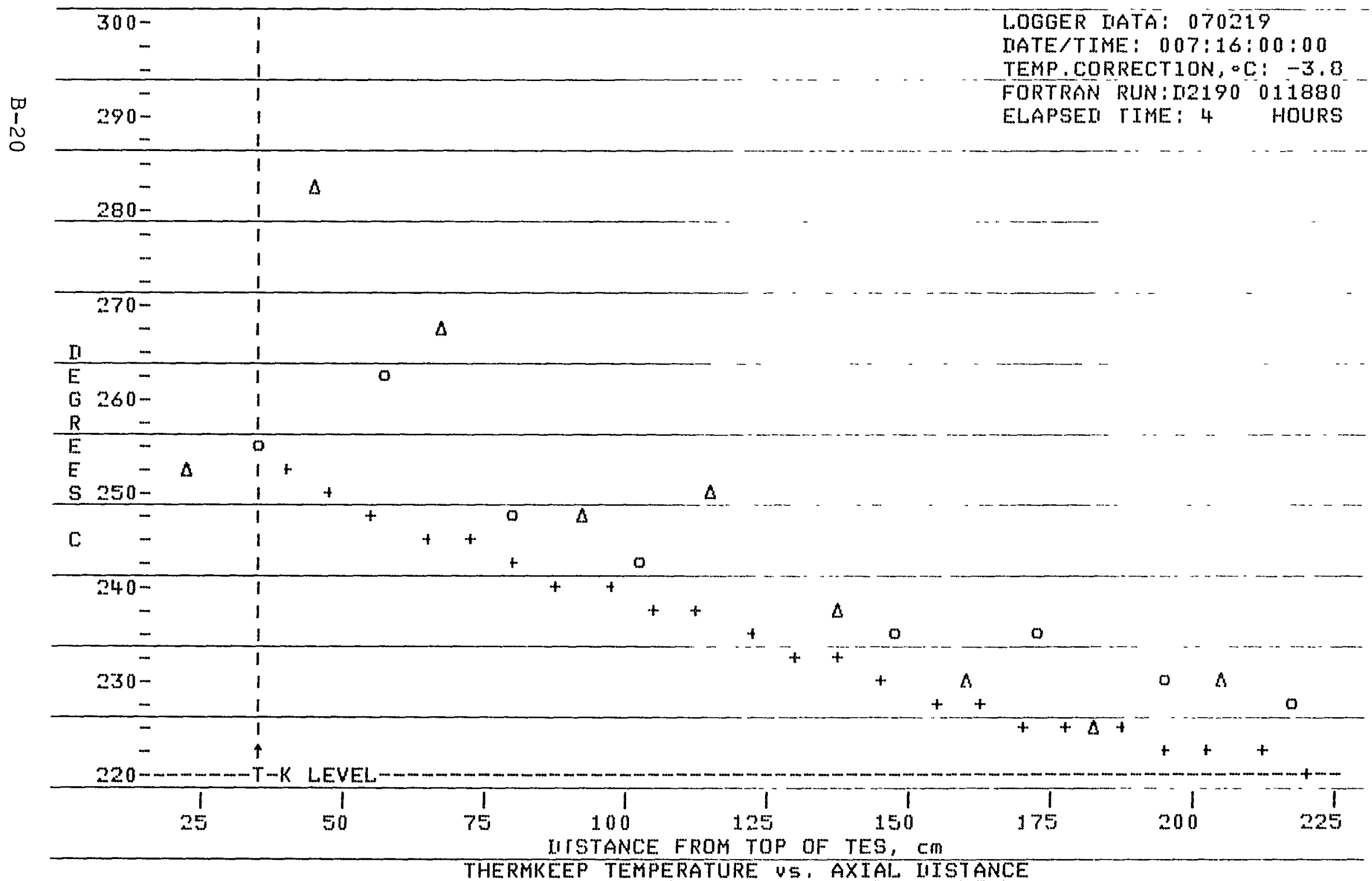

Figure B-18. Test No, 2. 
A P P E N D I X C

DATA CORRELATIONS

C-1 


\section{SYMBOLS}

Heat transfer fluid ( $\mathrm{HT}-43$ and $\mathrm{T}-66$ ) temperatures:

- = Experimental data

- = Computer model predictions

Thermkeep temperatures:

o = Shallow well temperature vs. location in tank

$\Delta=$ Deep well temperature vs. locations in tank

$\square=$ Tank surface temperature vs. location in tank

$+=$ Computer model prediction 


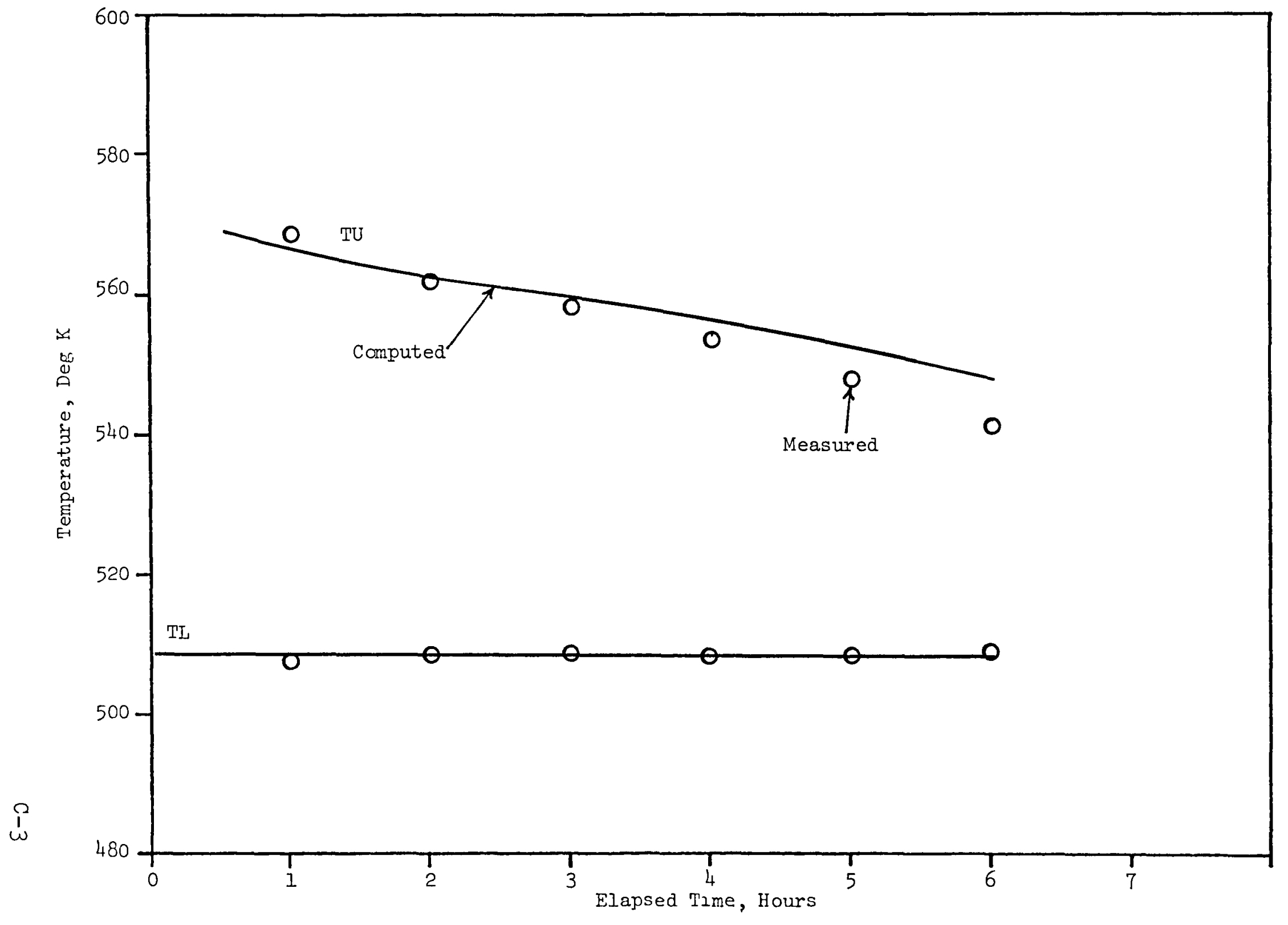

Figure C-1. HT-43 outlet temperature, discharge test 201 (3.3 gpm) 


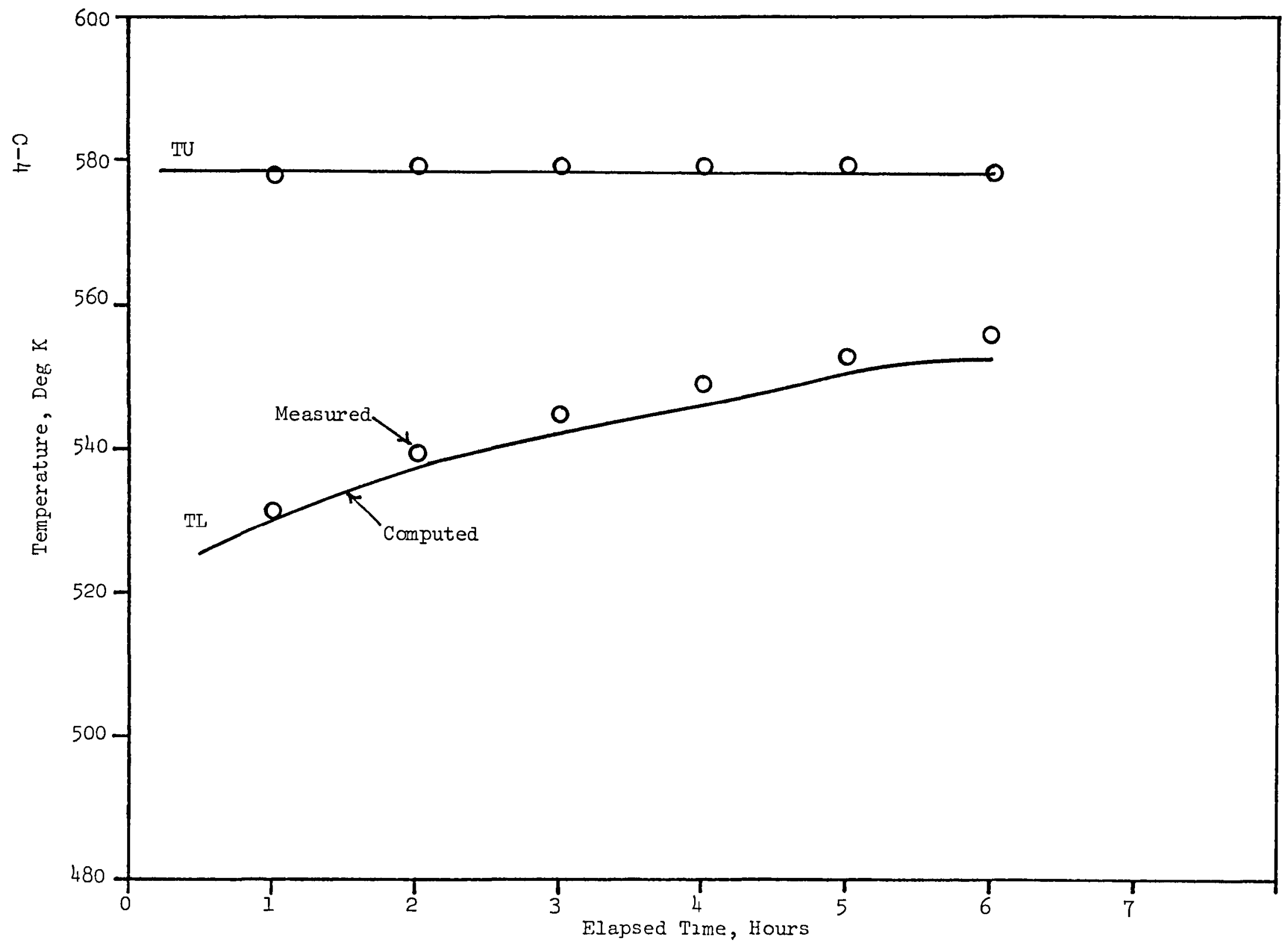

Figure C-2. HT-43 outlet temperature, charge test 202 ( $3.3 \mathrm{gpm}$ ) 


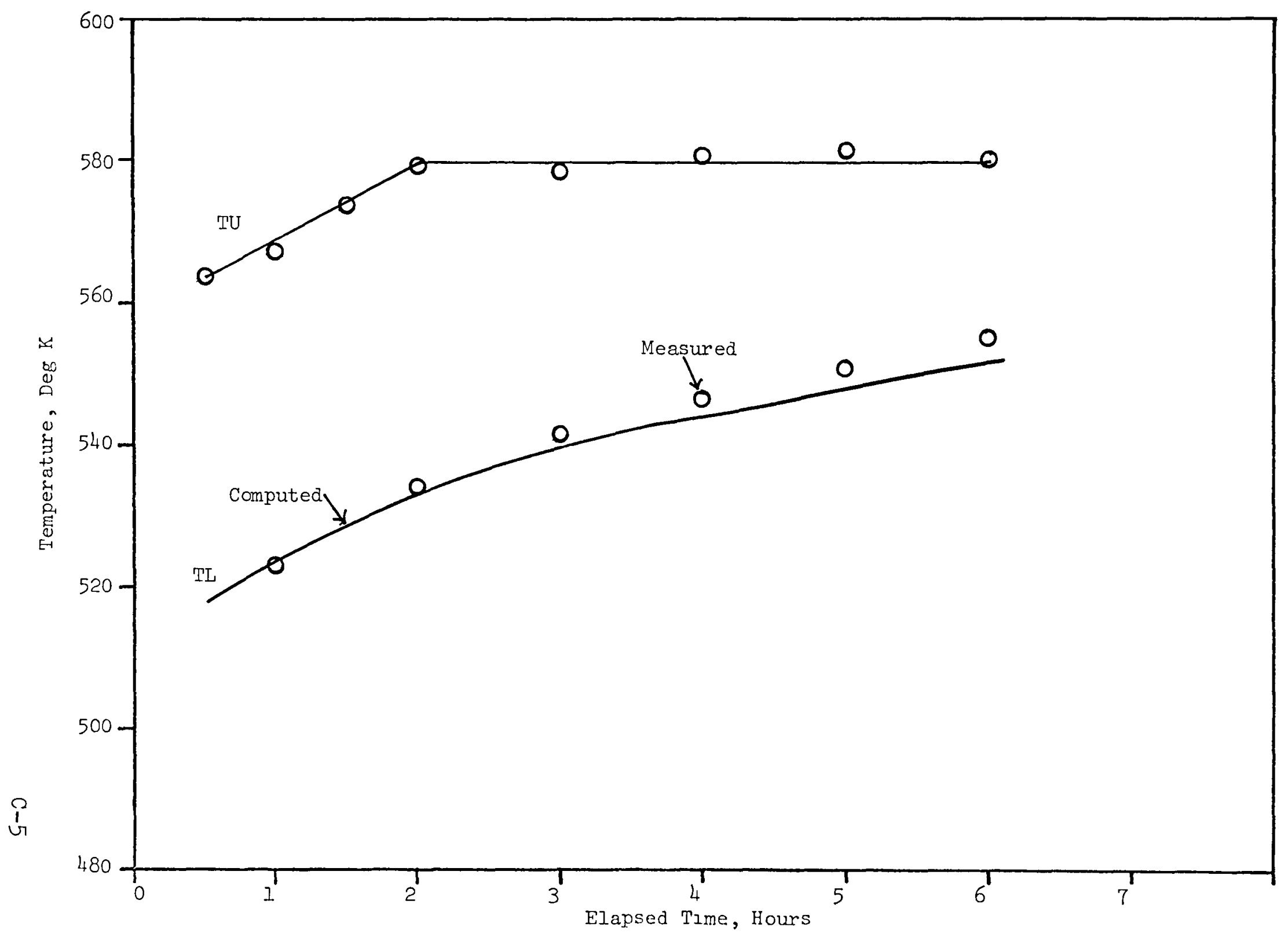

Figure C-3. HT-43 outlet temperature, charge test 204 ( $3.7 \mathrm{gpm}$ ) 


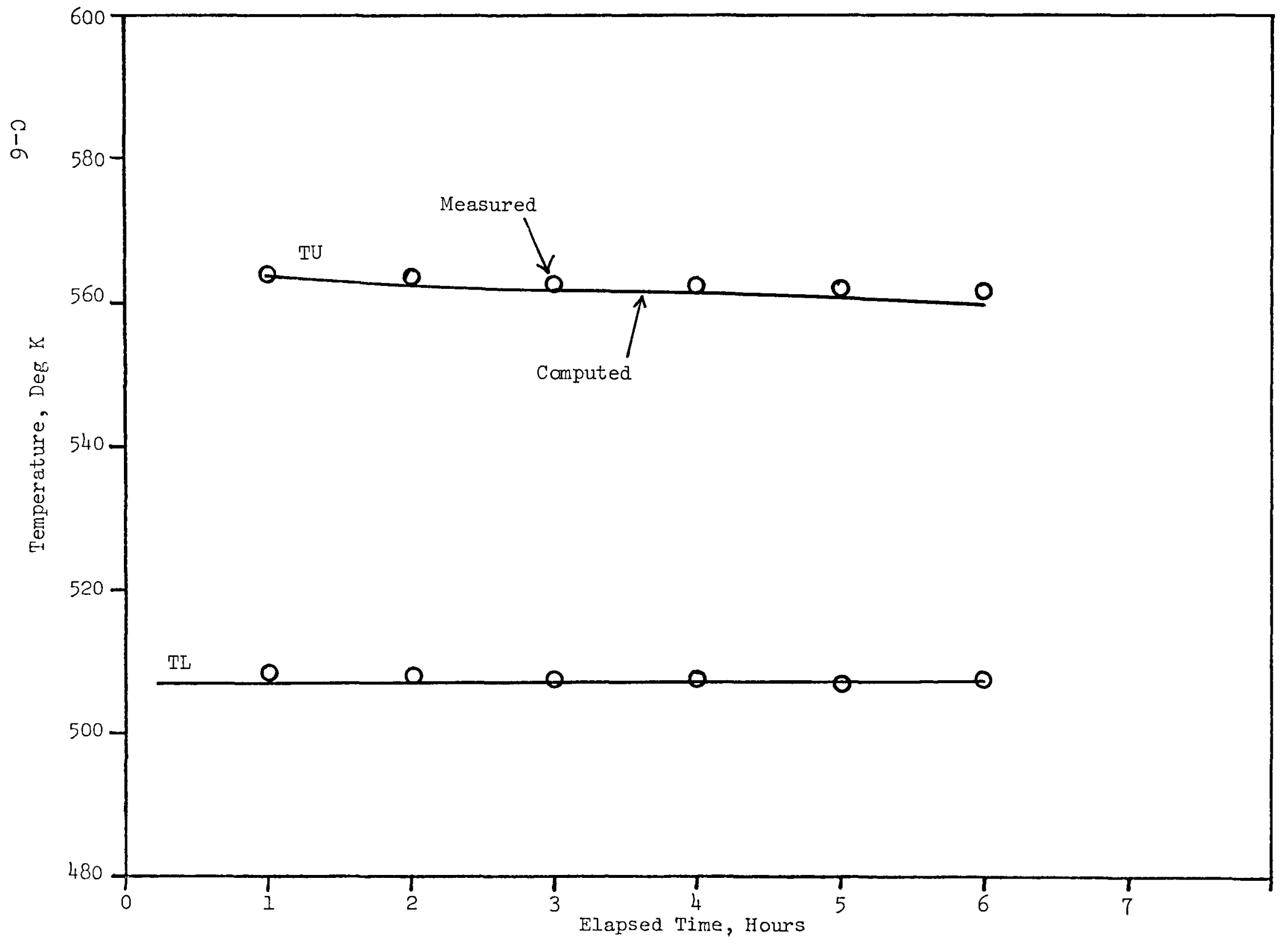

Fıgure C-4. HT-43 outlet temperature, discharge test 214-2 (1.1 gpm) 


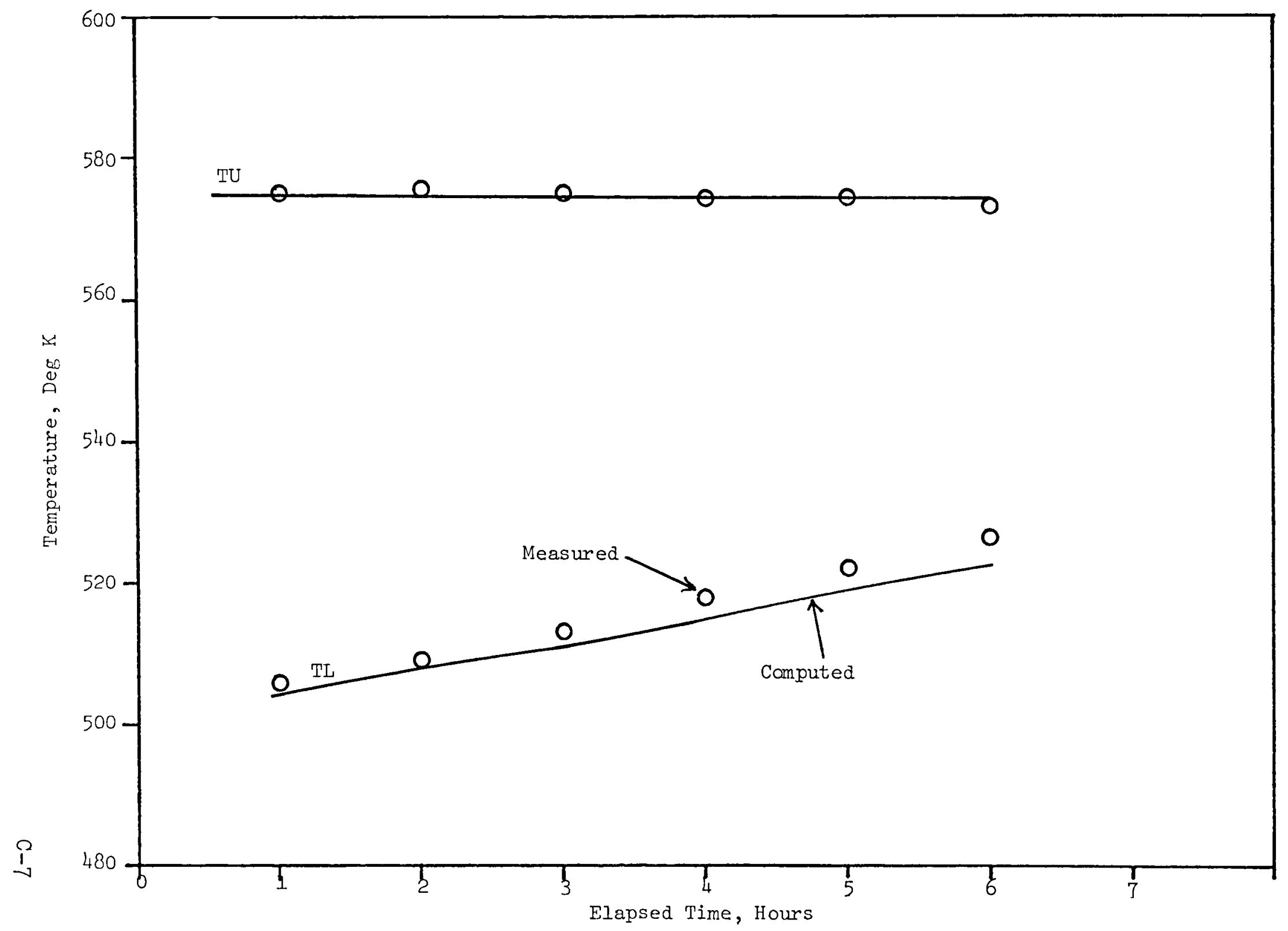

Figure C-5. HT-43 outlet temperature, charge test 215-2 ( $1.1 \mathrm{gpm}$ ) 


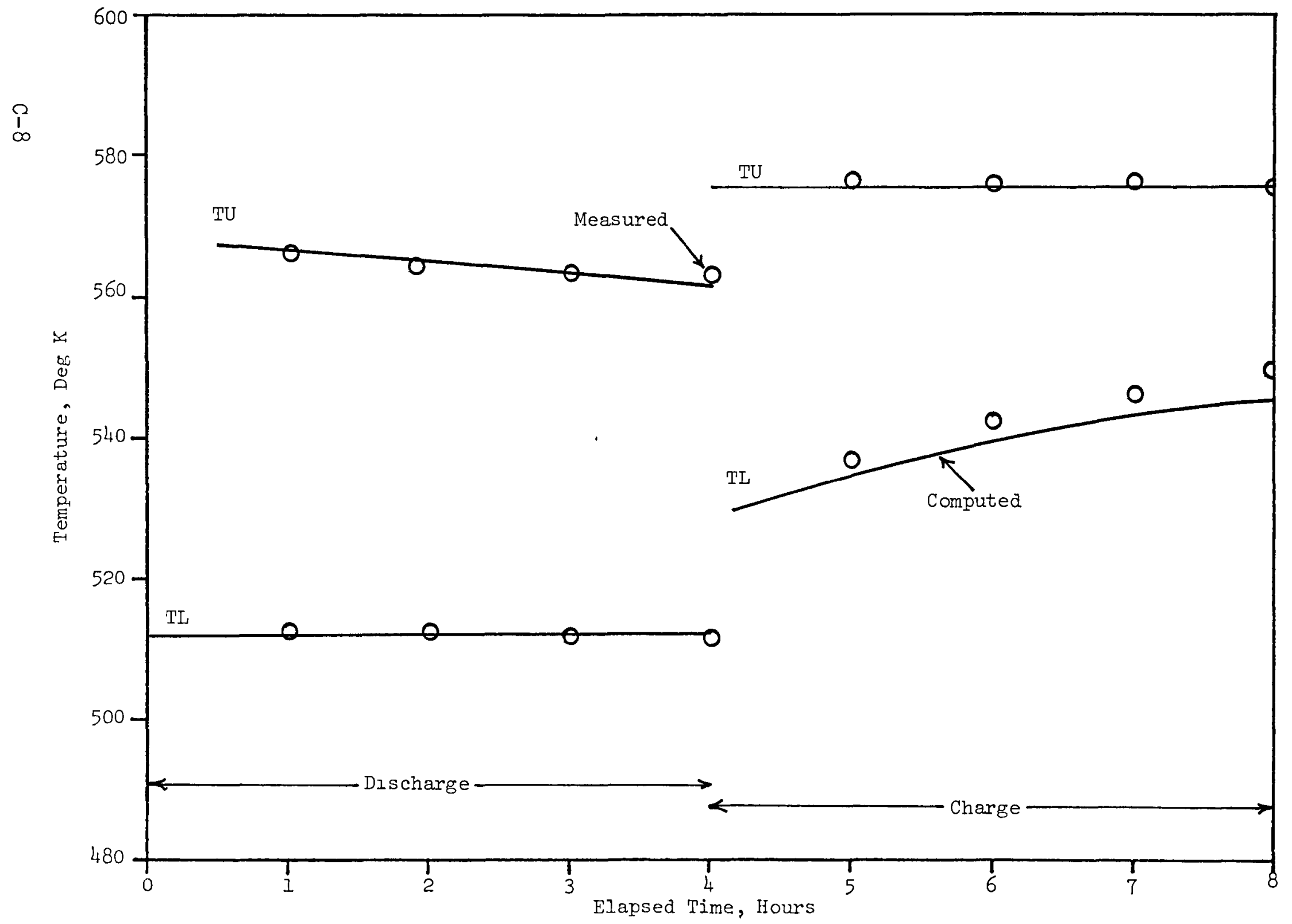

Figure C-6. HT-43 outlet temperature, cyclic test 218 ( $1.9 \mathrm{gpm}$ ), first cycle 


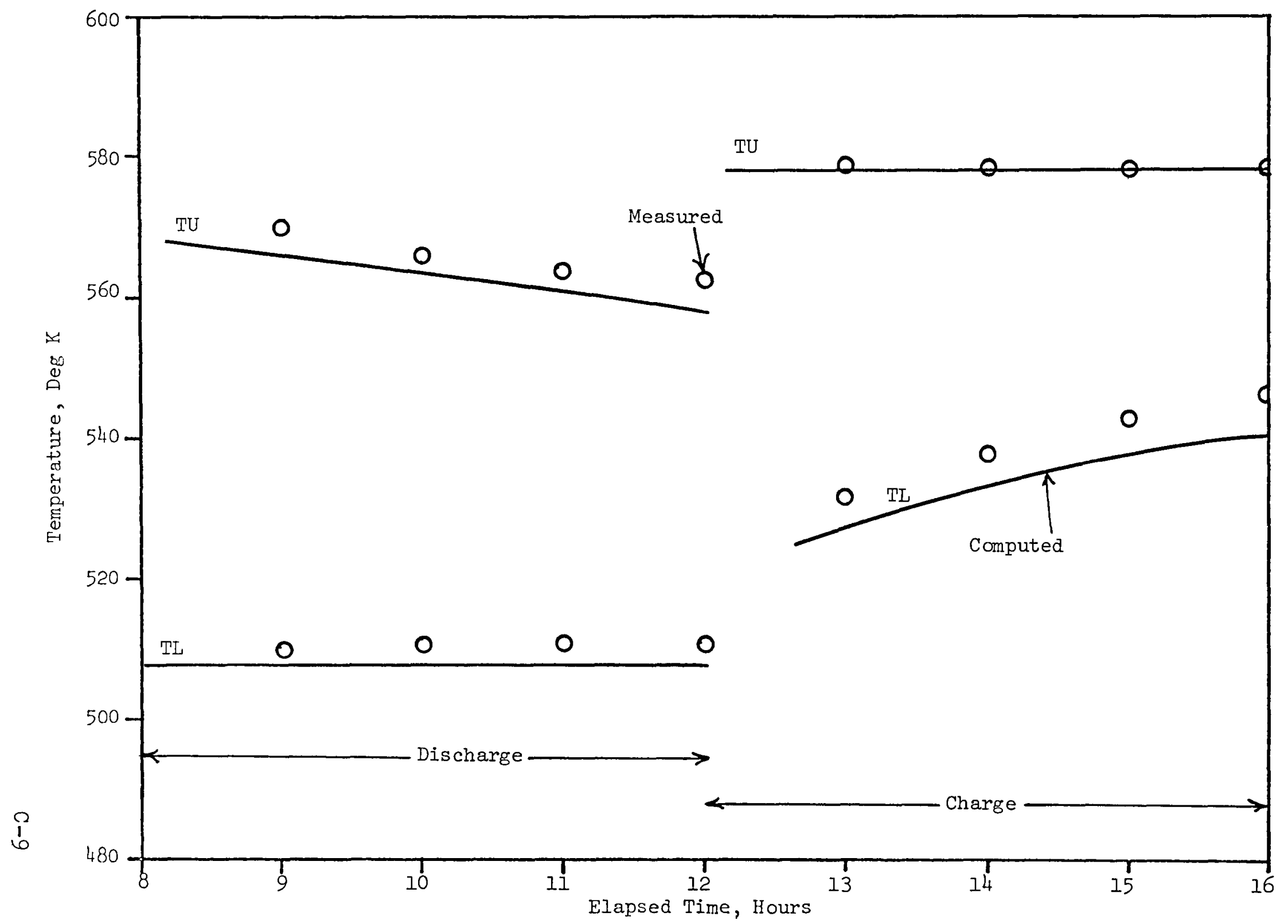

Figure C-7. HT-43 outlet temperature, cyclic test 218 ( $1.9 \mathrm{gpm})$, second cycle. 


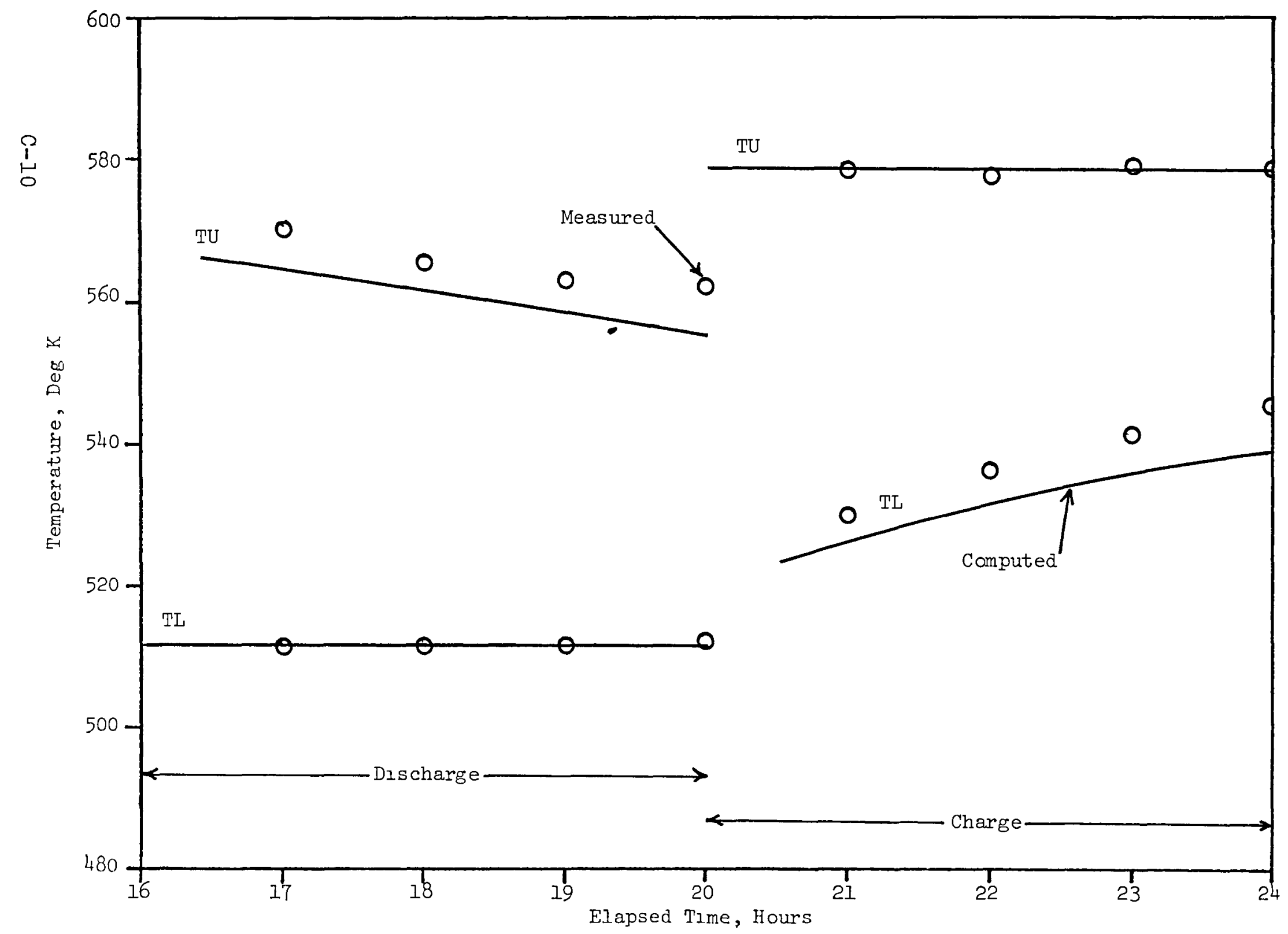

Figure C-8. HT-43 outlet temperature, cyclic test 218 (1.9 g pm), third cycle. 


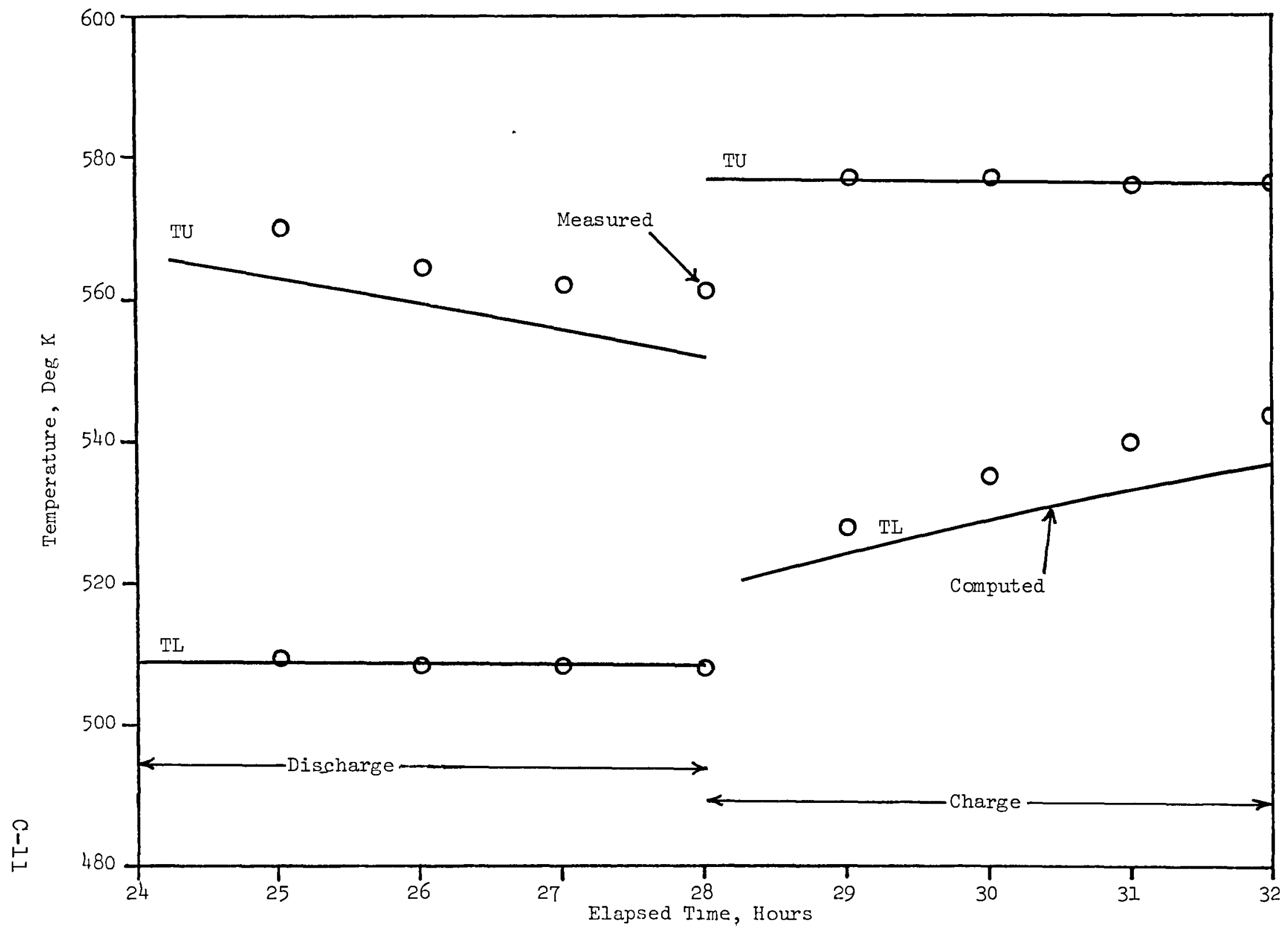

Figure C-9. HT-43 outlet temperature, cyclic test 218 ( $1.9 \mathrm{gpm}$ ), fourth cycle. 


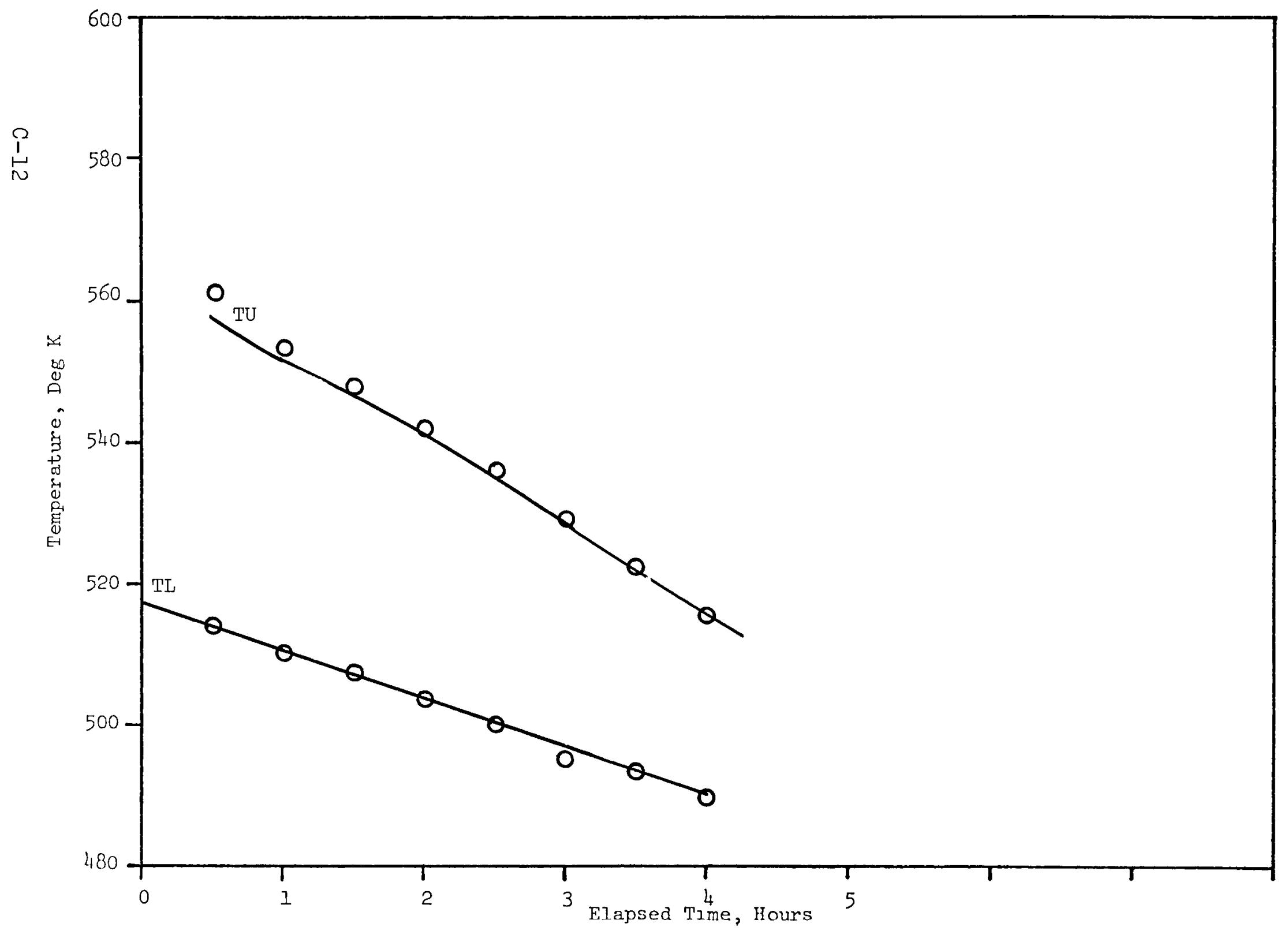

Figure C-10. HT-43 outlet temperature, discharge test $212(7.7 \mathrm{gpm})$. 


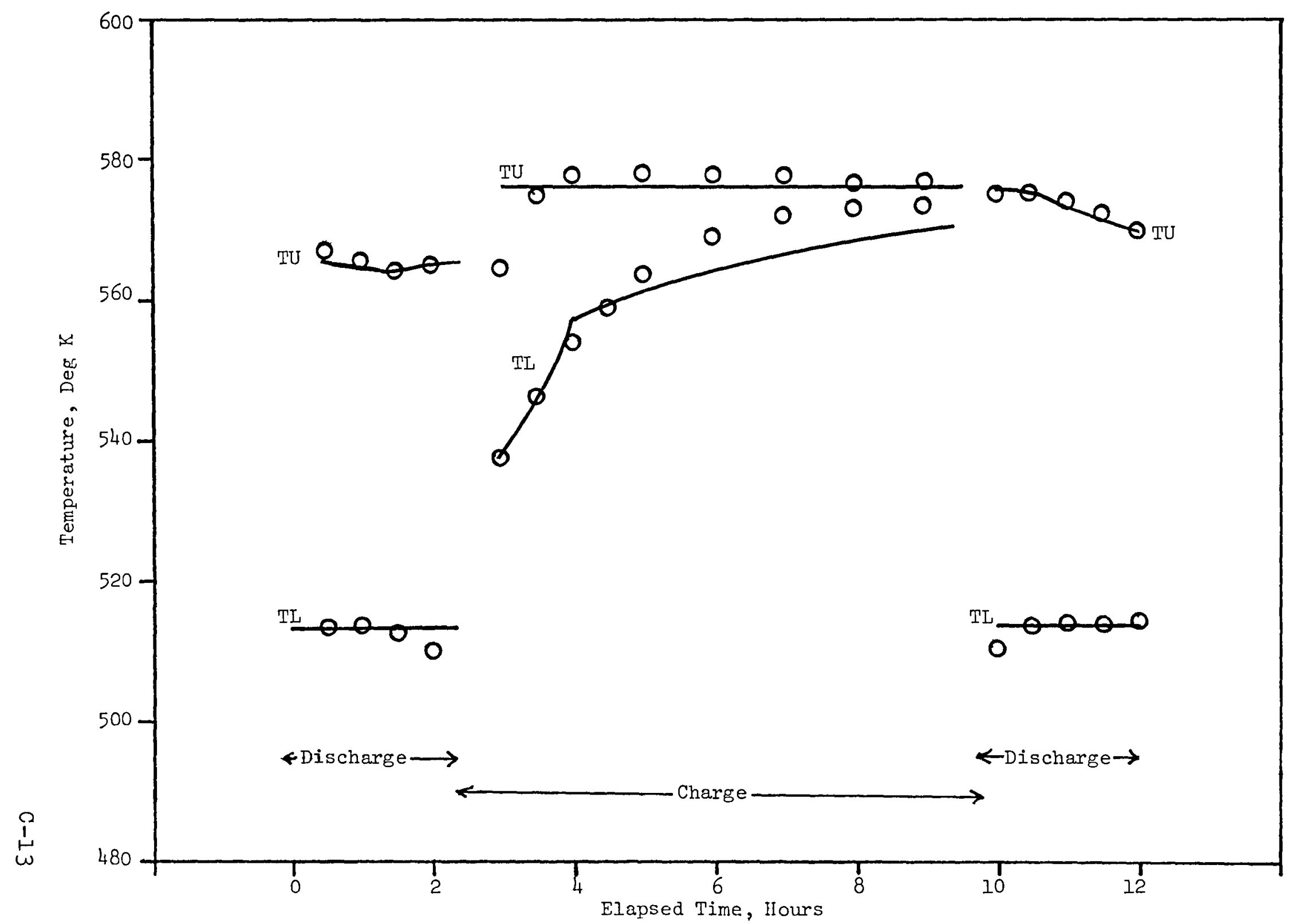

Figure C-11. HT-43 outlet temperature, solar cycle test 223, first cycle. 


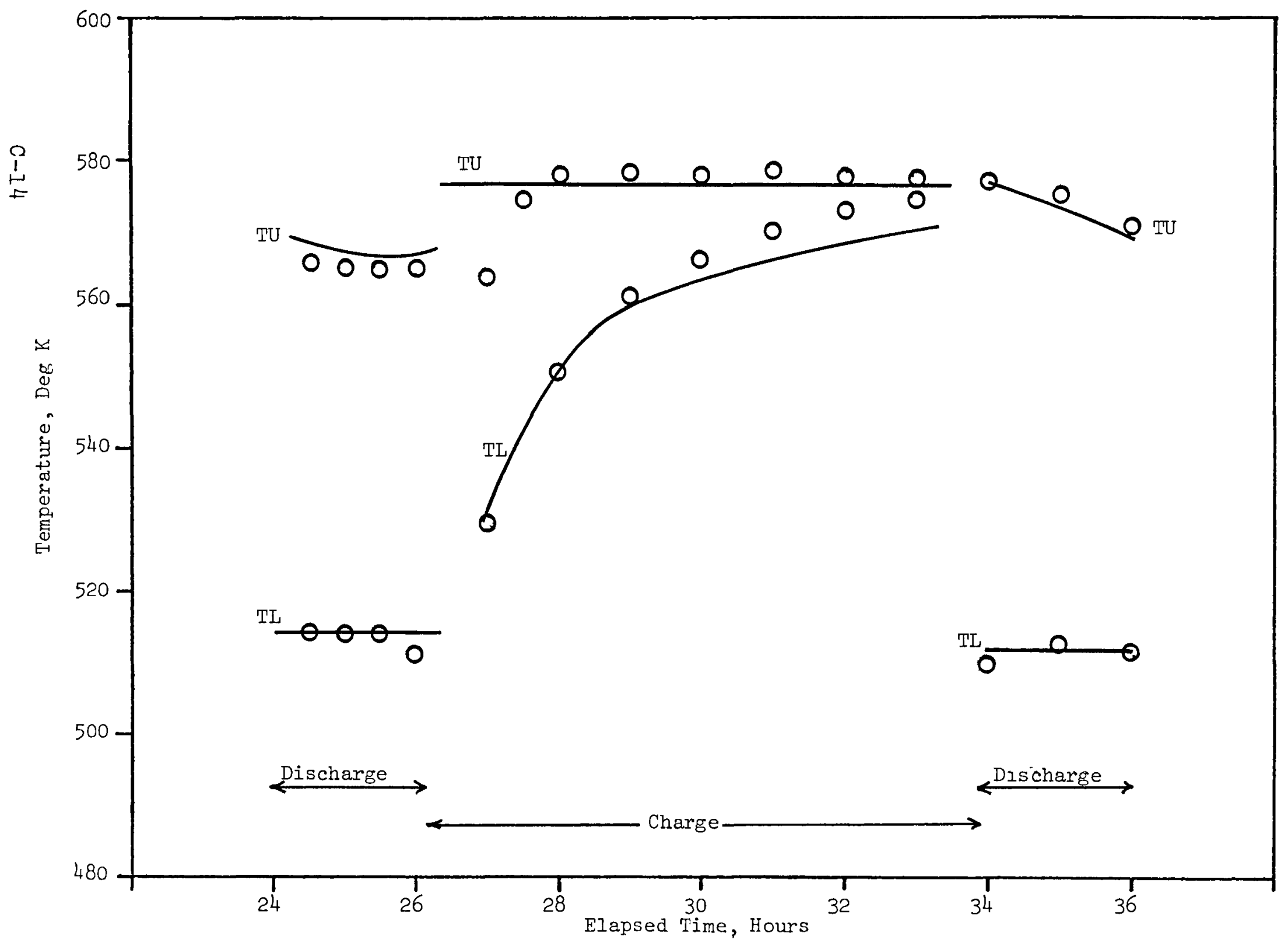

Figure C-12. HT-43 outlet temperature, solar cycle test 223 , second cycle. 


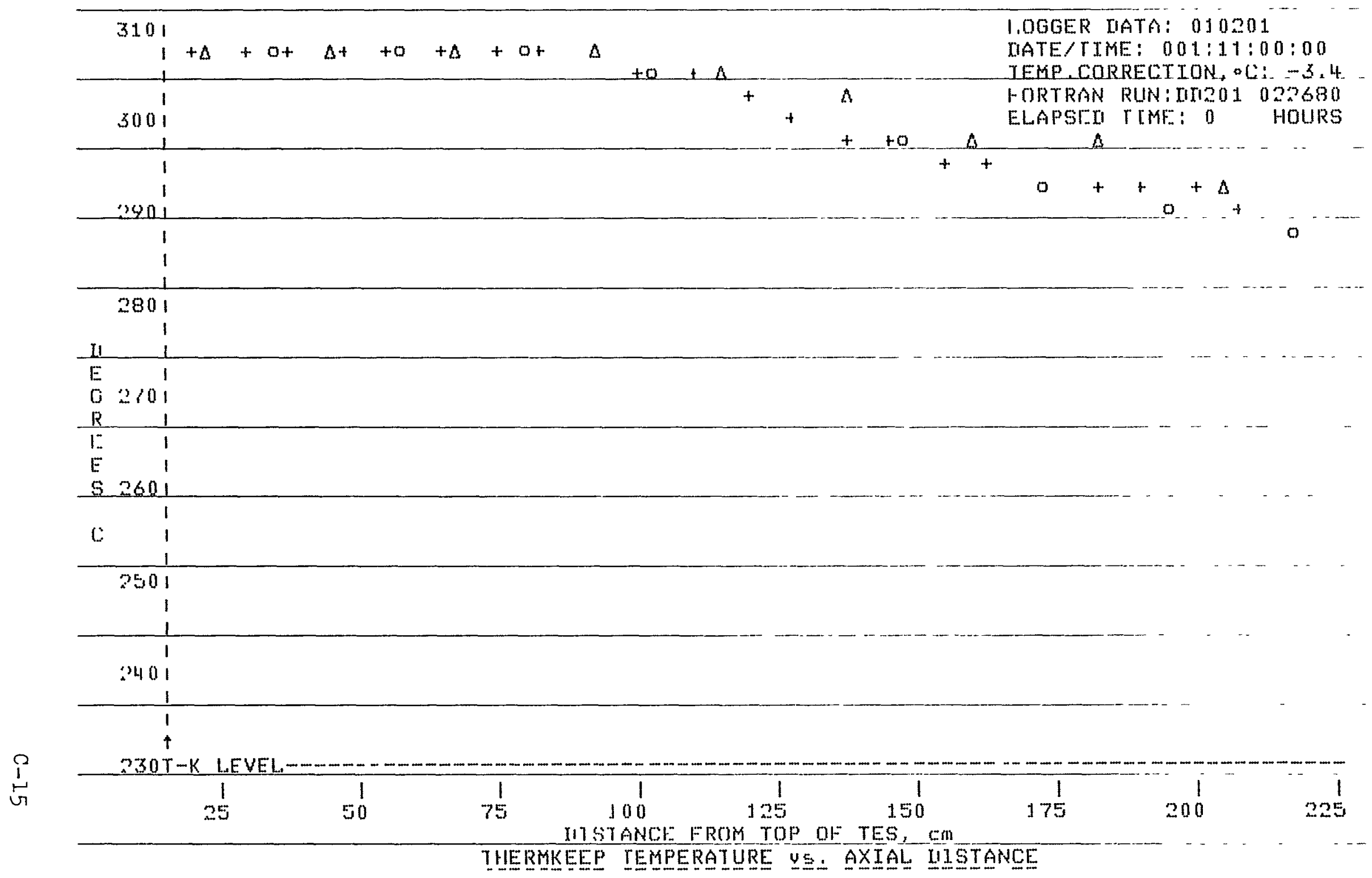

Figure C-13. Thermkeep temperature profile, discharge test 201 ( $3.3 \mathrm{gpm}$ ), starting profile. 


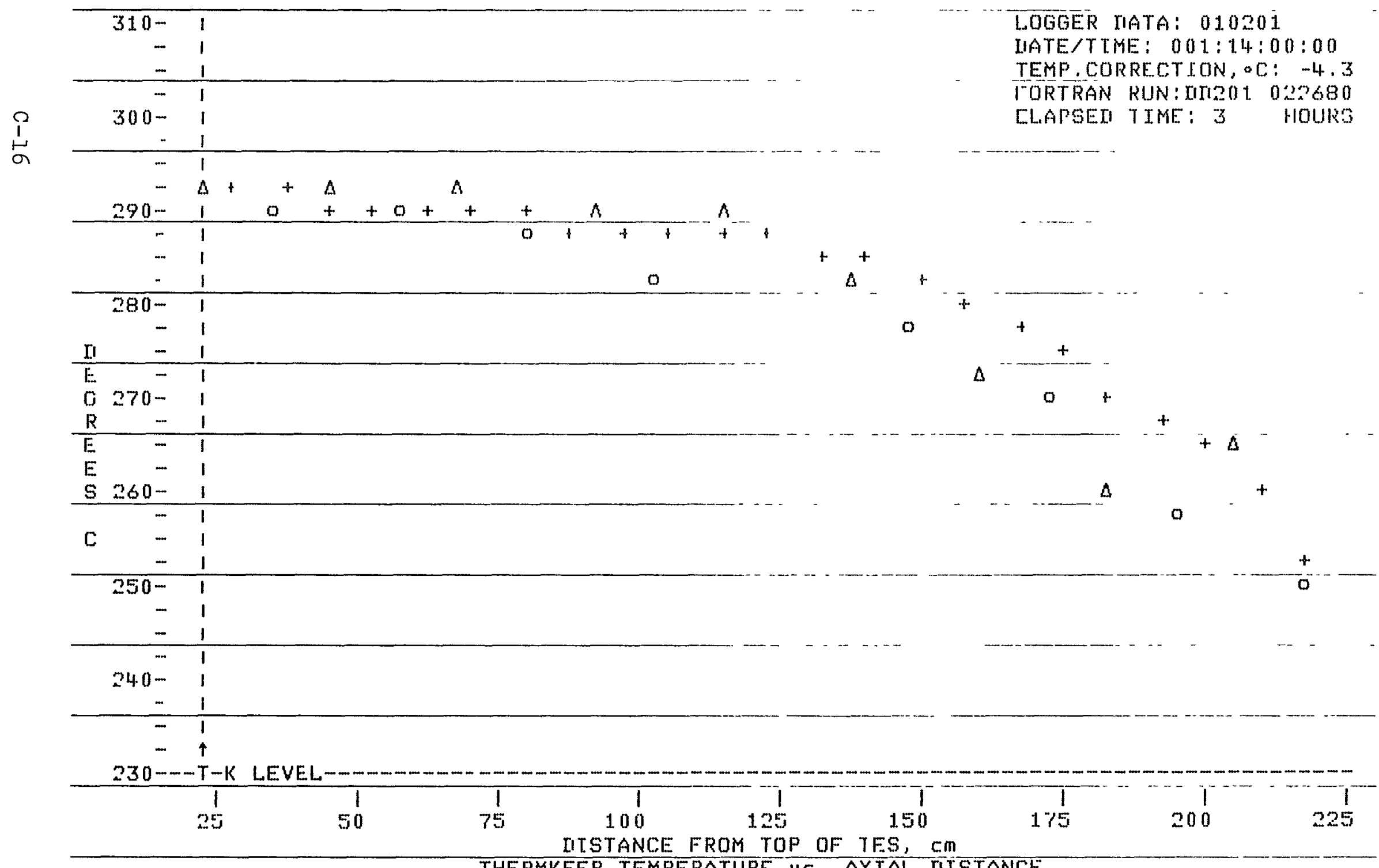

THERMKEEP IEMPERATURE VS- AXIAL IIISTANCE

Figure C-14, Thermkeep temperature profile, discharge test 201 (3.3 gpm), after 3 hours. 


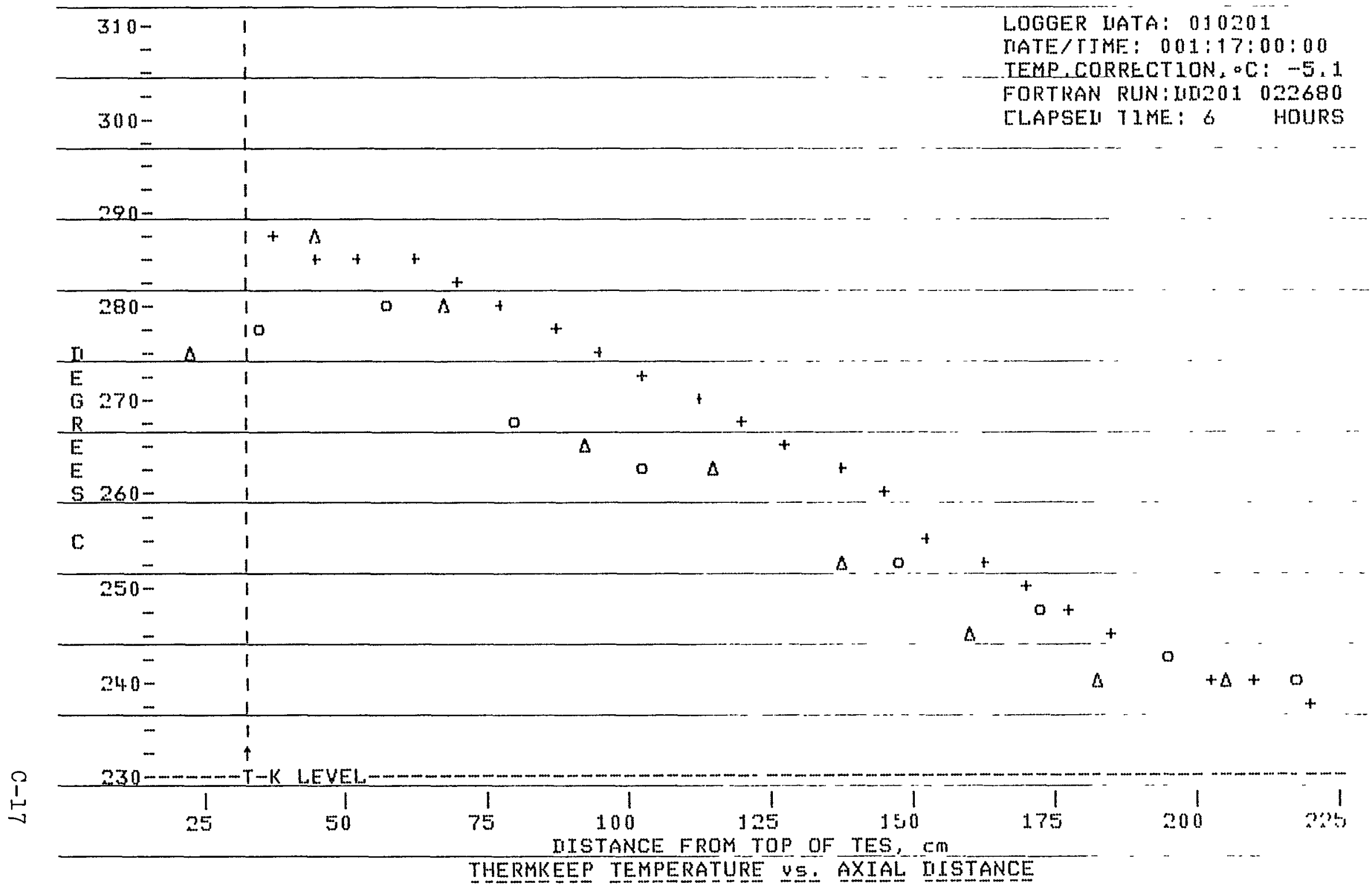

Figure C-15. Thermkeep temperature proflle, discharge test 201 ( $3.3 \mathrm{gpm}$ ), after 6 hours. 


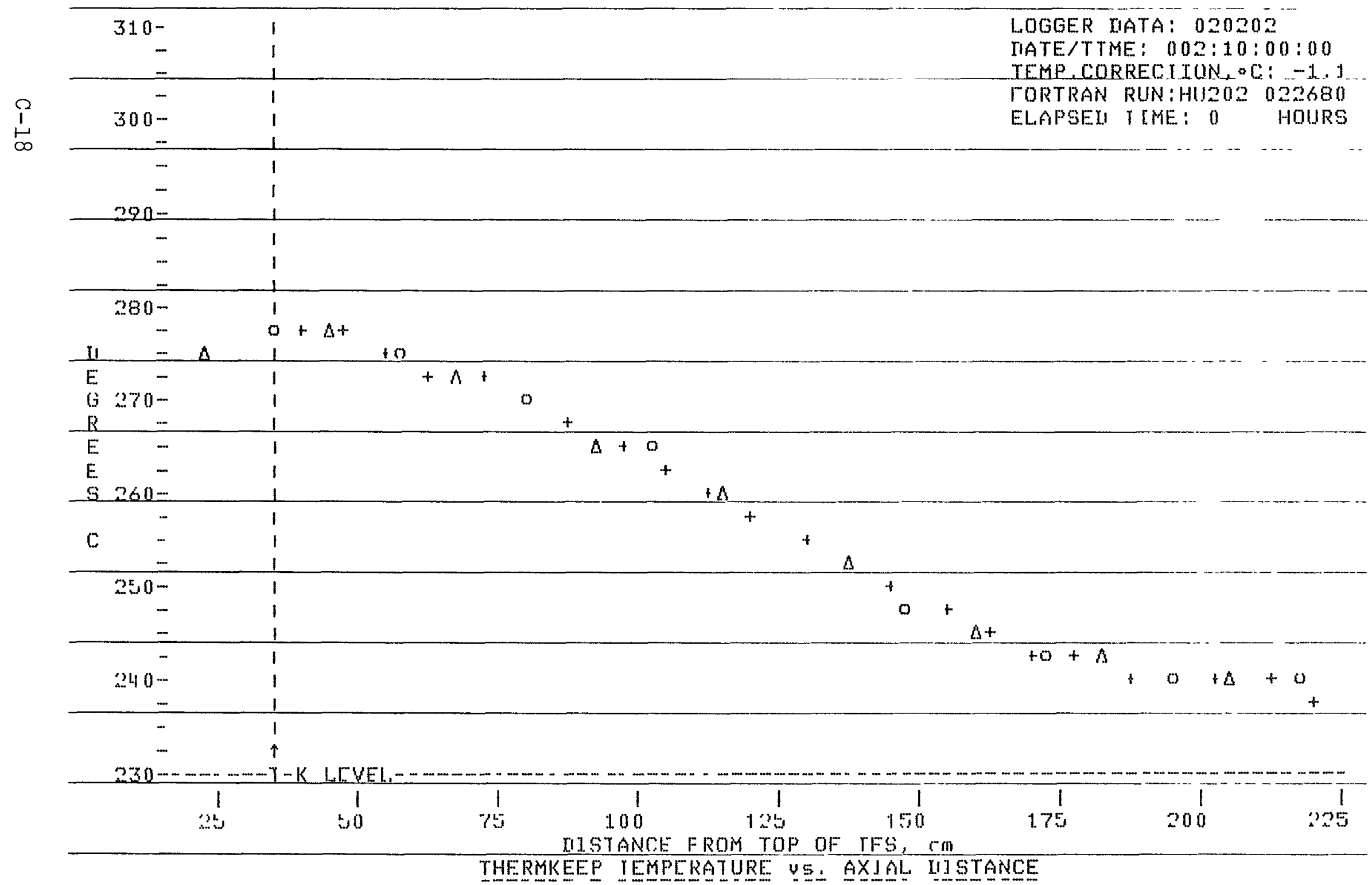

Figure C-16. Thermkeep temperature profile, charge test 202 (3,3 gpm), starting profile, 


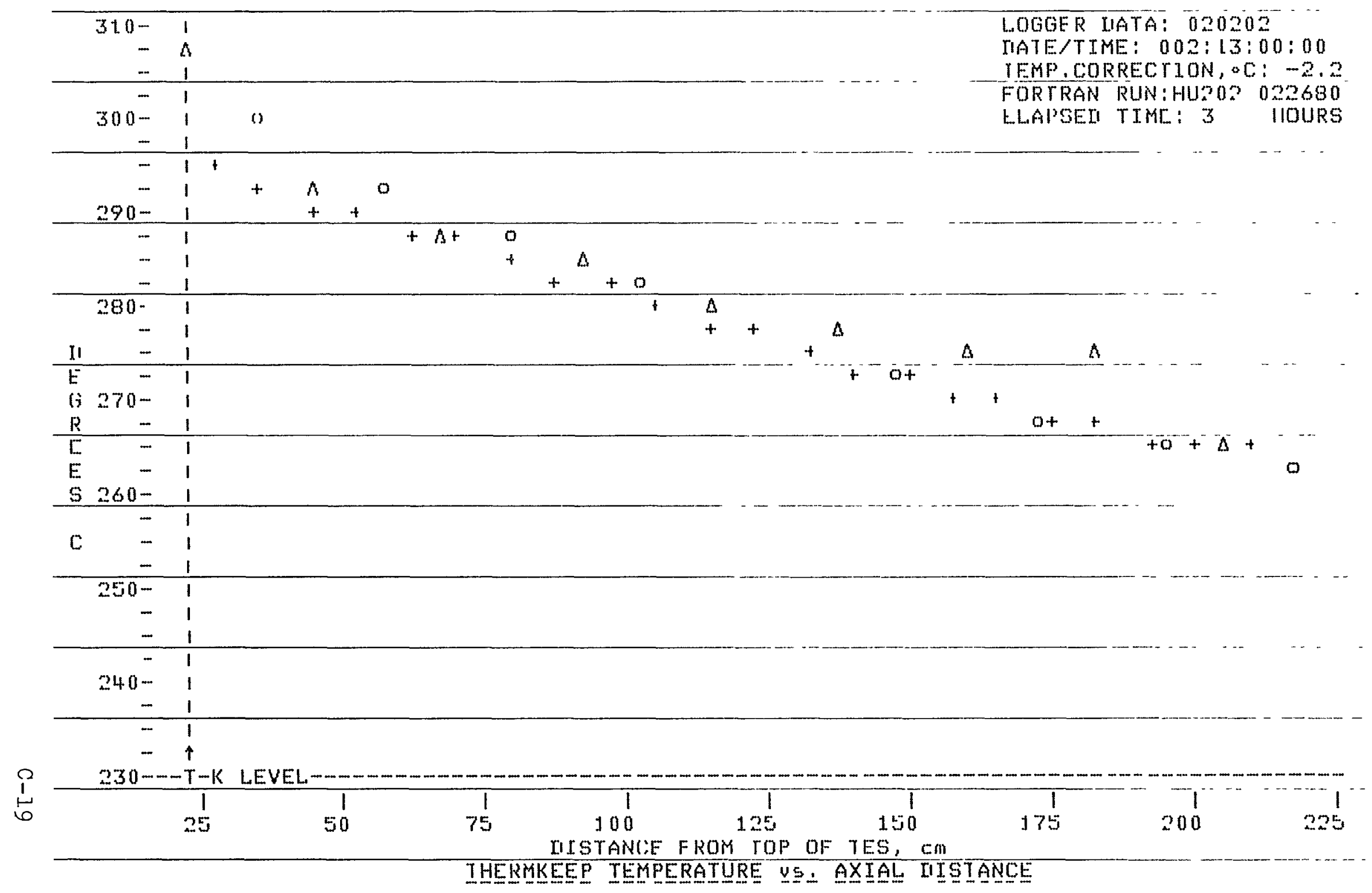

Figure C-17. Thermkeep temperature profile, charge test 202 ( $3.3 \mathrm{gpm}$ ), after 3 hours. 


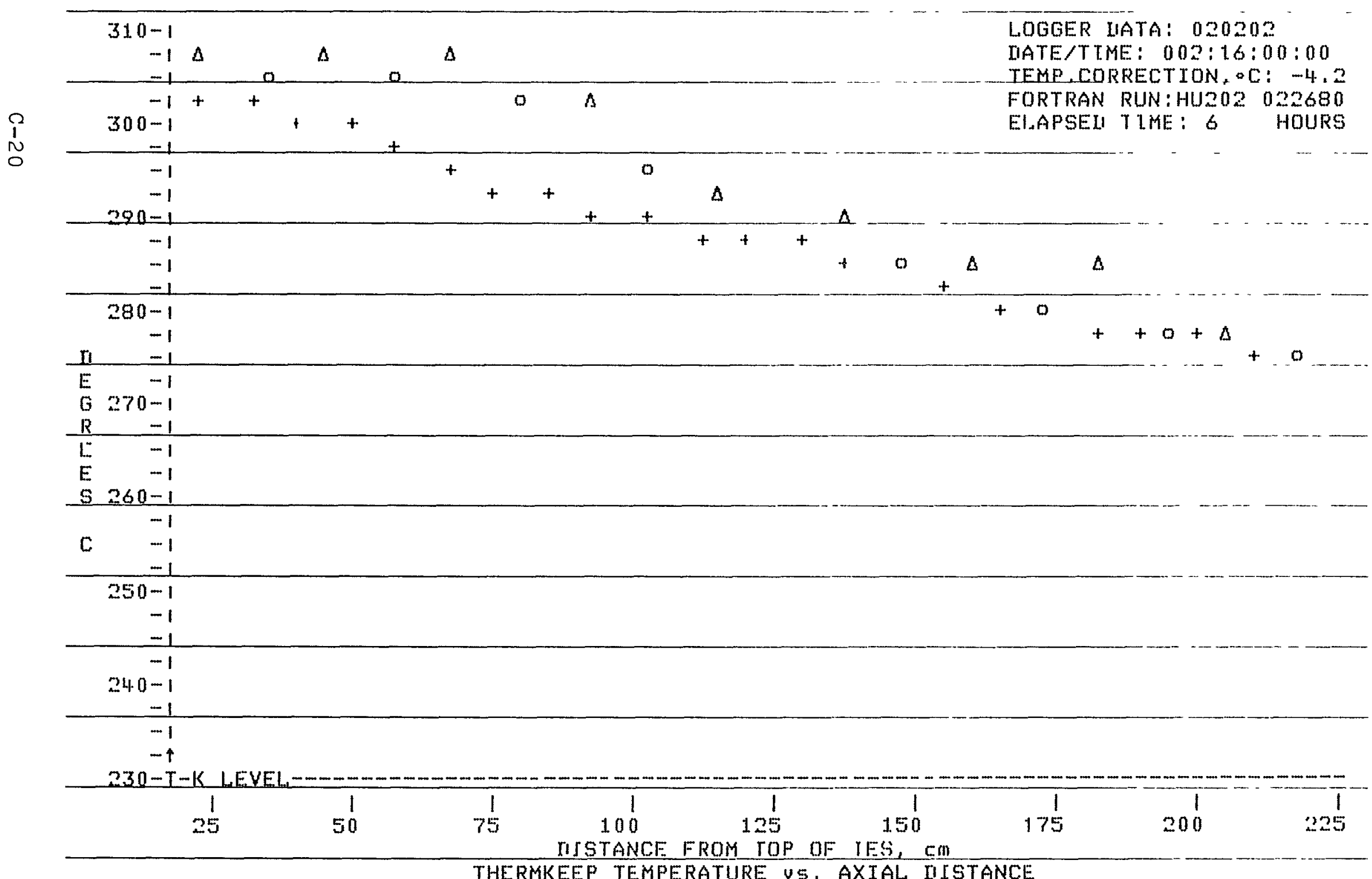

Fıgure C-18. Thermkeep temperature profile, charge test 202 (3,3 gpm L after 6 hours. 


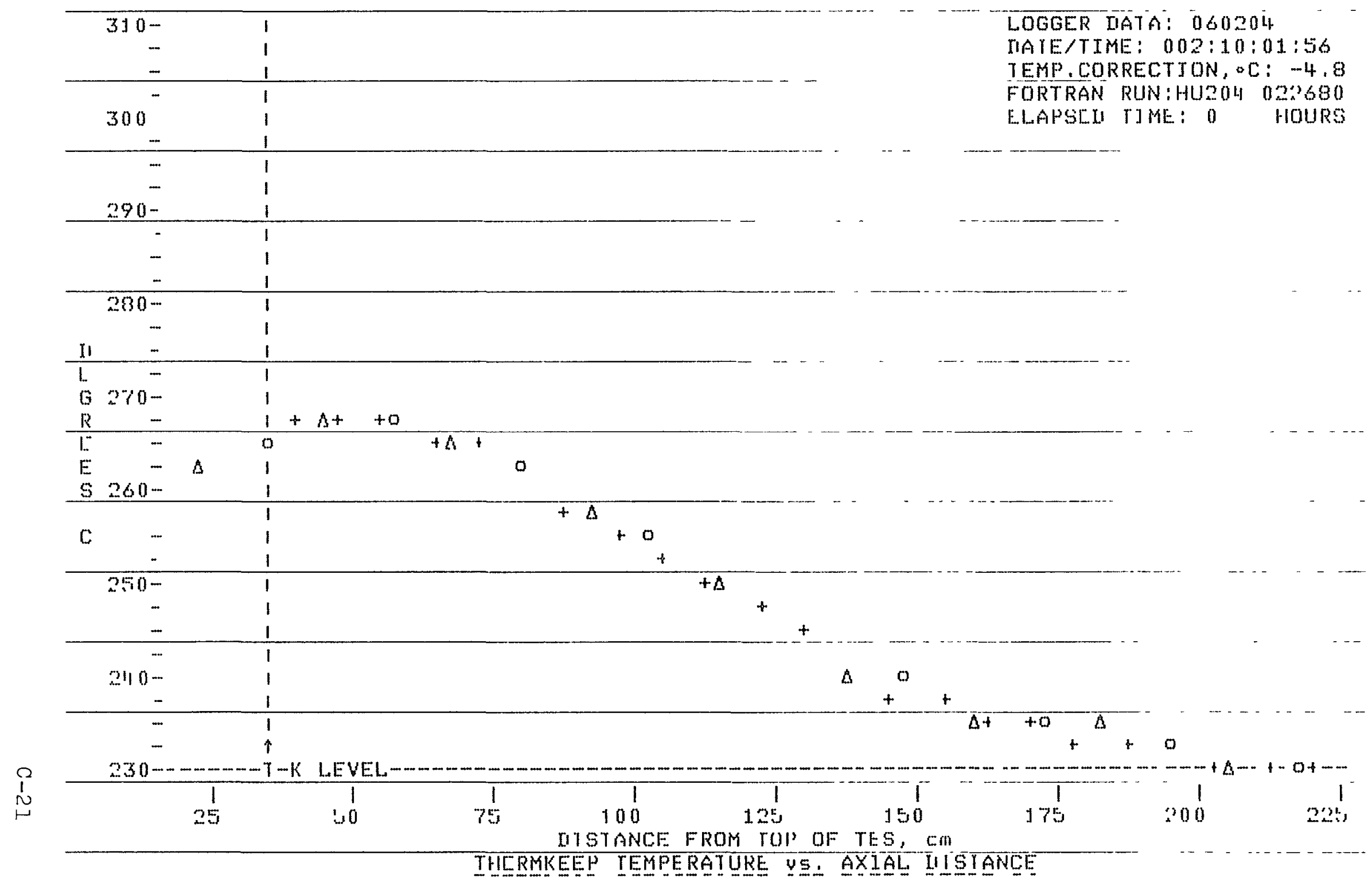

Figure C-19. Thermkeep temperature profile, charge test 204 ( $3.7 \mathrm{gpm}$ ), startıng profile. 


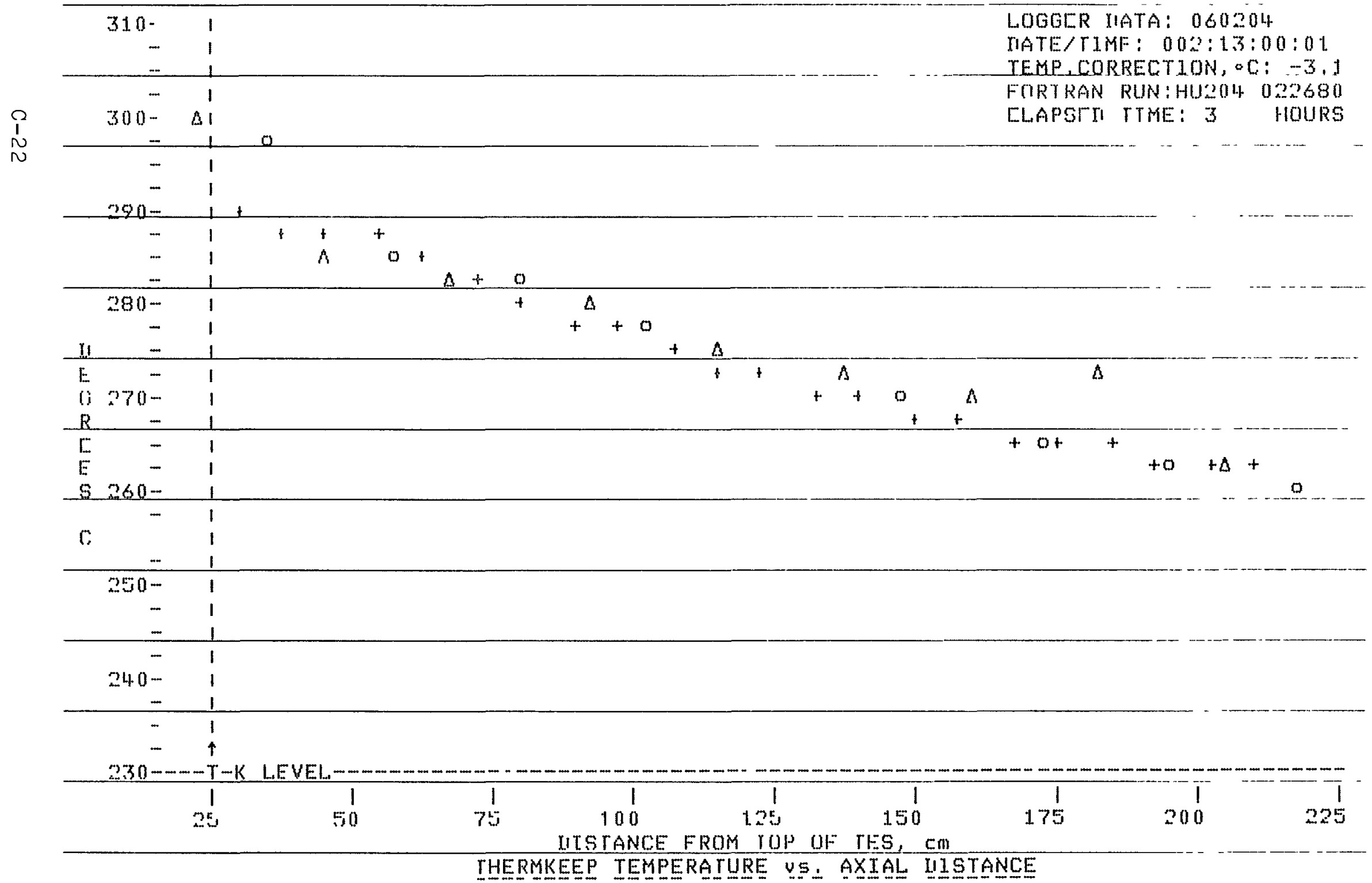

Figure C-20. Thermkeep temperature profile, charge test 204 (3,3 gpmL, after 3 hours. 


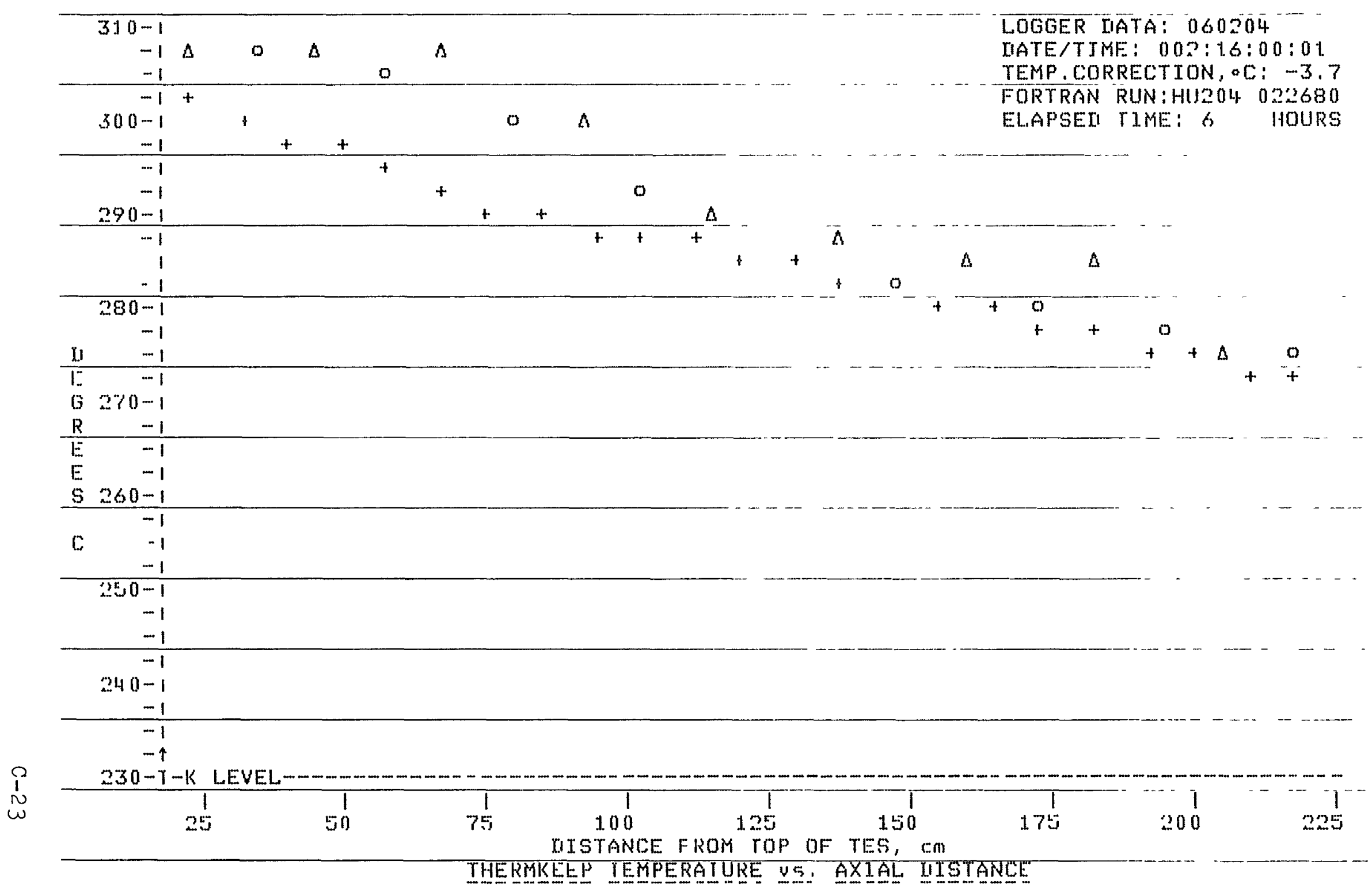

Figure C-21. Thermkeep temperature profile, charge test 204 ( $3.7 \mathrm{gpm}$ ), after 6 hours. 


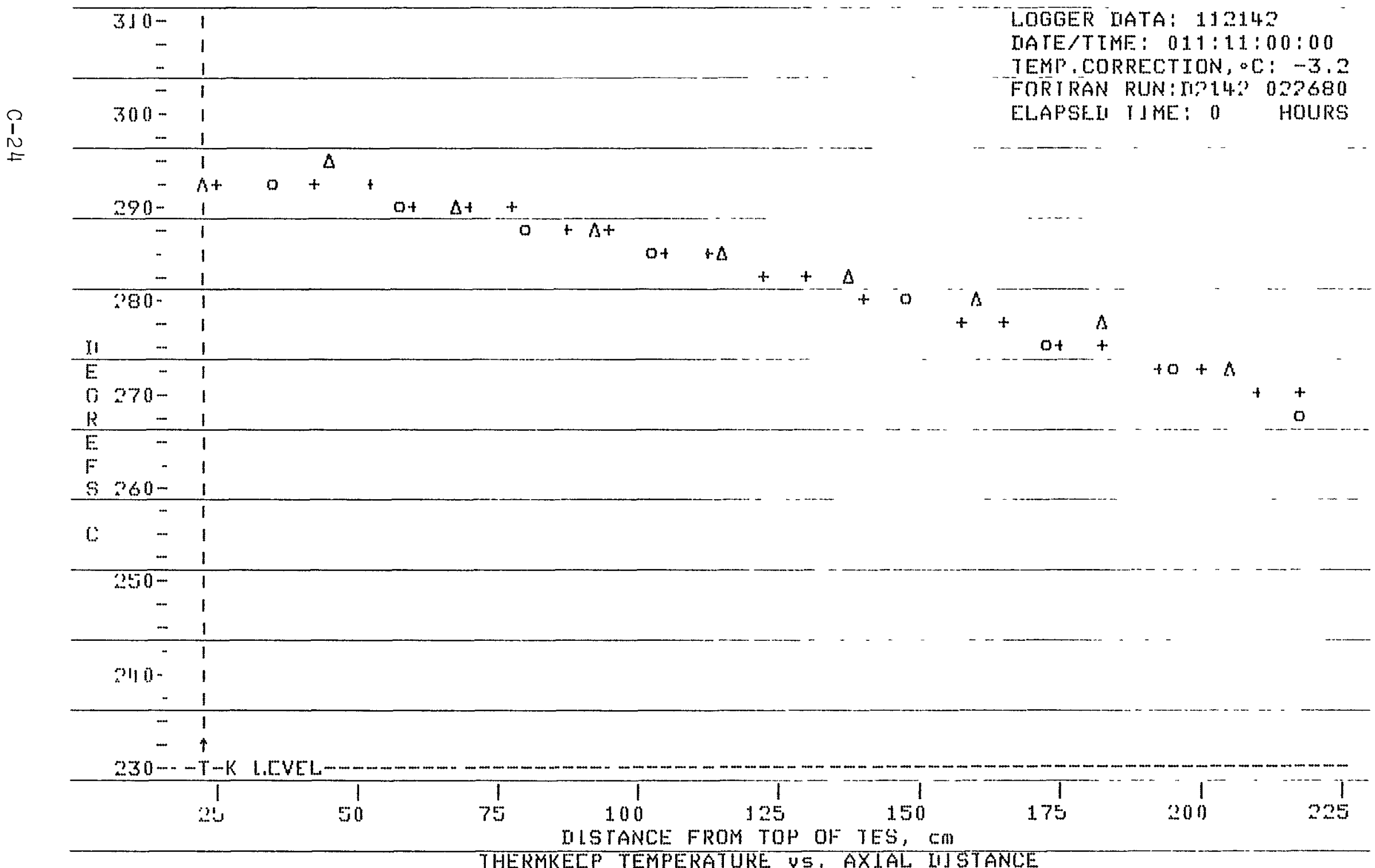

Figure C-22. Thermkeep temperature profile, discharge test 214-3 (1,1 gpm), starting profile. 


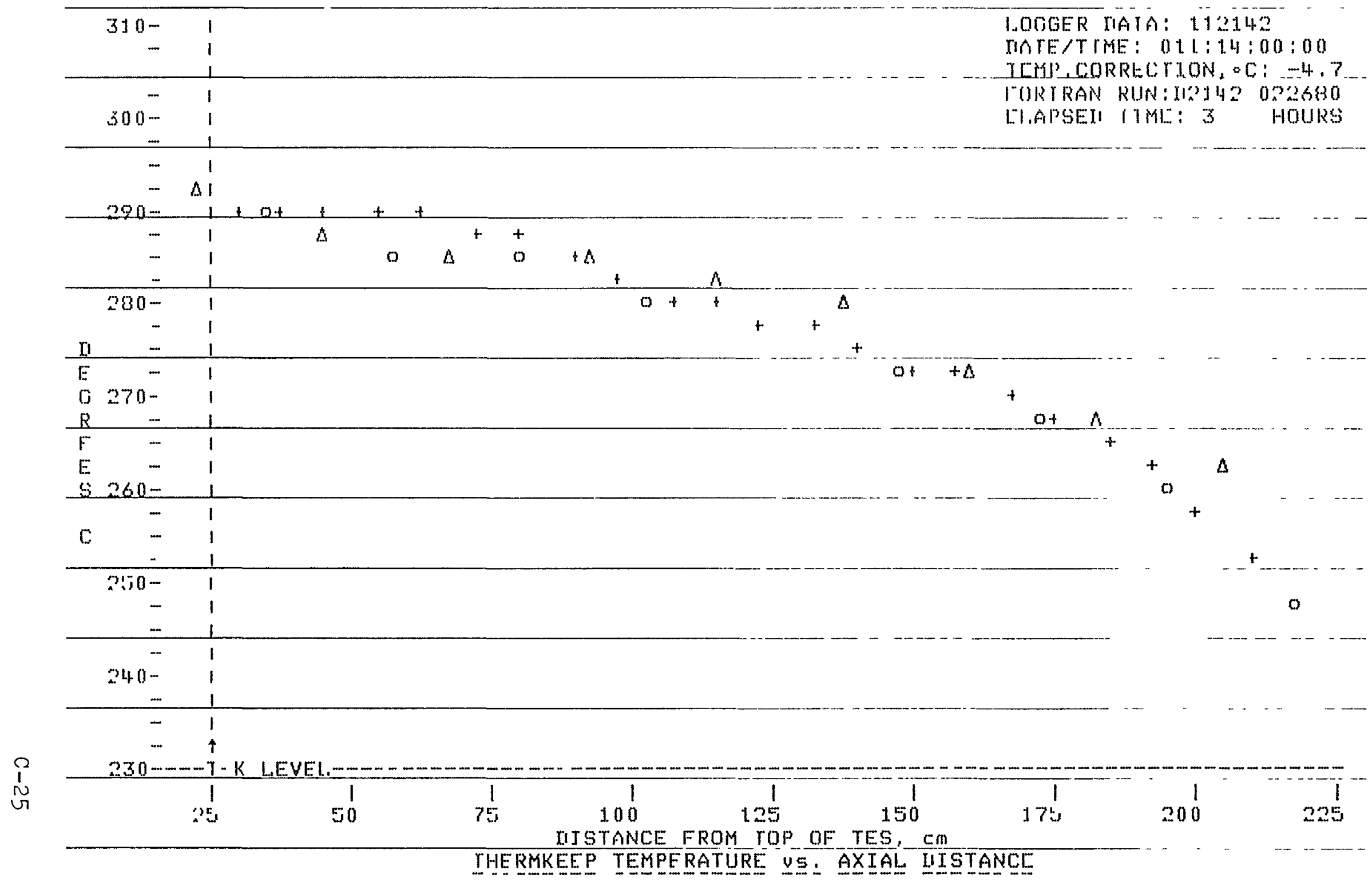

Figure C-23. Thermkeep temperature profile, discharge test 214-3 ( 1.1 gpm), after 3 hours. 


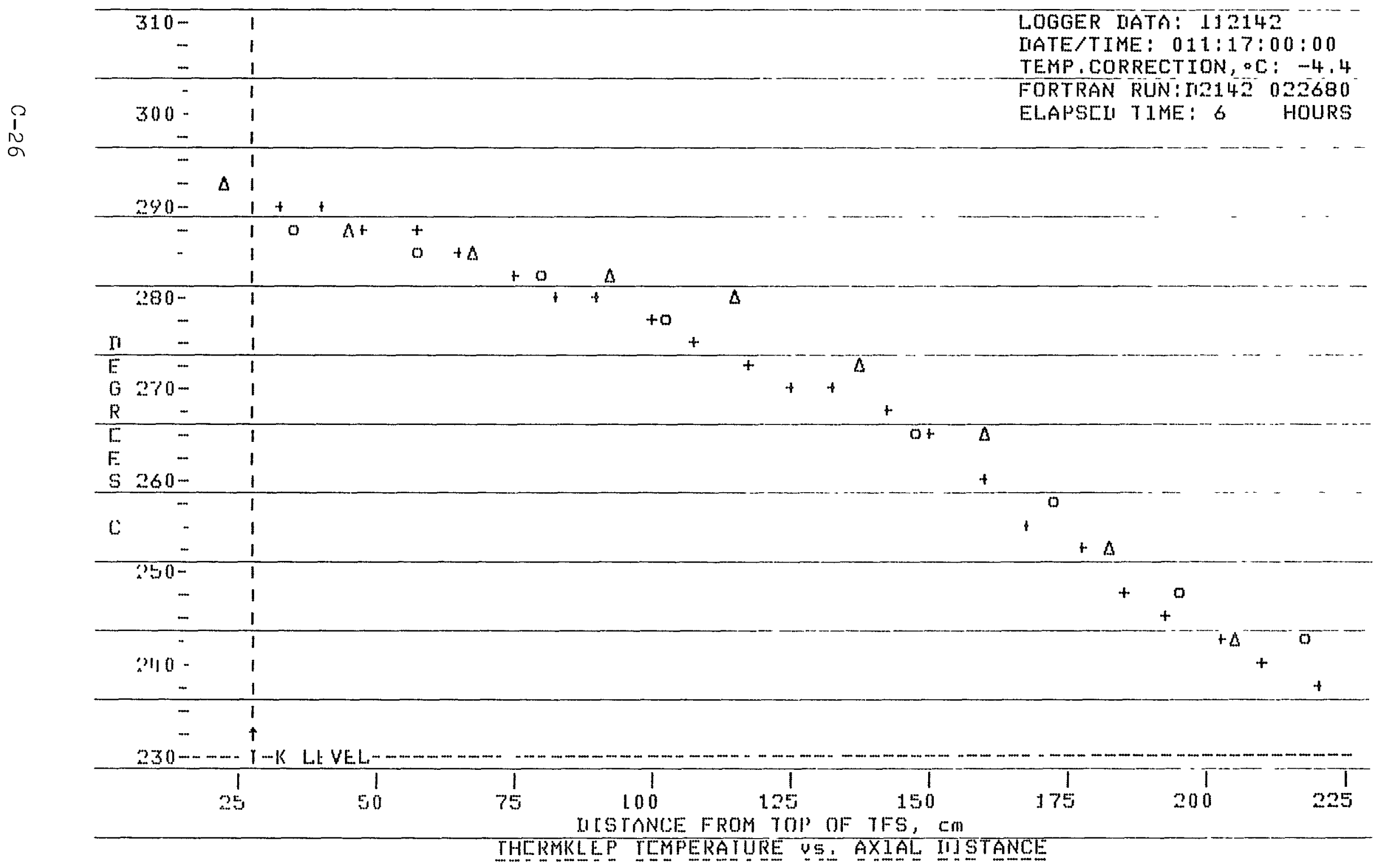

Figure C-24. Thermkeep temperature profile, discharge test 214-3 (I,I gpm) after 6 hours, 


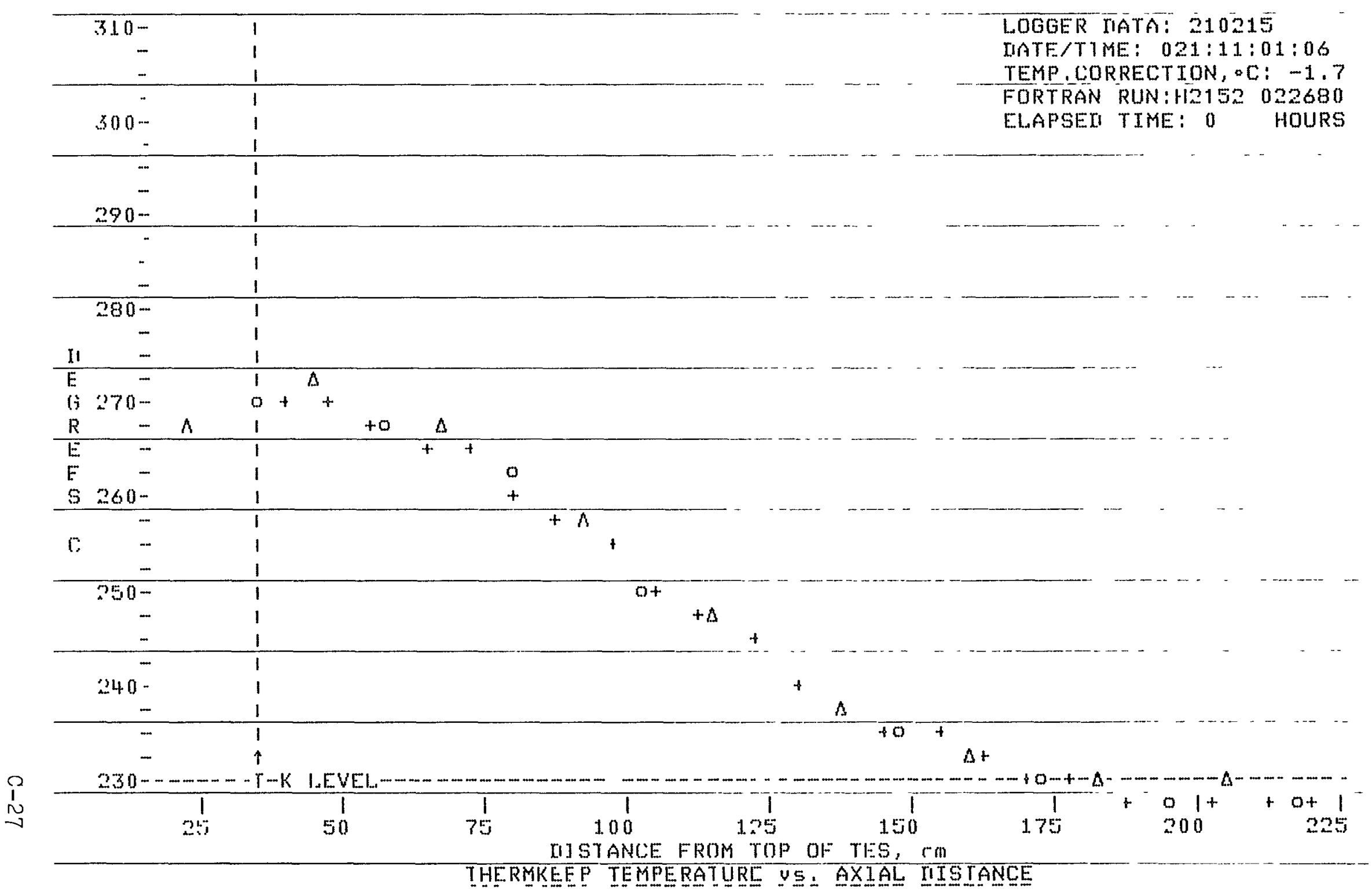

Figure C-25. Thermkeep temperature profile, charge test 215-2 ( $1.1 \mathrm{gpm})$, starting profile. 


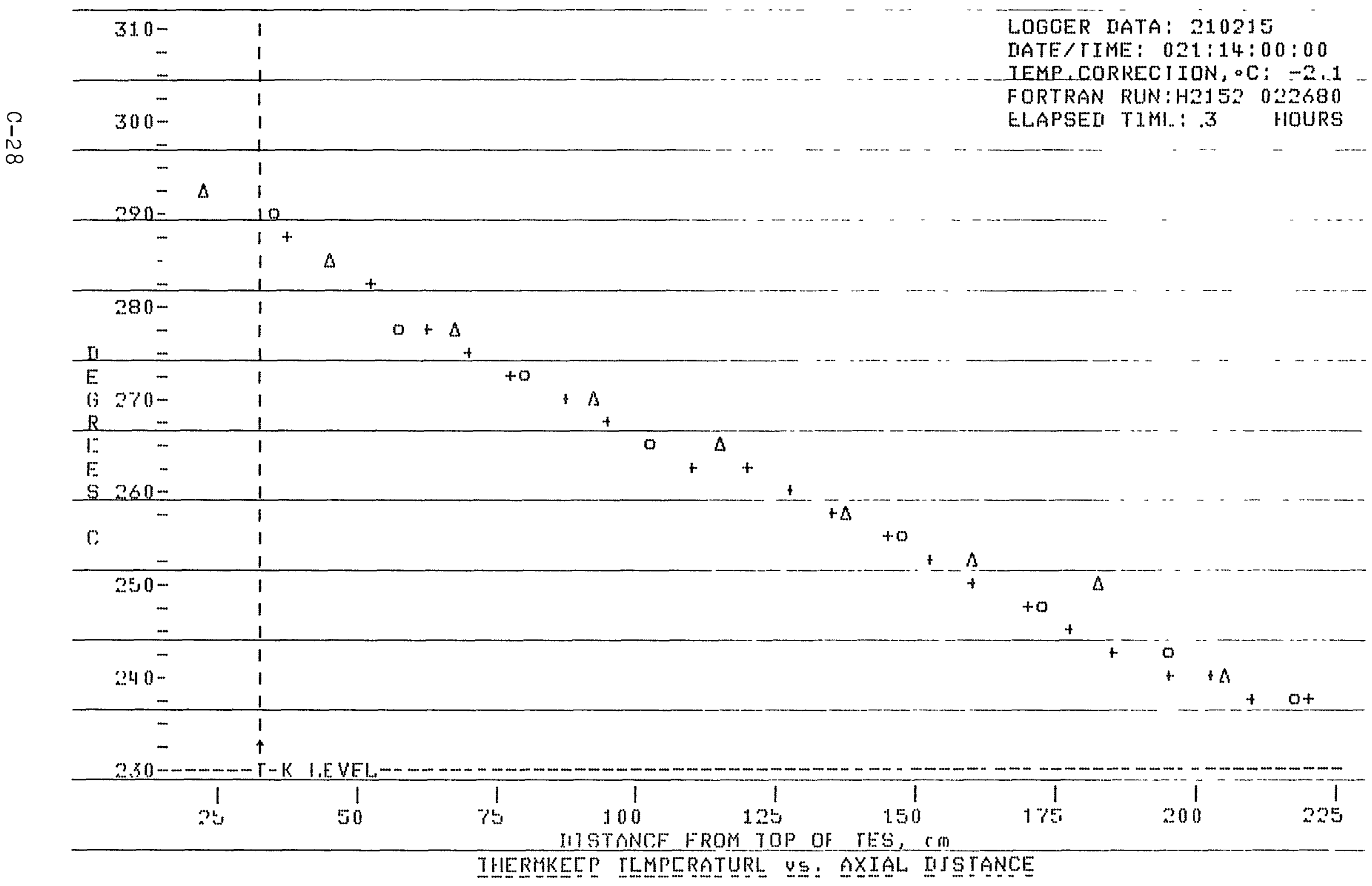

Figure C-26. Thermkeep temperature profile, charge test 215-2 (I,I gpm), after 3 hours. 


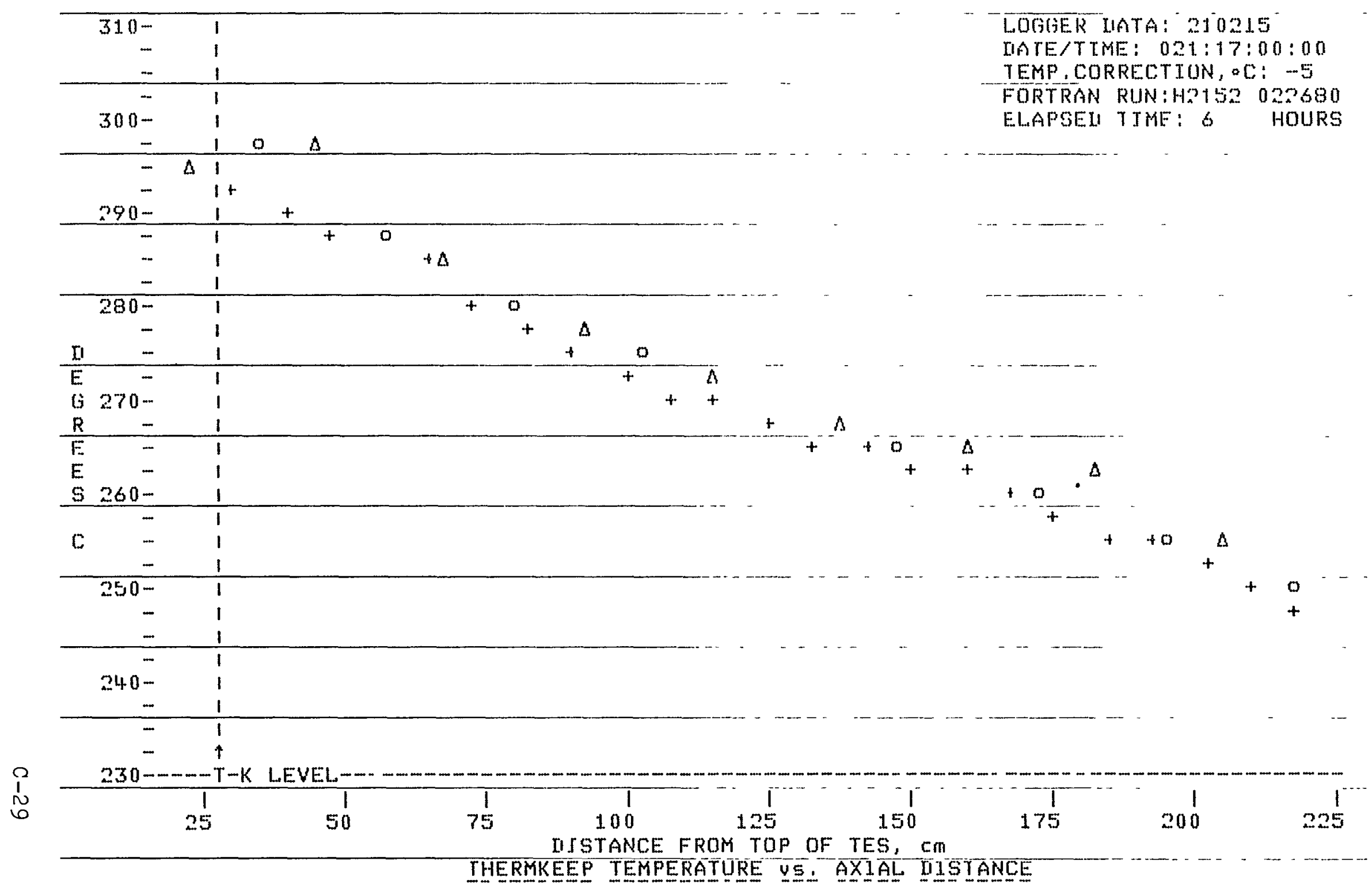

Figure C-27. Thermkeep temperature profile, charge test 215-2 ( 1.1 gpm), after 6 hours . 


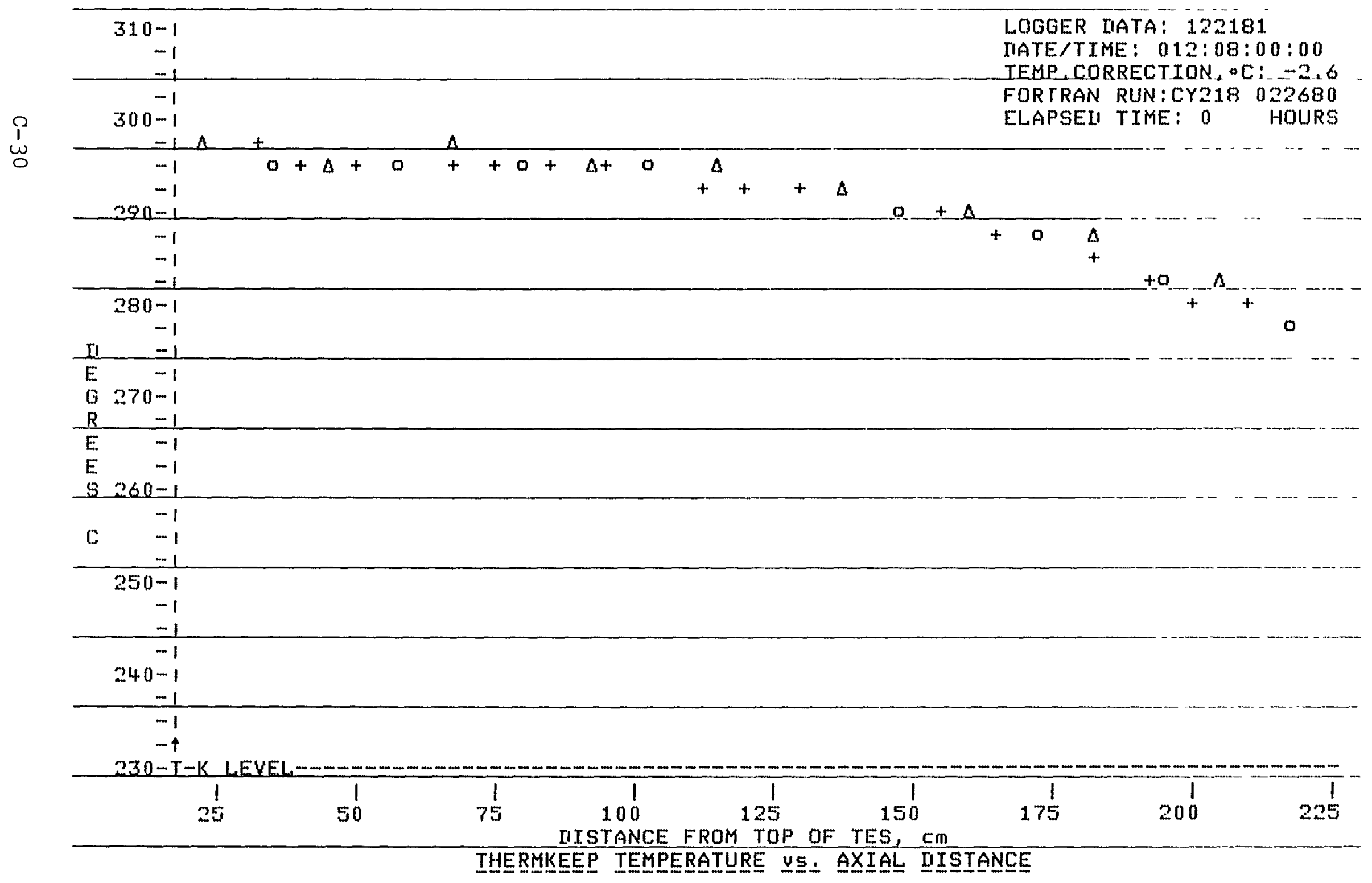

Figure C-28. Thermkeep temperature profile, cyclic test 218 (1,0 gpm L, starting profile. 


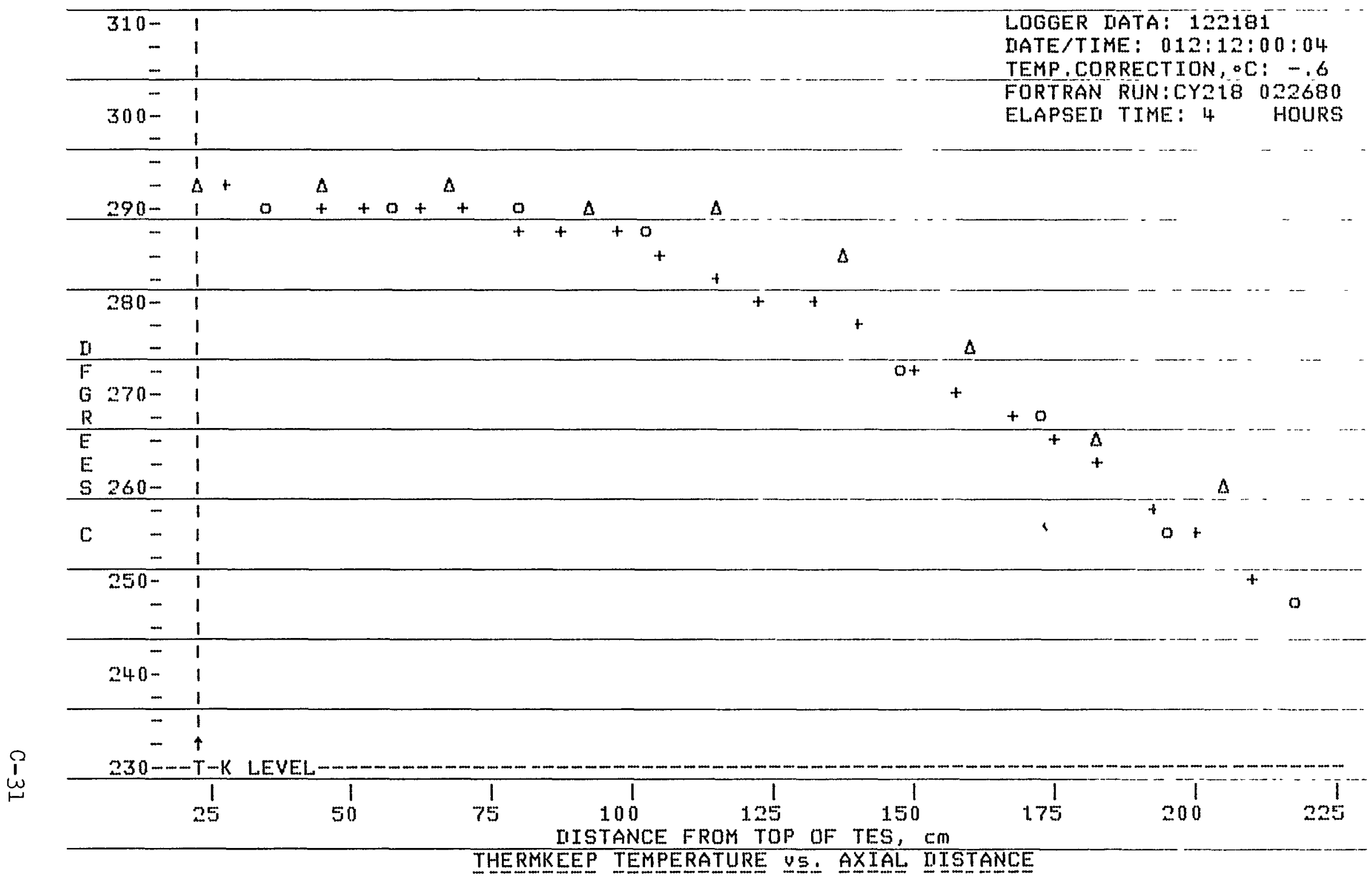

Figure C-29. Thermkeep temperature profile, cyclic test 218 ( $1.9 \mathrm{gpm}$, at end of first discharge phase. 


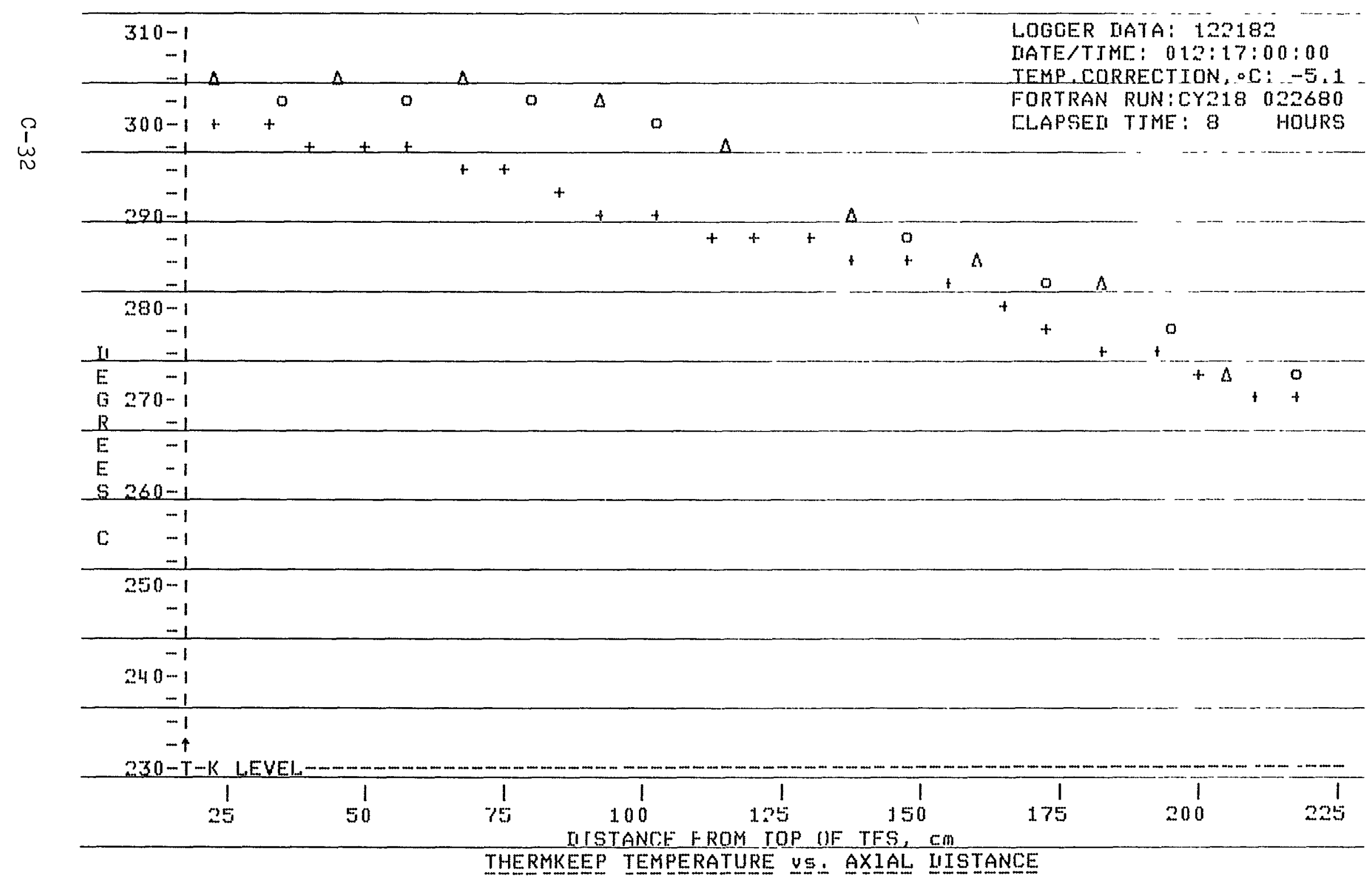

Figure C-30. Thermkeep temperature profile, cyclic test 218 ( $1.2 \mathrm{gpm}$ ), at end of first charge phase, 


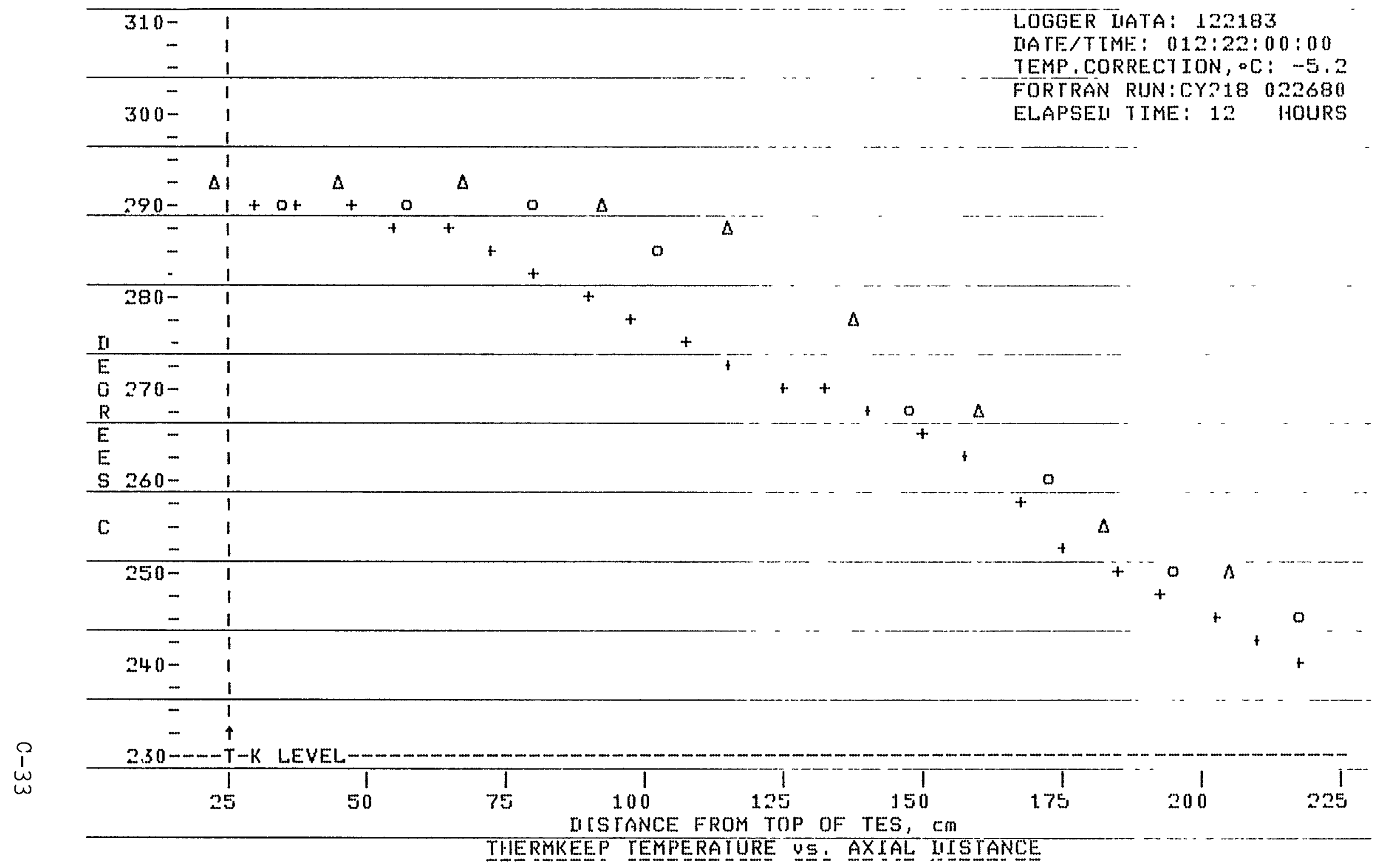

Figure C-3l. Thermkeep temperature profile, cyclic test 218 ( 1.9 gram), at end of second discharge phase. 


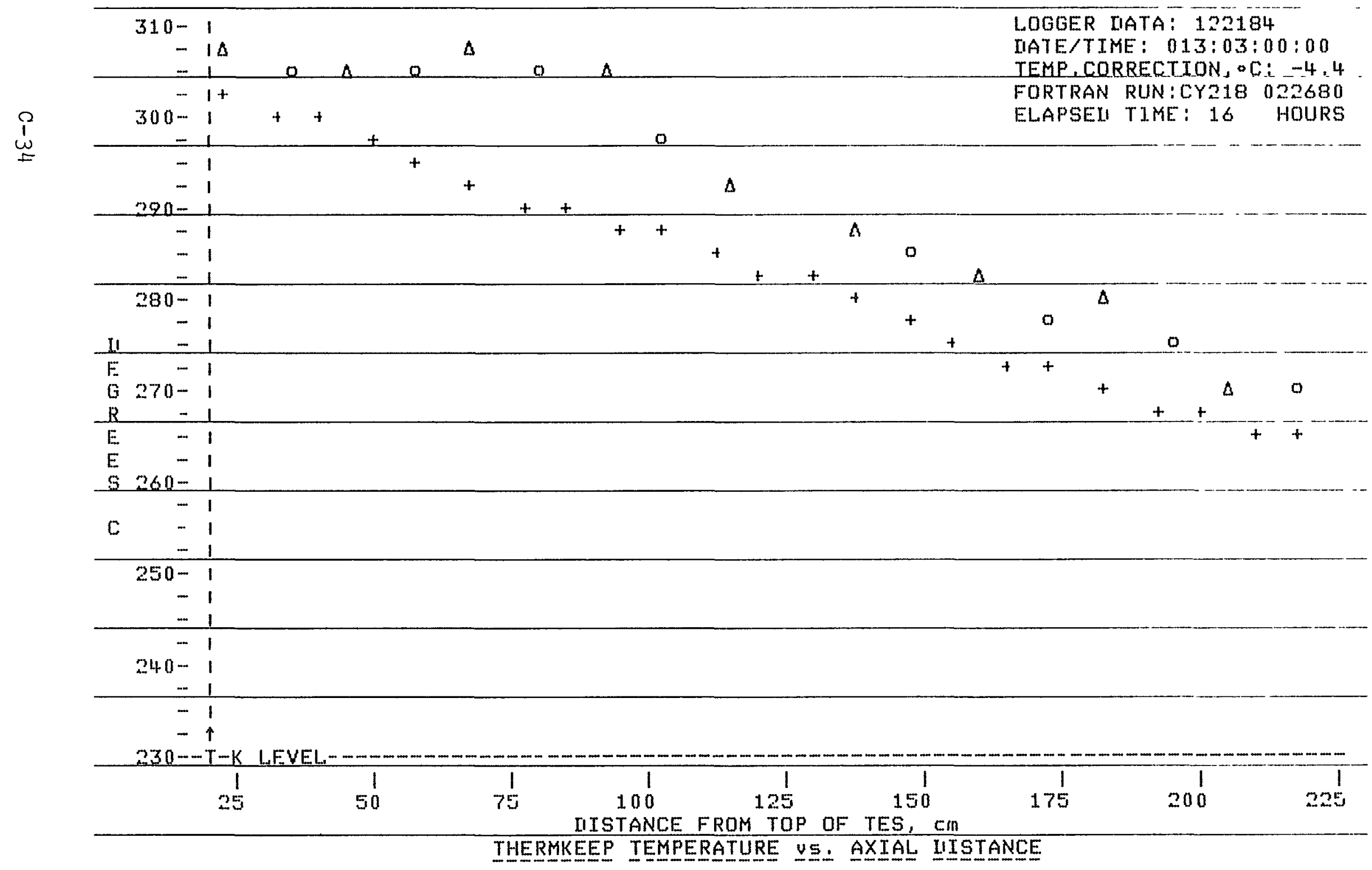

Figure C-32, Thermkeep temperature profile, cyclic test 218 ( $1.9 \mathrm{gpm})$, at end of second charge phase. 


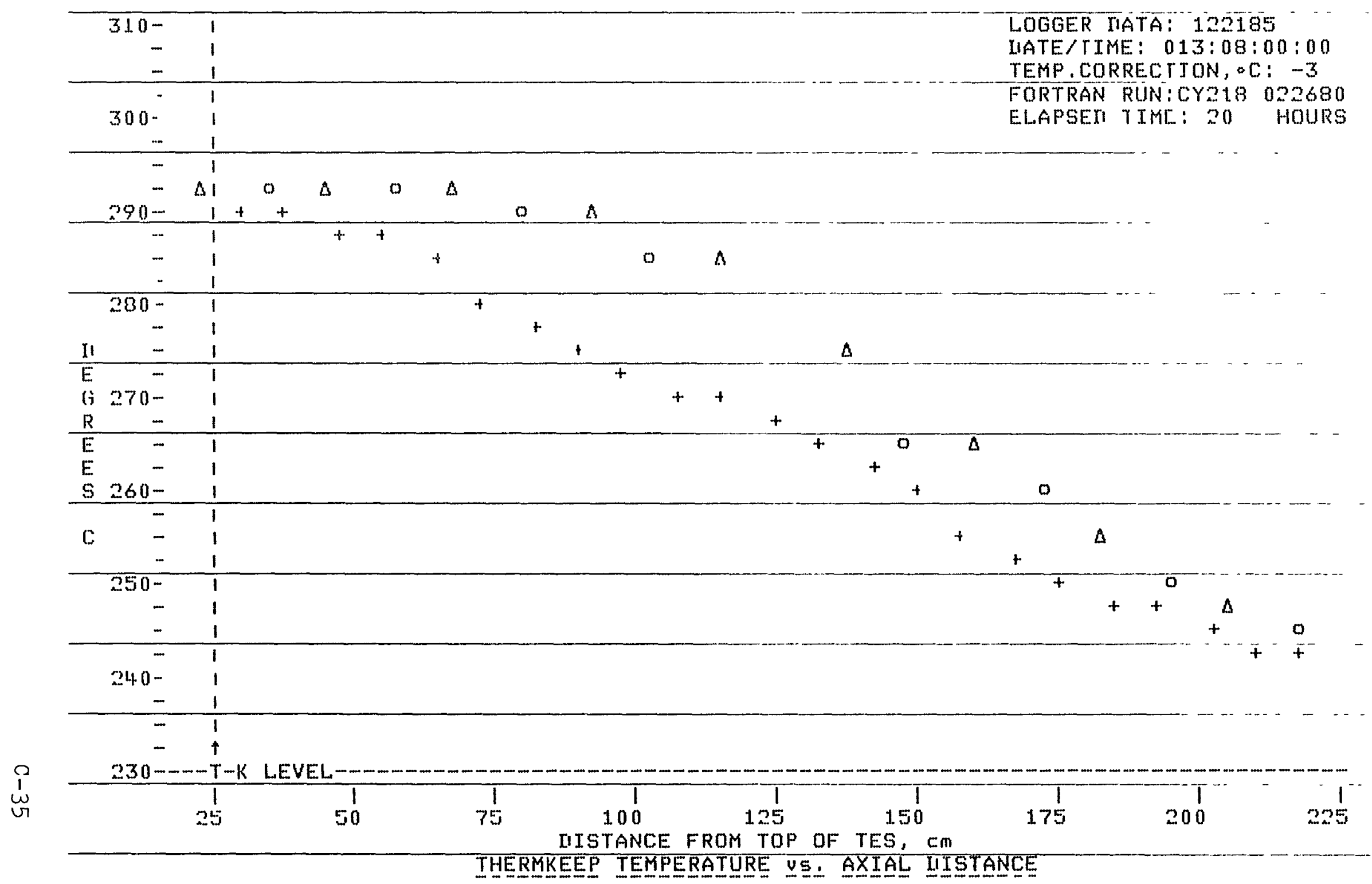

Figure C-33. Thermkeep temperature profile, cyclic test 218 ( $1.9 \mathrm{gpm}$ ), at end of third discharge phase. 


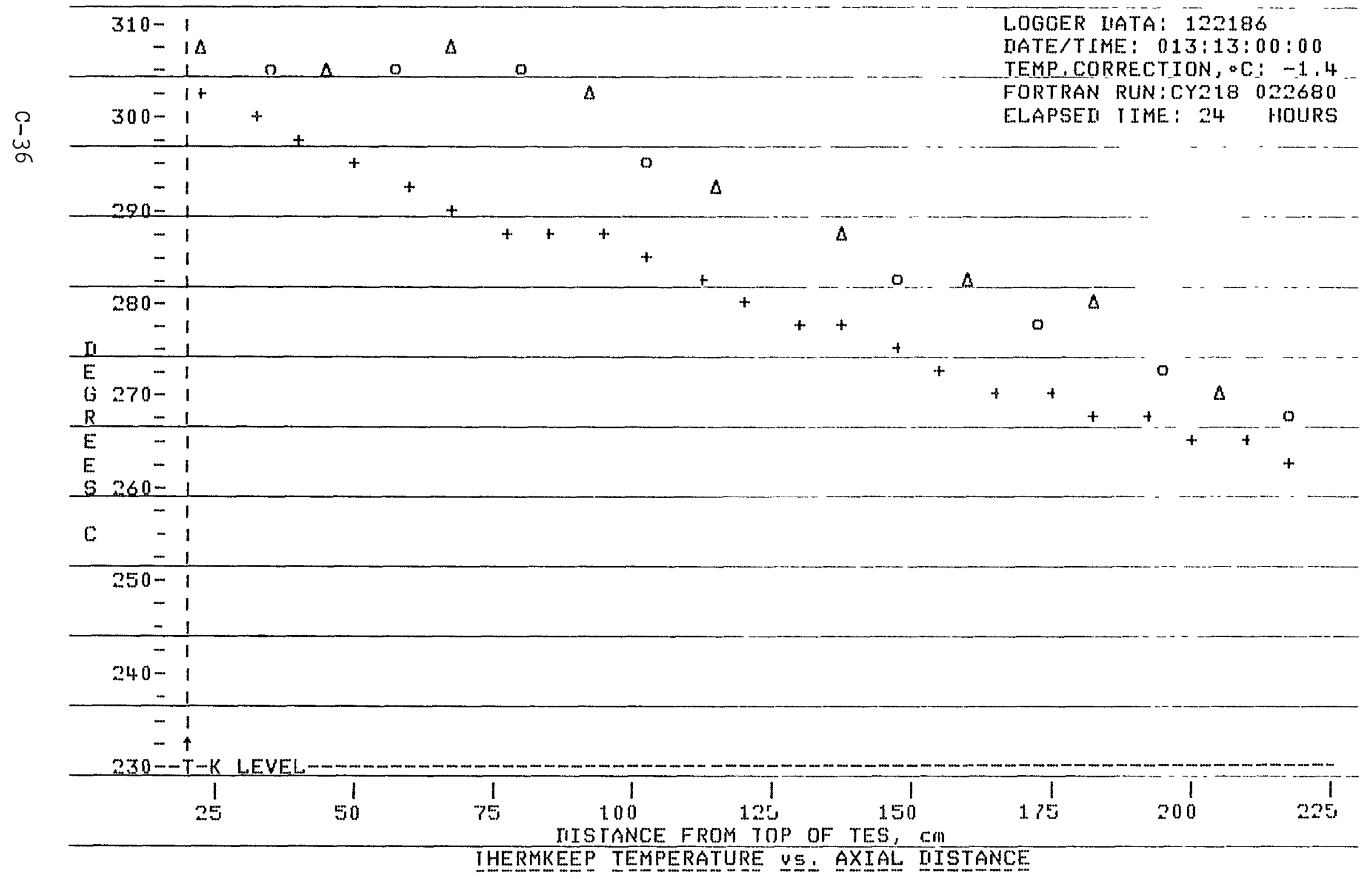

Figure C-34. Thermkeep temperature profile, cyclic test 218 ( $1.2 \mathrm{gpm} 2$, at end of third charge phase, 


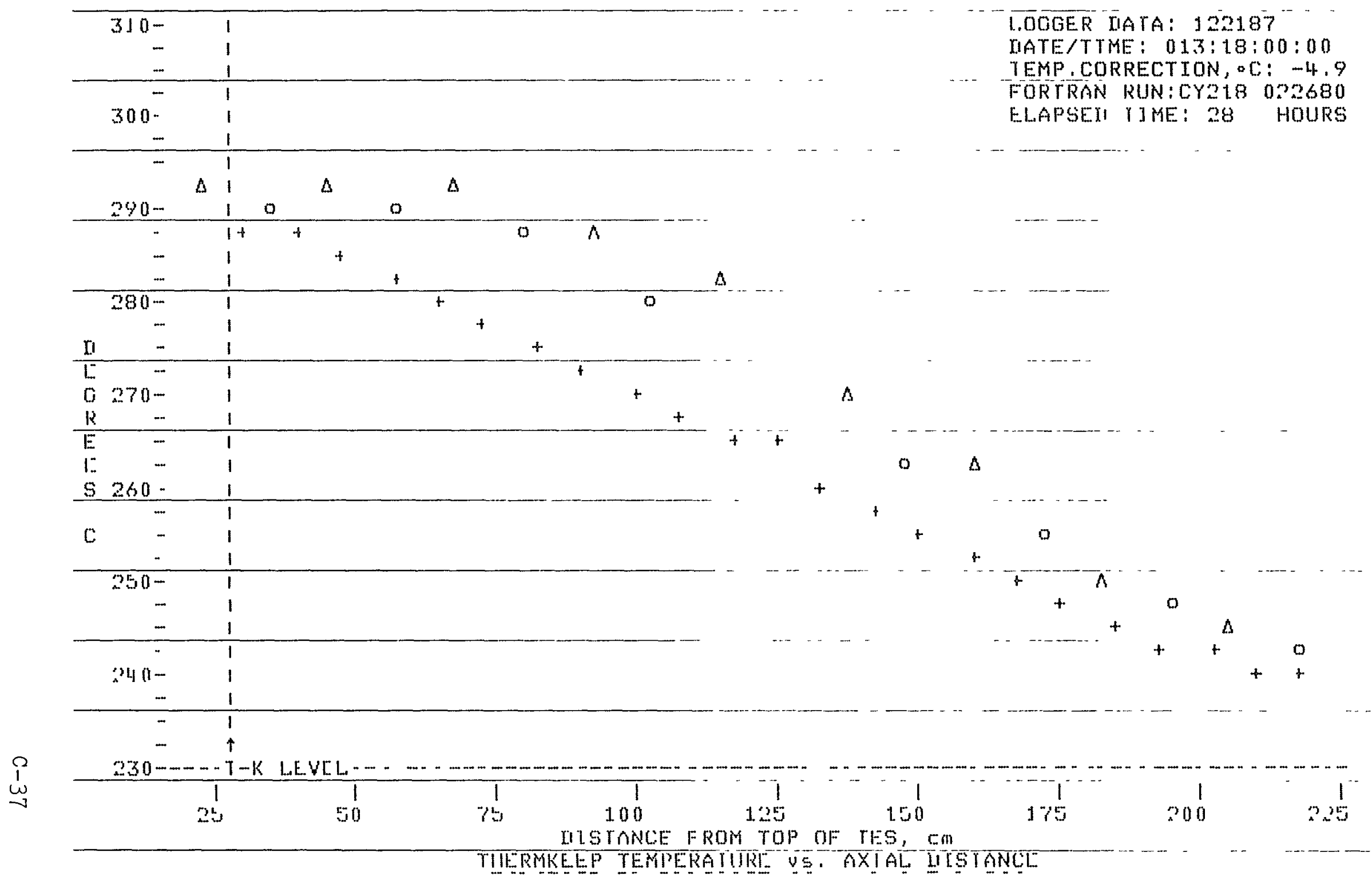

Figure C-35. Thermkeep temperature profile, cyclic test 218 ( $1.9 \mathrm{gpm}$ ), at end of fourth discharge phase. 


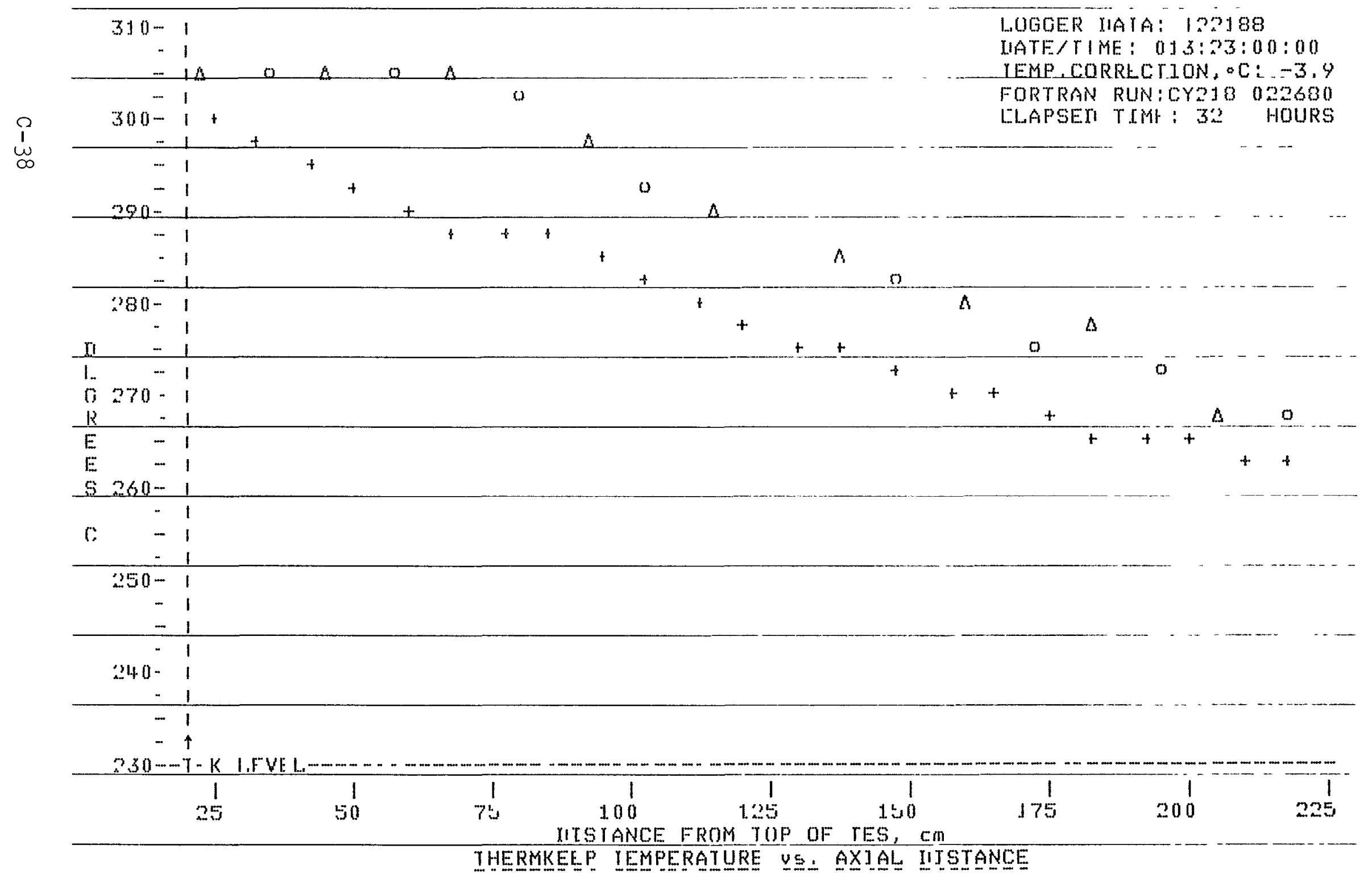

Figure C-36. Thermkeep temperature profile, cyclic test 218 (1.2 gpm), at end of fourth charge phase, 


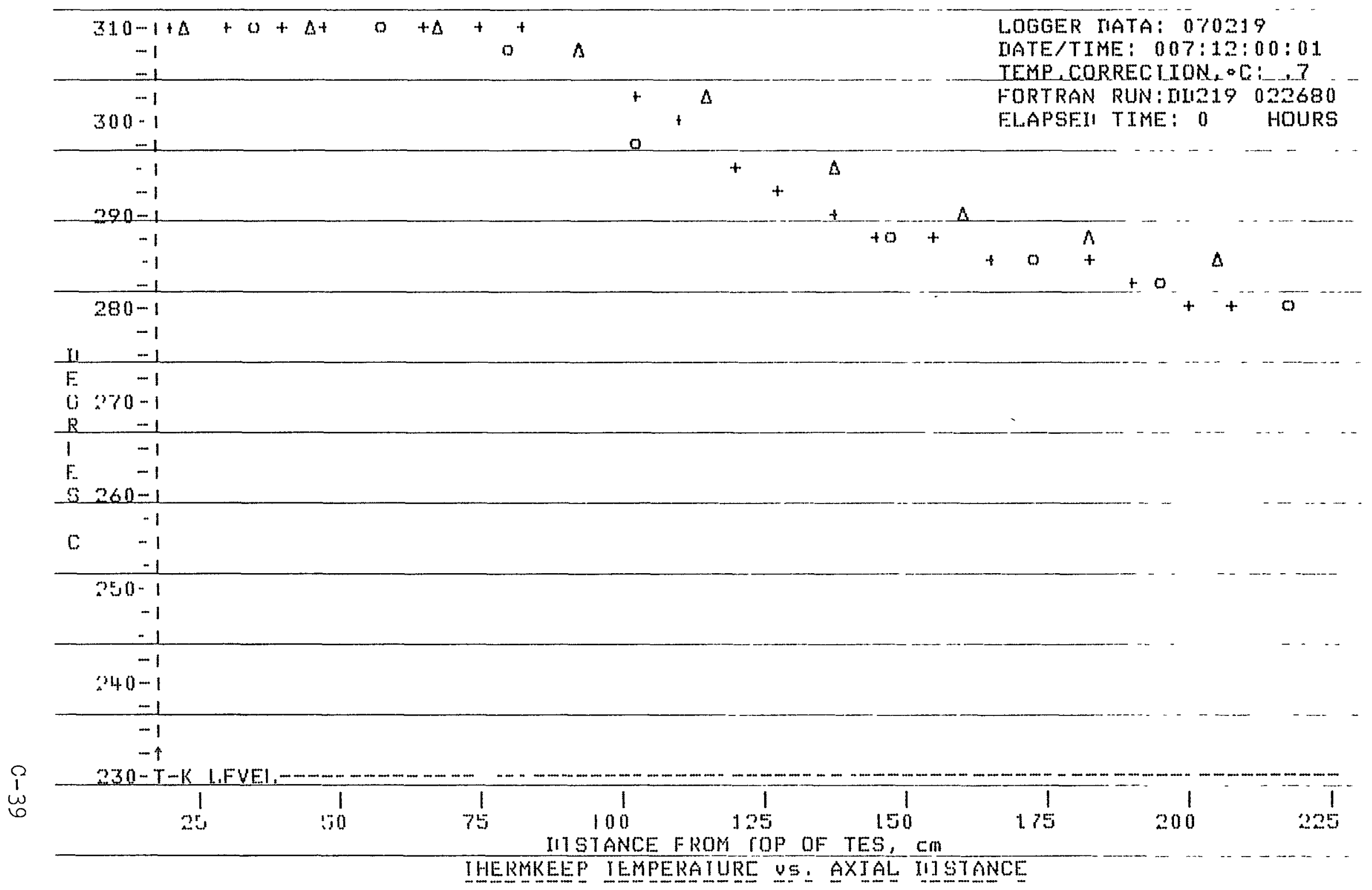

Figure C-37. Thermkeep temperature profile, discharge test 219 ( $7.7 \mathrm{gpm})$, startıng profile. 


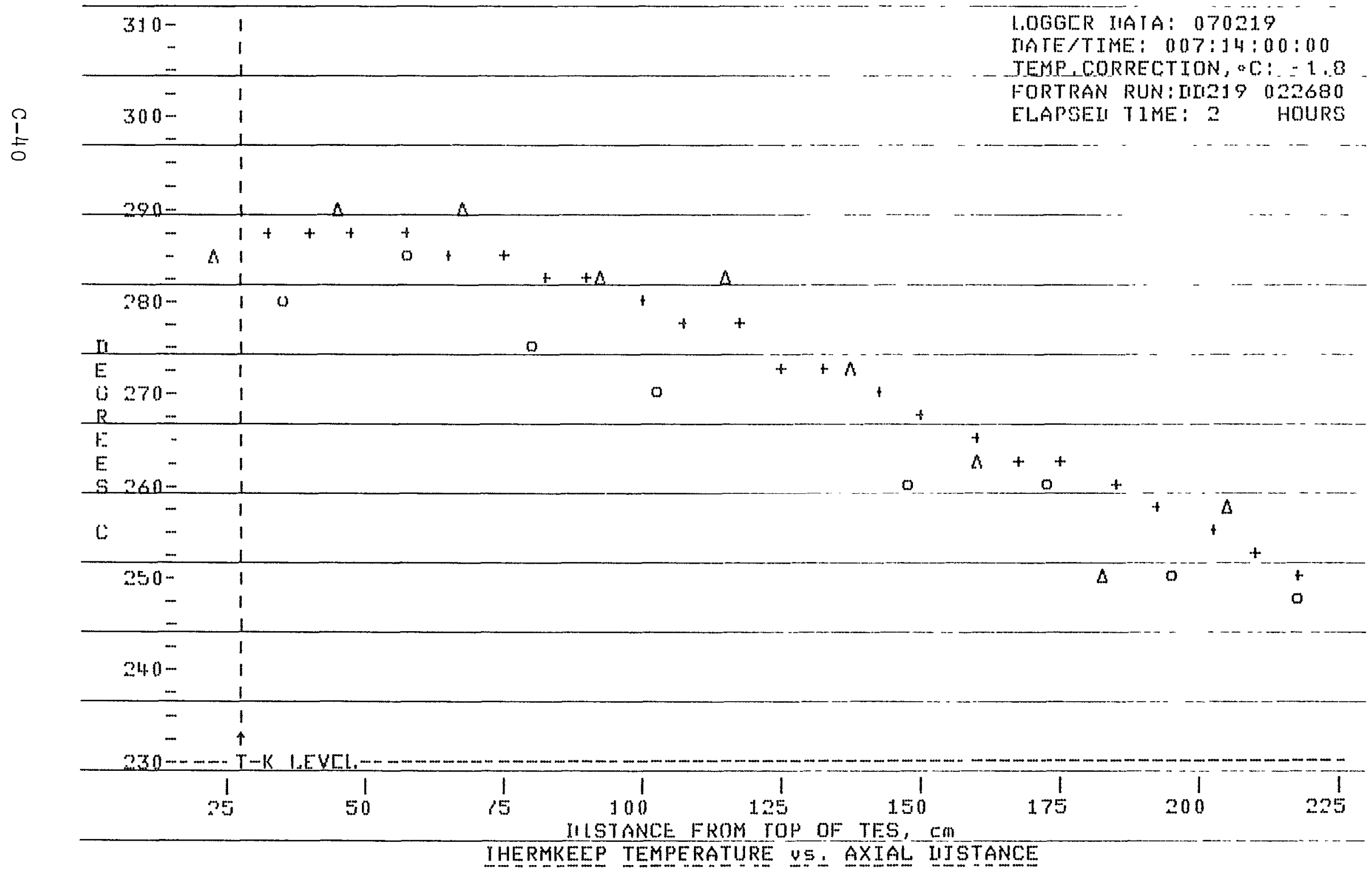

Figure C-38. Thermkeep temperature proflle, discharge test 212 ( $7.7 \mathrm{gpm})$, after 2 hours. 


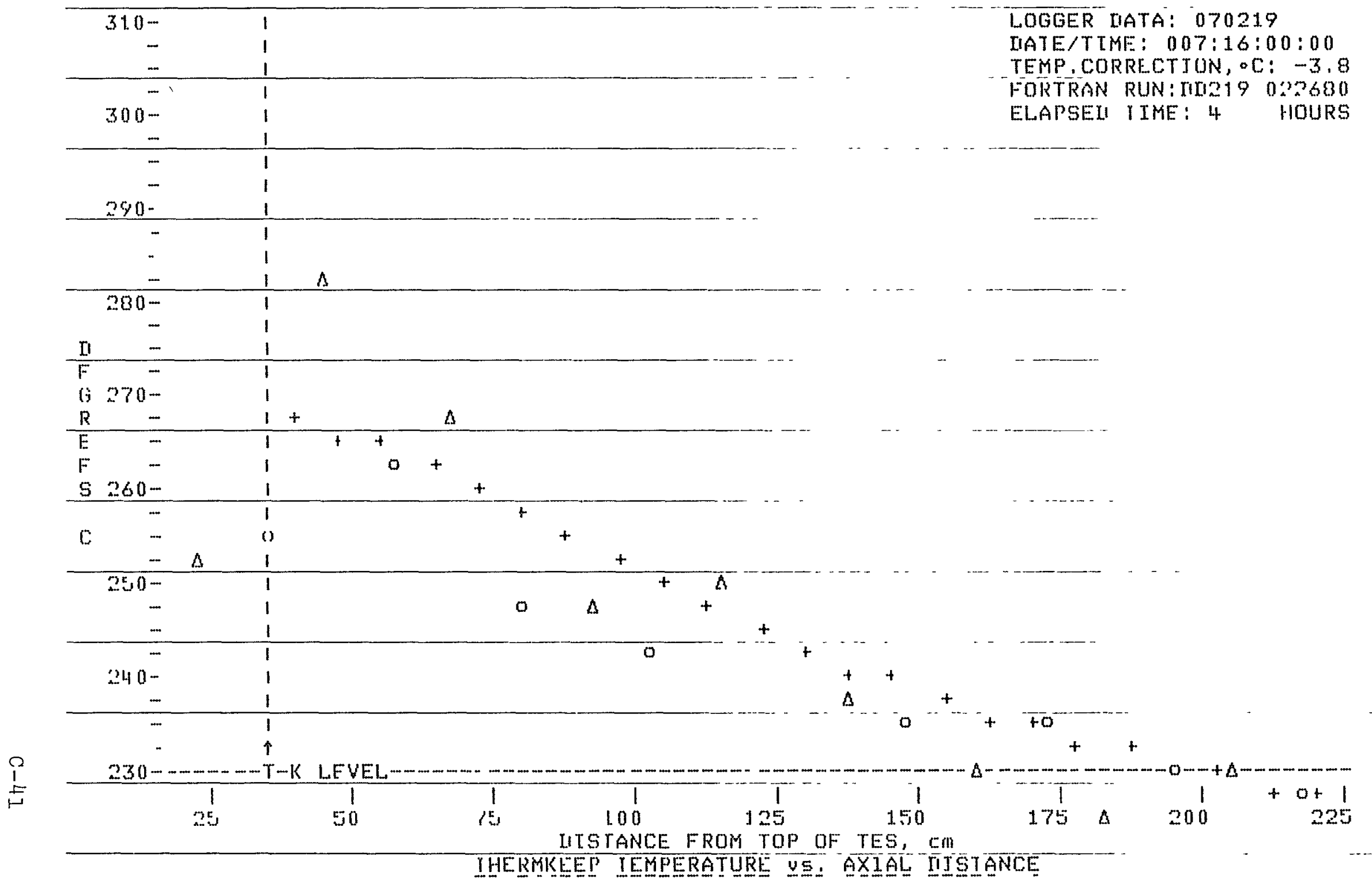

Fıgure C-39. Thermkeep temperature profile, discharge test 219 (7.7 gpm), after 4 hours . 


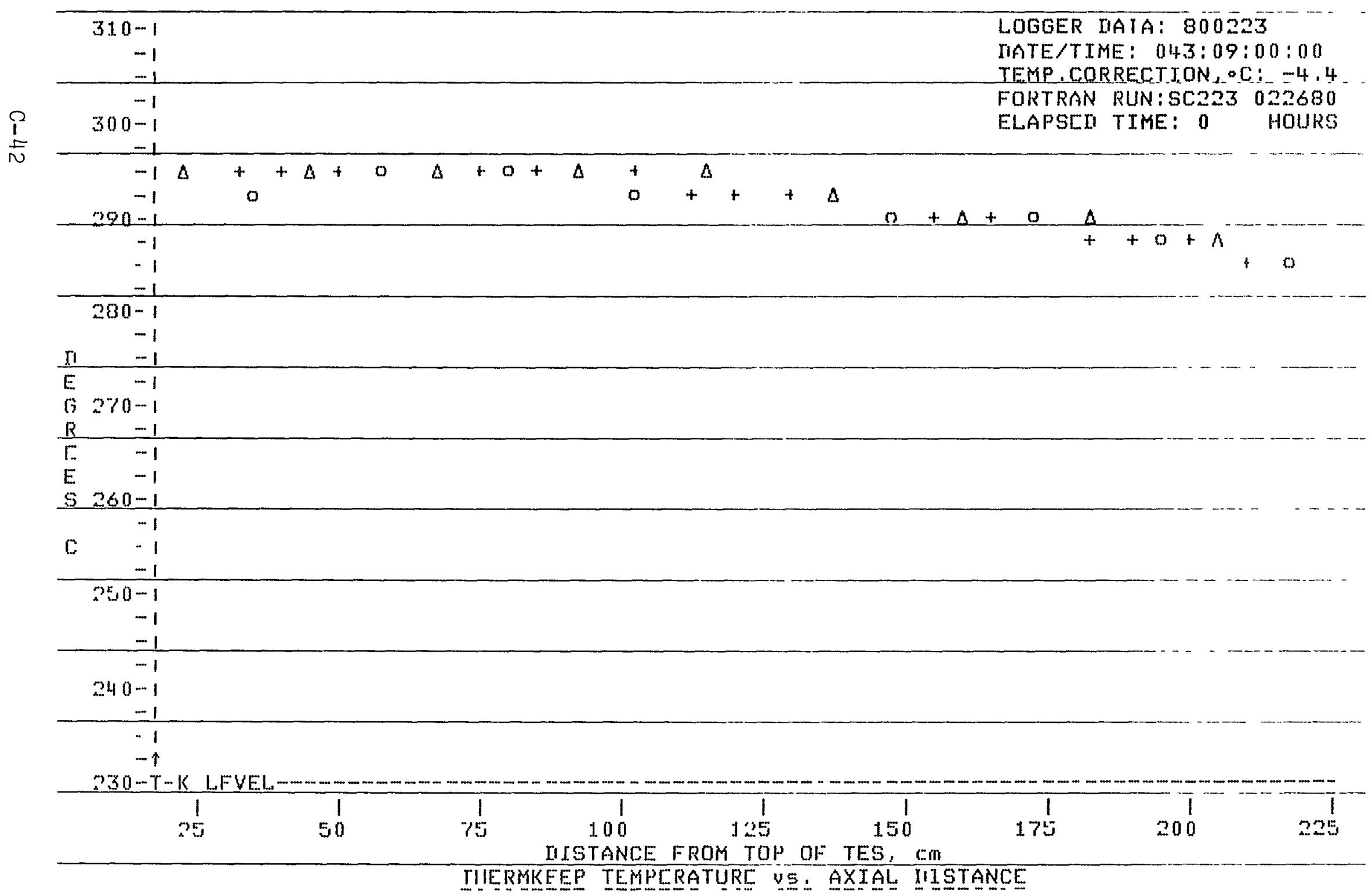

Figure C-40. Thermkeep temperature profile, solar cycle test 223, starting profile, cycle 1. 


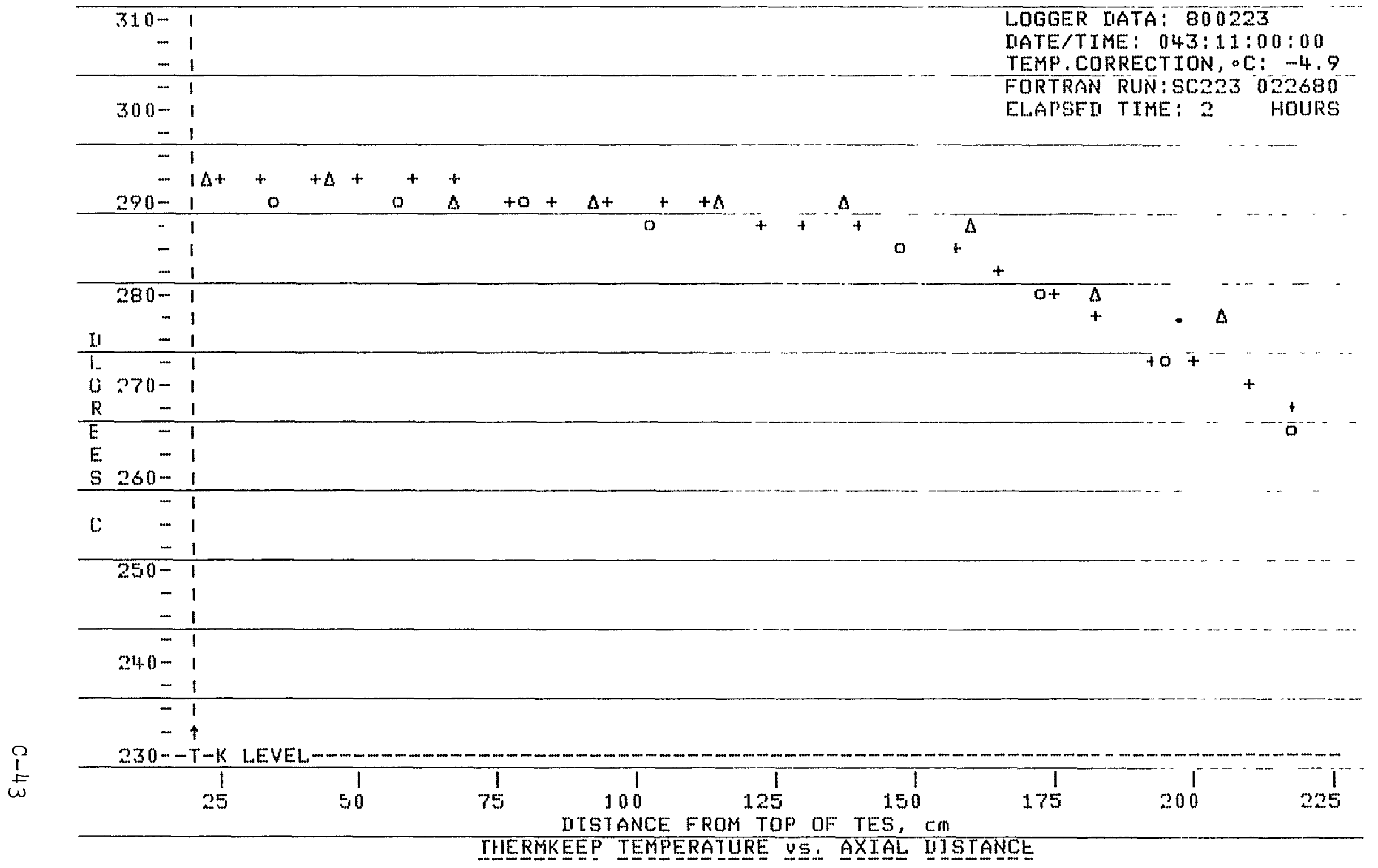

Figure C-41. Thermkeep temperature profile, solar cycle test 223, at end of Phase 1 discharge, cycle 1. 


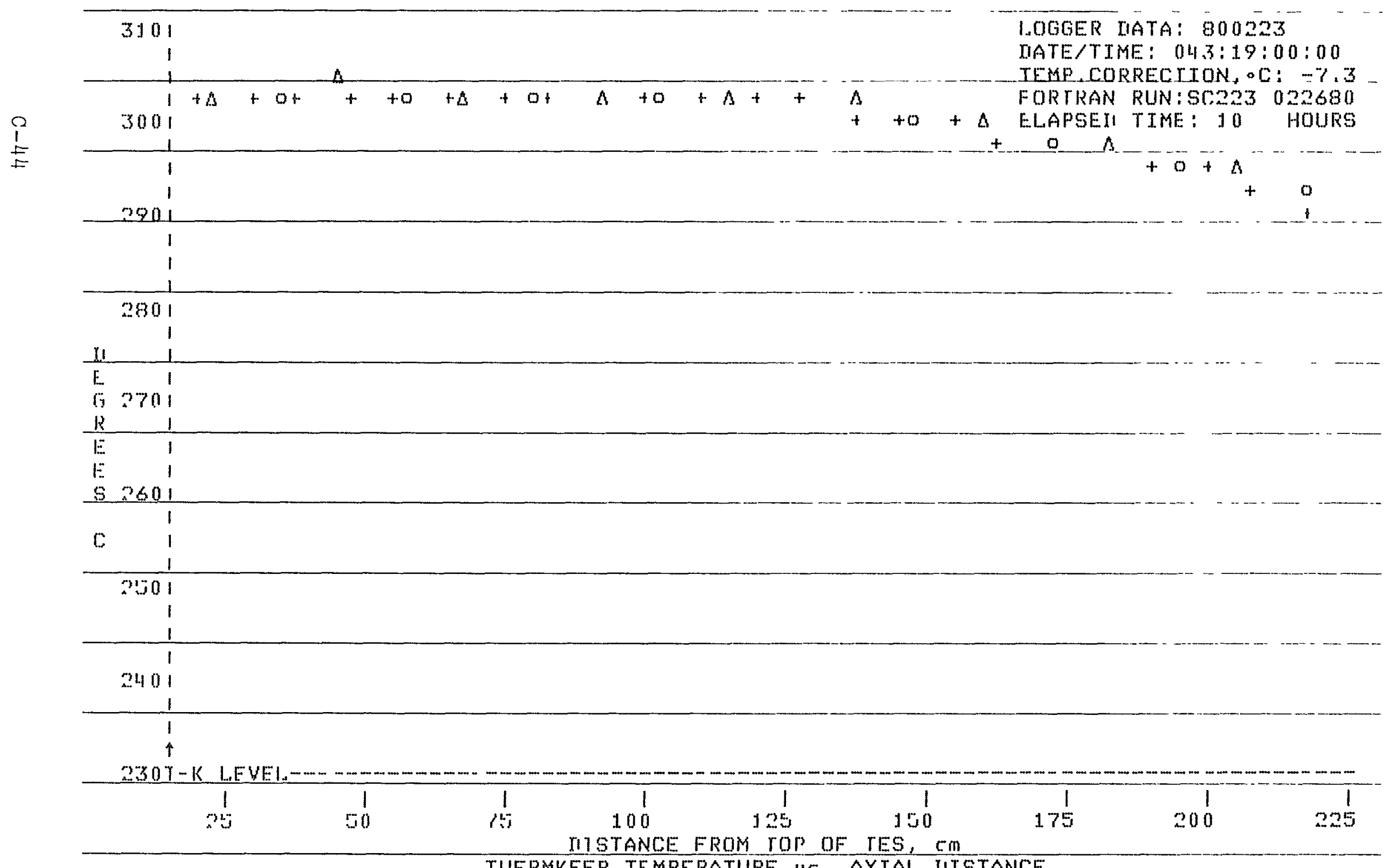

TIIERMKEEP IEMPERATURE QS. AXIAL IIISTANCE

Figure C-42. Thermkeep temperature profıle, solar cycle test 223, at end of Cycle 1 , charge. 


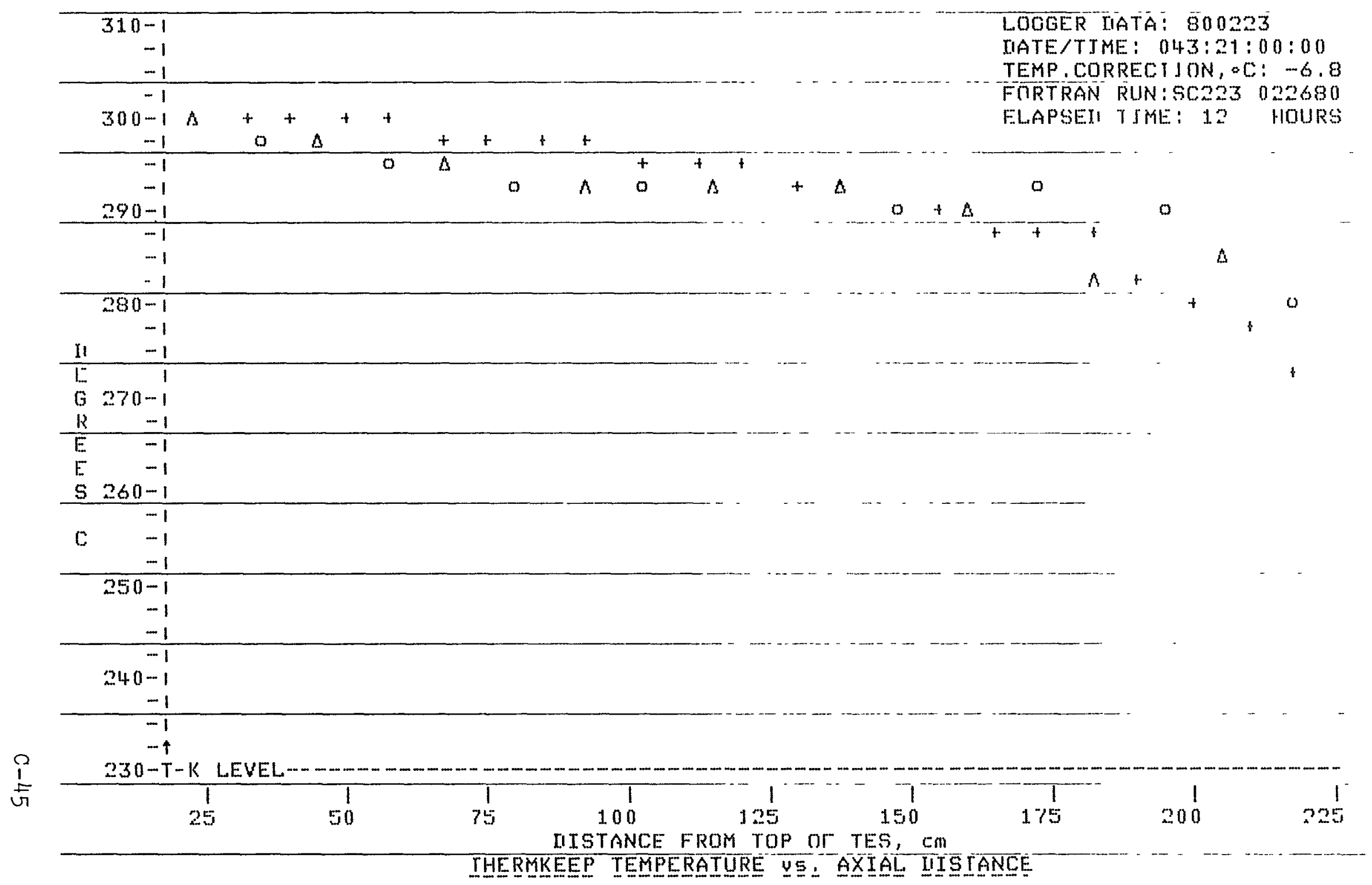

Figure C-43. Thermkeep temperature profile, solar cycle test 223 , at end of phase 2 discharge, Cycle 1. 


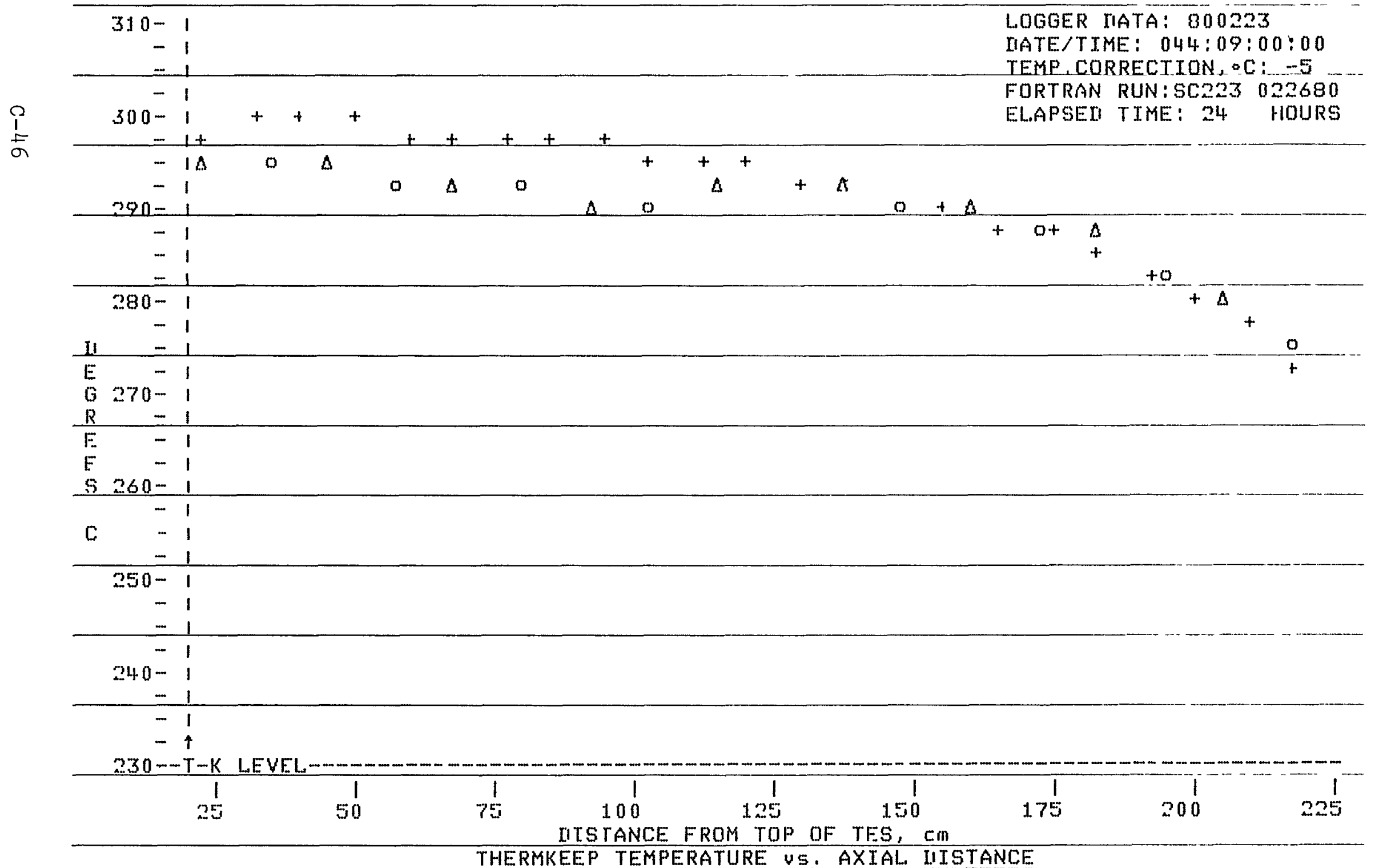

Figure C-44. Thermkeep temperature profile, solar cycle test 223, starting profile, cycle 2. 


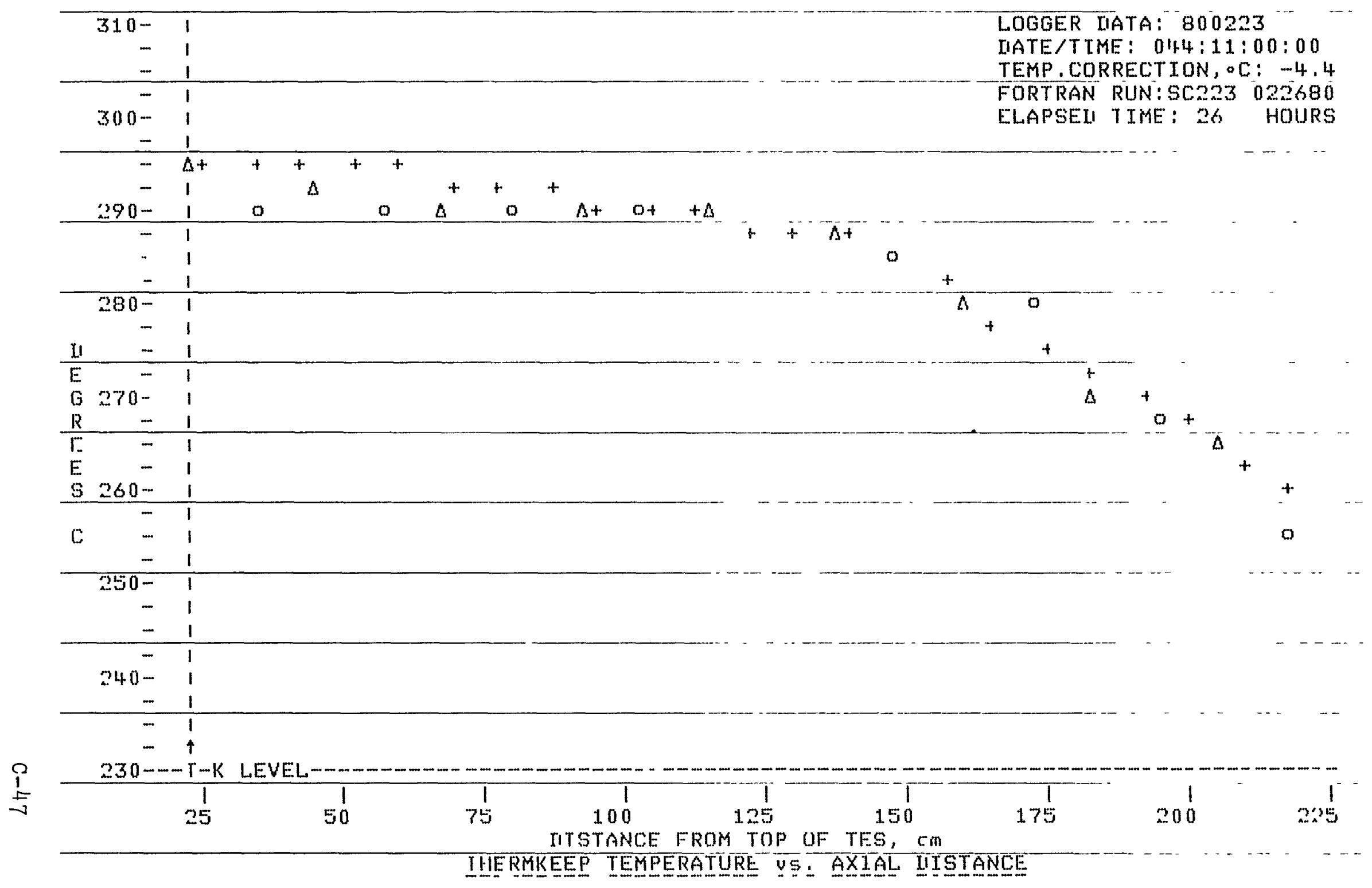

Figure C-45. Thermkeep temperature profile, solar cycle test 223, at end of phase 1 discharge, cycle 2. 


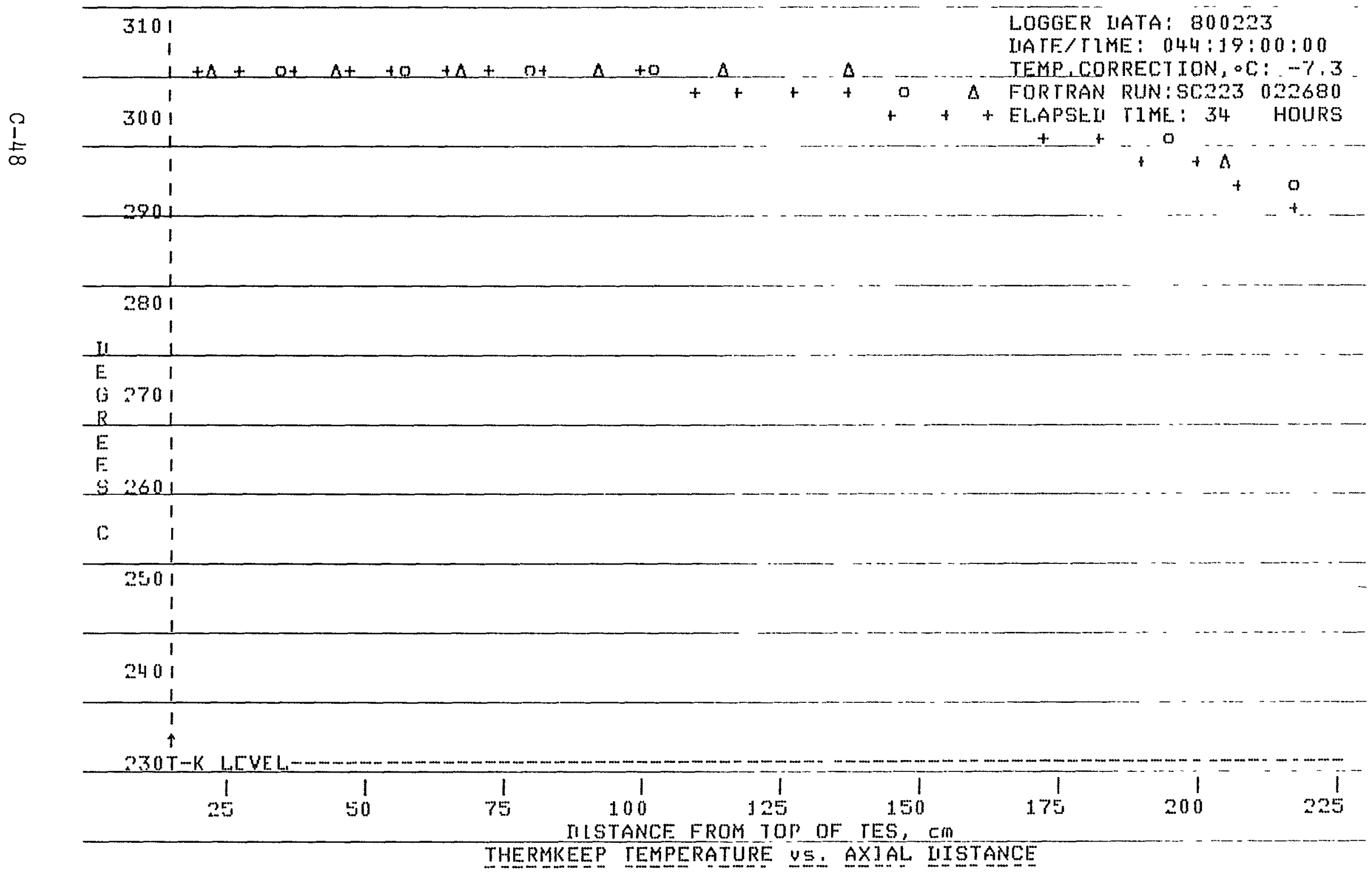

Figure C-46, Thermkeep temperature profile, solar cycle test 223, at end of cycle 2 charge, 


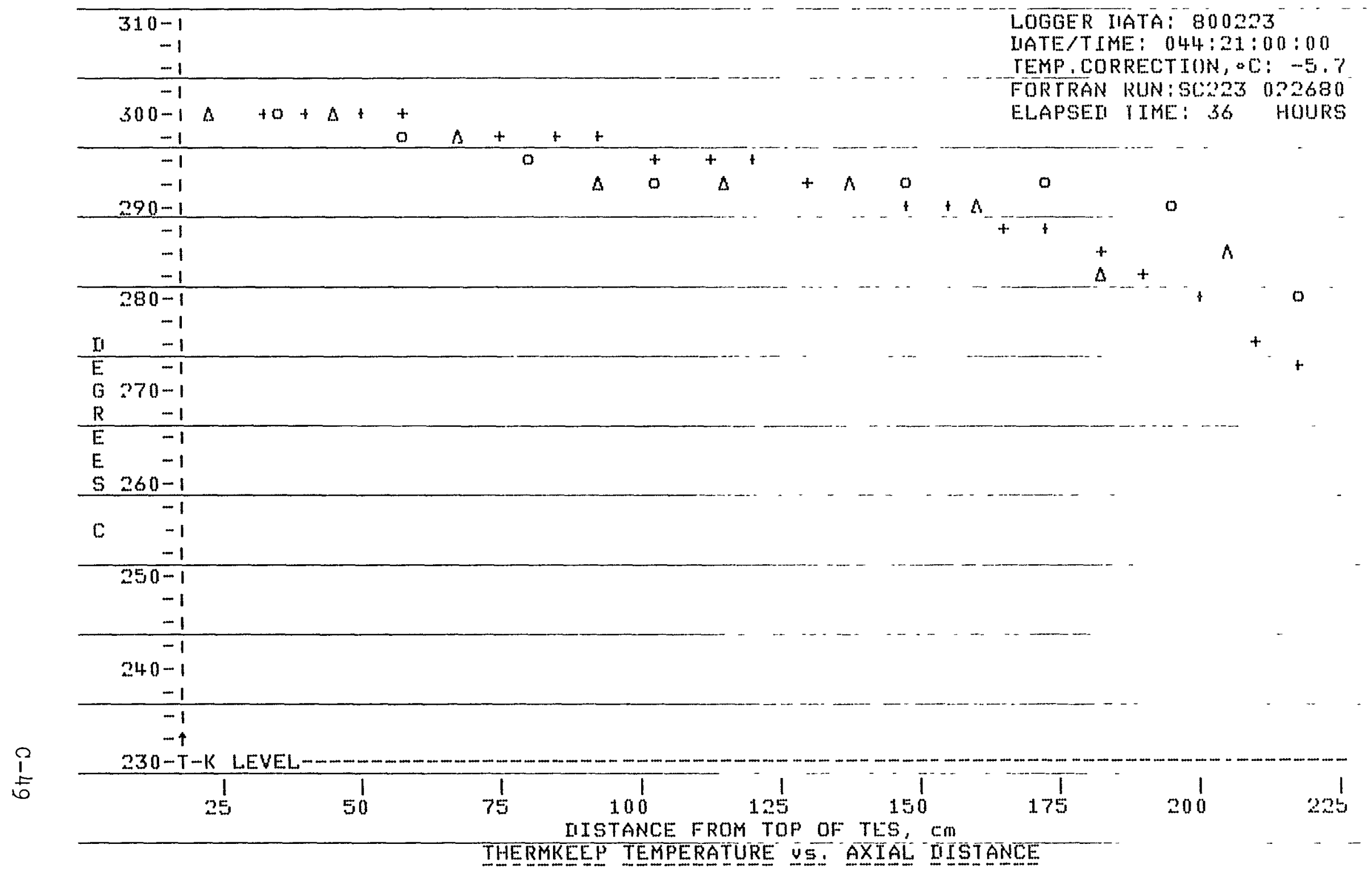

Figure C-47. Thermkeep temperature profile, solar cycle test 223 , at end of phase 2 discharge, cycle 2. 


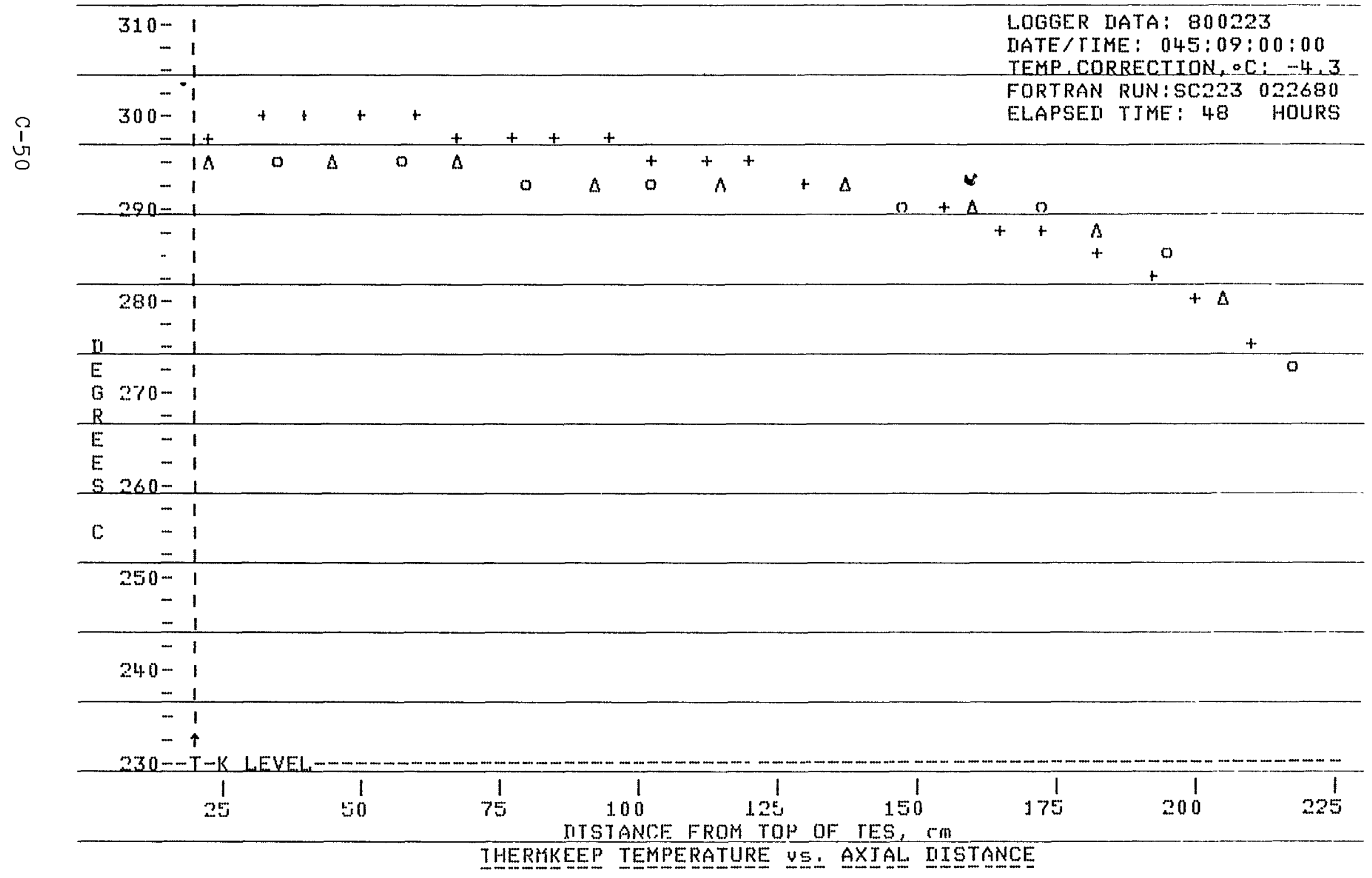

Figure C-48. Thermkeep temperature profile, solar cycle test 223 , at end of overnight idle after cycle 2. 


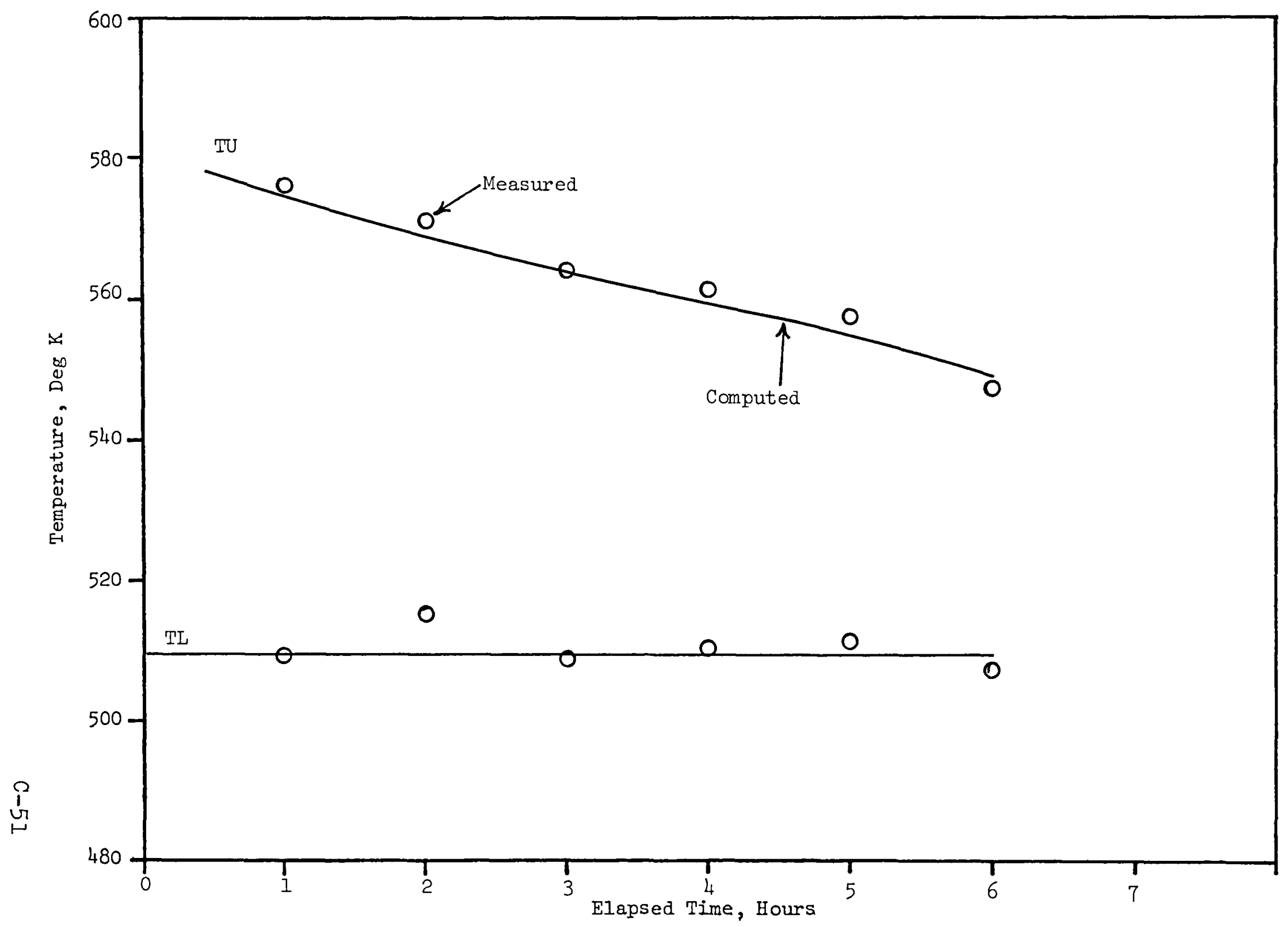

Figure C-49. T-66 outlet temperature, discharge test 001 (2.9 g gm) 


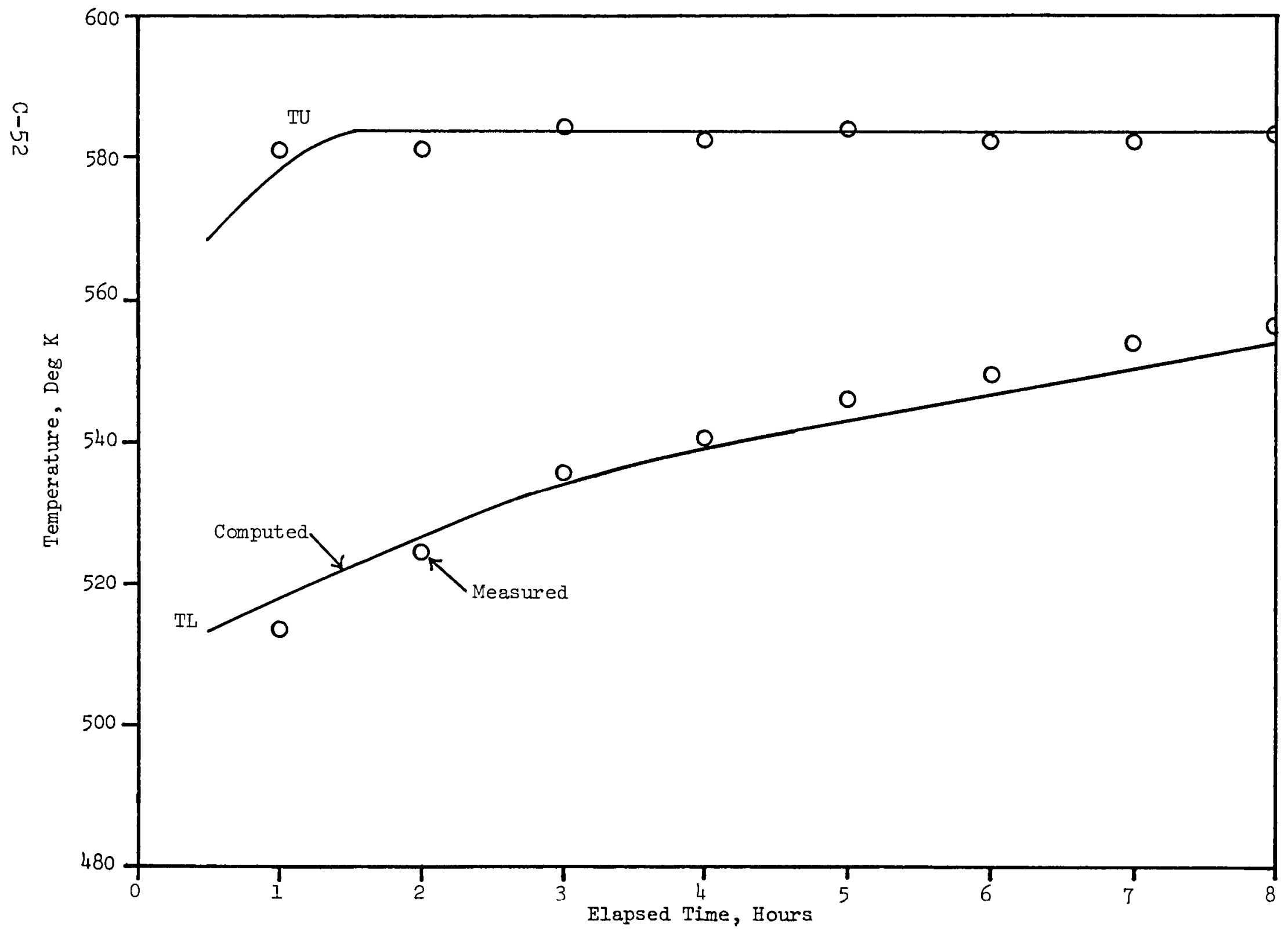

Figure C-50. T-66 outlet temperature, charge test $002(2.8 \mathrm{gpm})$. 


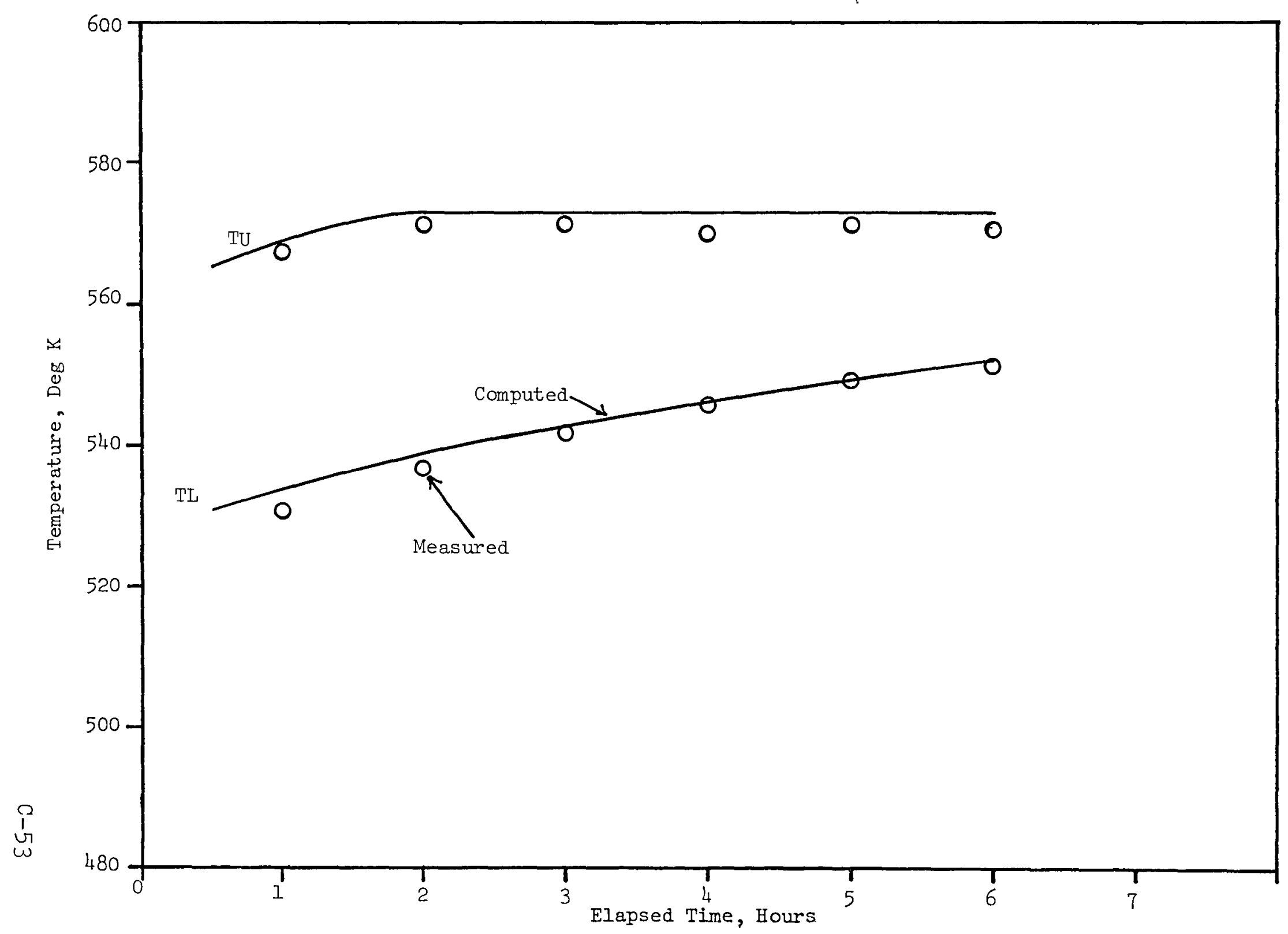

Figure C-51. T-66 outlet temperature, charge test 004 ( $3.4 \mathrm{gpm})$. 


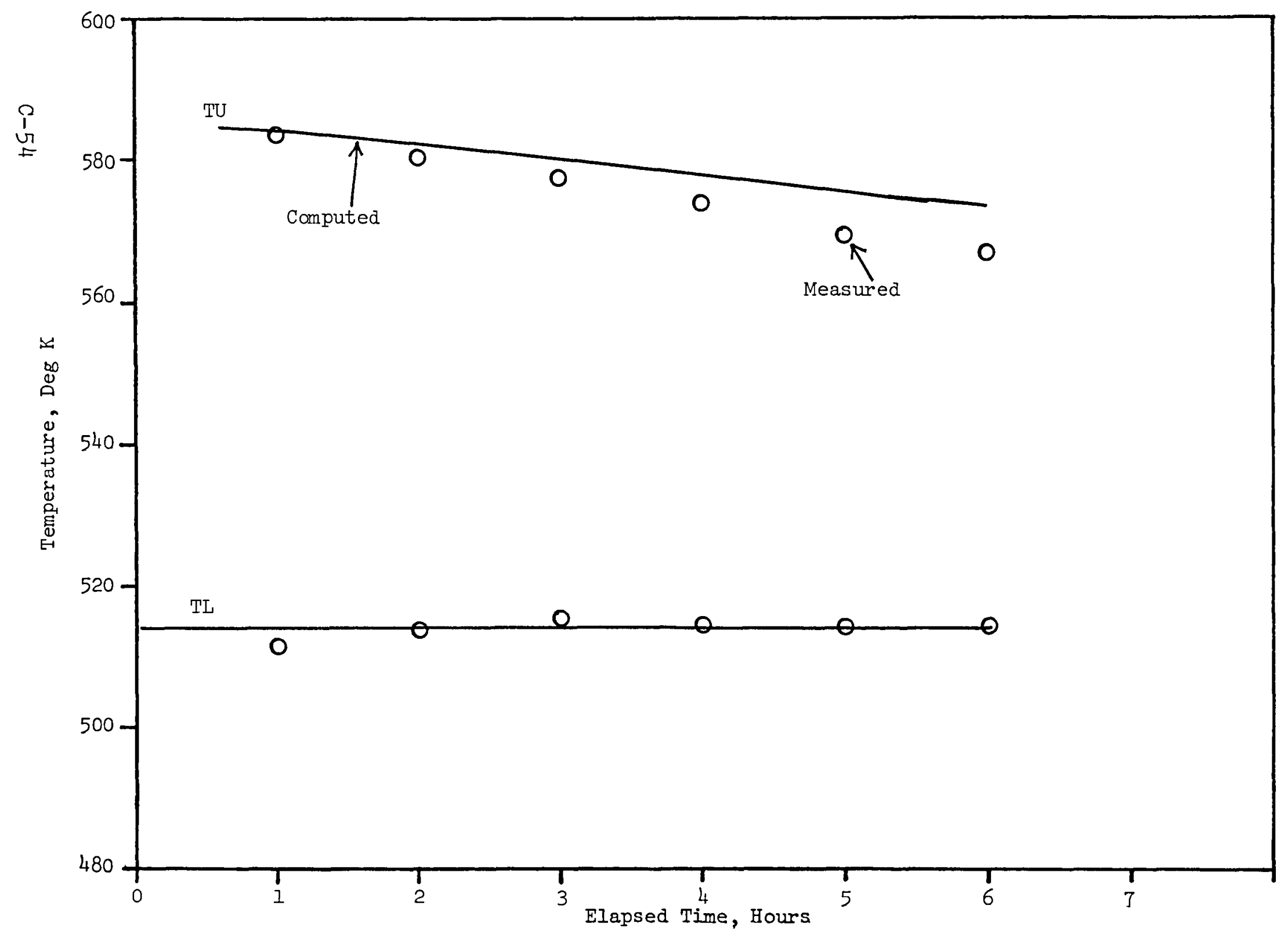

Figure C-52. T-66 outlet temperature, discharge test 014 ( $1,0 \mathrm{gpm}$ ). 


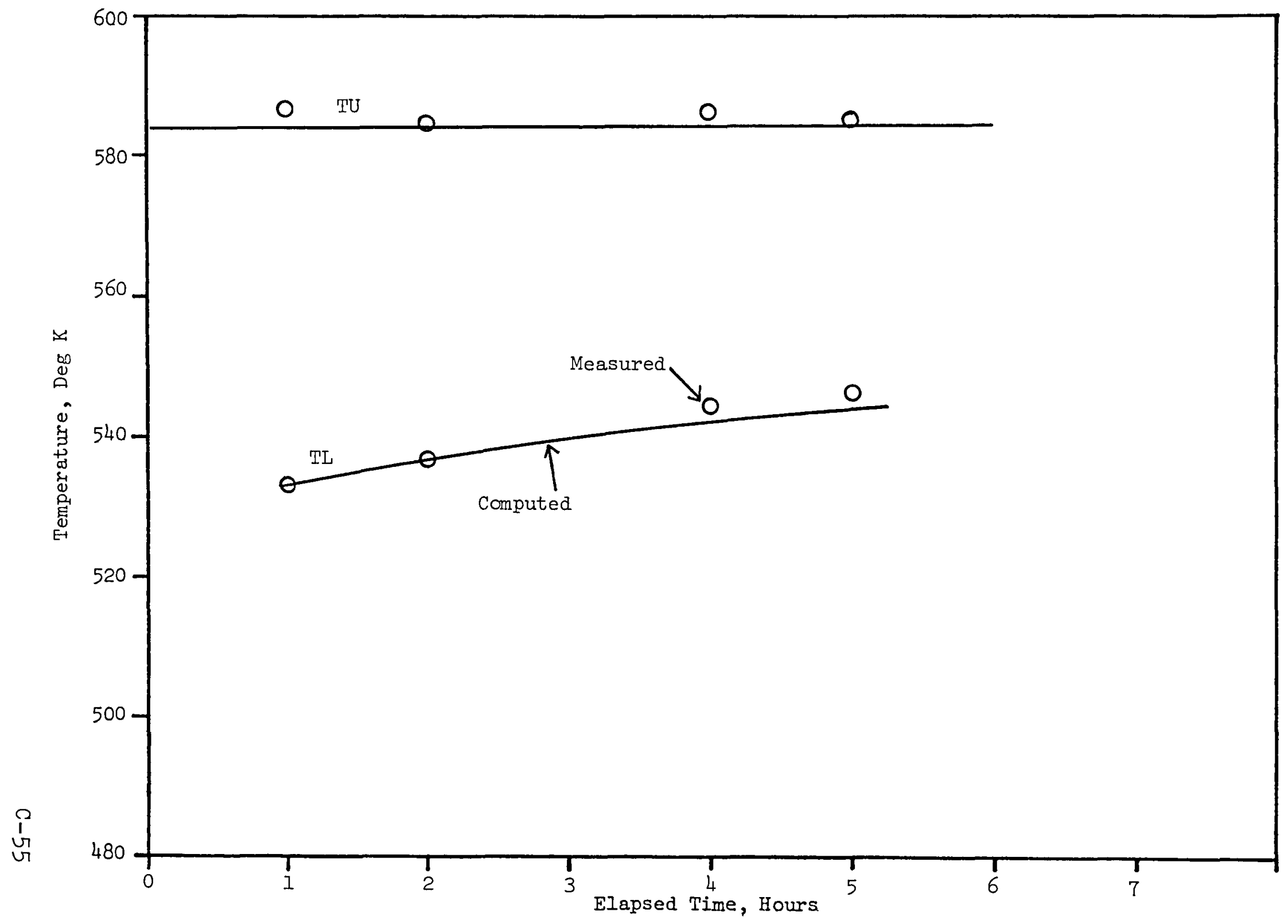

Figure C-53. T-66 outlet temperature, charge test 015 ( $1.0 \mathrm{gpm}$ ). 


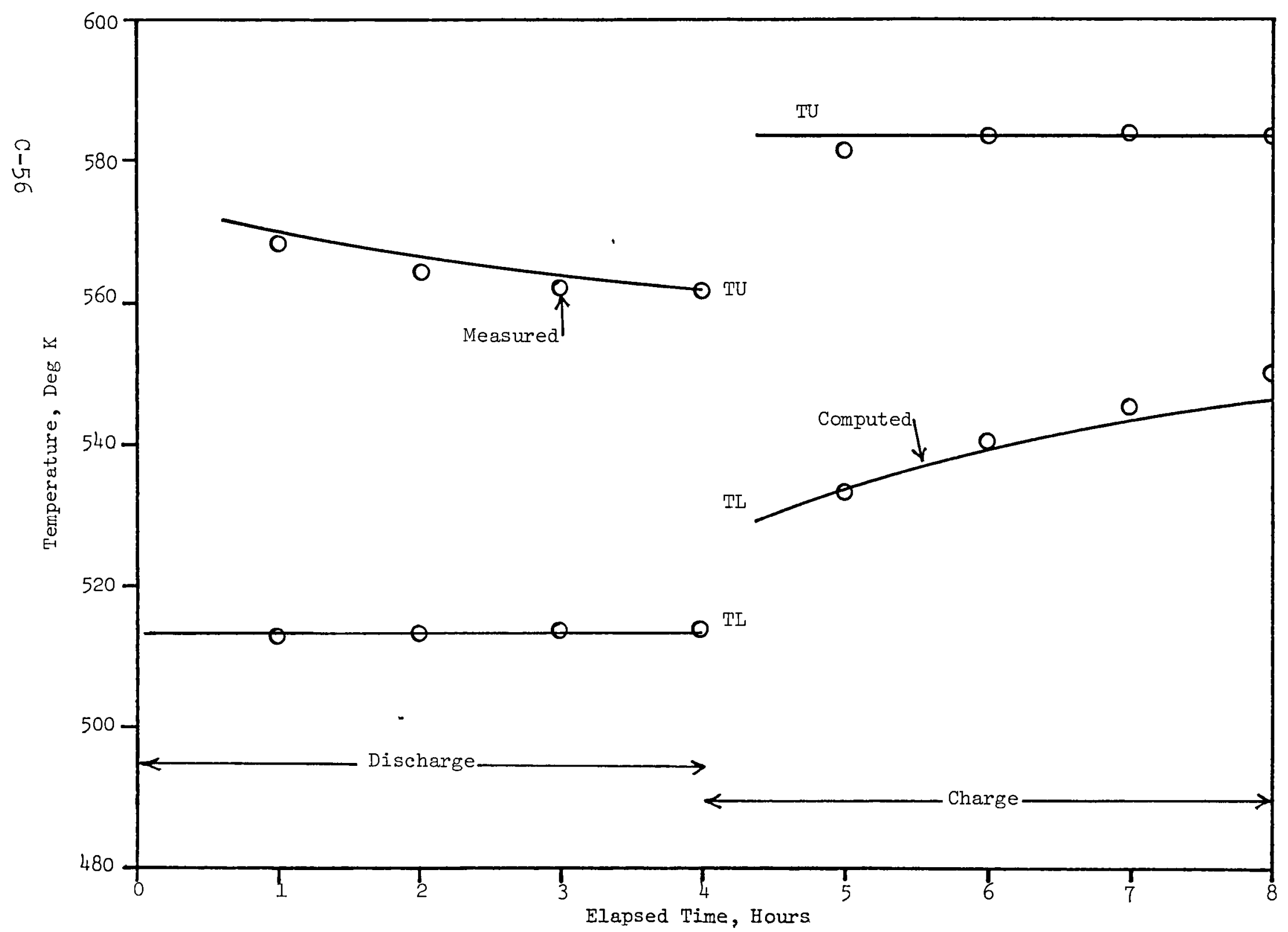

Figure C-54. T-66 outlet temperature, cyclic test 018 (2.0 g pm), first cycle. 


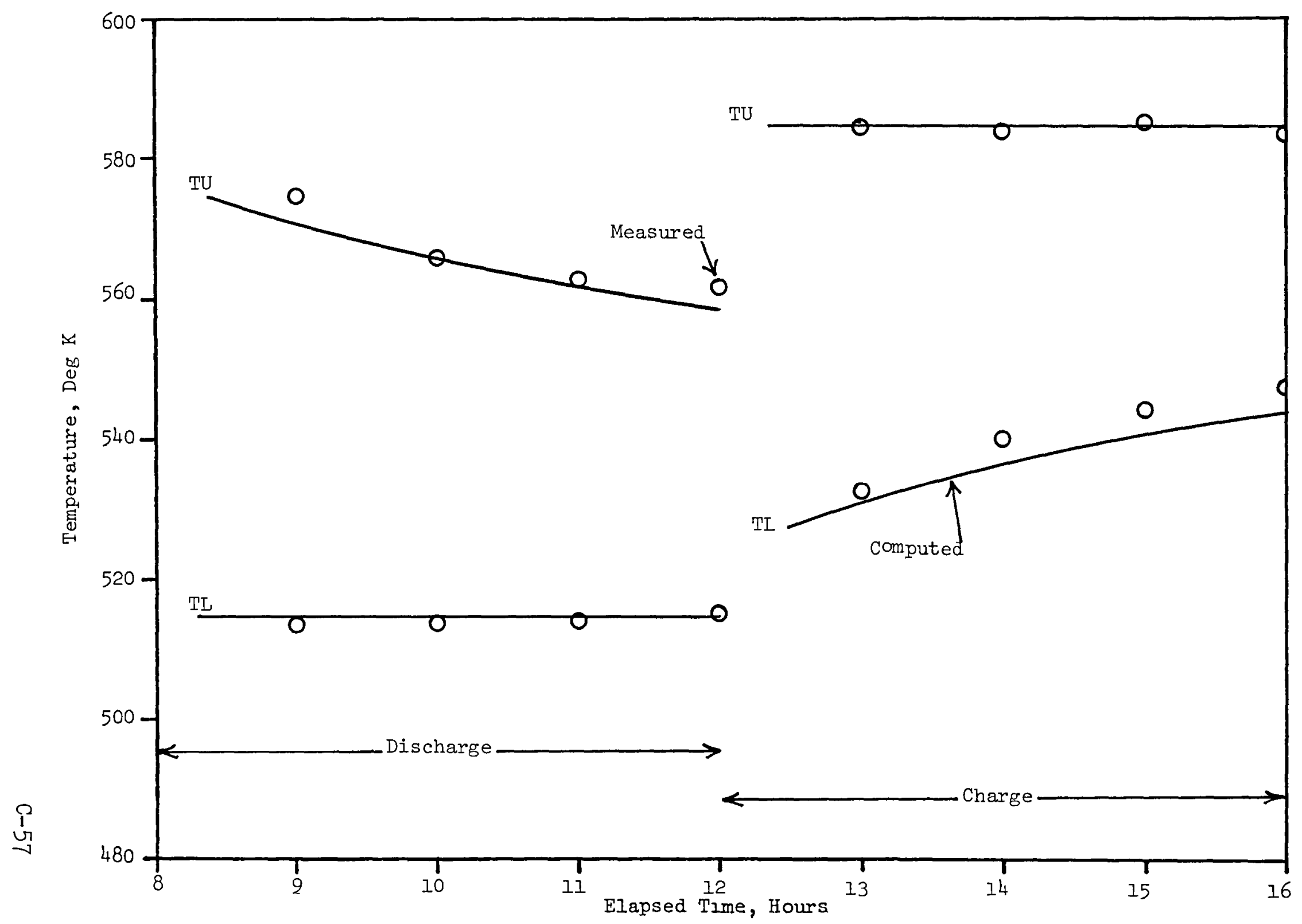

Figure C-55. T-66 outlet temperature, cycllc test 018 (2.0 gpm), second cycle. 


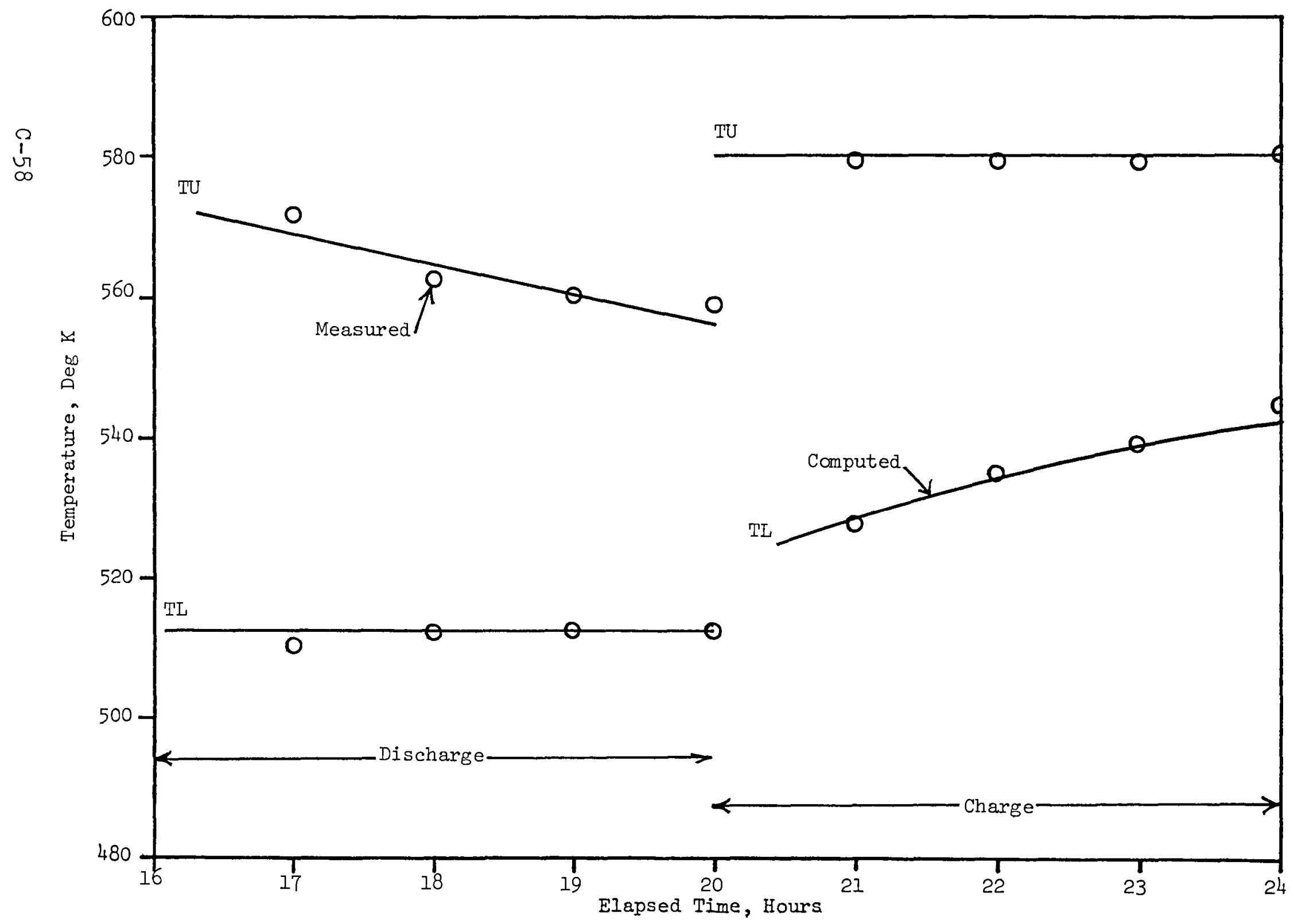

Figure C-56. T-66 outlet temperature, cyclic test 018 (2.0 gpm), third cycle. 


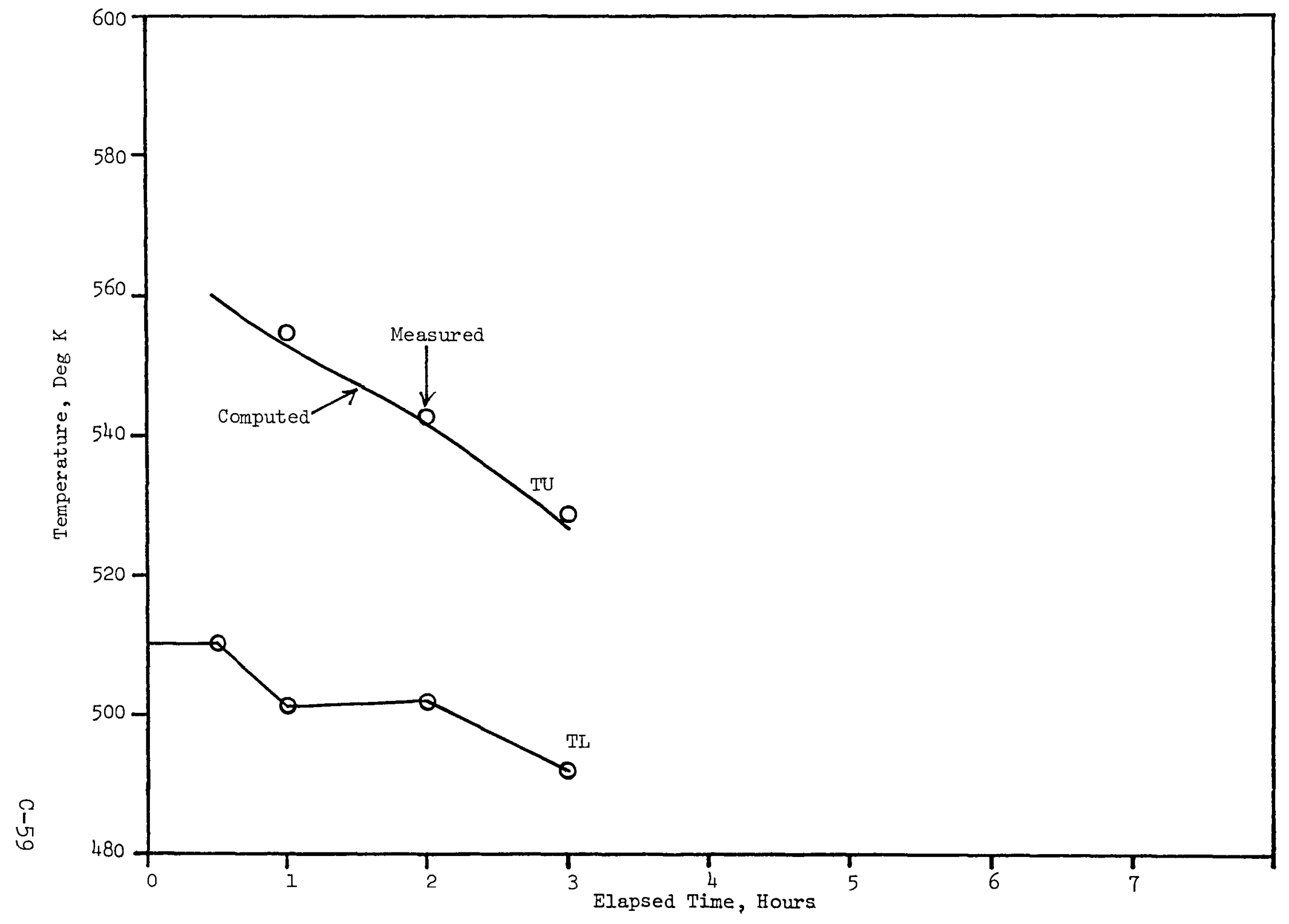

Figure C-57. T-66 outlet temperature, discharge test 019 (6.6. gpm) 


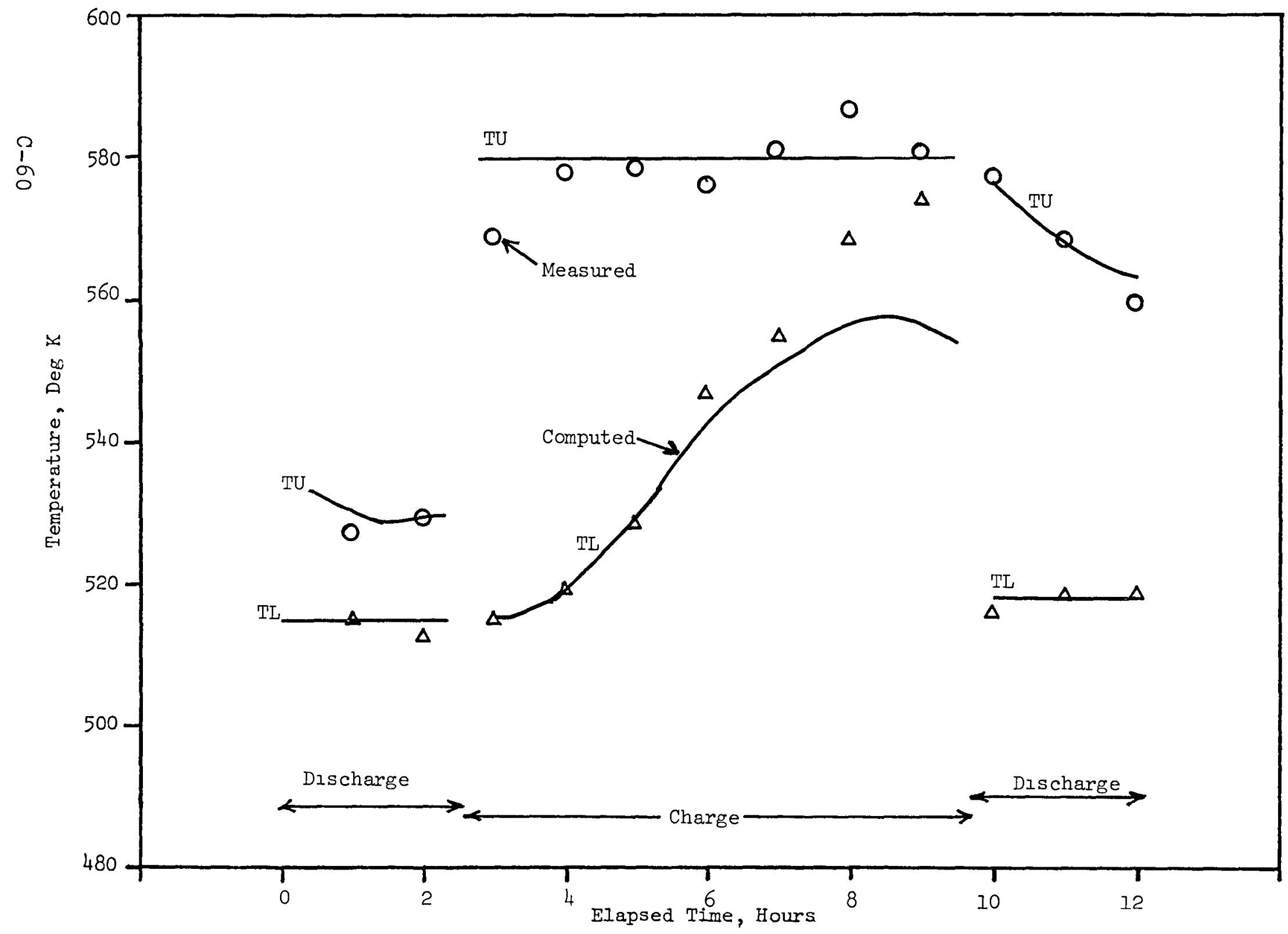

Figure C-58. T-66 outlet temperature solar cycle test 023 , first cycle. 


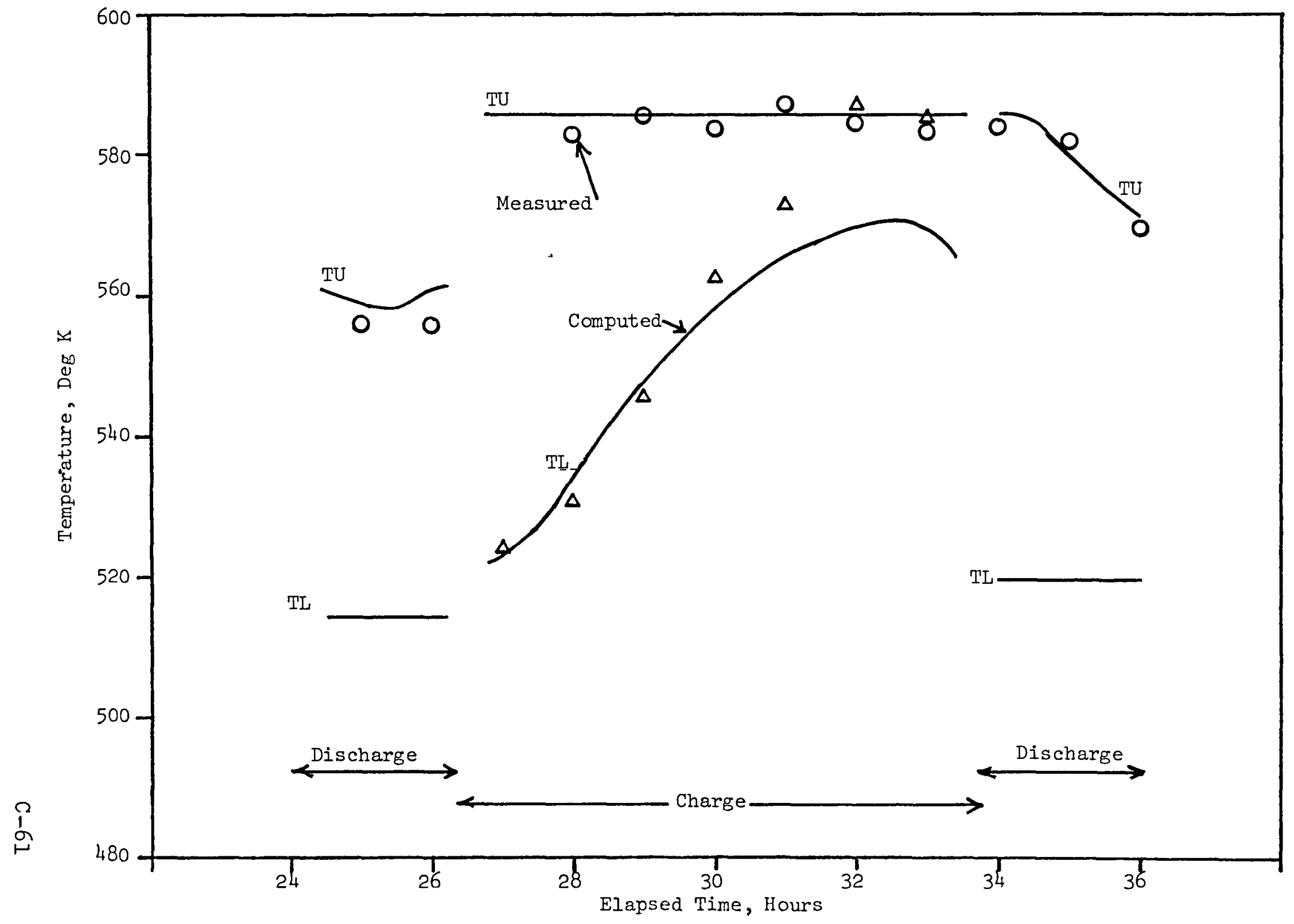

Figure C-59. T-66 outlet temperature solar cycle test 023 , second cycle. 


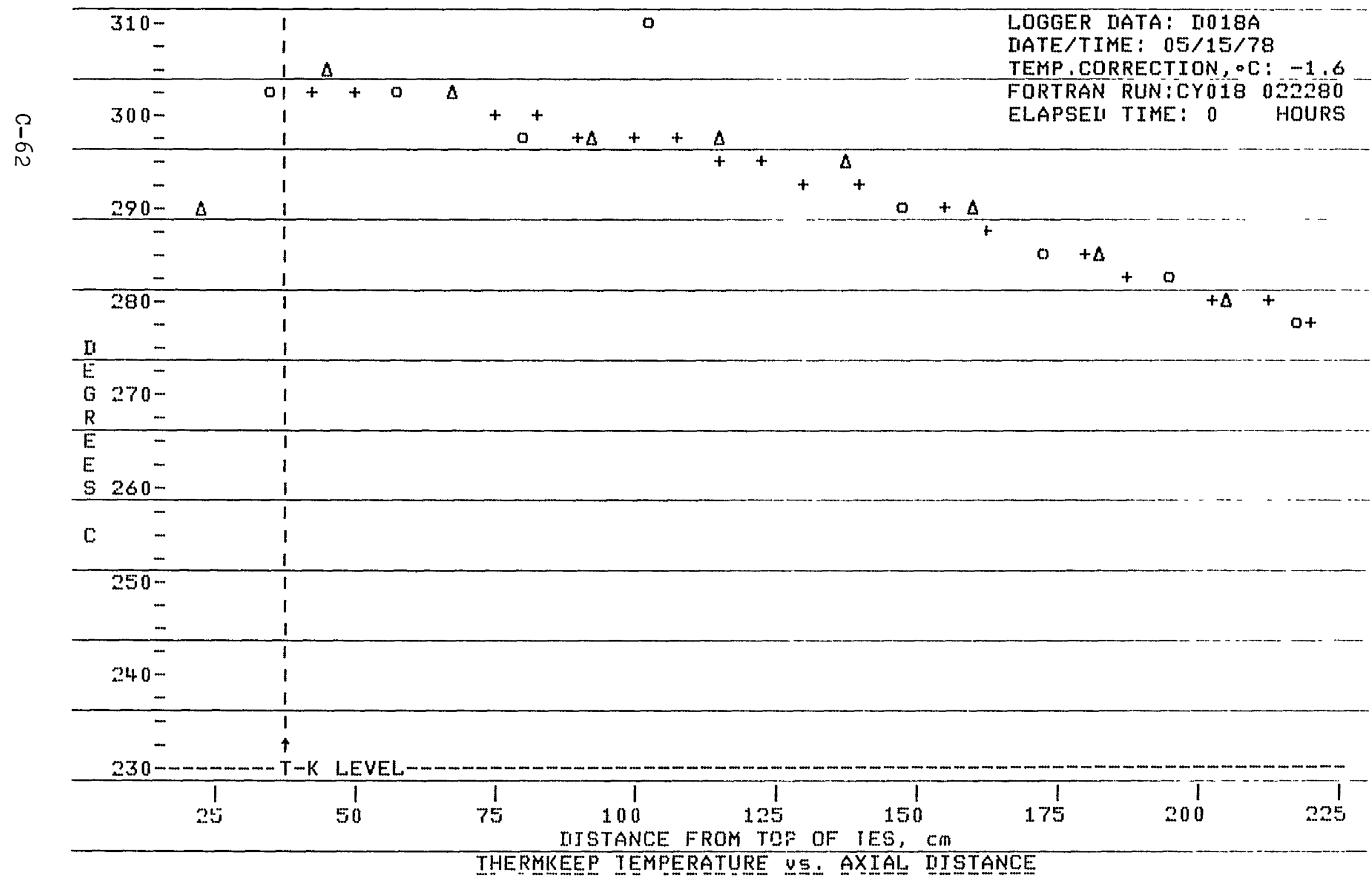

Figure C-60. Thermkeep temperature profile, cyclic test 018 (2.0 g pm), starting profile. 


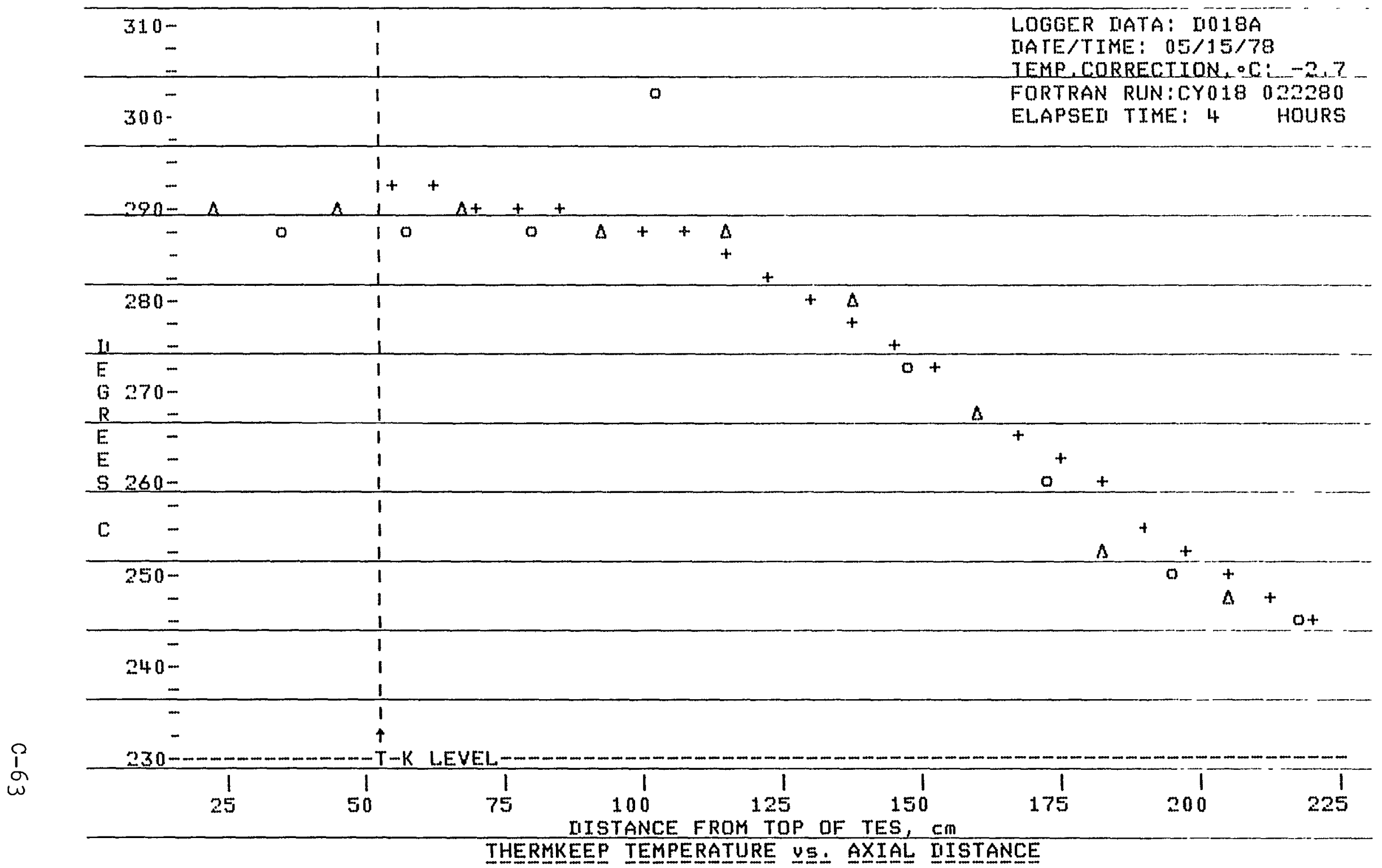

Figure C-61. Thermkeep temperature profile, cyclic test 018 (2.0 gpm), at end of first discharge phase. 


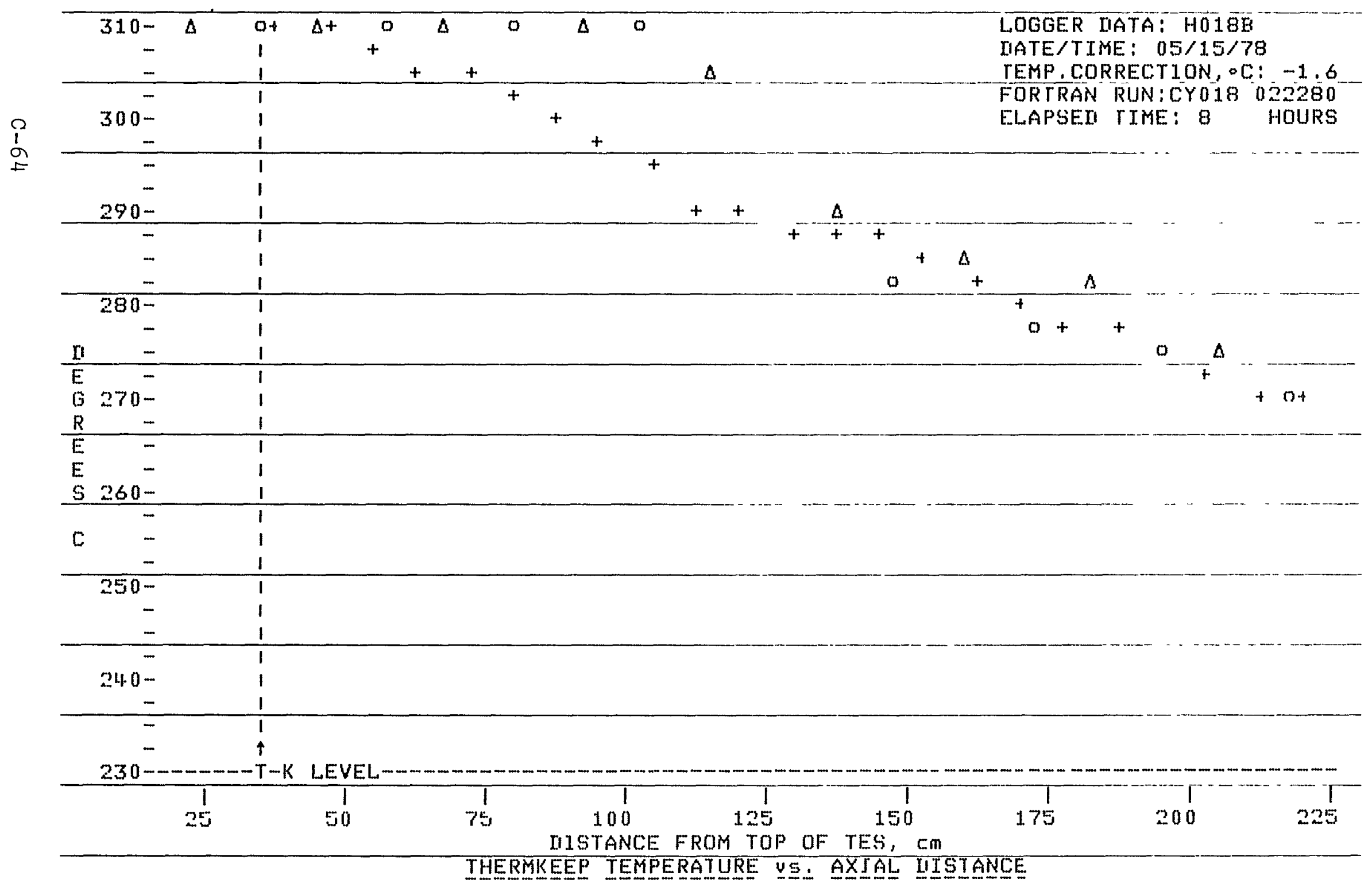

Figure C-62, Thermkeep temperature profile, cyclic test 018 (2.0 gom), at end of first charge phase. 


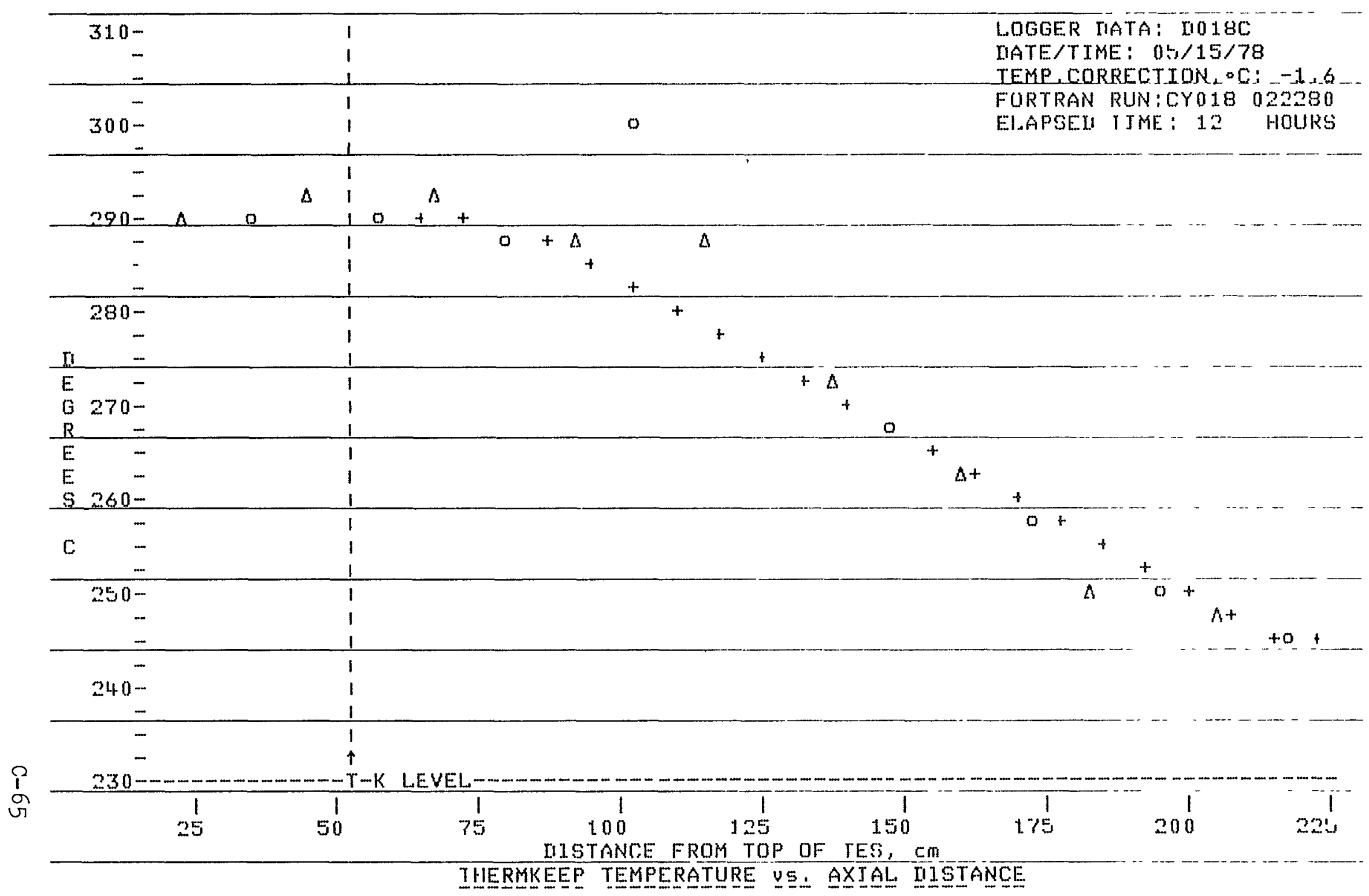

Figure C-63. Thermkeep temperature proflle, cyclic test 018 (2.0 gpm), at end of second discharge phase. 


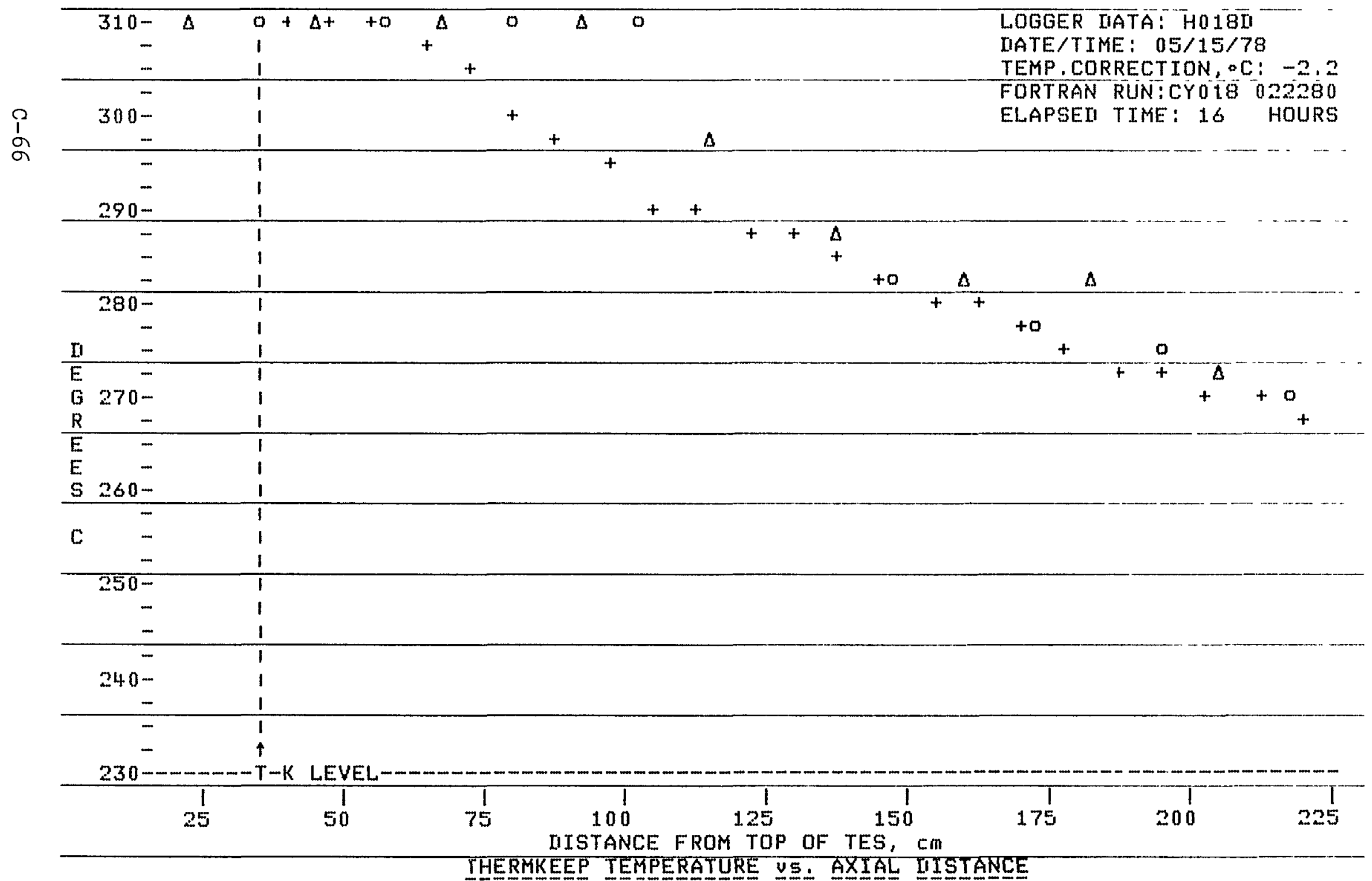

Figure C-64. Thermkeep temperature profile, cyclic test $018(2,0 \mathrm{gpm})$, at end of second charge phase. 


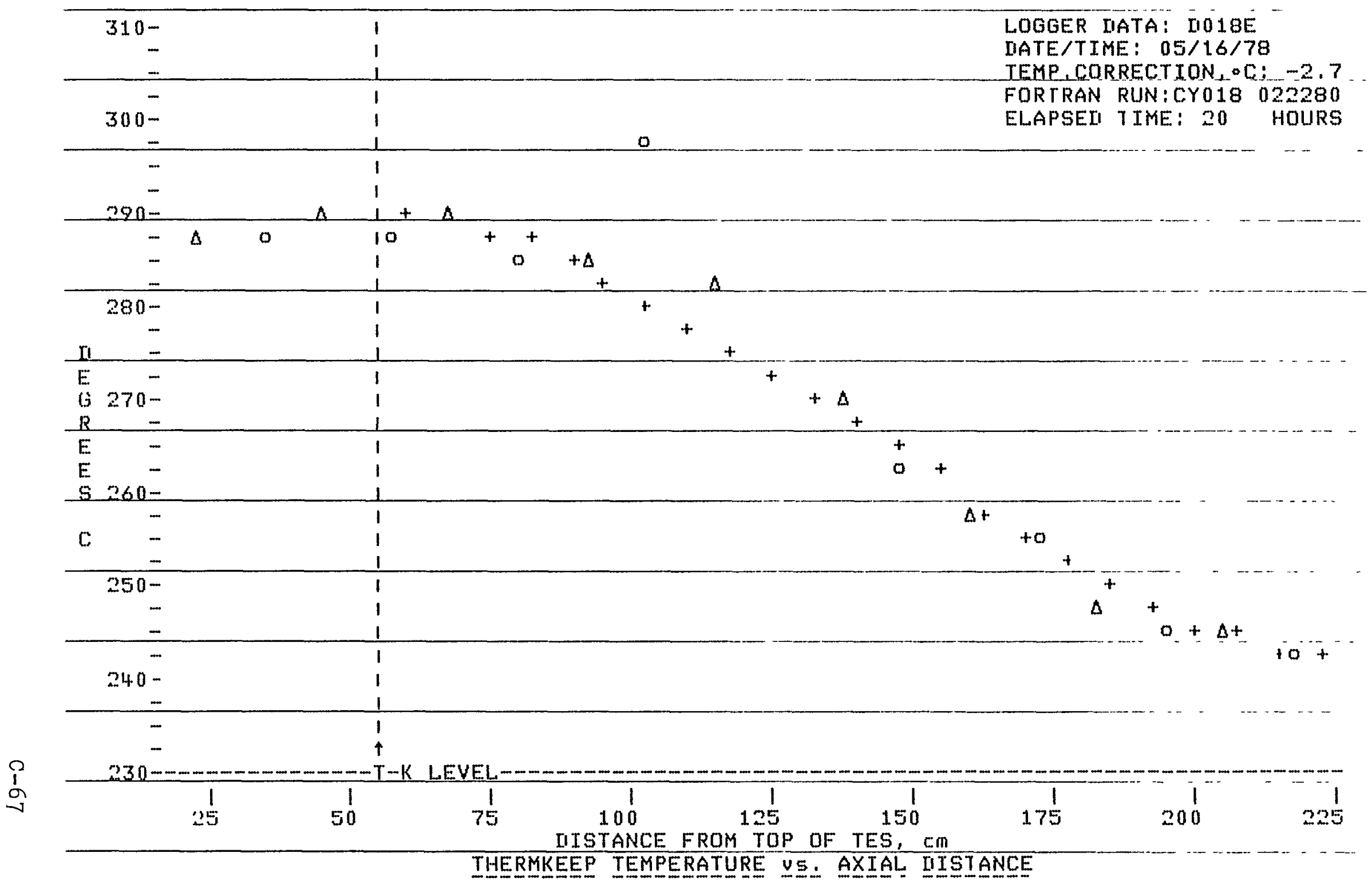

Figure C-65. Thermkeep temperature profile, cyclic test $018(2.0) \mathrm{gpm}$ ), at end of third discharge phase. 


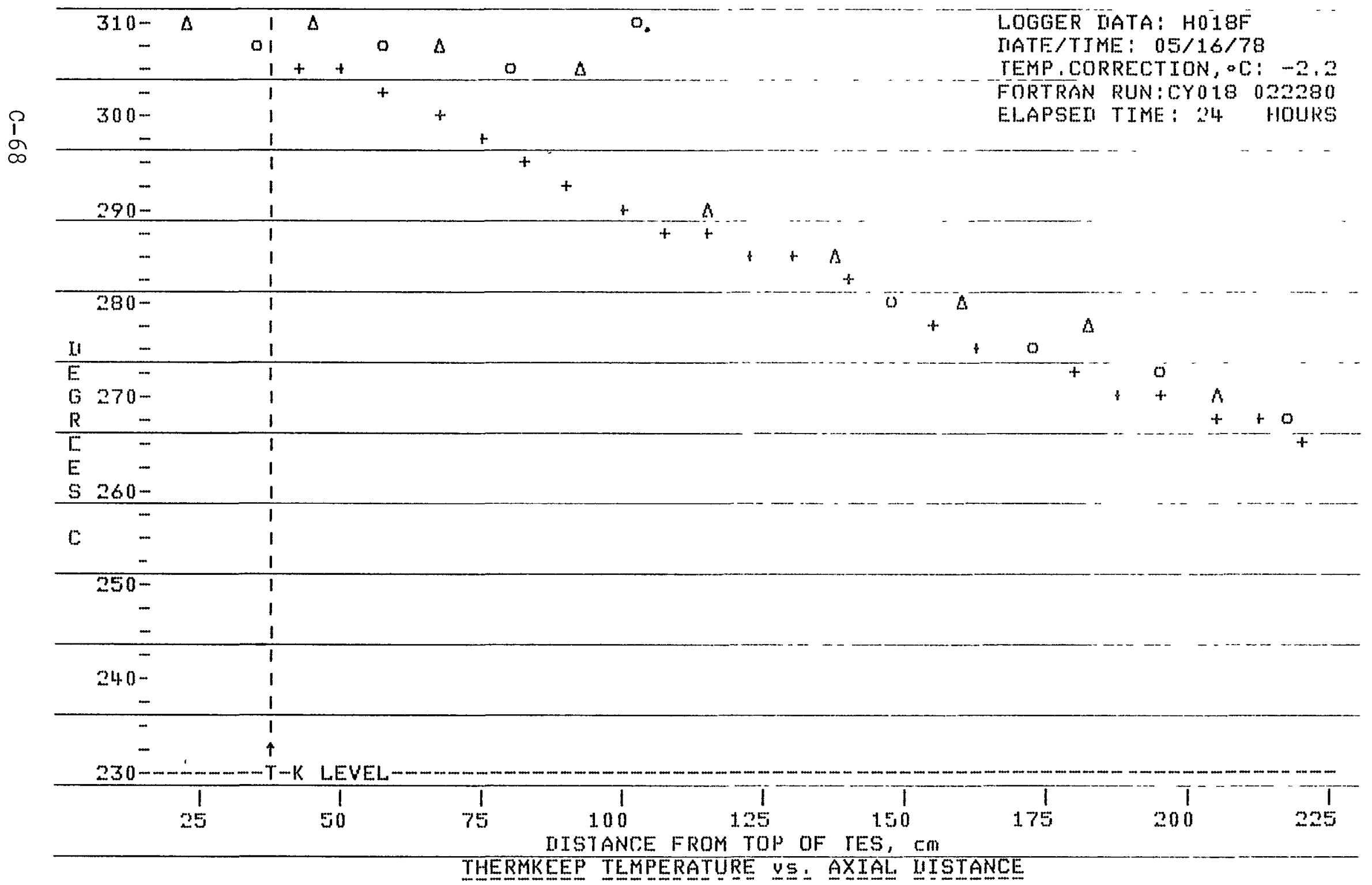

Figure C-66. Thermkeep temperature profile, cyclic test $018(2.0 \mathrm{gpm})$, at end of third charge phase. 


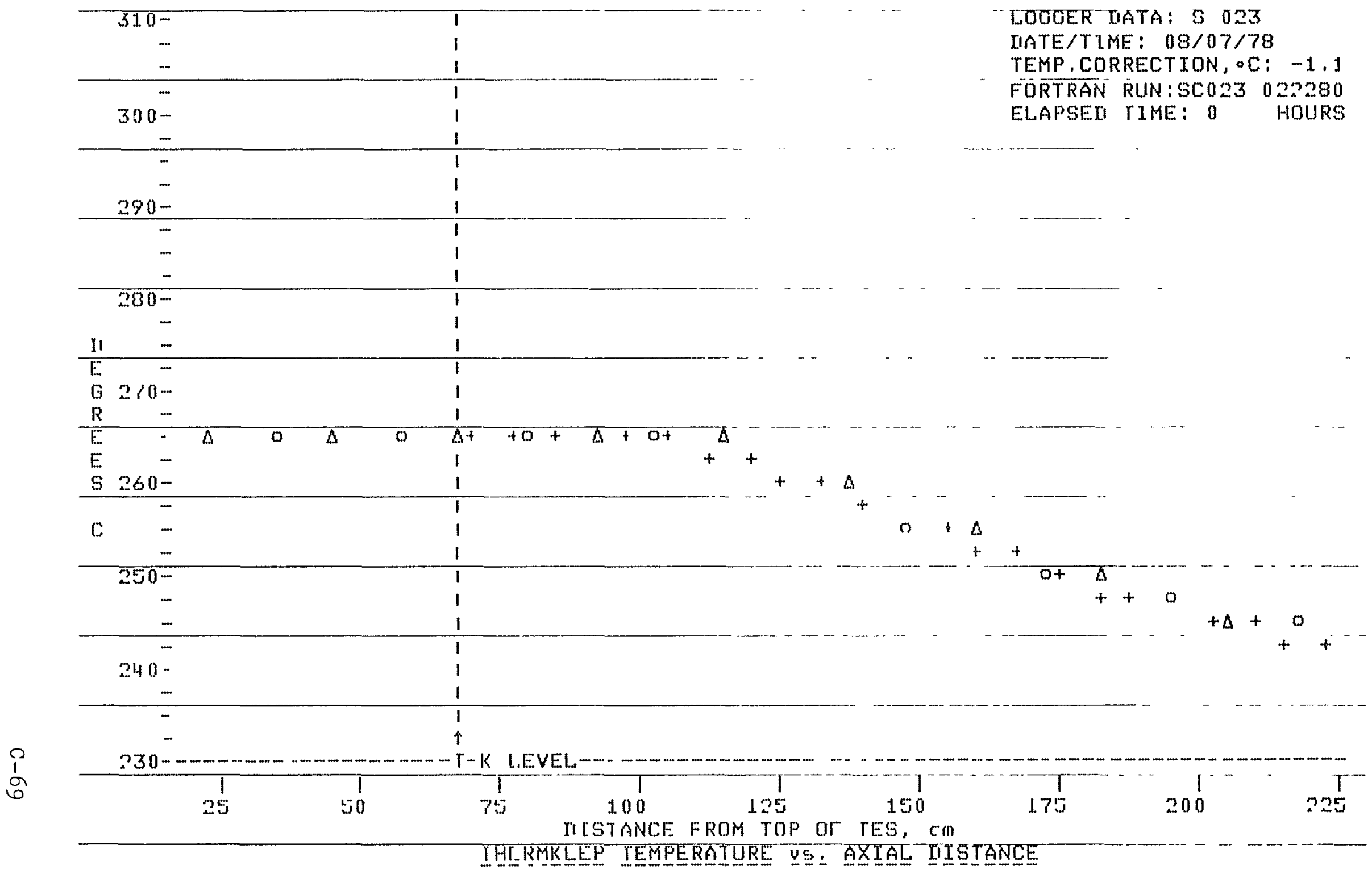

Figure C-67. Thermkeep temperature profile, solar cycle test 023, starting proflle, cycle 1. 


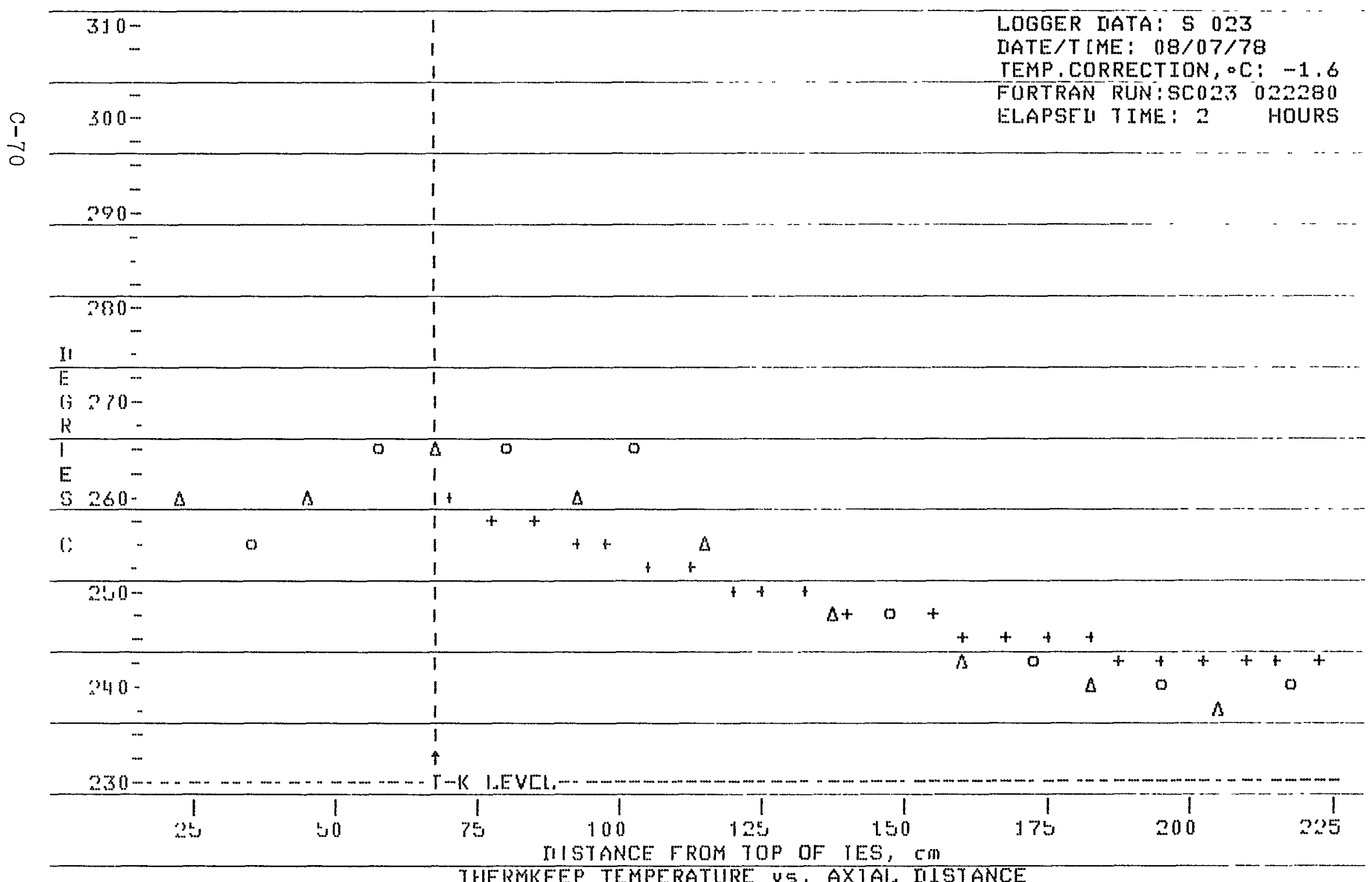

Fıgure C-68. Thermkeep temperature profile, solar cycle test 023, at end of phase 1 discharge, cycle 1 . 


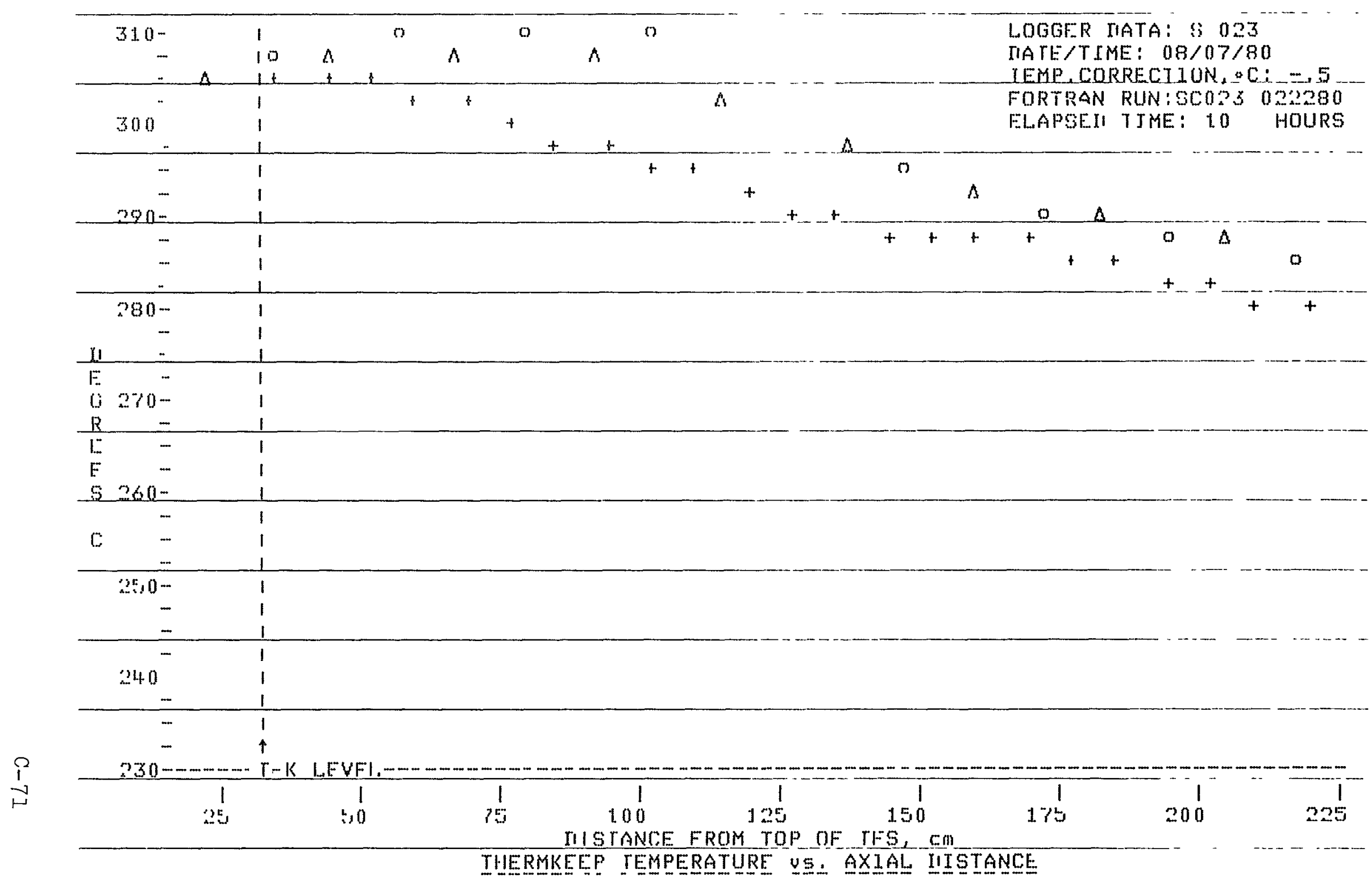

Figure C-69. Thermkeep temperature profile, solar cycle test 023, at end of cycle 1 charge. 


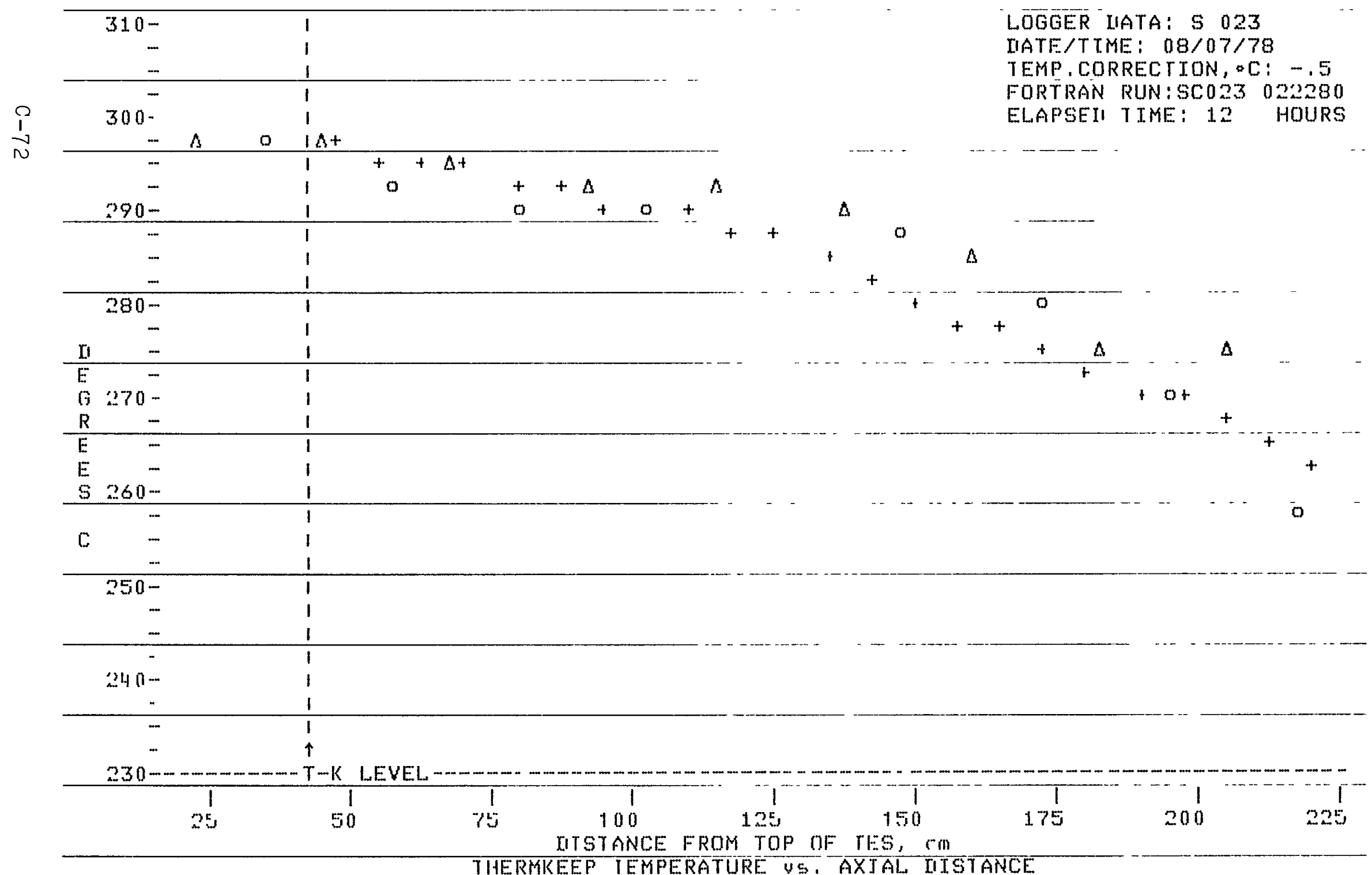

Figure C-70. Thermkeep temperature profile, solar cycle test 023 , at end of phase 2 discharge, cycle 1. 


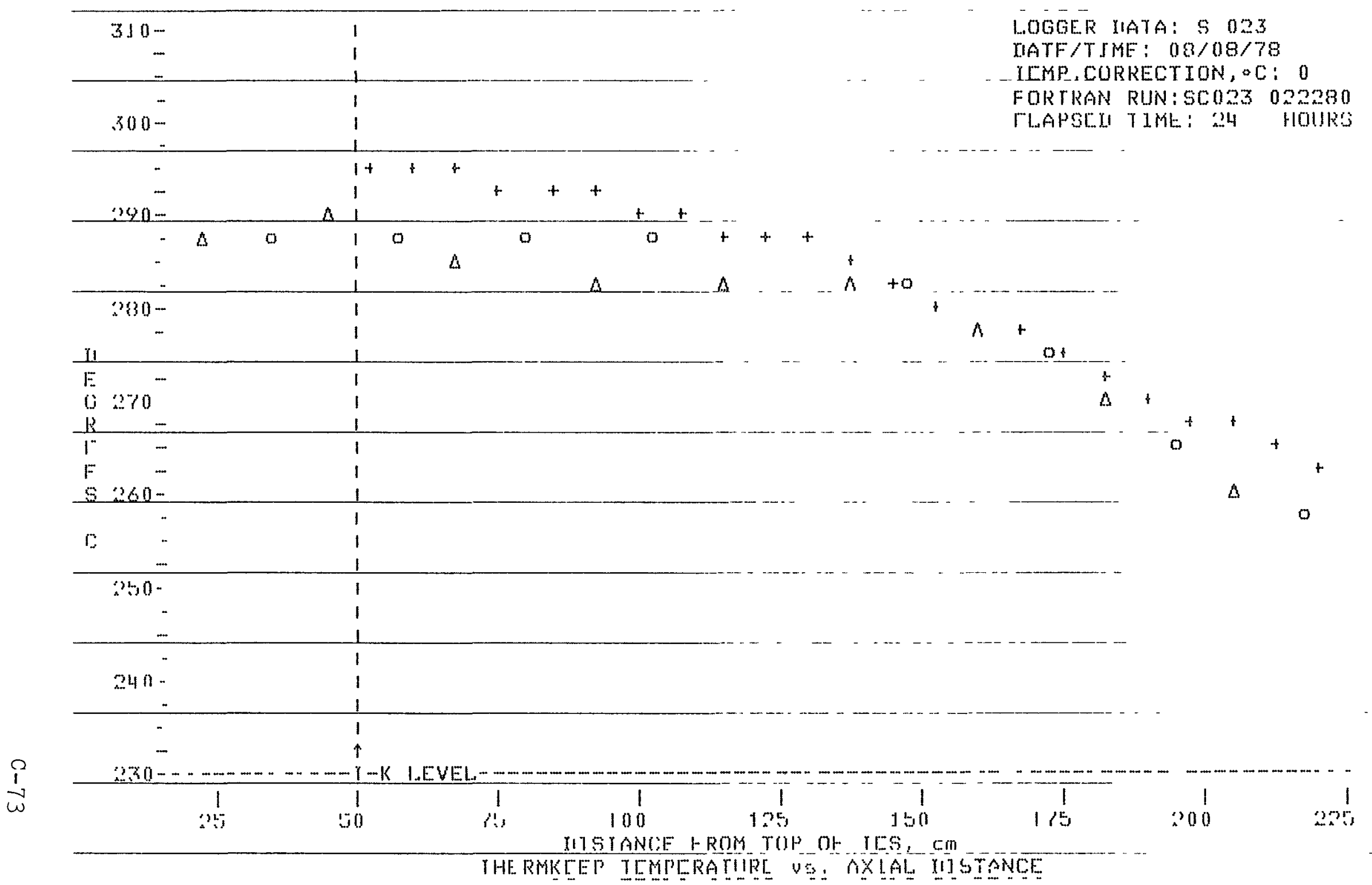

Figure C-7l. Thermkeep temperature profile, solar cycle test 023, starting profile, cycle 2. 


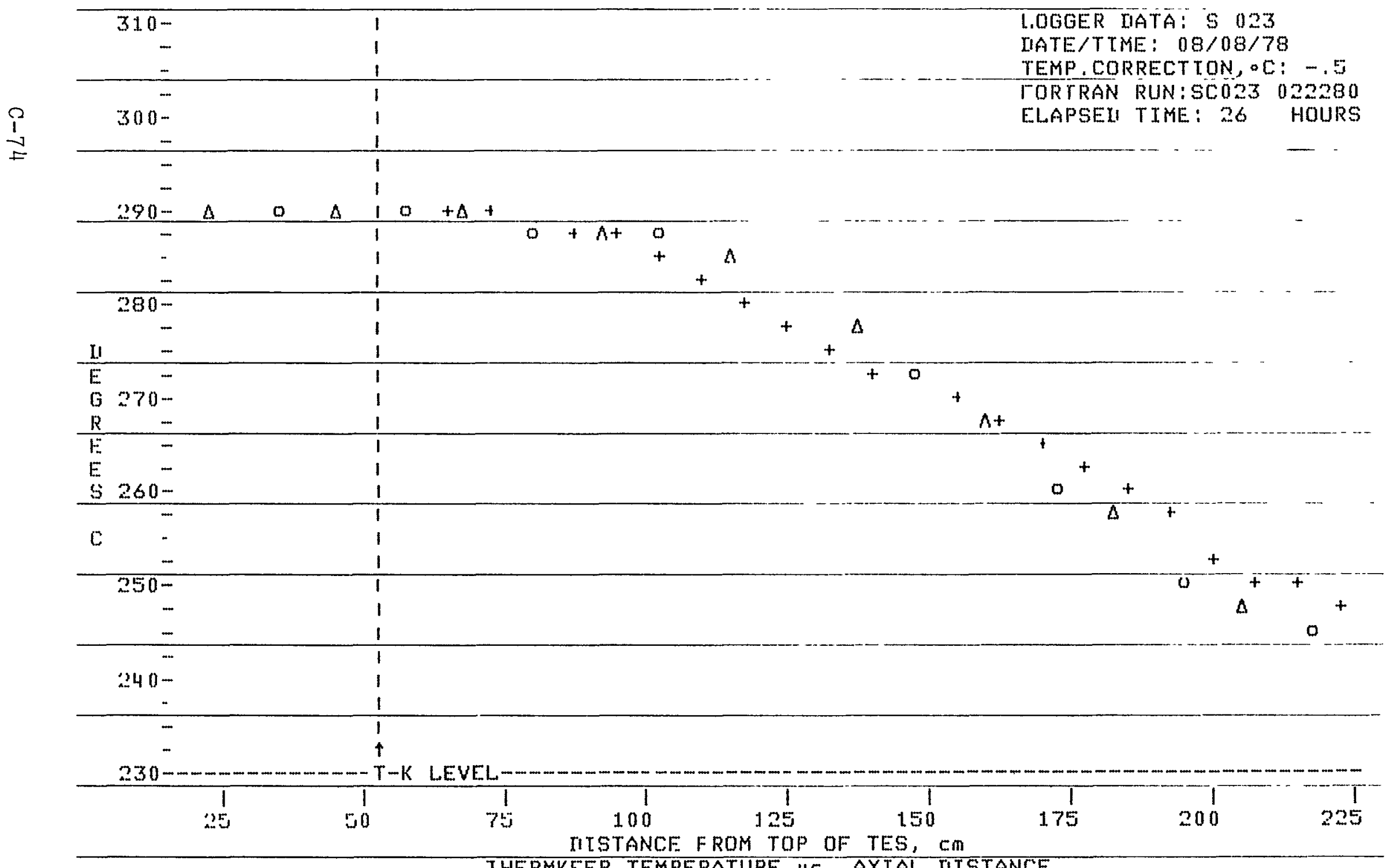

IHERMKEEP TEMPERATURE QS. AXIAL IIISTANCE

Figure C-72. Thermkeep temperature profile, solar cycle test 023 , at end of phase 1 discharge, cycle 2. 


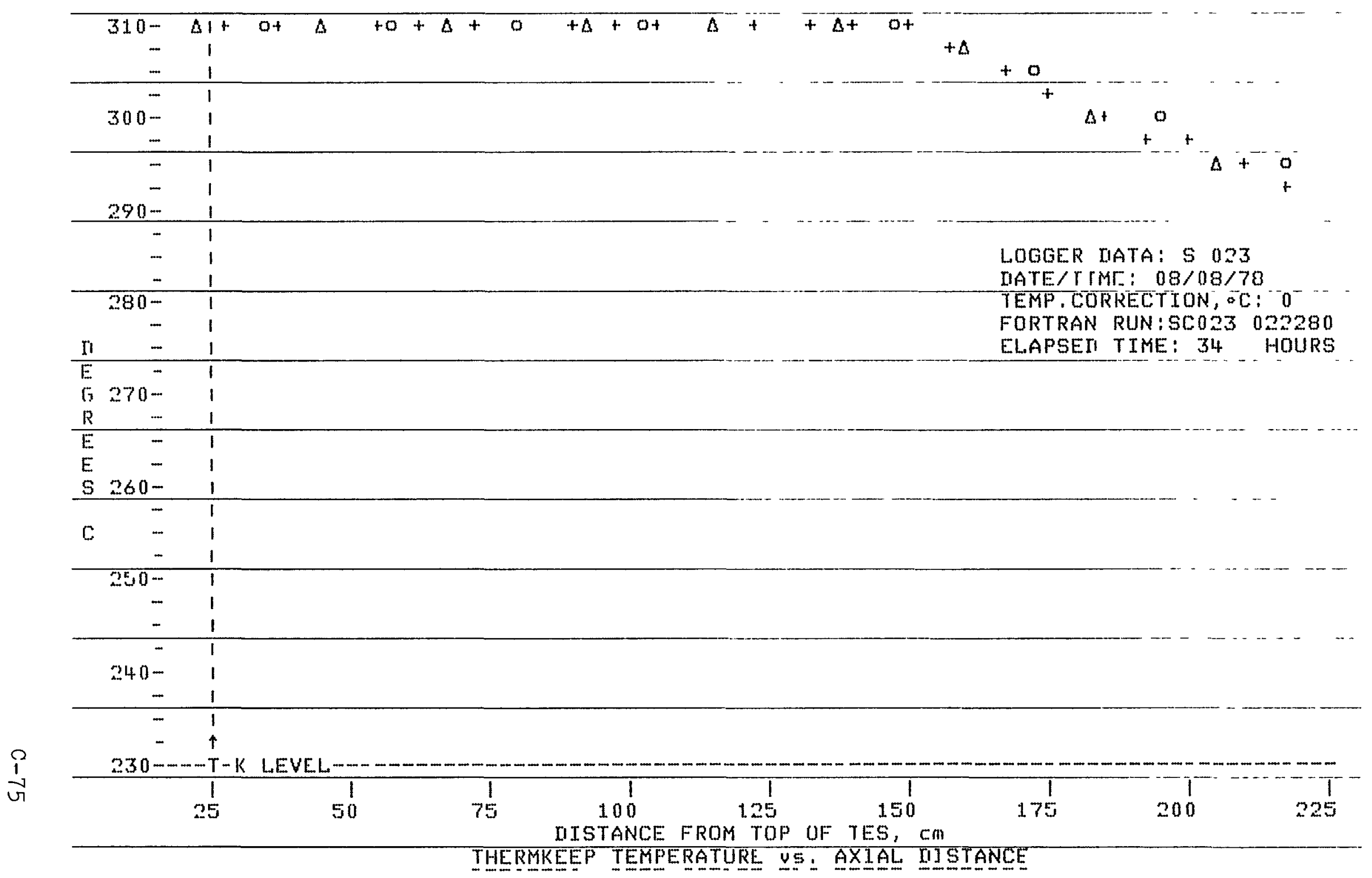

Figure C-73. Thermkeep temperature profile, solar cycle test 023, at end of cycle 2 charge. 


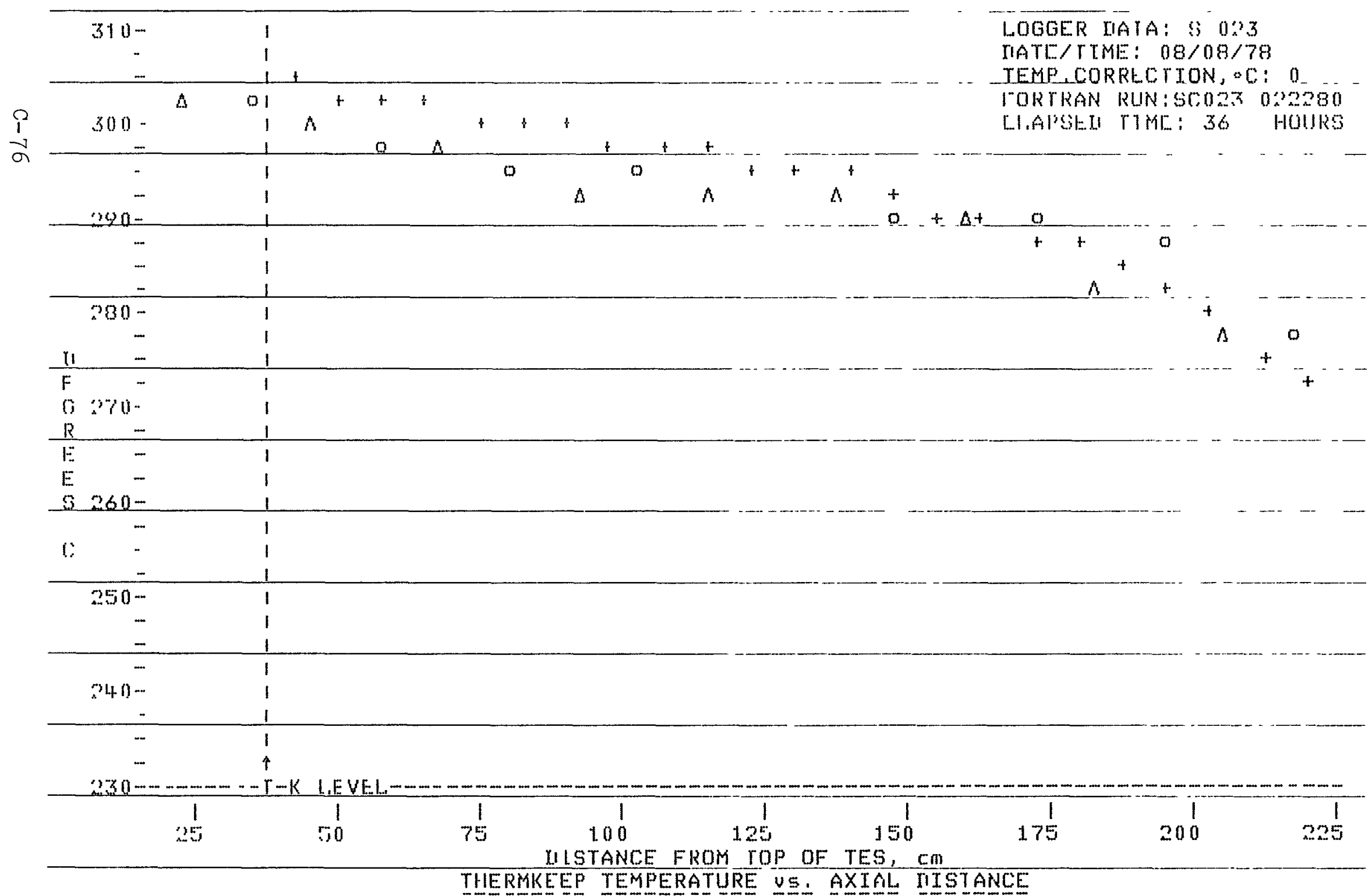

Flgure C-74. Thermkeep temperature proflle, solar cycle test 023, at end of phase 2 dlscharge, cycle 2. 


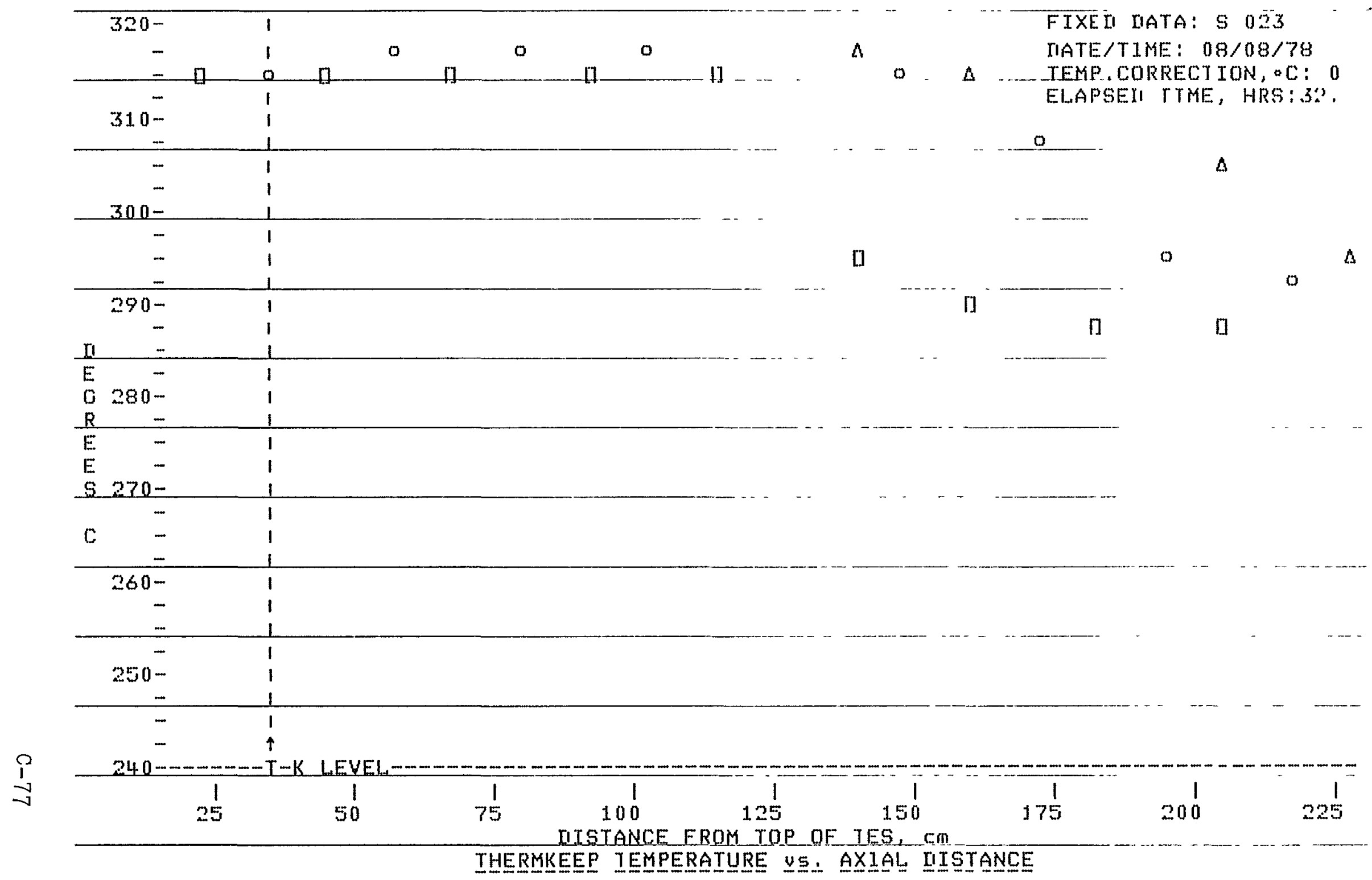

Figure C-75. Thermkeep temperature profile, solar cycle test 023 , at 32 hours. 



\author{
$\begin{array}{lllllllllllllll}\text { A } & P & P & E & N & D & I & X & D\end{array}$
}

USE OF COMPUTER PROGRAM

\title{
Input Data
}

In order to run a test case with the FORTRAN program, data must be entered into the DATA section in the proper format. Each piece of data which is read by the program is then identified and printed in the output section of every run to provide easy checking of all parameters. There are three main groups of data:

\section{Main Program Data}

A table of solar collector output vs. time, fluid characteristics, and program control data (Fig. D-l). These are self-explanatory except for the following:

ALLOWABLE POWER FLUID OUTLET TEMP is an arbitrary temperature, lower than the desired outlet temperature, used to calculate the amount of auxiliary heating necessary if the design criteria were relaxed to this point.

STABILIZATION PERCENTAGE is the percentage difference between storage input and output over a complete cycle which must be achieved before the program will stop. At this point the unit is assumed to be repeating itself each day.

NUMBER OF DAILY CYCLES is the maximum number of cycles the program will be allowed to run.

2. Element Analysis Subroutine Data (Fig. D-1)

Definitions :

AEL: The total area in $\mathrm{m}^{2}$ of inside surface of the transfer fluid heat exchanger in contact with storage medium. 
AEP: The total area in $\mathrm{m}^{2}$ of inside surface of the power fluid heat exchanger in contact with storage medium.

ADL: The total area in $\mathrm{m}^{2}$ of common direct contact between the power fluid and transfer fluid heat exchangers.

DEFF: The effective diameter in meters of one double tube of heat exchanger upon which storage medium solidifies (the diameter of a circle whose perimeter is that of a cross section of a touching power fluid and transfer fluid tube).

XLEFF: The effective length in meters of one tube of heat exchanger.

XNT: The number of heat exchanger tubes.

RHOM: The density in $\mathrm{kg} / \mathrm{m}^{3}$ of storage material.

HTCS: The heat transfer coefficient of subcooled power fluid in $\mathrm{kW} / \mathrm{m}^{2}-{ }^{\circ} \mathrm{K}$.

HTCV: The heat transfer coefficient of power fluid vapor in $\mathrm{kW} / \mathrm{m}^{2}-{ }^{\circ} \mathrm{K}$.

3. Thermkeep Properties Subroutine Data (Fig. D-I)

In addition to the DATA section, two functions are modified directly for each run: $\mathrm{HL}(\mathrm{WL})$ and $\mathrm{HT}(\mathrm{X})$. HL(WL) computes the heat transfer coefficient of the transfer fluid as a function of flow rate in $\mathrm{kJ} / \mathrm{sec}-\mathrm{m}^{2}-{ }^{\circ} \mathrm{K}$. For this function the tube size, the fluid properties and a helix diameter must be known. $H T(X)$ computes the heat transfer coefficient of vaporizing power fluid as a function of quality in $\mathrm{kJ} / \mathrm{sec}-\mathrm{m}^{2}-\mathrm{O}_{\mathrm{K}}$. 
PLAL HEAT EXCHANGFR - MEAT OF FUSION THERMAL STJRAGE ANALYSIS

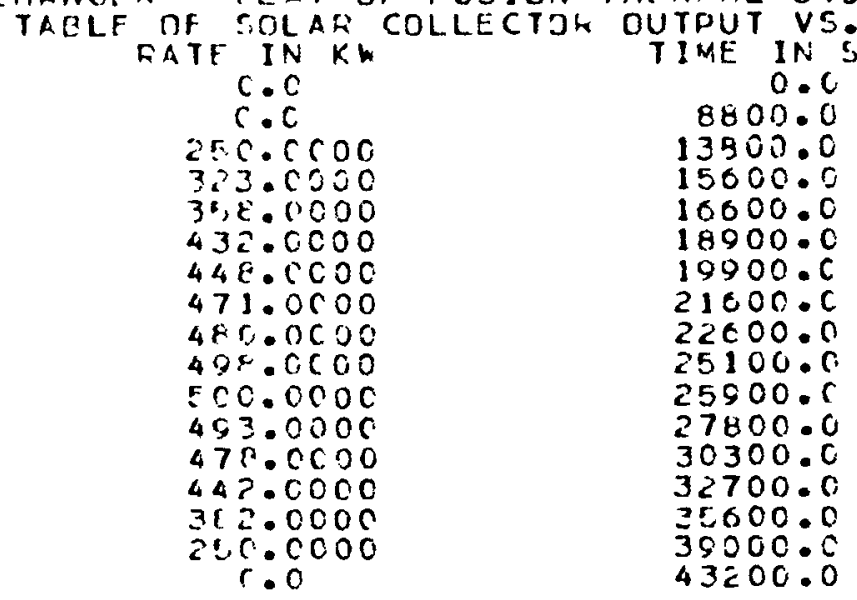

MAIN PRDGHAN DATA

TFANSFER FLUIC STECIFIC HFAT, KJ/KG/K

TEANSFER ILUIC IHLFT IF HDERATURL. K

PCITR FLUID SF.HT.LIQUID. KJ/KG KK

PCWEF FLUIL HEAT/VAP. KJ/KG

PCWER FLUID SE.HT.-VAROI. KJ/KG/K

PCIKLR FLUID SATUPATIOH TEMPLRATURE . K

PTIER FLUID INLET TEMDEIATURF $X$

DE SIRER POWER FLUJT JUTLIT TEMP. K

ALLCWAELE FOWER FLUID GUTLET TEMP. K

PCWER FLUID FLOK KATE. KG/SEC

IRISOLATICN STAFTING TIMT. SEC

QEGIN NET CHAFGE SEC

ERD NET CHARGE. SEC

THERMKFEP SPFCIFIC HEAT. KJ/KG/K

NUNEEF OF ELEMLNTS FJK ATALYSIS

ANCUNT OF STOFAGE MATIFIAL. KG

MININUN TINE INCFIMENT. SEC

MAXIMUN IINE INCFEMENT. SEC

MAXIMUM TRANSFER FLUID FIIIN, KG/SEC

STATILITATION PEECENTAGE

NUNET F OF DAILY CYCLES

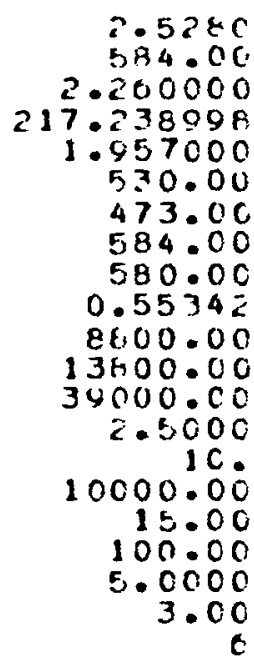

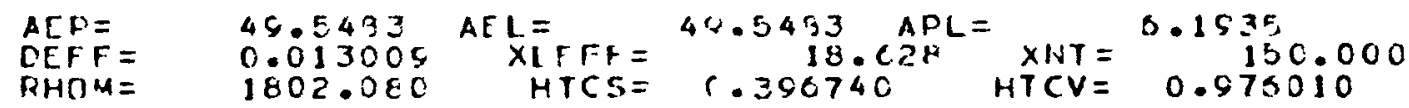

TAELE DF TEMPERATURF. CR.THALPY. AND SOLID FRACTION OF THETMKEEF $T(1)$ H(1) i( 1 )

373.00
505.00
528.00
530.00
535.00
541.03
544.59
549.17
557.86
500.00
563.00
573.00
583.00
643.00

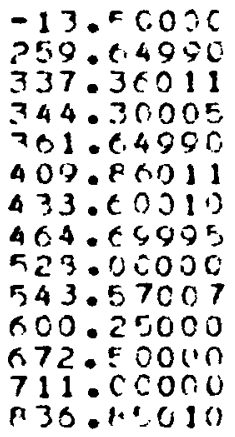

1.000000

1.000000

1.000000

0.700000

0.736200

0.570000

0.615000

C. 590 000

C. 400000

0.388700

0.347500

0.255000

0.0

Figure D-1. Dual heat exchanger program input data. 
Input Data Selection

Much of the input data does not change from run to run. The insolation table, Thermkeep properties, and all the main program data remain constant except for the Number of Elements for analysis (normally 10 for general use, and 25 for more accurate final designs) and the amount of storage material. The insolation table is a copy of the "Albuquerque Winter Mean Solar Day" modified to 12 hours by omitting a turbine overnight idle period. The Thermkeep properties are "Table 10 " for Thermkeep determined by the work described in Part II. The remaining data applies to toluene as the power fluid and the system characteristics as required by this contract. The maximum and minimum time increments were determined by trial and error during the early stages of program development.

The element analysis data were chosen with the help of a desk top computer. First, desired heat exchanger sizes, areas, and tube diameters are arbitrarily selected. Then the number and length of the tubes are varied until a combination is found which produces about a 10 psi drop in the transfer fluid exchanger and a reasonable drop (20-30 psi) in the power fluid exchanger. Then an amount of storage material is arbitrarily selected. If the height-to-diameter ratio is specified (normally 1.0 ), this produces a helix diameter of the tubing. From the known mass flow of power fluid and heat exchanger geometry, the heat transfer coefficients of the power fluid are now calculated.

The effective diameter is calculated by using the diameter of a tube whose perimeter is the same as the sum of the two fluid tubes' perimeters minus the section which they have in common. This report uses all equally-sized tubes for both power and transfer fluids and the common area was $11 \%$, or $40^{\circ}$ of arc on each tube in direct contact (Figure D-2). A small addition to the perimeter was made for weld fillet between the tubes.

Function HL(wL) receives the transfer fluid flow rate from other parts of the program and returns a heat transfer coefficient. This function is modified for each run because it uses the tube inside diameter and helix diameter which change with each case. The helix diameter is calculated as the diameter of one heat exchanger coil such that one-half of the storage material is enclosed by the coils and one-half lies outside them. 


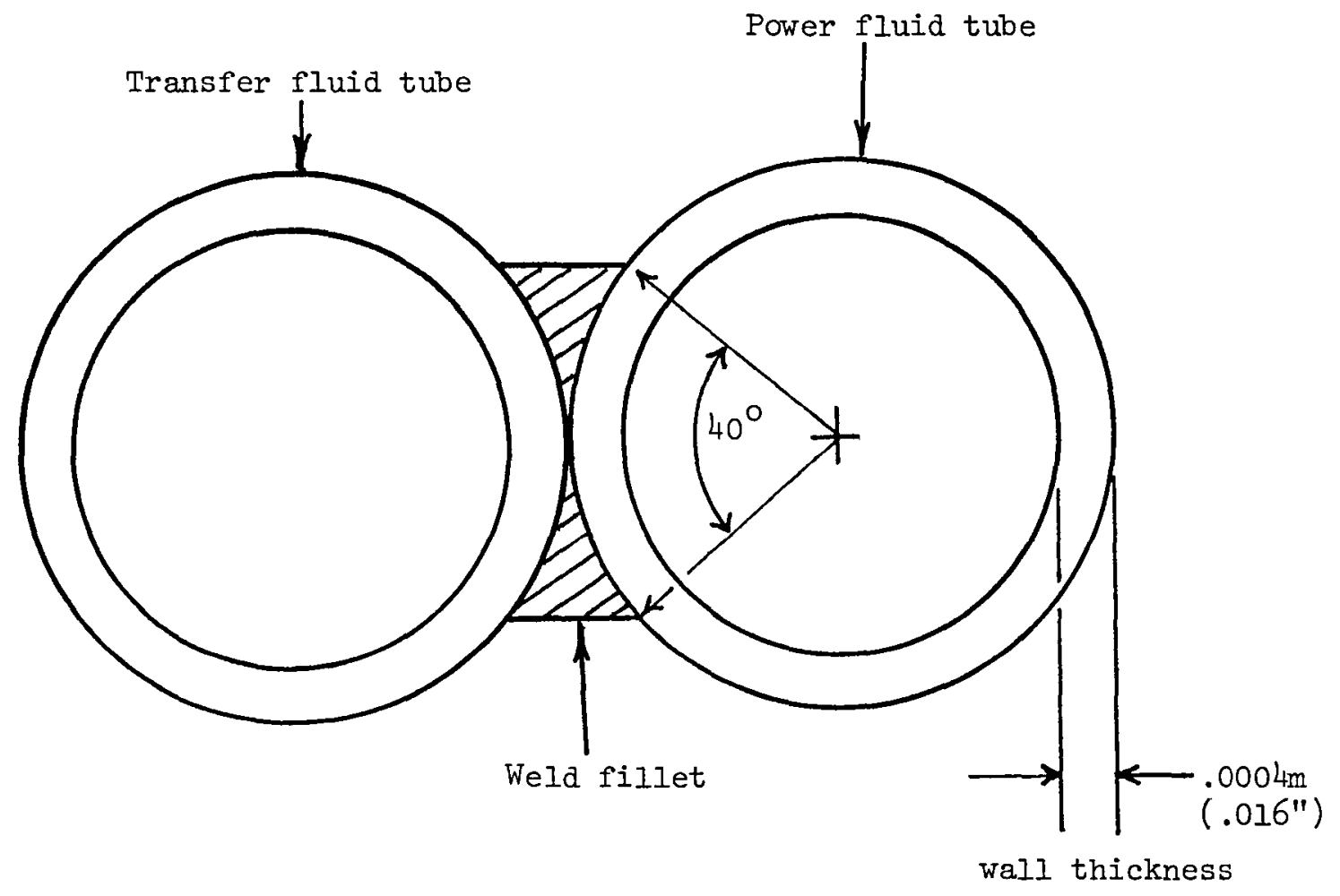

Figure D-2. Typical dual heat exchanger tubing. 
From the heat exchanger geometry and transfer fluid properties, the following coefficients are determined:

CRE: A multiplier of the mass flow rate which will give the Reynolds number

$C R E=d_{t} /($ fluid area $x$ fluid viscosity $)$

where $d_{t}$ is the tube inside diameter.

RECRIT: The critical Reynolds number

$$
\operatorname{RECRIT}=2100\left[1+12\left(d+d_{h}\right)^{0.5}\right]
$$

where $d_{h}$ is the helix diameter.

CHTC: A heat transfer coefficient factor:

$$
\mathrm{CHTC}=\left(.022 \times \mathrm{C}_{f} \times \mathrm{Pr}^{4}\right) / \mathrm{d}_{\ell}
$$

where $C_{f}$ is the fluid thermal conduc-
tivity.

QX: A dimensionless factor used in the calculation of the effect of the helix diameter on heat transfer in laminar flow:

$$
Q X=\left[\left(\frac{d_{t}}{d_{h}}\right)^{2} \quad \operatorname{Pr}\right]^{\frac{3}{4}}
$$

HTCI: The heat transfer coefficient for turbulent flow:

$$
\text { HTCI }=\text { CHTC } \times \operatorname{Re}^{\cdot 8}
$$


Function $H T(X)$ receives the power fluid quality and returns a heat transfer coefficient. The relationship for toluene is roughly a sigmoidal shaped curve, of which a linear approximation is a good average. Therefore, during this contract we used

$$
\mathrm{HT}(\mathrm{X})=\mathrm{X} \mathrm{x}(\mathrm{HTCV}-\mathrm{HTCS})+\mathrm{HTCS}
$$

\section{Output Data}

For each case run, a back-up calculation print-out was made. An example is shown in Figure D-3. Cost calculations are based on the actual cost of a prototype storage unit, and integrate manufacturing and material costs both on a surface area and weight basis. The only difference from case to case is in the tube material cost, where there is a lower cost by weight as tubing gets larger.

A dollar amount for each category is then printed out, along with a unit total cost. As a point of interest, the percentage of total cost of the heat exchanger, insulation, cover, tank and storage material are also listed.

Next, the geometry and weight input data are tabulated, and the resulting dimensions and weights are listed (Figure D-4).

Finally, the heat exchanger dimensions are calculated and listed along with Reynolds numbers, heat transfer coefficients, and pressure drops. These outputs allow us to check total surface area and to vary the number, length, and diameter of tubes in order to produce desired pressure drops. With toluene, we always found that it was in laminar flow in the liquid region, turbulent flow in the vapor region, and the vast majority of pressure drop was due to the vaporization acceleration. Also calculated were the power fluid pressure drop assuming that it was liquid through the entire tube, and vapor pressure drop assuming it was vapor for $90 \%$ of the tube -- both of these cases were assumed extremes. 


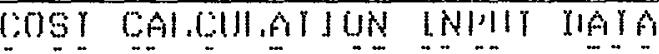

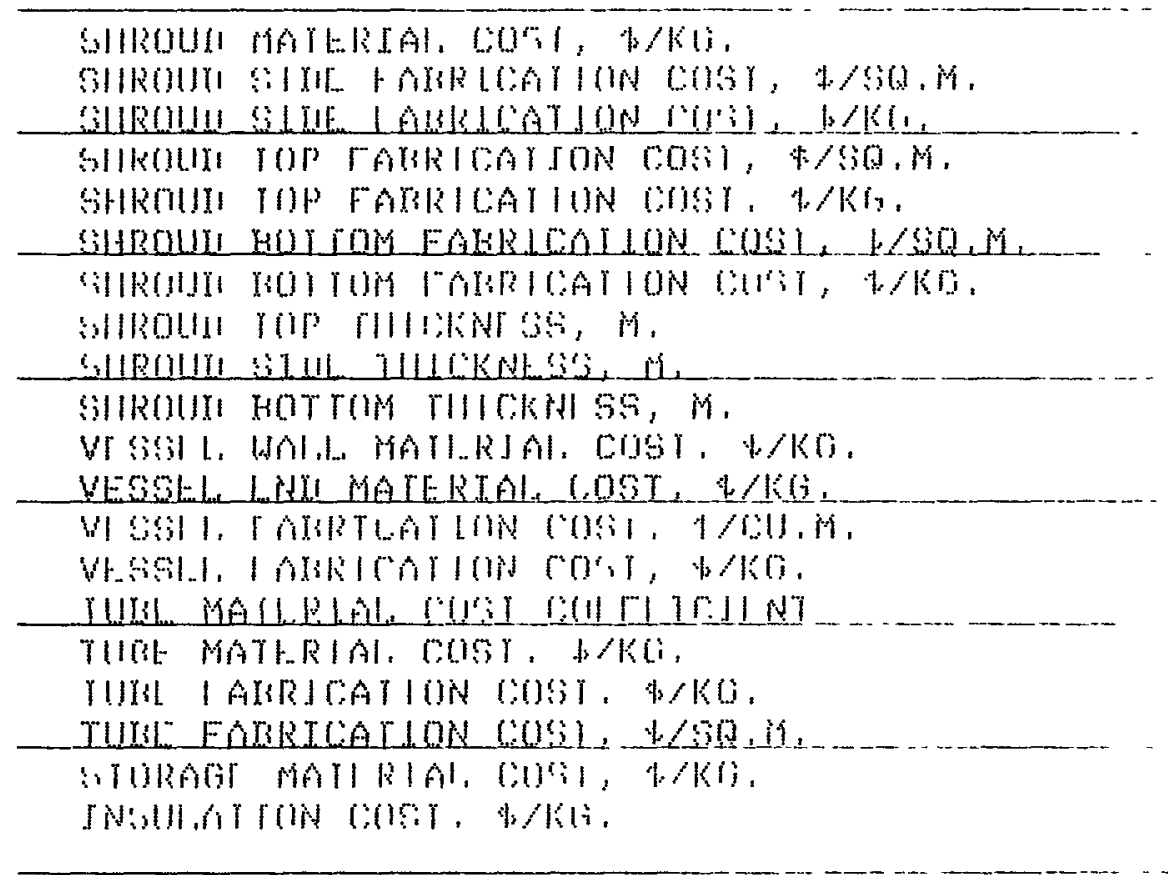

$\therefore 04$

10,011

1, 0,33

76.35

$1,9: 3$

$9, .64$

1.61

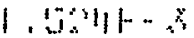

1.210.2

$3.40 .36 L^{2}-3$

4718

.4806

$61 ! 1,11$

3.1197

.923

$x, 9091$

11.9

964.3398

, 441

$\therefore 26$,

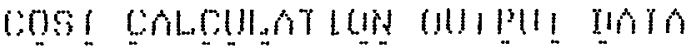

\section{IIIII BOCIRLAL COSI, *}

IUTAL, TUHE $6019 \mathrm{~T}, 1$

BTORAGE MATHRIAL, COSY, क

CNSULAEITUS COOSI, \%

SIRUIU SJII MOItRIAL [UST, 1

GIROUI SU rutal, $6.09 \%$, 非

1179

$114<3$.

41110 .

07.3

13,16

sobs.

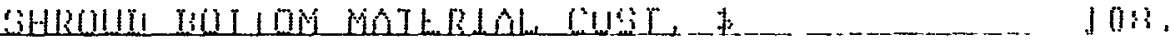

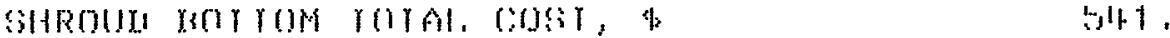

SHROUIT TOP MOIFEIAI, COST, : 46.

GllROUIII TOP TOTAL DOSBY, b.

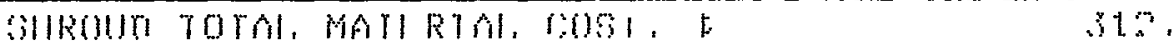

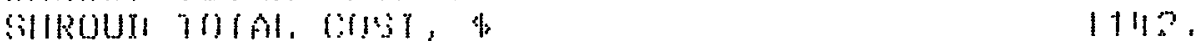

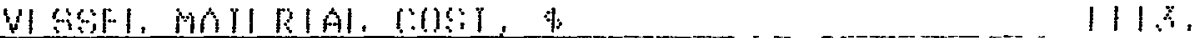

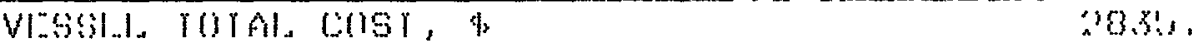

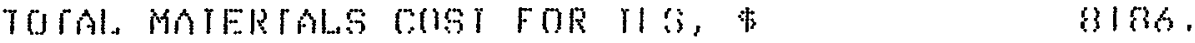

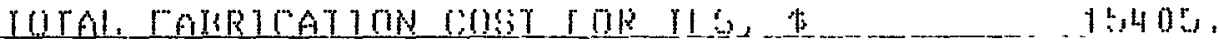

ता iOTn, CUST, \$

23091

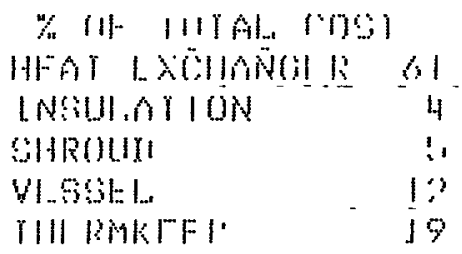

Figure D-3. Cost calculations print-out example. 
OCOME IRY ANO WUI.1OUI JWPUT IHAIA

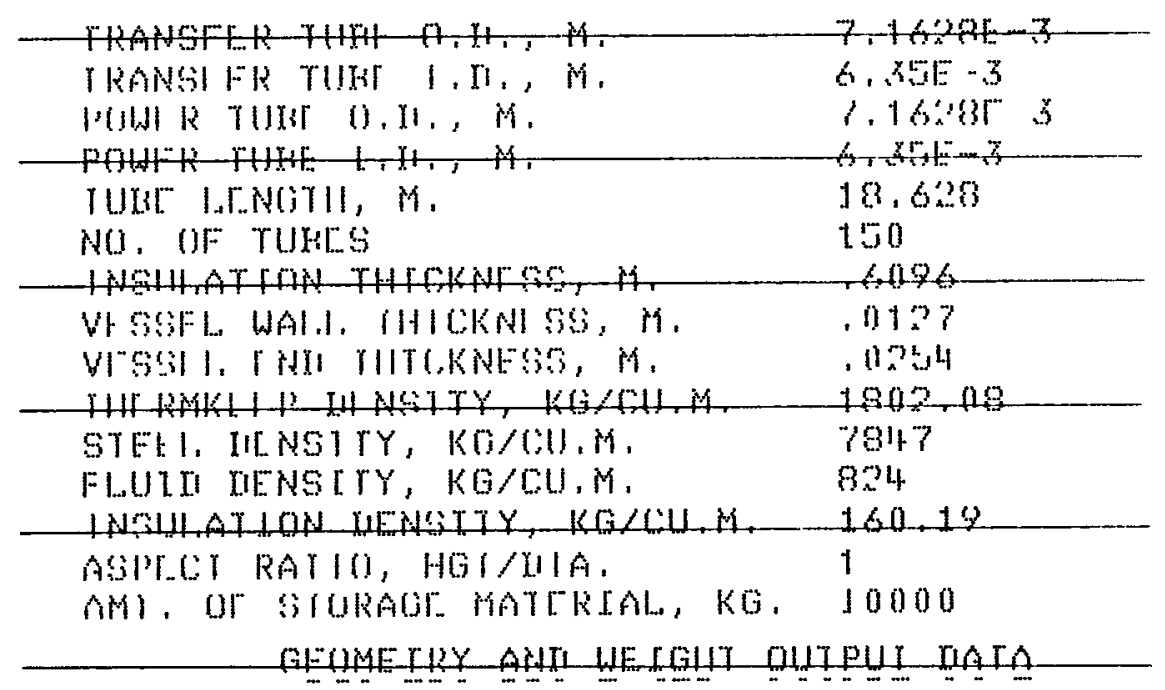

\begin{tabular}{|c|c|}
\hline 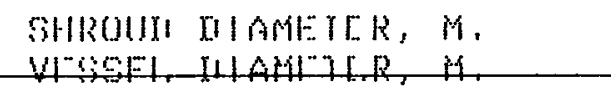 & $\begin{array}{r}3.164 \\
1.2310 \\
\end{array}$ \\
\hline 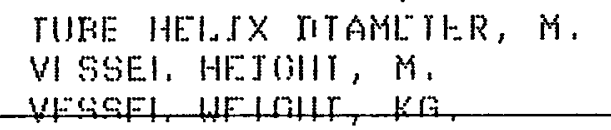 & $\begin{array}{l}1115 \\
1.9310 \\
23.36 .6501\end{array}$ \\
\hline 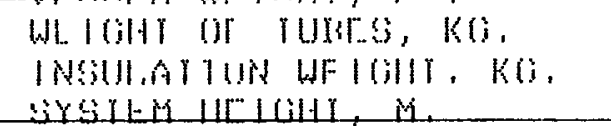 & $\begin{array}{l}310,2771 \\
30414.427 \\
3,2018 \\
\end{array}$ \\
\hline $\begin{array}{l}\text { SYSTEM WF } 1 \text { OHH, KO, } \\
\text { POUER TUME LII". }\end{array}$ & $16371.111 \% 20$ \\
\hline 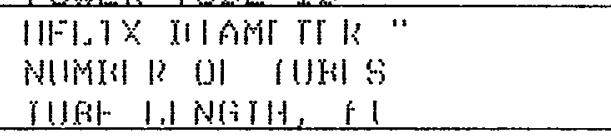 & $\begin{array}{l}11,391 \\
150 \\
6,1.11,1,3\end{array}$ \\
\hline $\begin{array}{l}\text { YUHL TNSIIIL ARLA, HIO } \\
\text { rURF INSIII ARTA, W? }\end{array}$ & 600 \\
\hline 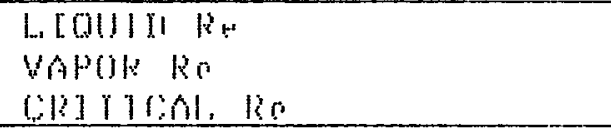 & $\begin{array}{l}609 \\
56091 \\
3113\end{array}$ \\
\hline $\begin{array}{l}\text { HTCl, lilu/hr i } 1 \mathrm{a} \text { of } \\
\text { HICl, } \mathrm{kW} / \mathrm{m} \text { ? } \mathrm{K}\end{array}$ & $\begin{array}{l}69.89579 \\
.39674 \\
\end{array}$ \\
\hline 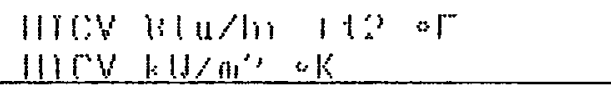 & $\begin{array}{l}171.91957 \\
.476111 \\
\end{array}$ \\
\hline $\begin{array}{l}A P \text { Accolarilion PSl } \\
A P \text { lignid whole labe rof }\end{array}$ & $\begin{array}{l}18 . .5 \\
.48\end{array}$ \\
\hline 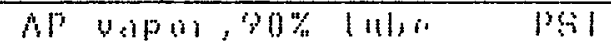 & $1 \cdot 3$ \\
\hline
\end{tabular}

Figure D-4. Output data print-out example. 
The FORTRAN program prints out all the input data as previously mentioned. Then the unit starting conditions are calculated and listed -- the starting temperature, starting solid fraction, and total unit energy. The charging fluid flow rate and both fluid outlet temperatures are printed each half-hour of elapsed time.

A map of the unit showing the temperatures of storage medium and both fluids, along with fluid quality and solid fraction of the medium in each element or "slab" is printed out at two points in each daily cycle -- at the beginning of net charge when the unit is at its lowest state of charge, and at the end of charge when the unit is at its highest state of charge. Also the total unit energy at these times is printed out, and the difference between them, once the unit is stabilized, is equal to the amount of energy actually output from storage, and does not include the heat transferred directly from the transfer to the power fluid.

At the end of a daily cycle, a summary of the energies transferred is printed:

The LOST COLLECTOR HEAT is the amount of heat available but unused because of maximum flow conditions.

The STORAGE OUTPUT is the total amount of energy which came out of the unit in the power fluid stream.

The STORAGE INPUT is the amount of energy which entered the unit in the transfer fluid stream.

The AUXILIARY INPUT is the amount of heat required to raise the power fluid outlet temperature to the Desired Outlet Temperature.

The DEFICIT REQUIREMENT is the amount of heat required to raise the power fluid outlet temperature to the Allowable outlet Temperature.

The EXCESS HEATING is the amount of heat which exited the unit above the desired outlet temperature. 
The BOILER REQUIREMENT is the total daily boiler consumption.

Also listed are the total collector output, the elapsed time, the number of time steps used during this cycle, and the cycle ending total energy.

At this point, a new cycle is begun; or, if the unit has stabilized, the program stops. 



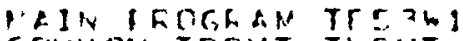

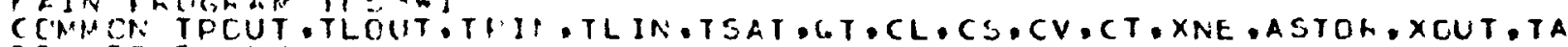
- IF H. TE, TO.NC

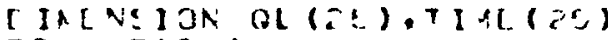

160 FCHPAT(IE)

$1: 1$ FrFHAT(FIC.4. T1C.1)

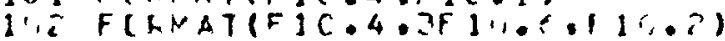

$10:$ F CFNAT(4F 10.3$)$

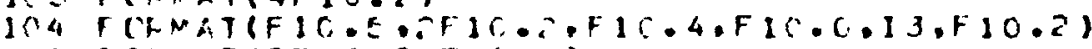

16 FCENTT(EF) $3.0 . F 1(.4)$

I: IC CMAT(ZF 10.2$)$

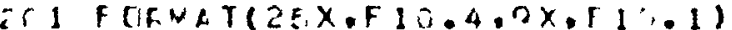

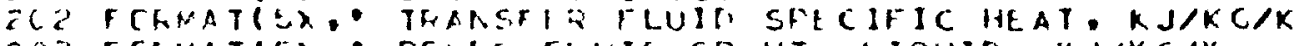

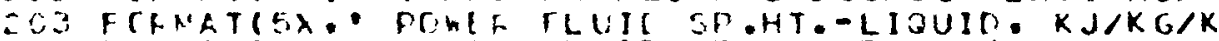

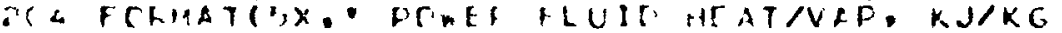

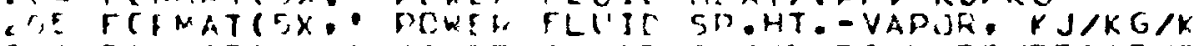

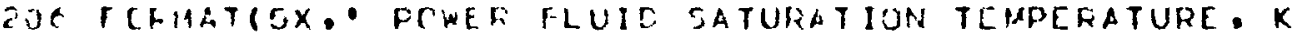

207 FFMATSEX.: THANSFC: FLUID INLET TEMPCRATURE. $K$

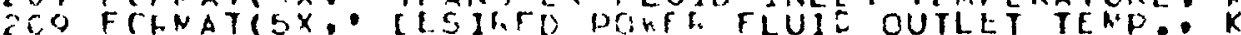

CCL FOFNATSE. FIMEh FLLI! INLFT THMPEKATURE - $K$

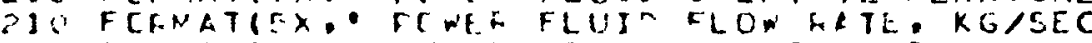

211 FCFMAT(5X. IISGLATILH STARTIH.G TIME. SEC

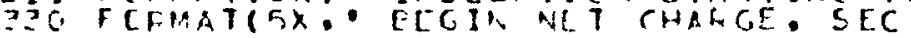

331 TCKMATLEX. END MET CHBSTE, SEC

-12 FCFNATSEX. AlOJURT IF STORAGE NATERIAL. KC,

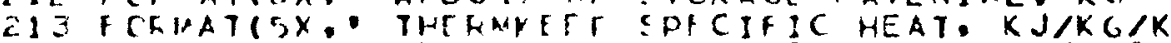

214 FLIMATISX. MUNPER TF TLIMEHITS FOR AHALYSIS

c1E FLFMATKEX. NUIE? OF TAILY CYCLES

E14 FCFNATREX, STAEIIIZATITN PEFCIHTAGE

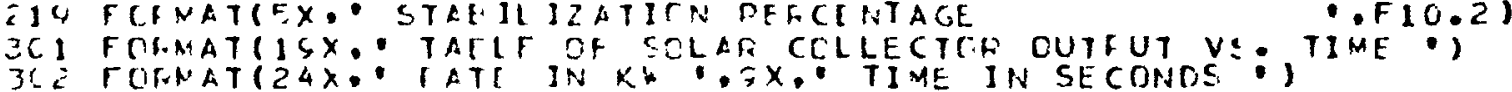

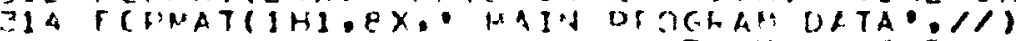

317 CRMATIIHI:5X: MUAL HETT EXCHENGEF - HEAT OF FUSION THERMAL STORA HCI ANALYS1S IS

Tle FCTMATUX:" NAXIMIN YINE INCFENENT. SEC

217 FCFAATLX. NIHIALM TINE INCFENENT. SEC

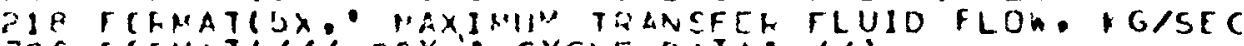

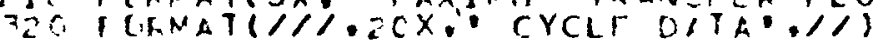

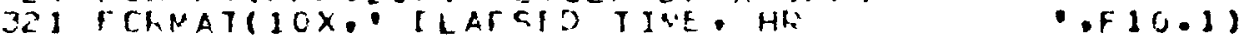

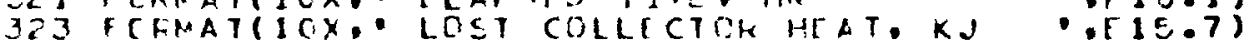

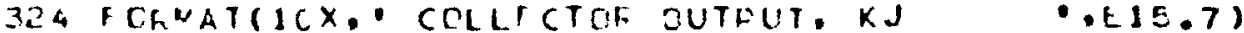

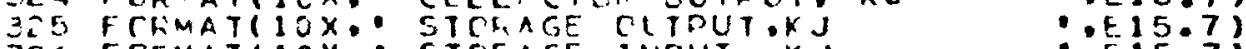

32E FREMATIIOX. STOFACE HUUUT KJ

327 TCEMATPIOX.: AUXILIARV INFUT.KJ :E 15.7 )

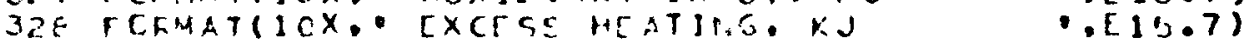

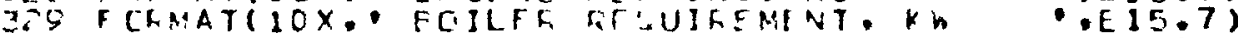

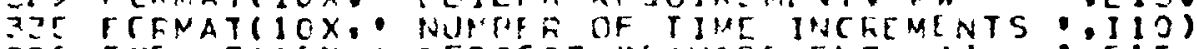

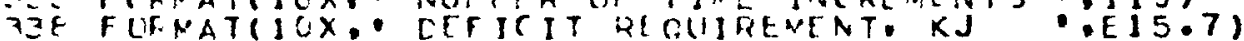

339 FCFNATIEX. ALLCWAGLE TOKER TLU1D OUTLET TEMP..K :.F10.2)

346 FCFMAT (FIC.4)

WF. ITE $(6.317)$

WFITE (E:301)

h.ITE $(\in, 3(2)$

Ft $A D\left(5.10() \mathrm{K}^{2}\right.$

CC $101=1, K 5$

K. E $\angle D(5.1 C 1)$ GL (I),TIMI. (I)

16 H.TE(e.201) CL(1). TIMf(I)

$T C=T$ INE (KE)

F. EAD $\left(* 10 \frac{2}{2}\right)$

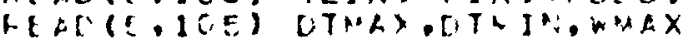

HAD $1: 1 C \theta)$ TE.TE

K. $1 T E(c .314)$

$\because F 10.2\}$

- F10.41

. F 10.4)

- F10.(.)

- F 10.6$\}$

- F (10.2)

$\because F(0.2)$

- Fio.a.

$\because$ F 10.5 j

- F10.2)

- F(0.2)

- F 10.21

- F $10 . \overline{2}$

- F10.41

- F10.0)

$.7 x .13 .1 / 18$ 


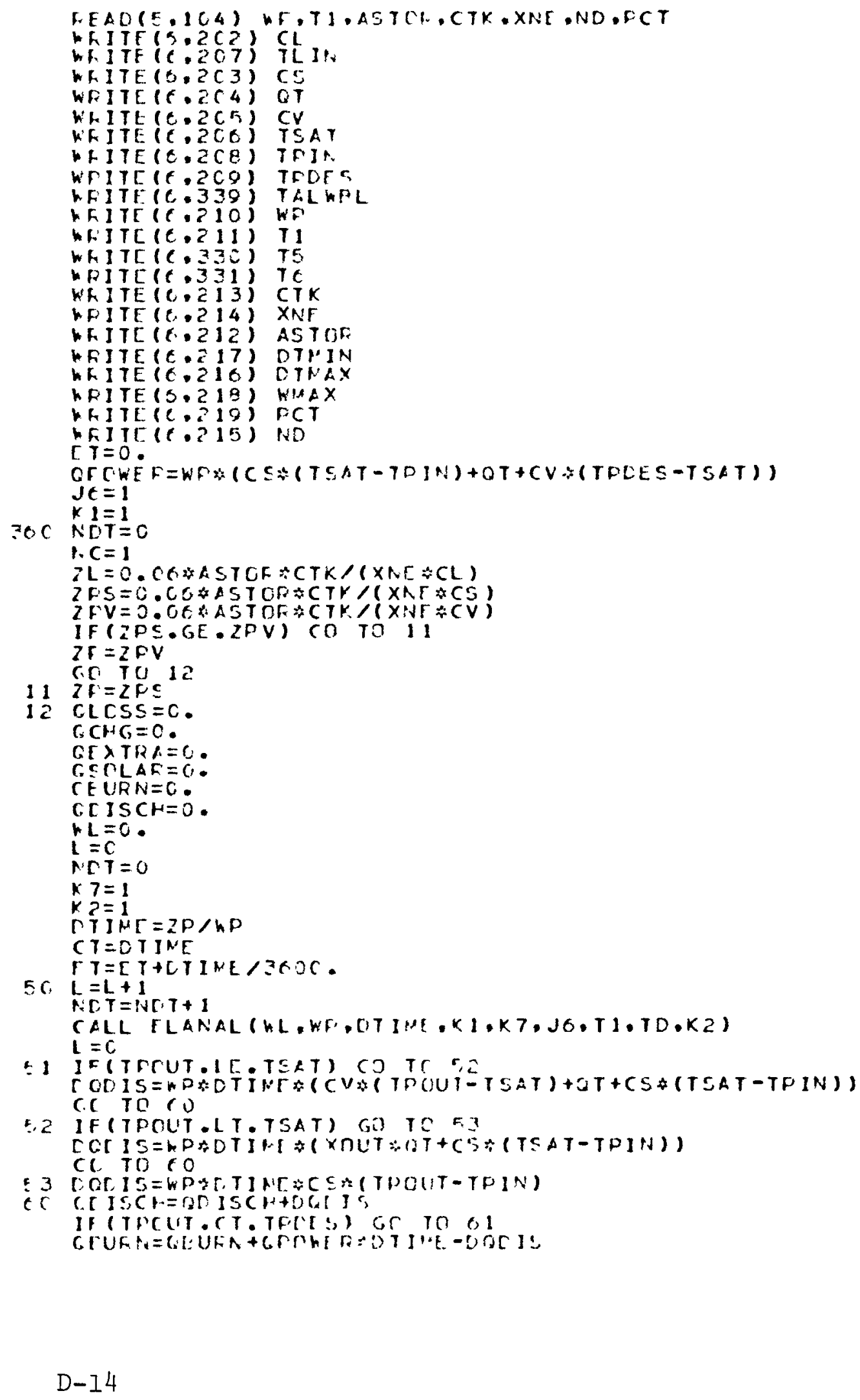


IF (TPCUT.CF.TALWFI.) GO TO Cí

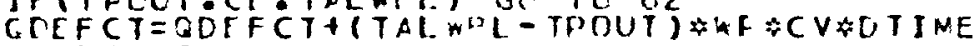

CO TO $C .2$

C 1 GTXTFA=GEXTFA+DCEIS-CDONER

C IF (K7.F O.1) GO Tr, 7 =

TCCHG $=R L * C L *(T L I P-T L C U T) * C T I N E$

C. $C H C=C C H G+D Q C H G$

CECL AF $=Q S C L A F+C_{*} C * \cap T I M F$

GLCSS =OLCSS+CCNCTIME-DGCHG

$7 C$ IF (CT.GE.T1) GO Iח AC

CTIME $=2 T / \mathrm{T} D$

IF (DTIME.LT.TTMIN) DTINC=DTMIN

IF (DTINE L LE:CTNAX) GC TG 7 I

TTIAE $=$ CTNAX

$71 C T=C T+D T I N F$

IF (CT.LE.TI) GC Tח $7^{*}$

C. TINE $=D T I N E-\left(C T-T_{1}\right)$

$C T=T 1$

$r z=2$

75. ET $=E T+D T I N E / 36 C C$.

K $7=1$

GQ TO

RU IF(CT.GE.TD) GC TO 20

IF $(K .7 .13 .1)$ GC TO 4 TC

$102 \quad 1=2.155$

IF(CT.GL:TINE(I)) GO TC?

$G(=Q L(I-1)+(C L(I)-G L(1-1):(C T-T 1 M E(I-1)) /(T I M E(I)-T I M E(J-1))$ C. 10 E 5

a' C C 1.T]PUE

[EF [T]MP=ZP/WF

If $(K 7 .[Q .2) \quad H I=C, /(C L:(T L: H-T A F P R))$

IF $(K 7 . F O .3) \quad K L=C . C /(C L *(T L I N-T L C U T))$

$K 7=3$

IF(KL.LE.KMAX) GD $10 \& 7$

$W L=W M A X$

a) DTIAL $=Z L / W L$

IF (DTINL.LT.DTIMF) GC T? 20

T.T IME $=D T I N P$

$G C$ TO FB

CC [TINL $=D T I R L$

C O TO E 8

$40 C C T=T 1+D T N A X$

ET $T=E T+D T M A X / 3 C C C$.

CT IME $=$ CT T A X

$7=2$

C C TO:O

IR IF (DTIME.LT.OTNIN) DTINE=DTMIIN

IF (DTTINE L $L \subset$ CTMAX) GC TO 91

CTINE = CTMAX

91 IF(CT.GE.TE) GO TS

$C T=C T+[, T] N E$

IF (CT.LE.T5) GO TR 416

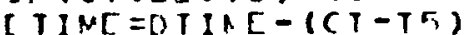

$C T=15$

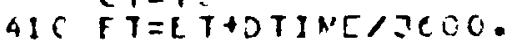

C.O TO 50

SE If(CT.CE.TO) CO TR 93

$C T=C T+O T I N E$

IF (CT.LE.TE) GO TO 423

DT TNE =OTINE $-\{C T-T A\}$

$C T=16$

4. C $T T=E T+D T I M / 3 G \cap C$.

GC TO EO 


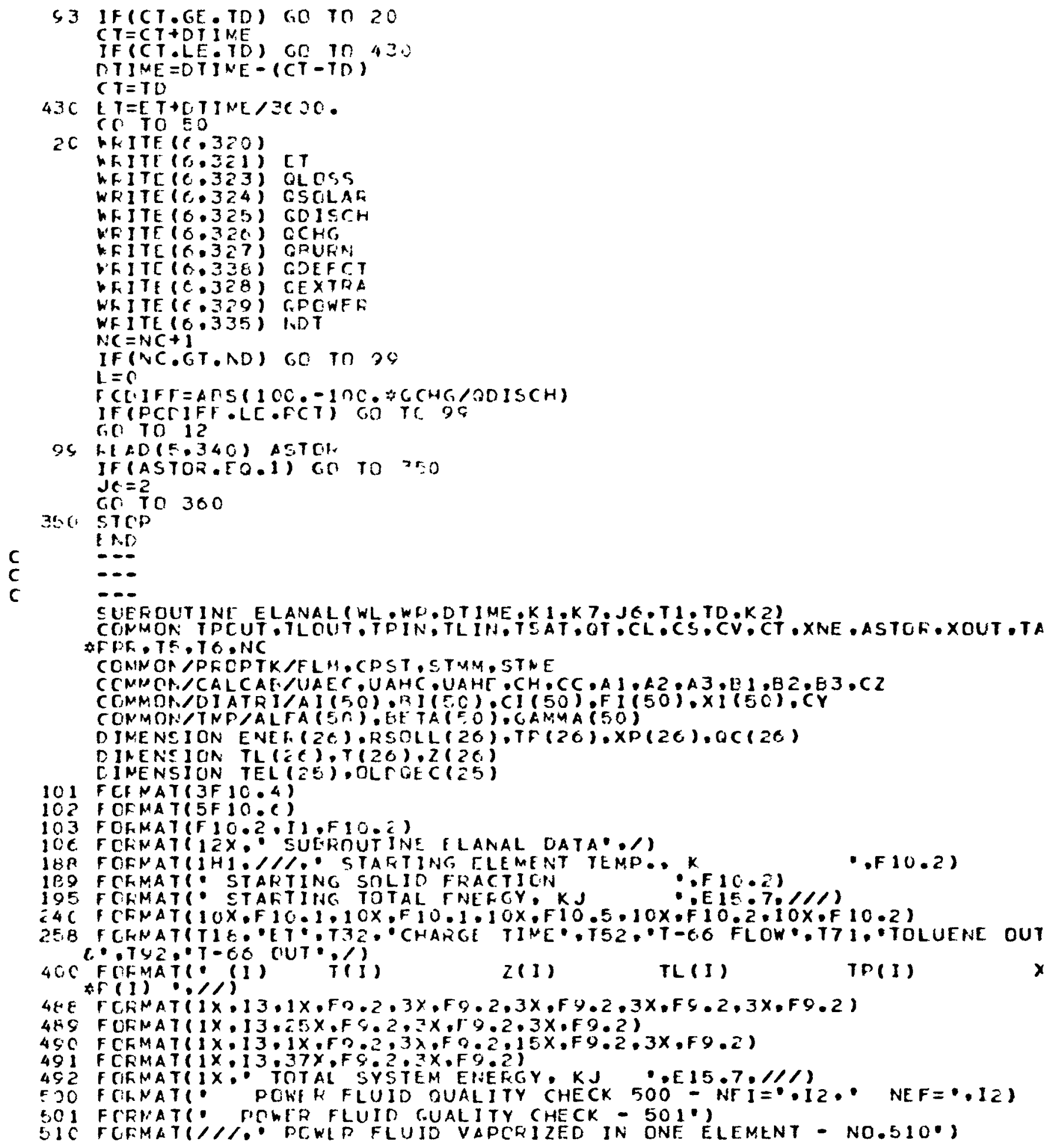




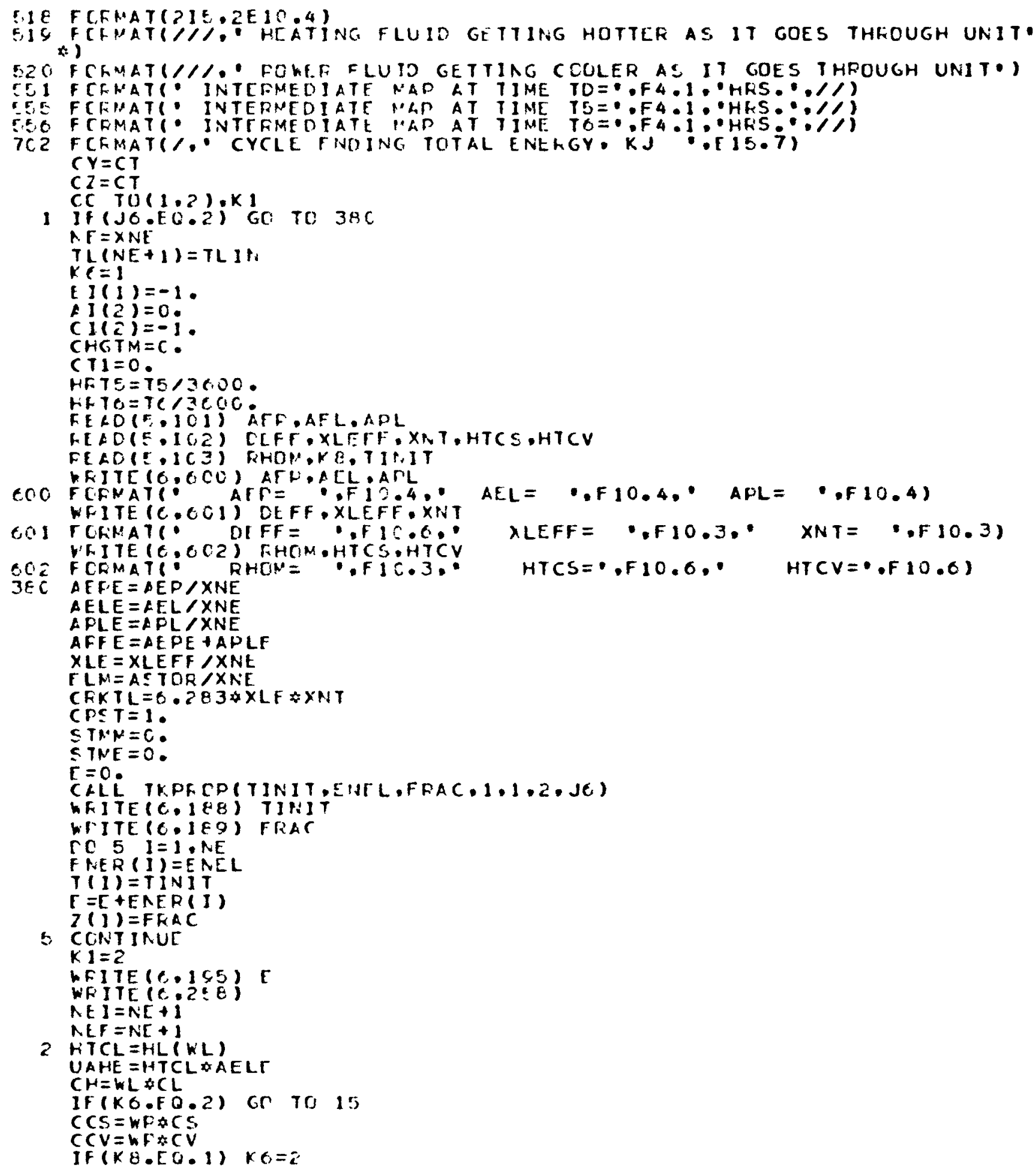




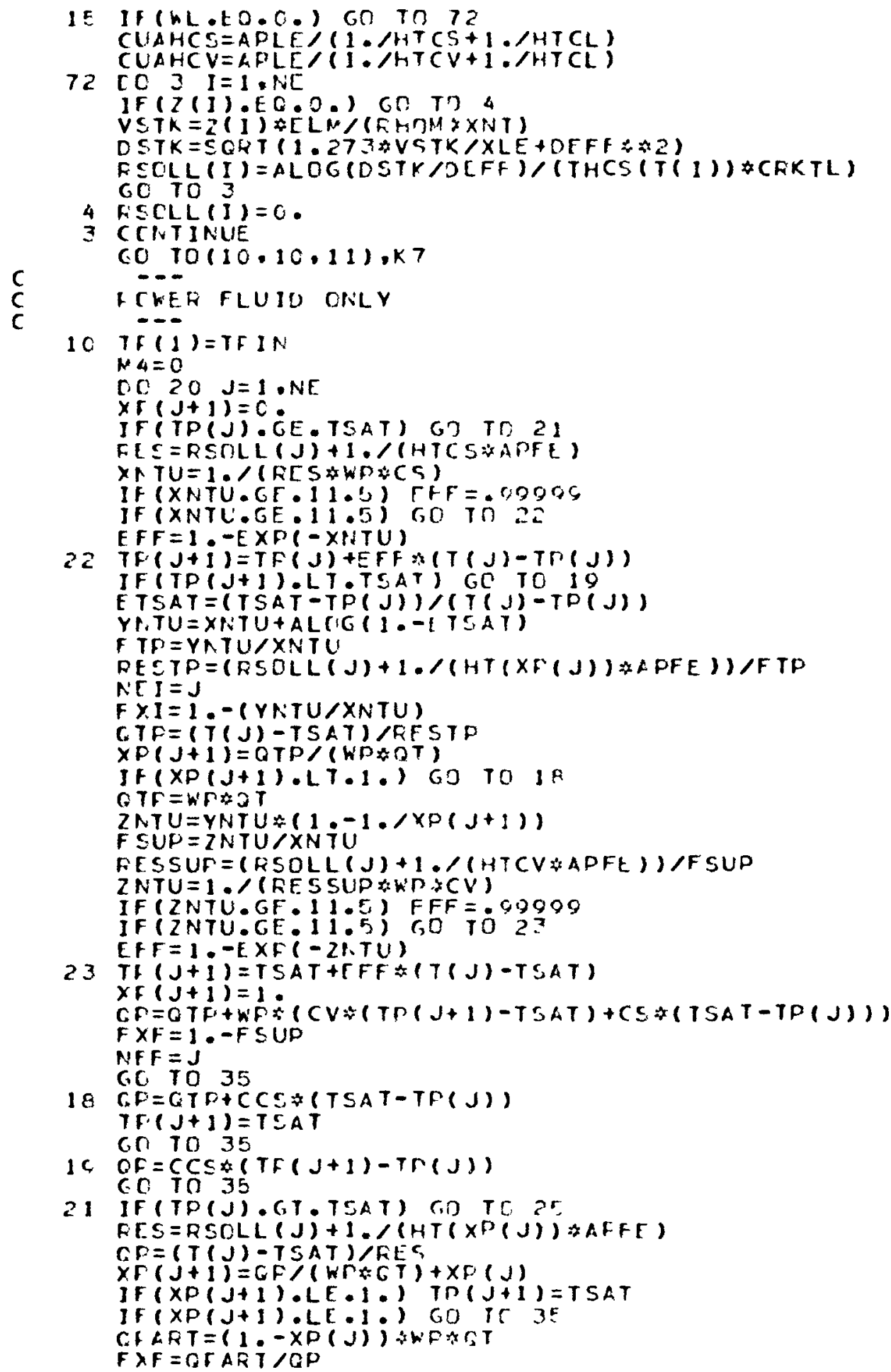




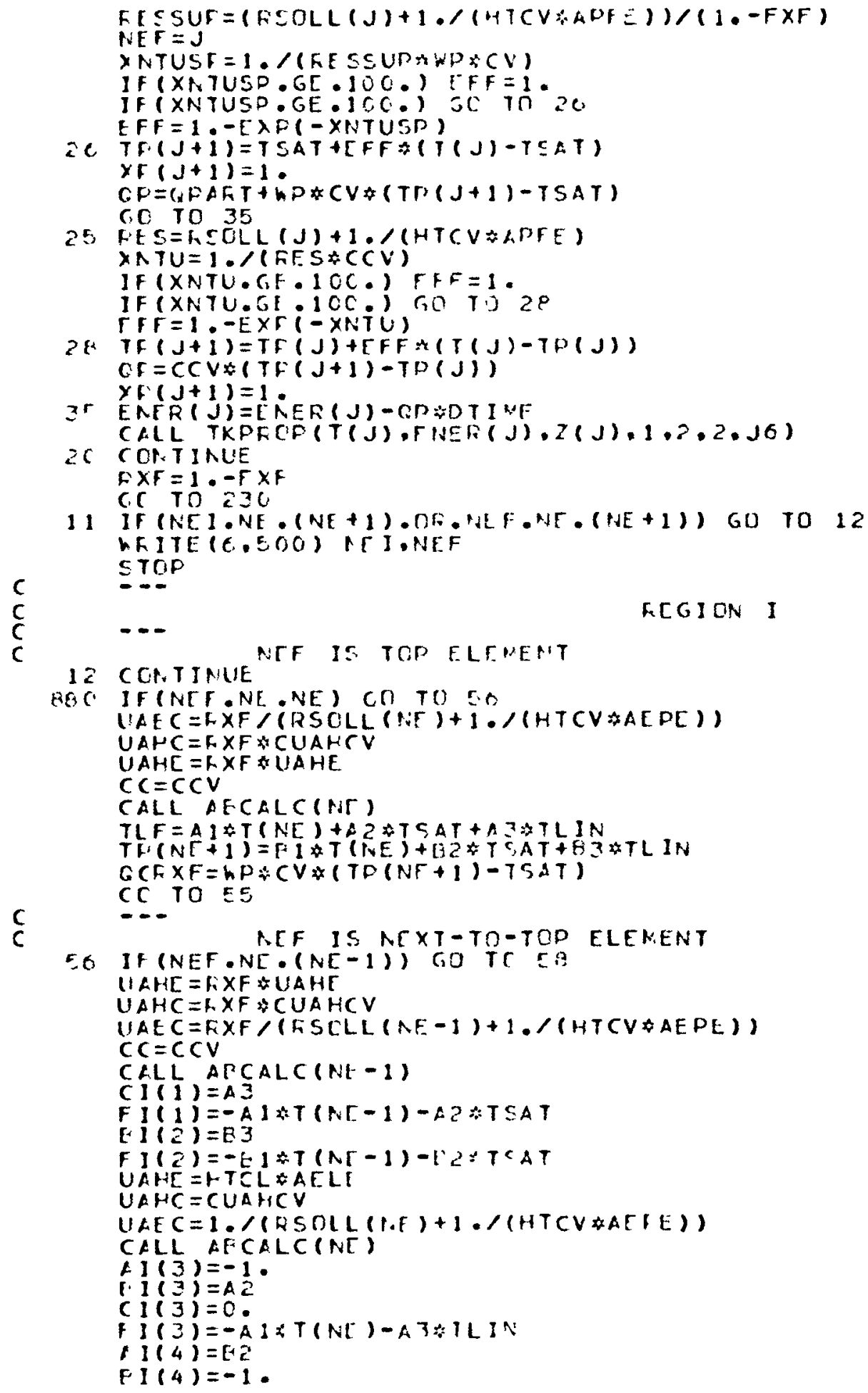

11 IF (NE I.NE - (NE +1). OF. NLF.NT . (NE+1)) GO TO 12 WFITE (E.EOO) A J ONEF

$c$
$c$
$c$ STOP

12 COATINUE

$-\cdots$

F[G]CN I

BQS IF (NTF. NE. NE) CO TO 50

(IAEC $=F \times F /(R S O L L(M A T)+1 . /(H T C V \# A E P C))$

UAHC $=F \times F$ CUAHCV

UAHC $=R \times F$ UAHE

$C \mathrm{C}=\mathrm{CCV}$

CALL AECALC(NT)

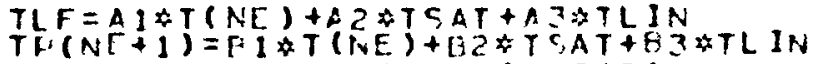

$G(F \times F=h P * C V *(T D(N F+1)-T S A T)$

C CCTO 


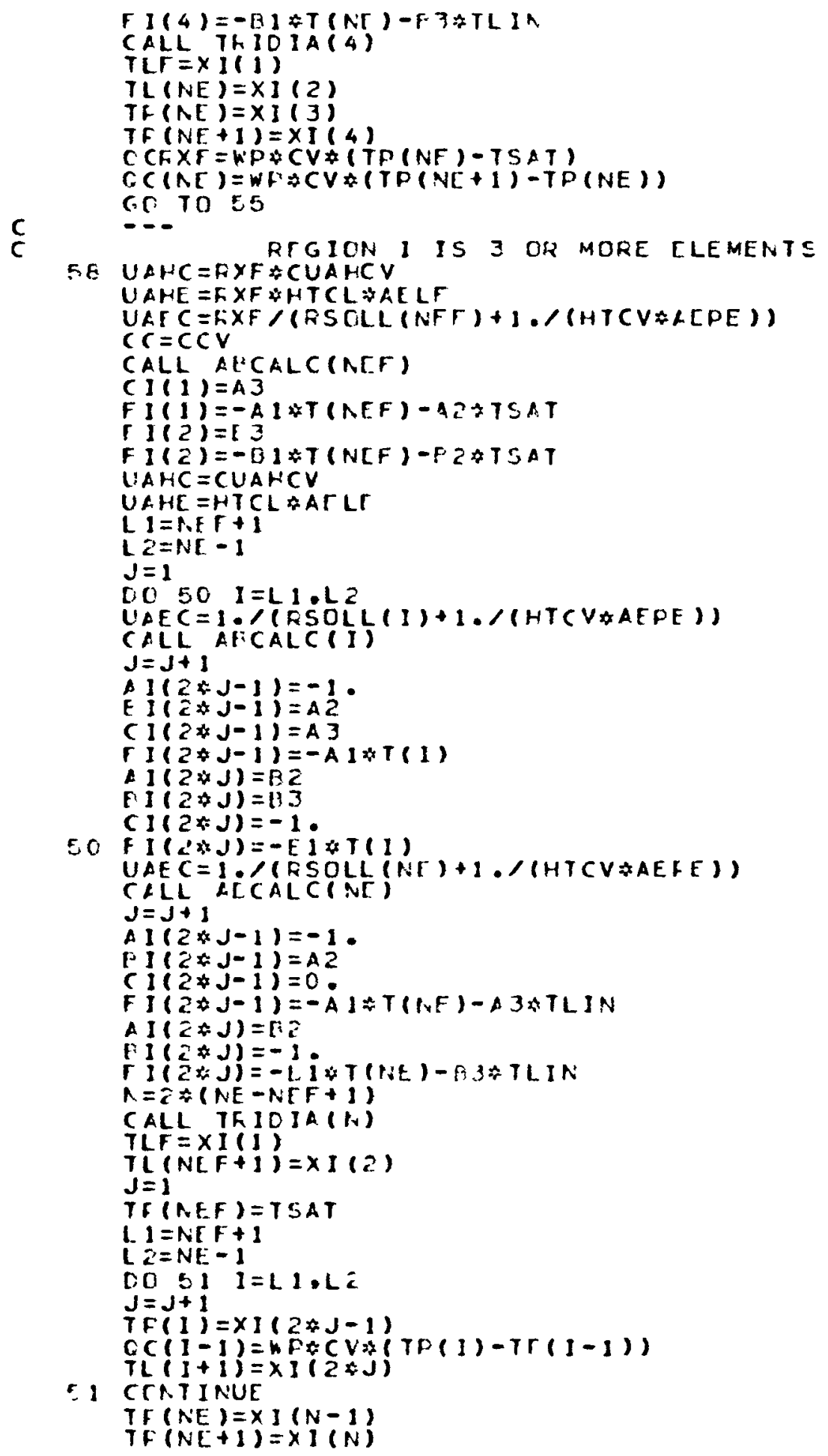




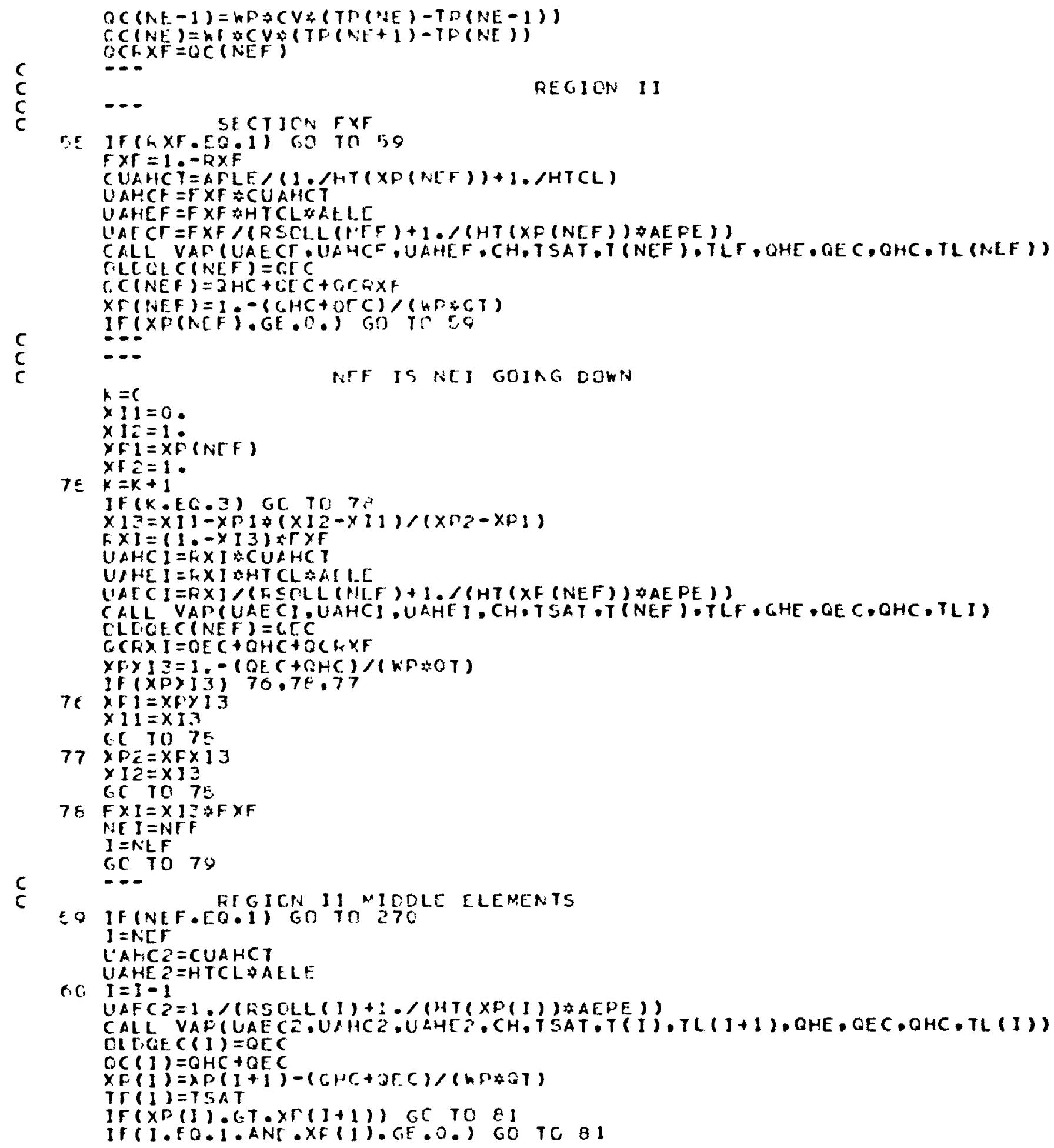




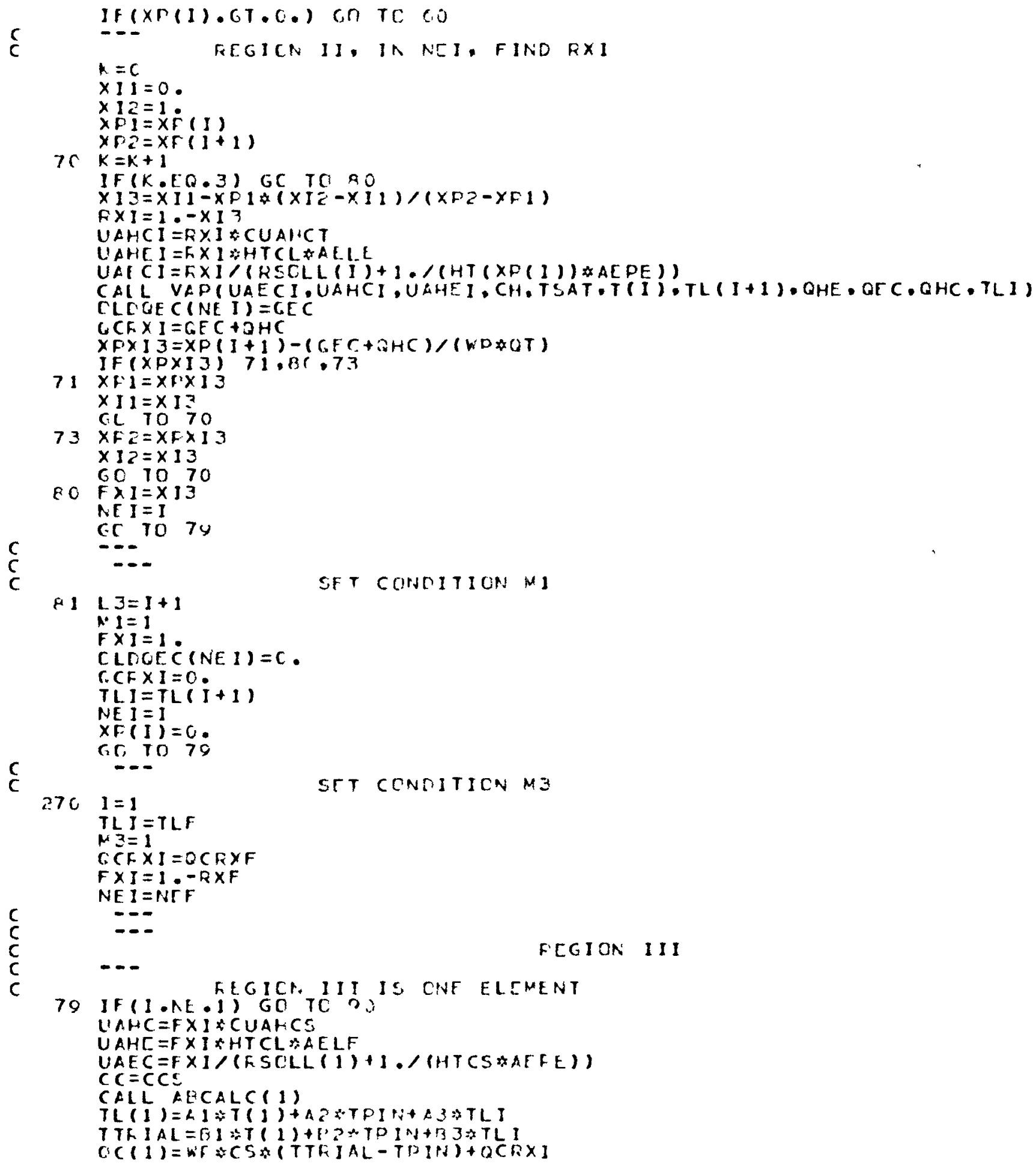




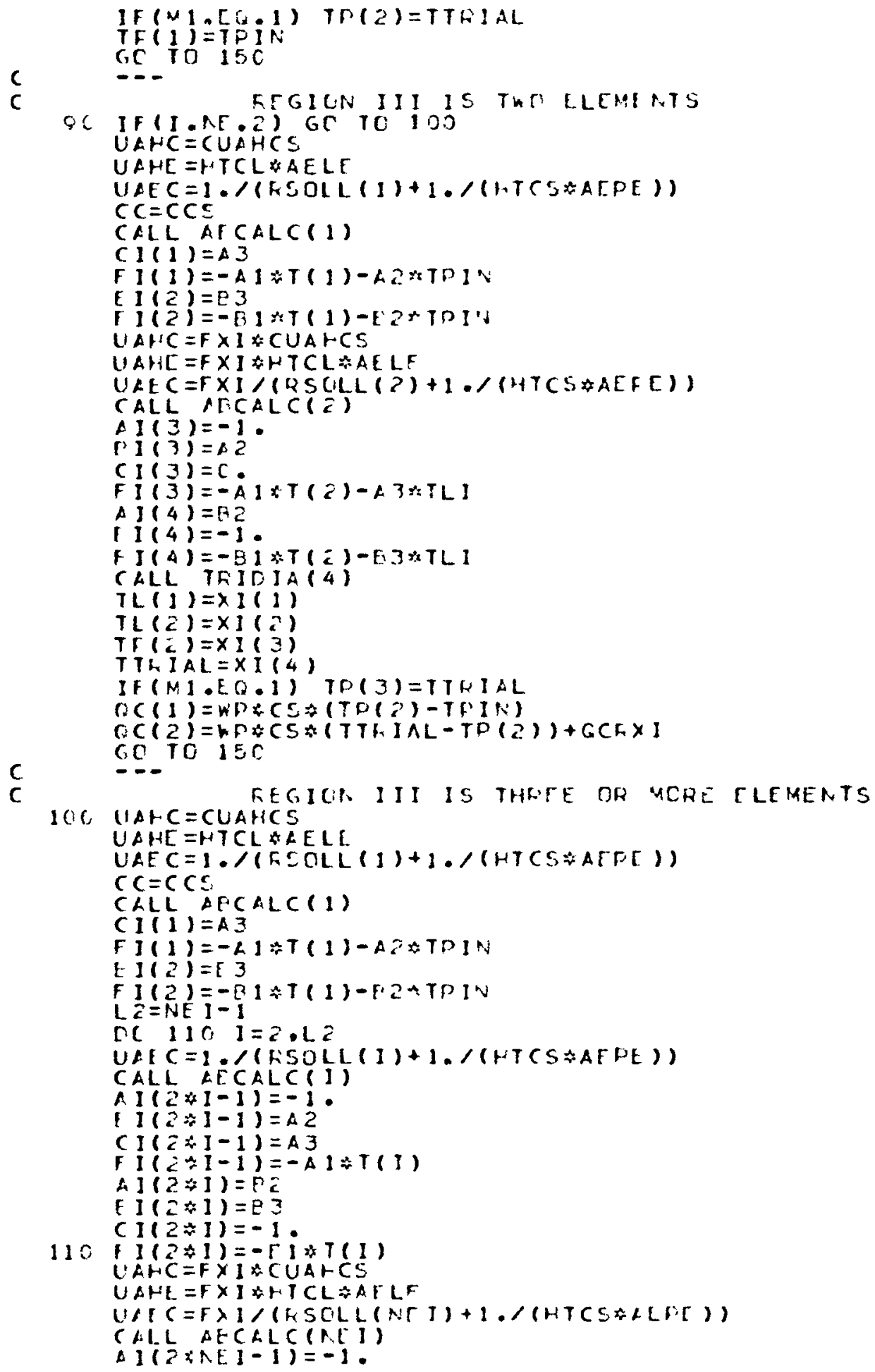




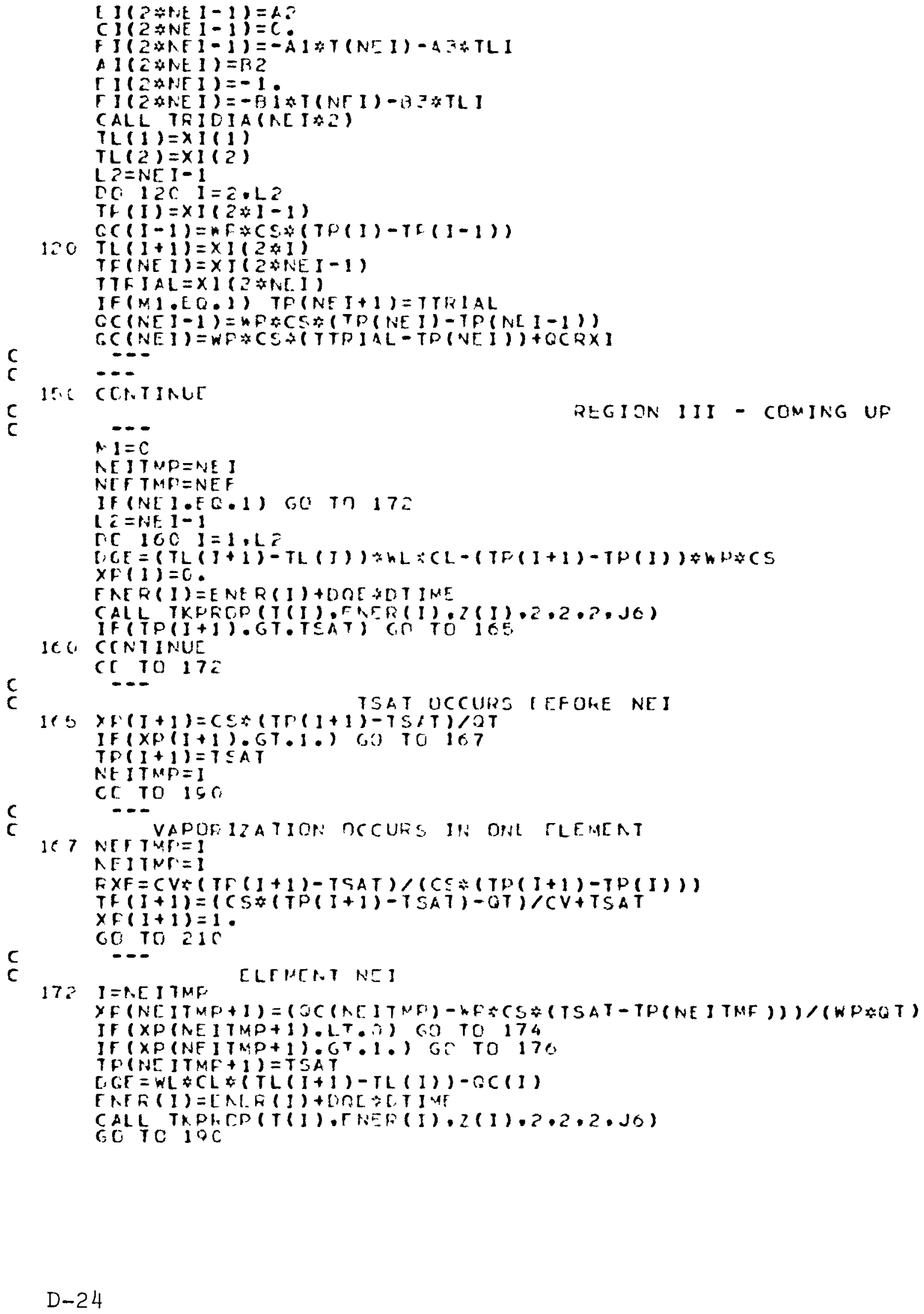




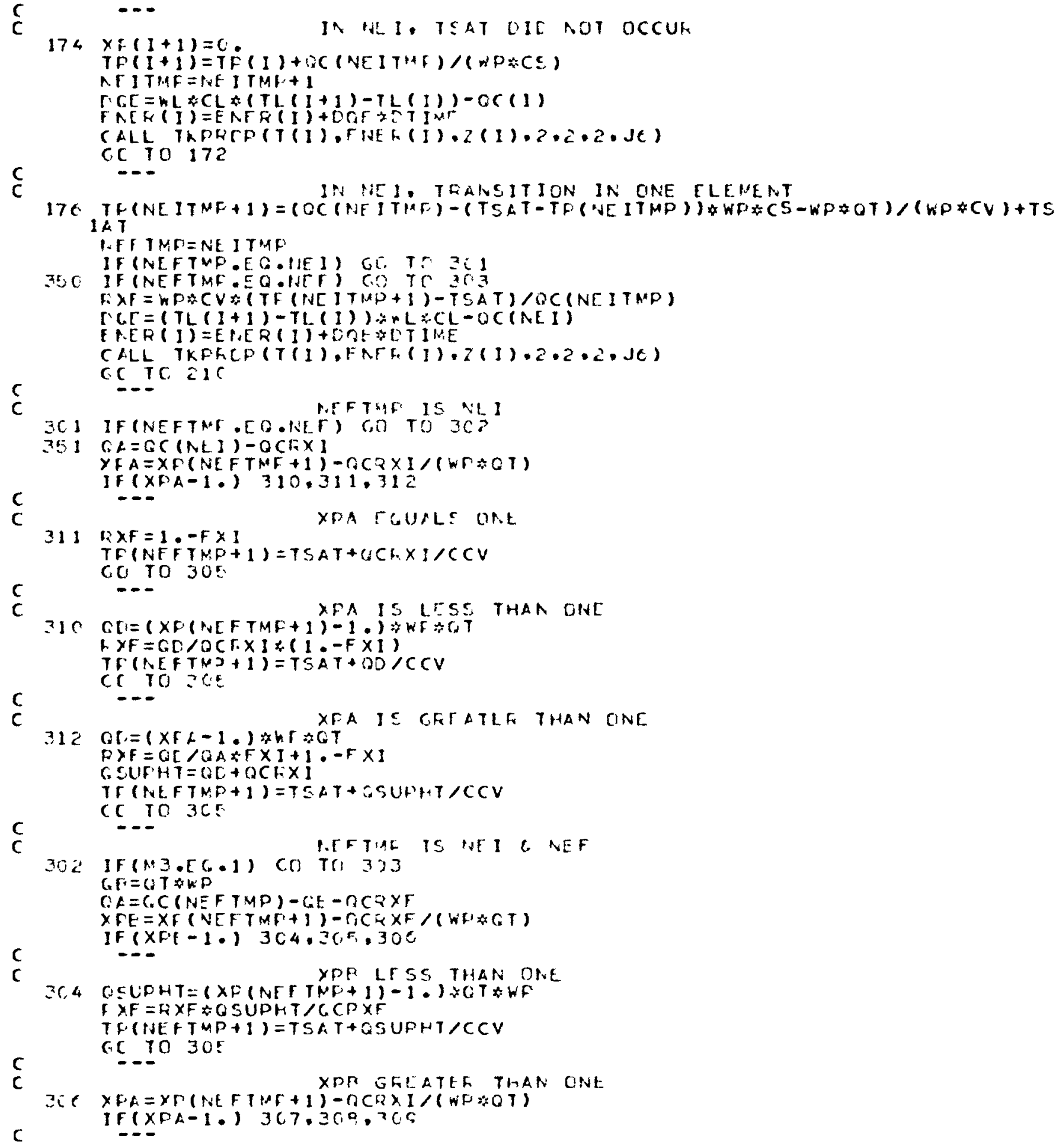




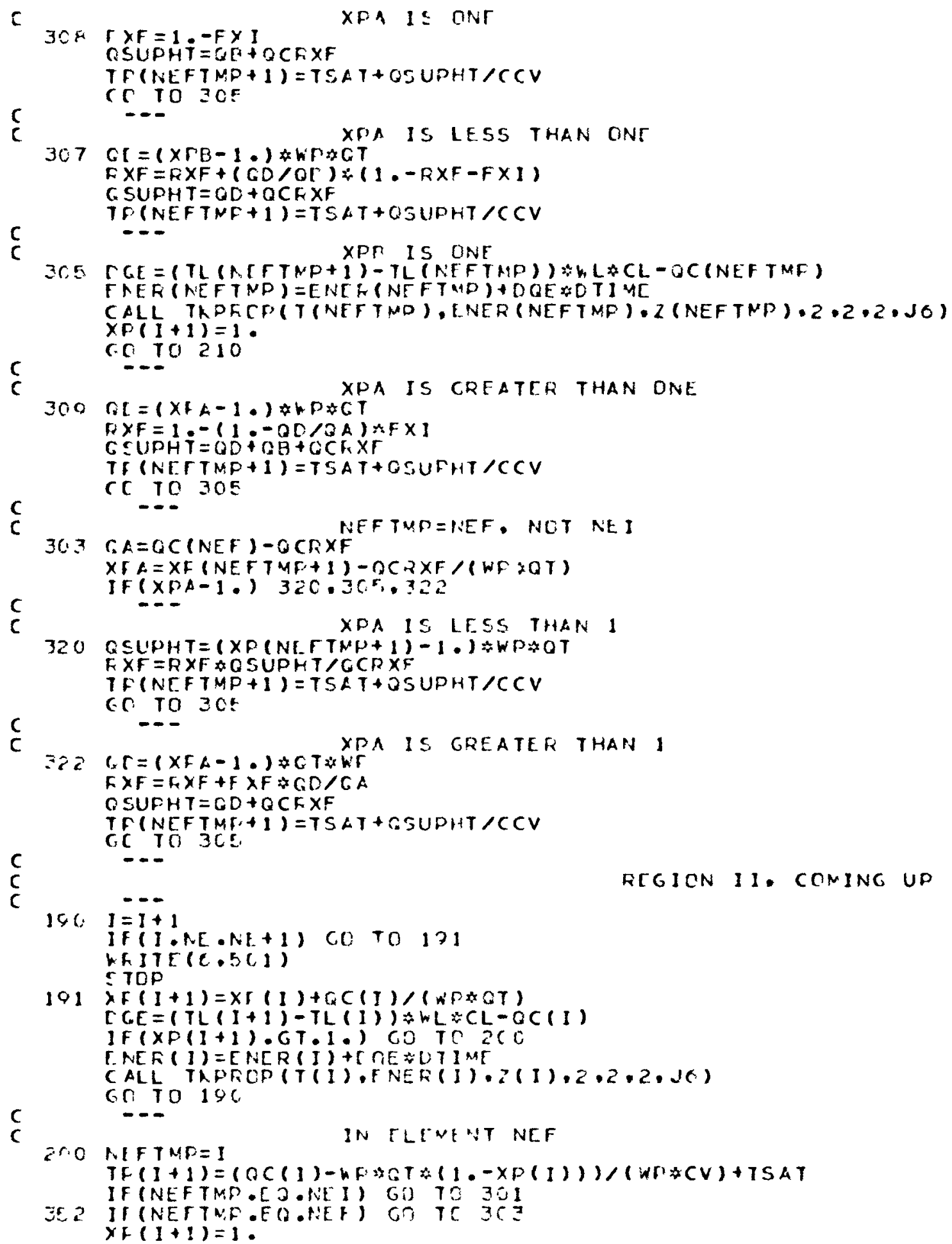




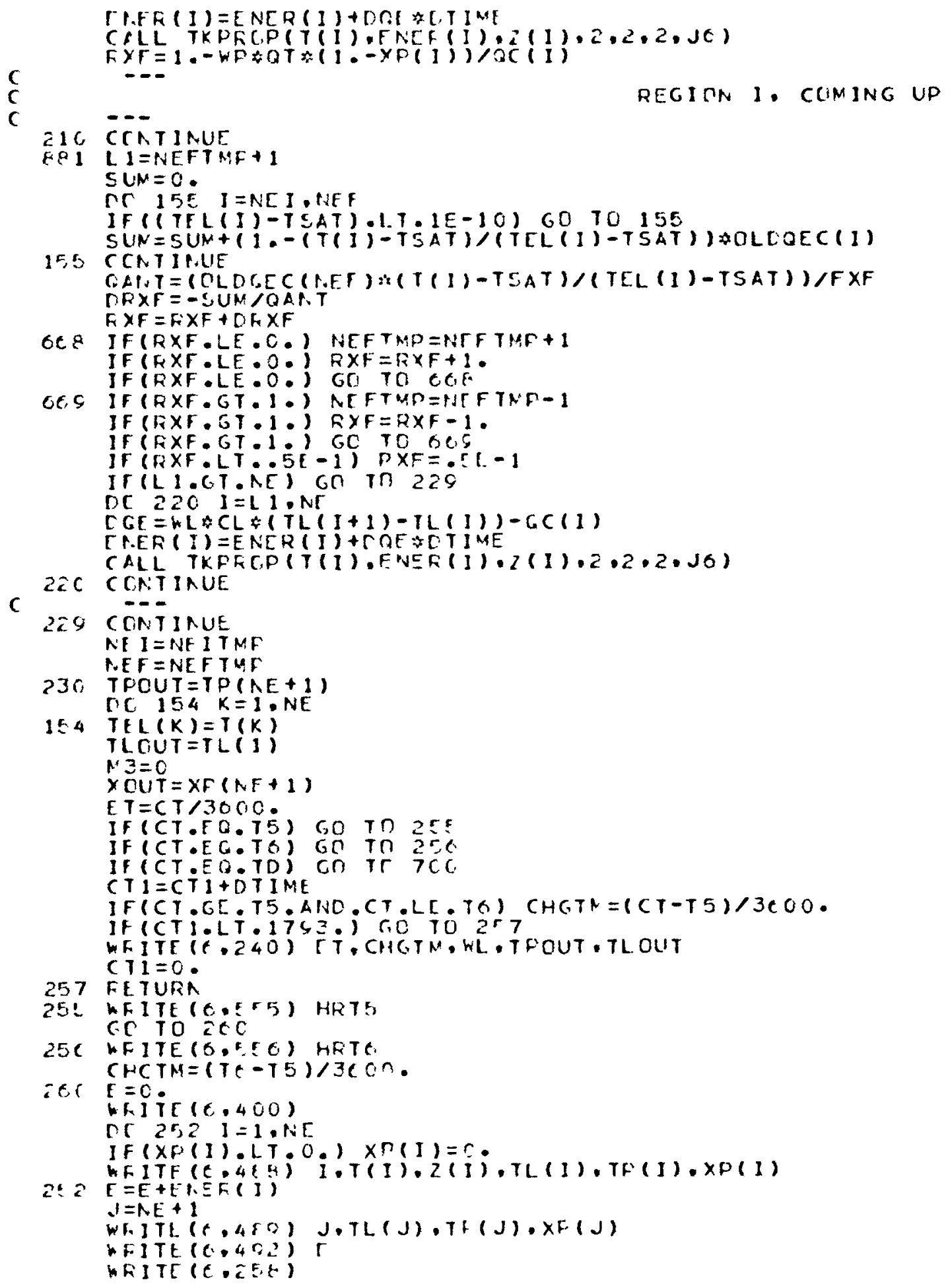




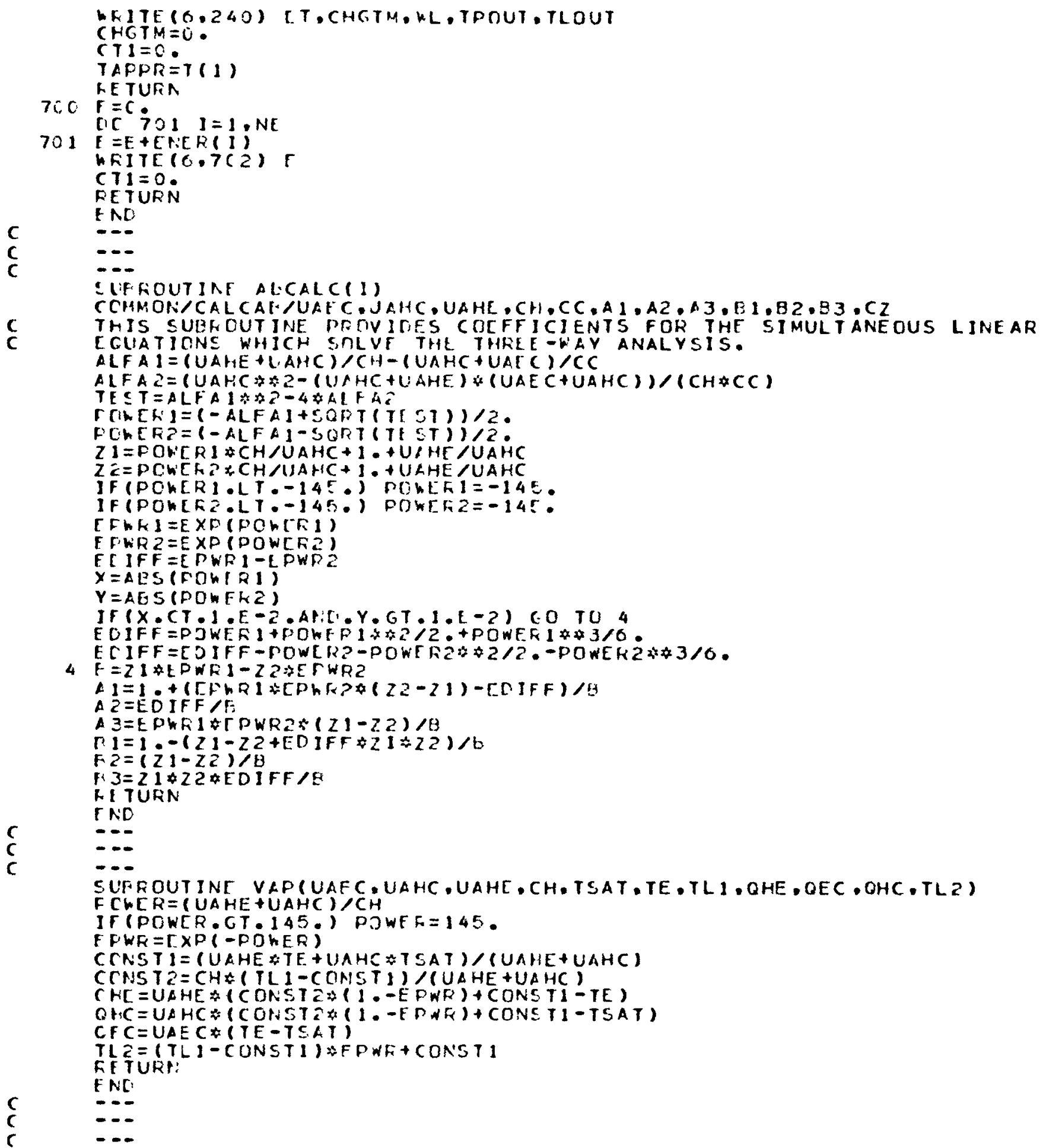




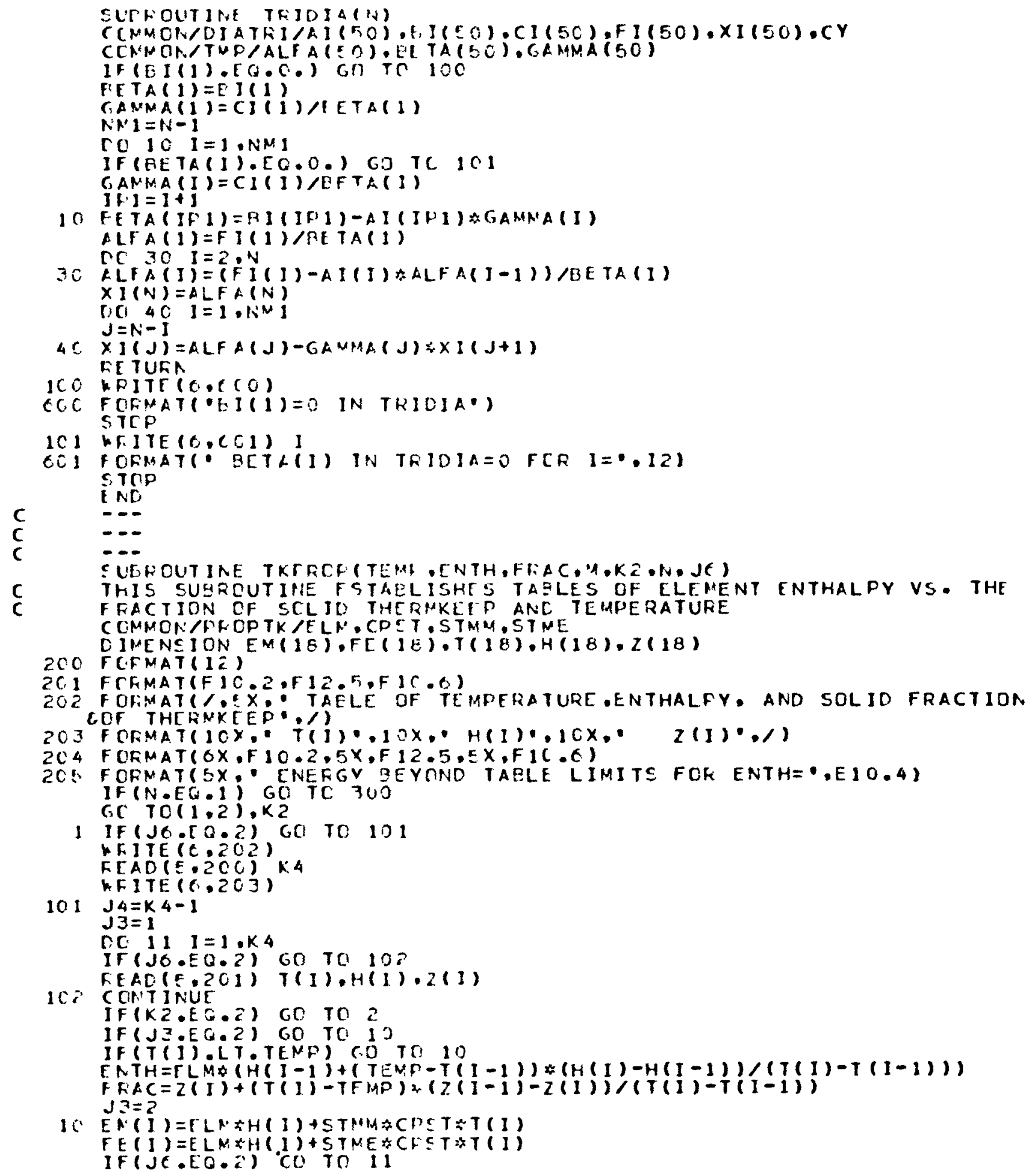

$16 E N(1)=r L N * H(1)+S T M M A C D S T \div T(1)$

$F E(1)=E L M * H(.1)+S T M E * C F S T * T(1)$

IF(JE.EQ.i) CO TO 11 


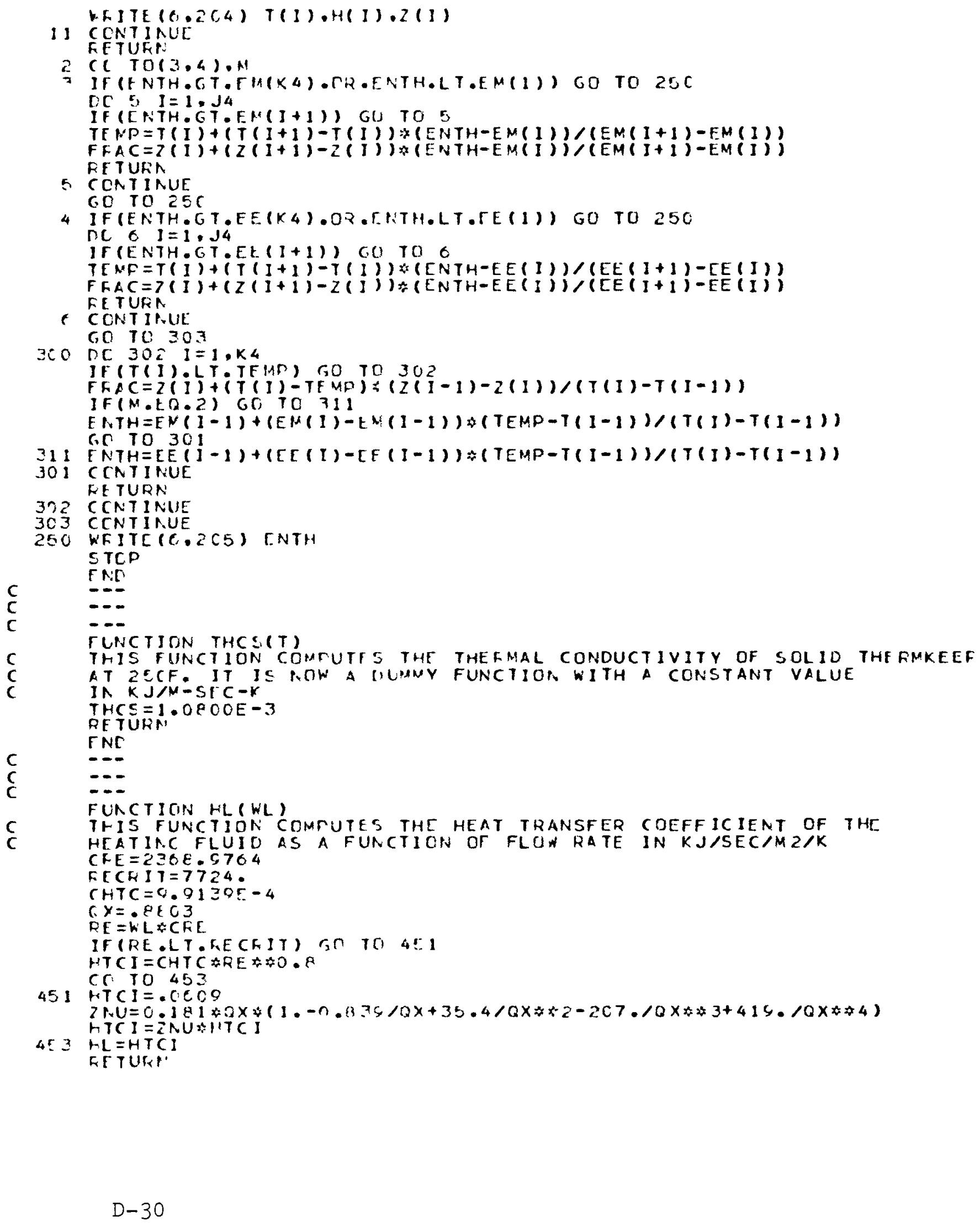




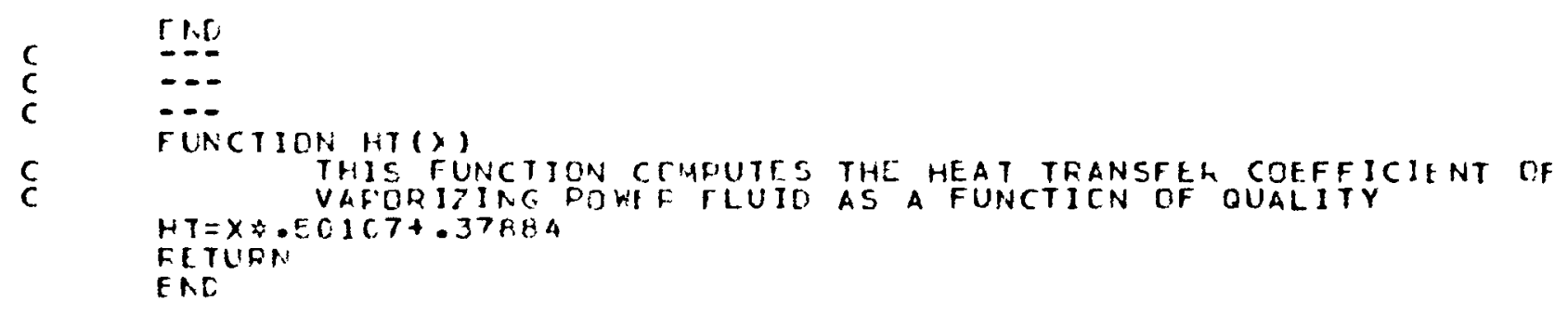





\begin{tabular}{|c|c|}
\hline$I, L$ & Integer loop counters \\
\hline $\mathrm{CL}$ & $\begin{array}{l}\text { Specific heat of transfer fluid, } \\
\mathrm{kJ} / \mathrm{kg}-{ }^{\circ} \mathrm{K}\end{array}$ \\
\hline CS & $\begin{array}{l}\text { Specific heat of subcooled power fluid, } \\
\mathrm{kJ} / \mathrm{kg}-{ }_{\mathrm{K}}\end{array}$ \\
\hline $\mathrm{CT}$ & Cycle time, seconds \\
\hline $\mathrm{CV}$ & $\begin{array}{l}\text { Specific heat of vaporized power fluid, } \\
\mathrm{kJ} / \mathrm{kg}-0_{\mathrm{K}}\end{array}$ \\
\hline $\mathrm{ET}$ & Total elapsed time, hours \\
\hline J6 & 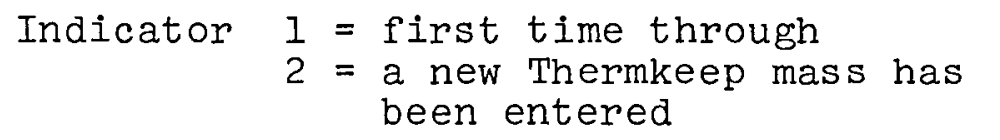 \\
\hline K5 & $\begin{array}{l}\text { The number of entries in the table of } \\
\text { solar collector output vs. time }\end{array}$ \\
\hline $\mathrm{K} 7$ & Indicator $\begin{aligned} 1 & =\text { single fluid flow } \\
2 & =\text { transition between single } \\
& \text { and two-fluid flow } \\
3= & \text { two fluid flow }\end{aligned}$ \\
\hline NC & Number of daily cycles run \\
\hline ND & Maximum number of daily cycles desired \\
\hline QC & Rate of solar collector output, $\mathrm{kW}$ \\
\hline $\mathrm{QL}(I)$ & $\begin{array}{l}\text { Rate of solar collector output at time } \\
\text { I, kW }\end{array}$ \\
\hline $\mathrm{QT}$ & $\begin{array}{l}\text { Heat of vaporization of power fluid, } \\
\mathrm{kJ} / \mathrm{kg}\end{array}$ \\
\hline $\mathrm{TD}$ & End of the daily cycle, secs \\
\hline $\mathrm{Tl}$ & Start of the daily cycle, secs \\
\hline T5 & Beginning of net charge, secs \\
\hline T6 & End of net charge, secs \\
\hline WL & Transfer fluid flow rate, $\mathrm{kg} / \mathrm{sec}$ \\
\hline WP & Power fluid flow rate, $\mathrm{kg} / \mathrm{sec}$ \\
\hline $\begin{array}{l}\mathrm{ZL}, \mathrm{ZP}, \mathrm{ZPS}, \\
\mathrm{ZPV}\end{array}$ & Coefficients used to determine time step \\
\hline $\mathrm{QCHG}$ & Heat rate storage, $\mathrm{kJ}$ \\
\hline TIME (I) & Time which matches QL(I) \\
\hline
\end{tabular}


TLIN

TPIN

TSAT

WMAX

XOUT

ASTOR

DQCHG

DQDIS

DTIME

DTIML, DTIMP

DTMAX, DTMIN

QBURN

QLOSS

TAPPK

TLOUT

TPDES

TPOUT

PCDIFF

QDEFCT

QDISCH

QEXTRA

QPOWER

QSOLAR

TALWBL
Transfer fluid inlet temperature, ${ }_{K}$

Power fluid inlet temperature, ${ }^{O_{K}}$

Power fluid saturation temperature, ${ }^{\circ} \mathrm{K}$

Maximum transfer fluid flow rate, $\mathrm{kg} / \mathrm{sec}$

Power fluid outlet quality

Amount of storage medium, $\mathrm{kg}$

Increment of storage input, $\mathrm{kJ}$

Increment of storage output, $\mathrm{kJ}$

Time step, secs

Temporary time step calculations

Maximum and minimum time steps, secs

Auxiliary heat required to maintain the desired outlet temperature, $\mathrm{kJ}$

Solar collector heat unable to be used, $\mathrm{kJ}$

Transfer fluid outlet temperature approximation at beginning of two-fluid Transfer fluid outlet temperature, ${ }_{K}$ Desired power fluid outlet temperature, $\mathrm{O}_{\mathrm{K}}$

Power fluid outlet temperature, ${ }^{\circ} \mathrm{K}$

Percent different between storage heat in and heat out

Heat required to raise the power fluid outlet temperature to the minimum allowable temperature, ${ }_{\mathrm{K}}$

Storage output, $\mathrm{kJ}$

Heat from power fluid exiting above the desired outlet temperature, $\mathrm{kJ}$

Boiler requirement, $\mathrm{kJ}$

Solar collector output, $\mathrm{kJ}$

Minimum allowable outlet temperature, ${ }^{\circ}$ 


\title{
LIST OF SYMBOLS FOR SUBROUTINE ELANAL
}

\author{
E \\ $I, J, K$ \\ $\mathrm{T}(\mathrm{I})$ \\ $Z(I)$ \\ $A I, B I, C I$ \\ The unit total energy content, $\mathrm{kJ}$ \\ Integer loop counters \\ The temperature of the Ith element, ${ }^{{ }_{K}}$ \\ The solid fraction of element I \\ Coefficients of unknowns in diagonals \\ $A, B, C$ used in subroutine TRIDIA \\ FI( I ) \\ The coefficient of the result of equation \\ I used in subroutine TRIDIA \\ $A 1, A 2, A 3$, \\ $\mathrm{B} 1, \mathrm{~B} 2, \mathrm{~B} 3$ \\ Coefficients of unknowns used to calcu- \\ late $A I, B I$, and $C I$ \\ $\mathrm{CC}$ \\ Product of the power fluid flow and \\ specific heat \\ $\mathrm{CH}$ \\ Product of the transfer fluid flow and \\ specific heat \\ $C L$ \\ Transfer fluid specific heat, $\mathrm{kJ} / \mathrm{kg}-{ }^{\circ} \mathrm{K}$ \\ CS \\ Specific heat of subcooled power fluid, \\ $\mathrm{kJ} / \mathrm{kg}-{ }^{\mathrm{O}} \mathrm{K}$ \\ $\mathrm{CT}$ \\ Cycle time, secs. \\ CV \\ Specific heat of power fluid vapor, \\ $\mathrm{kJ} / \mathrm{kg}-{ }^{\circ} \mathrm{K}$ \\ ET \\ Elapsed time, hours \\ $H L(W L)$ \\ Function which returns the transfer fluid \\ heat transfer coefficient as a function \\ of flow rate \\ $\mathrm{HT}(\mathrm{X}) \quad$ Function which returns the power fluid \\ heat transfer coefficient as a function \\ of quality \\ J6 \\ Indicator $I=$ first time through program \\ 2 = restart cycle with a new \\ storage mass \\ $\mathrm{K} I-\mathrm{K} 8$ \\ Integer loop counters \\ $\mathrm{LI}, \mathrm{L2}$ \\ Integer loop counters \\ MI \\ Condition indicator: 1 = transfer fluid \\ was calculated to increase going through \\ an element
}


M3

ND

$\mathrm{NE}, \mathrm{XNE}$

QA

QB

QD

QP

QT

TD

TL(I)

$\mathrm{TP}(\mathrm{I})$

T5

T6

WL

WP

$X I(I)$

$\mathrm{XP}(I)$

AEL

AEP
Condition indicator: $1=$ vaporization occurs completely in bottom element

Number of daily cycle in progress

Number of elements used in numerical analysis

Rate of heat flux into the power fluid stream in an element above the previous time step's point of total vaporization, $\mathrm{kW}$

Rate of heat flux into the power fluid stream in an element below the previous time step's point of incipient vaporization, $\mathrm{kW}$

Rate of heat flux into the power fluid stream in an element in the previous time step's transition region, $\mathrm{kW}$

Heat transferred to the power fluid in a whole element in the 2-phase region, $\mathrm{kJ}$

The heat of vaporization of the power fluid, $\mathrm{kJ} / \mathrm{kg}$

The cycle ending point, secs

Temperature of the transfer fluid at the lower boundary of element $I,{ }_{K}$

Temperature of the power fluid at the lower boundary of element $I,{ }_{K}$

Beginning of net charge, secs

End of net charge, secs

Transfer fluid flow rate, $\mathrm{kg} / \mathrm{sec}$

Power fluid flow rate, $\mathrm{kg} / \mathrm{sec}$

The solution to the Ith equation in TRIDIA, ${ }^{\circ}$

The quality of power fluid at the lower boundary of element I

The total contact surface between the transfer fluid and the storage medium, $\mathrm{m}^{2}$

The total contact surface area between the power fluid and the storage medium, $\mathrm{m}^{2}$ 


\begin{tabular}{|c|c|}
\hline APL & $\begin{array}{l}\text { The total contact surface area between } \\
\text { the power fluid and transfer fluid, } \mathrm{m}^{2}\end{array}$ \\
\hline $\operatorname{CCS}$ & $\begin{array}{l}\text { Product of the power fluid flow rate } \\
\text { and subcooled specific heat }\end{array}$ \\
\hline $\mathrm{CCV}$ & $\begin{array}{l}\text { Product of the power fluid flow rate } \\
\text { and superheated vapor specific heat }\end{array}$ \\
\hline CTI & $\begin{array}{l}\text { Half-hour timer for display purposes, } \\
\text { secs }\end{array}$ \\
\hline DQE & $\begin{array}{l}\text { The rate of energy change of an element } \\
\text { during a time step }\end{array}$ \\
\hline $\mathrm{EFF}$ & Heat exchange effectiveness \\
\hline ELM & $\begin{array}{l}\text { The storage medium mass in one numerical } \\
\text { element, } \mathrm{kg}\end{array}$ \\
\hline FTP & $\begin{array}{l}\text { The fraction of an element in which the } \\
\text { power fluid is in 2-phase flow }\end{array}$ \\
\hline $\mathrm{FXF}$ & $\begin{array}{l}\text { The fraction of element NEF which con- } \\
\text { tains less than quality } 1.0 \text { fluid }\end{array}$ \\
\hline FXI & The subcooled fraction of the element NEI \\
\hline $\mathrm{NEF}$ & $\begin{array}{l}\text { The element in which the power fluid } \\
\text { reaches quality } 1.0\end{array}$ \\
\hline NEI & $\begin{array}{l}\text { The element in which the power fluid be- } \\
\text { gins to vaporize }\end{array}$ \\
\hline QEC & $\begin{array}{l}\text { The heat rate from storage medium into } \\
\text { the power fluid stream }\end{array}$ \\
\hline QHC & $\begin{array}{l}\text { The heat rate from the transfer fluid } \\
\text { into the power fluid stream }\end{array}$ \\
\hline QHE & $\begin{array}{l}\text { The heat rate from the transfer fluid } \\
\text { into the storage medium }\end{array}$ \\
\hline QTP & $\begin{array}{l}\text { Heat transferred to the power fluid in } \\
\text { the two-phase region of an element, } \mathrm{kJ}\end{array}$ \\
\hline $\mathrm{RES}$ & $\begin{array}{l}\text { Resistance to heat transfer into the } \\
\text { power fluid }\end{array}$ \\
\hline $\mathrm{RXF}$ & $\begin{array}{l}\text { The fraction of element NEF in which the } \\
\text { power fluid has greater than } 1.0 \text { quality }\end{array}$ \\
\hline RXI & $\begin{array}{l}\text { The fraction of element NEI in which the } \\
\text { power fluid has greater than } 0 \text { quality }\end{array}$ \\
\hline SUM & $\begin{array}{l}\text { Anticipated heat to be transferred to } \\
\text { the superheated power fluid, } \mathrm{kJ}\end{array}$ \\
\hline $\operatorname{TEL}(I)$ & $\begin{array}{l}\text { An element temperature from the previous } \\
\text { time step }\end{array}$ \\
\hline
\end{tabular}


TLF

TII

VAP

$\mathrm{XI1}, \mathrm{XI2}, \mathrm{XI} 3$

XLE

$\mathrm{XNE}$

$\mathrm{XNT}$

XPA

XPB

$\mathrm{XP1}, \mathrm{XP2}$

AELE

AEPE

APFE

APLE

CPST

DEFF

DRXF

DSTK

ENEL

$\operatorname{ENER}(I)$
The temperature of the transfer fluid at point RXF, ${ }^{\circ} \mathrm{K}$

The temperature of the transfer fluid at point FXI,

The vaporization subroutine

Temporary points used in an iterative search for the point at which vaporization ends

The length of a tube in an element

The number of elements used for analysis

The number of tubes in each heat exchanger

The calculated power fluid quality leaving an element which contained the end of transition the previous time step

The calculated power fluid quality leaving an element which completely contained transition the previous time step

Temporary qualities used in an iterative search for the point at which vaporization ends

Contact surface area between storage medium and transfer fluid in one element, $\mathrm{m}^{2}$

Contact surface area between storage medium and power fluid in one element, $\mathrm{m}^{2}$

Inside surface area of the power fluid tube in one element, $\mathrm{m}^{2}$

Contact surface area between transfer fluid and power fluid in one element, $\mathrm{m}^{2}$

The specific heat of steel, $\mathrm{kJ} / \mathrm{kg}-{ }^{\circ} \mathrm{K}$

The effective diameter of dual heat exchanger tube

The calculated change in RXF

The diameter of solid Thermkeep buildup on a tube, $m$

The energy content of one element returned by subroutine TKPROP, $\mathrm{kJ}$

The energy of the Ith element, $\mathrm{kJ}$ 


\begin{tabular}{|c|c|}
\hline FRAC & $\begin{array}{l}\text { The solid fraction of an element re- } \\
\text { turned by subroutine TKPROP }\end{array}$ \\
\hline FSUP & $\begin{array}{l}\text { The fraction of an element which con- } \\
\text { tains superheated power fluid }\end{array}$ \\
\hline HRTS & Elapsed time at time T5, hrs \\
\hline HRT6 & Elapsed time at time $\mathrm{T} 6$, hrs \\
\hline HTCL & $\begin{array}{l}\text { Transfer fluid heat transfer coefficient, } \\
\mathrm{kW} / \mathrm{m}^{2}-\mathrm{O}_{\mathrm{K}}\end{array}$ \\
\hline HTCS & $\begin{array}{l}\text { Heat transfer coefficient of subcooled } \\
\text { power fluid, } \mathrm{kW} / \mathrm{m}^{2}-o_{\mathrm{K}}\end{array}$ \\
\hline $\mathrm{HTCV}$ & $\begin{array}{l}\text { Heat transfer coefficient of power fluid } \\
\text { vapor, } \mathrm{kW} / \mathrm{m}^{2}-{ }_{\mathrm{K}}\end{array}$ \\
\hline QANT & $\begin{array}{l}\text { Heat into the power fluid anticipated } \\
\text { for section } F \times F \text {, kJ }\end{array}$ \\
\hline RHOM & Storage medium density, $\mathrm{kg} / \mathrm{m}^{3}$ \\
\hline STME & Steel mass in an end element, $\mathrm{kg}$ \\
\hline STMM & Steel mass in a middle element, $\mathrm{kg}$ \\
\hline THCS & $\begin{array}{l}\text { Theimal conductivity of solid storage } \\
\text { material, } \mathrm{kJ} / \mathrm{m}-{ }_{\mathrm{K}}\end{array}$ \\
\hline TLIN & Inlet temperature of transfer fluid, ${ }^{\circ} \mathrm{K}$ \\
\hline TPIN & Inlet temperature of power fluid, ${ }^{\circ} \mathrm{K}$ \\
\hline TSAT & Power fluid saturation temperature, ${ }^{\circ} \mathrm{K}$ \\
\hline UAEC & $\begin{array}{l}\text { Conduction coefficient of storage med- } \\
\text { ium to power fluid, } \mathrm{kW} / \mathrm{O}_{\mathrm{K}}\end{array}$ \\
\hline UAHC & $\begin{array}{l}\text { Conduction coefficient of transfer fluid } \\
\text { to power fluid, } \mathrm{kW} / \mathrm{O}_{\mathrm{K}}\end{array}$ \\
\hline UAHE & $\begin{array}{l}\text { Conduction coefficient of transfer fluid } \\
\text { to storage medium, } k W /{ }_{K}\end{array}$ \\
\hline VSTK & $\begin{array}{l}\text { The volume of solid storage material in } \\
\text { one element, } \mathrm{m}^{3}\end{array}$ \\
\hline XNTU & $\begin{array}{l}\text { Number of heat transfer units in an ele- } \\
\text { ment }\end{array}$ \\
\hline XOUT & The power fluid outlet quality \\
\hline YNTU & $\begin{array}{l}\text { Number of heat transfer units in the two- } \\
\text { phase section of an element }\end{array}$ \\
\hline ZNTU & $\begin{array}{l}\text { Number of heat transfer units in the } \\
\text { superheated section of an element. }\end{array}$ \\
\hline
\end{tabular}


ASTOR

CHGTM

CRKTL

DTIME

ETSAT

QCRXF

QCRXI

QPART

RESTP

RSOLL ( I )

TINIT

TLOUT

TPOUT

UAECF

UAECI

UAEC2

UAHCF

UAHCI

UAHC2

UAHEF
Amount of storage material, $\mathrm{kg}$

Elapsed time from beginning of net charge, hrs.

Intermediate variable = $2 \pi \times$ XLE $\times$ XNT used in calculating solid layer resistance

Length of time step, secs

Intermediate variable used to calculate YNTU

Heat into the power fluid in section $\mathrm{RXF}, \mathrm{kJ}$

Heat into the power fluid in section RXI, kJ

Heat into the power fluid in the twophase section of an element

Resistance to heat transfer into the power fluid in the two-phase region

The solid layer resistance in element I

Unit starting temperature, ${ }^{\circ} \mathrm{K}$

Transfer fluid outlet temperature, ${ }^{\circ} \mathrm{K}$ Power fluid outlet temperature, ${ }^{O_{K}}$

Conduction coefficient of storage medium to power fluid in section $\mathrm{FXF}$, $\mathrm{kW} /{ }^{\circ} \mathrm{F}$

Conduction coefficient of storage medium to power fluid in section $\mathrm{RXI}, \mathrm{kW} /{ }^{\circ} \mathrm{K}$ Conduction coefficient of storage medium to power fluid in a purely transition element, $\mathrm{kW} /{ }^{\mathrm{O}} \mathrm{K}$

Conduction coefficient of transfer fluid to power fluid in section $\mathrm{FXF}, \mathrm{kW} / \mathrm{O}_{\mathrm{K}}$

Conduction coefficient of transfer fluid to power fluid in section $\mathrm{RXI}, \mathrm{kW} / \mathrm{O}_{\mathrm{K}}$

Conduction coefficient of transfer fluid to power fluid in a purely transition element, $\mathrm{kW} /{ }^{\mathrm{O}} \mathrm{K}$

Conduction coefficient of transfer fluid to storage medium in section $\mathrm{FXF}, \mathrm{kW} /{ }^{\mathrm{O}} \mathrm{K}$ 
UAHEI

UAHE2

XLEFF

XPXI3

CUAHCS

CUAHCT

CUAHCV

NEFTMP

NEITMP

OLDQEC (I)

QSUPHT

RESSUP

TTRIAL

XNTUSP
Conduction coefficient of transfer fluid to storage medium in section $R X I, k W /{ }^{\circ}$

Conduction coefficient of transfer fluid to storage medium in a purely transition element

Tube length, m

Intermediate variable used in an iterative search for the vaporization ending point

Conduction coefficient of transfer fluid to power fluid in partial elements for subcooled, transition, and vapor phases, $\mathrm{kW} /{ }^{\circ} \mathrm{K}$

Element where end of transition is temporarily located

Element where the beginning of transition is temporarily located

Heat transferred from storage to power fluid in the Ith element during the previous time step

Heat into the power fluid in the vapor region of an element

Resistance to heat transfer into the power fluid in the superheated region

A calculated power fluid temperature at the beginning of two-phase flow, $\mathrm{O}_{\mathrm{K}}$

Number of heat transfer units in the superheated fraction of an element 

A P P E N D I X $\quad$ E

SINGLE HEAT EXCHANGER COMPUTER CODE

$E-1$ 

MI IN PRCGRAM TESPF

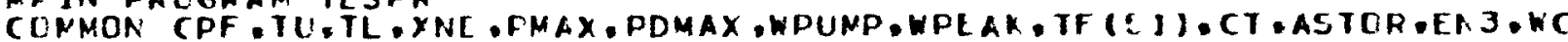
C.OL

DIMENSICN OOUT(25). TIN(25)

3CC FLPMATII3.OF 1C.1)

301 FURMATI19X. TABLE CI SOLAR COLLECTOF DUTPUT VE. IINF 1

3CË FCRMAT (CAX: FATE IN KW: OX. TIME IN SECONLS:)

303 FCFMAT(FIO.4.F10.1)

304 FCRMAT (2F10.4.EF 10.1 .F 10.0.F10.2.F JC.3)

30E FI FMATI5X. FLUID SPFCIFIC HEAT. KJ/KG/K :IIC.4)

$30 C$ FCFNAT(5X:- THERMKEE SPFC HEAT:KJ/KG/K : F 10.4 )

307 FERMATI5X. TLRBINE INLET TEMP. K

30 FCRMATIEX: TLPBINE CUTLET TEMP...K K :F10.1)

3CG FIRMATIEX. NC. OF ELENENTS FOG ANALYSIS -FIO.C)

310 FCFMAT(SX: AAT. OF STORAGE MATL.. KG OF IC.E)

311 FCRMATI5X: MAX: PUNE FLOL.KG/SEC. :F10.Z)

312 FCRMATIEX. MEX TINF INCREMENT. SFC.

313 FLRMAIISX: BIILEF DENAND. KW

314 FIRMAT (IHI.EX. MAIN FROGRAM DATA. ./1)

315 FCEMAT (ZEX F IC.4.9X,F10.1)

31 FCRMATIEX: NC: OF CA ILY CYCLES

317 F (PNATIIHI.5\%. HEAT CF FUSION THEKNAL STOFAGE GRALYSIS*

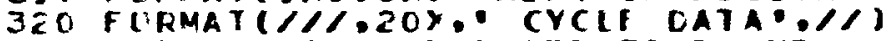

ZË FLFMAT (1OX. ELAFSEC TINE. HR

322 FCRNATIIOX: CYCLE TINE. SEC

FLRNATIICX. LOST COLLECTOR HEAT. KJ

$\$ 24$ FCRMATIIOX: CCLLECTCK OUTPUT.KJ

32 FIRMATIIOX: SIDFACE CUTPUT. KJ

326 FI NNATIIOX. STORAGE JNPUT. KJ

327 FLPMATI 1OX: AUXILIAFY INFUT.K.

32 FCFMAT (10X. ECILEF FEGUIREMENT * KJ

329 FCFNATIIOX: TEAK STCFAGE FLOW. KG/S

330 FCFMAT (10X. IEAK FRESS DFOP. KCF/SM

334 FERNATL10X. TEAK PFESS DKOP. PSI

331 FCFMATIIOX: TEAK PLIF PCLER: KE

332 FIFMATIIOX: - TOTAL FUNP WCRK. KK-HR

333 F[RMAT (3F 10.2.F 1C.3.13)

33E F[FMAT(10X. : RUMEFF CF TINE INCAEMENTS*.110.1/\%)

उ3E FCFMATIEX.: MIN. TINF INCFEMENT. STC. :FIC.Z)

337 FCPMAT(EX: ICLE TIME INCREMENT: SFC:

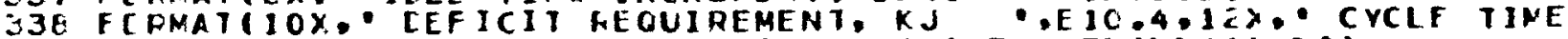
CFLCW

340 F[FMAT (F 10.0$)$

FLAD (5.300) KC.T1.72.T3.T4.T5.TC

FE AD (5.304) CFF.CTK.TU.TL.XNE:ASTOR.MNAX

RE $\angle E(5.333)$ CTNAX.DTA IN.DTIDLE. GE.ND

$J f=1$

ZEC CLNTINUE

IF ( $36 . E G .2)$ GC TC $37 C$

WF JTE $(6.317)$

WF ITE (6.301)

WF ITE (6.302)

DE I $1=1 . K 5$

READ(5.303) CCUT(I).7IN(1)

370 WFITE(6.314) 


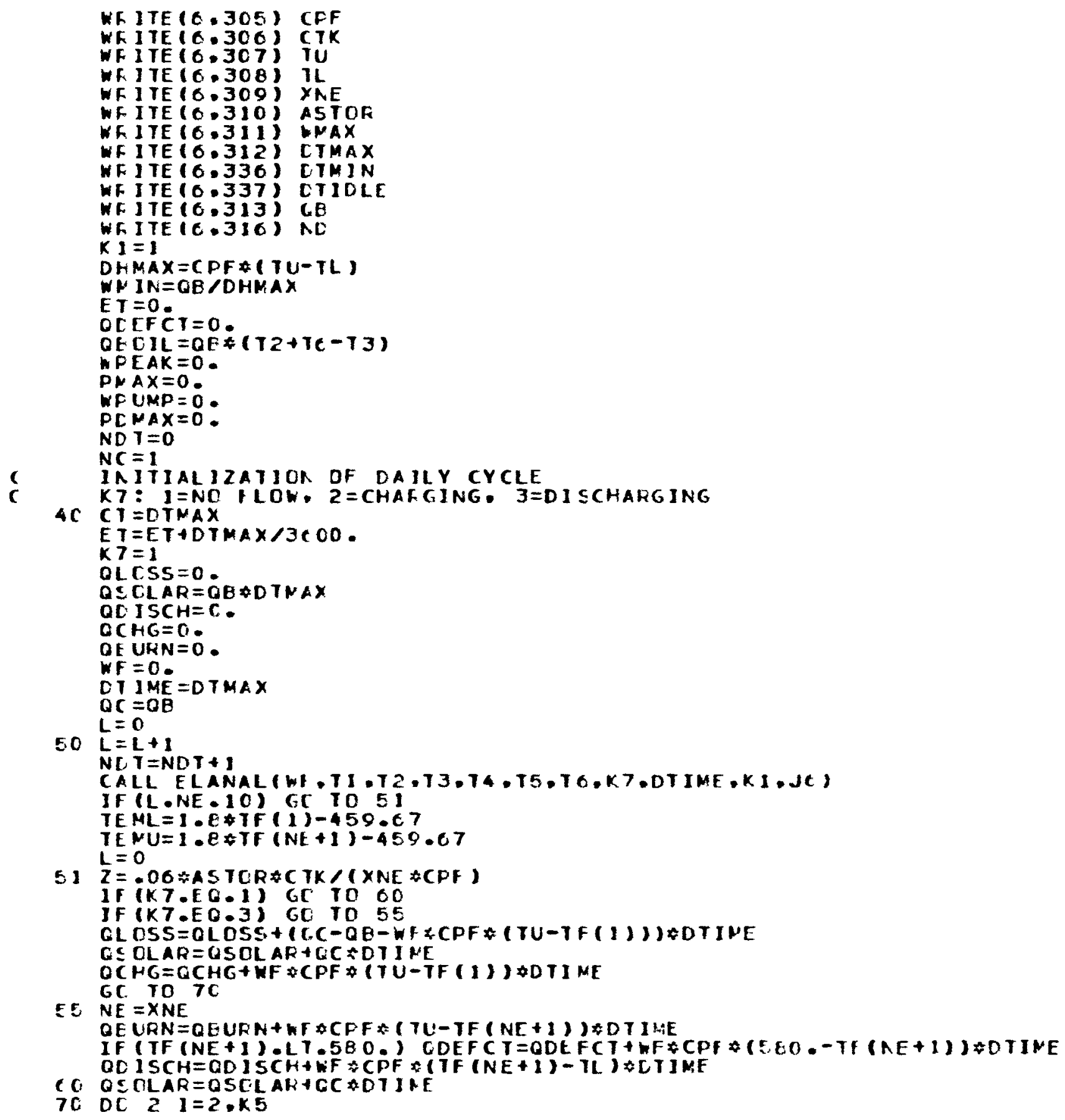




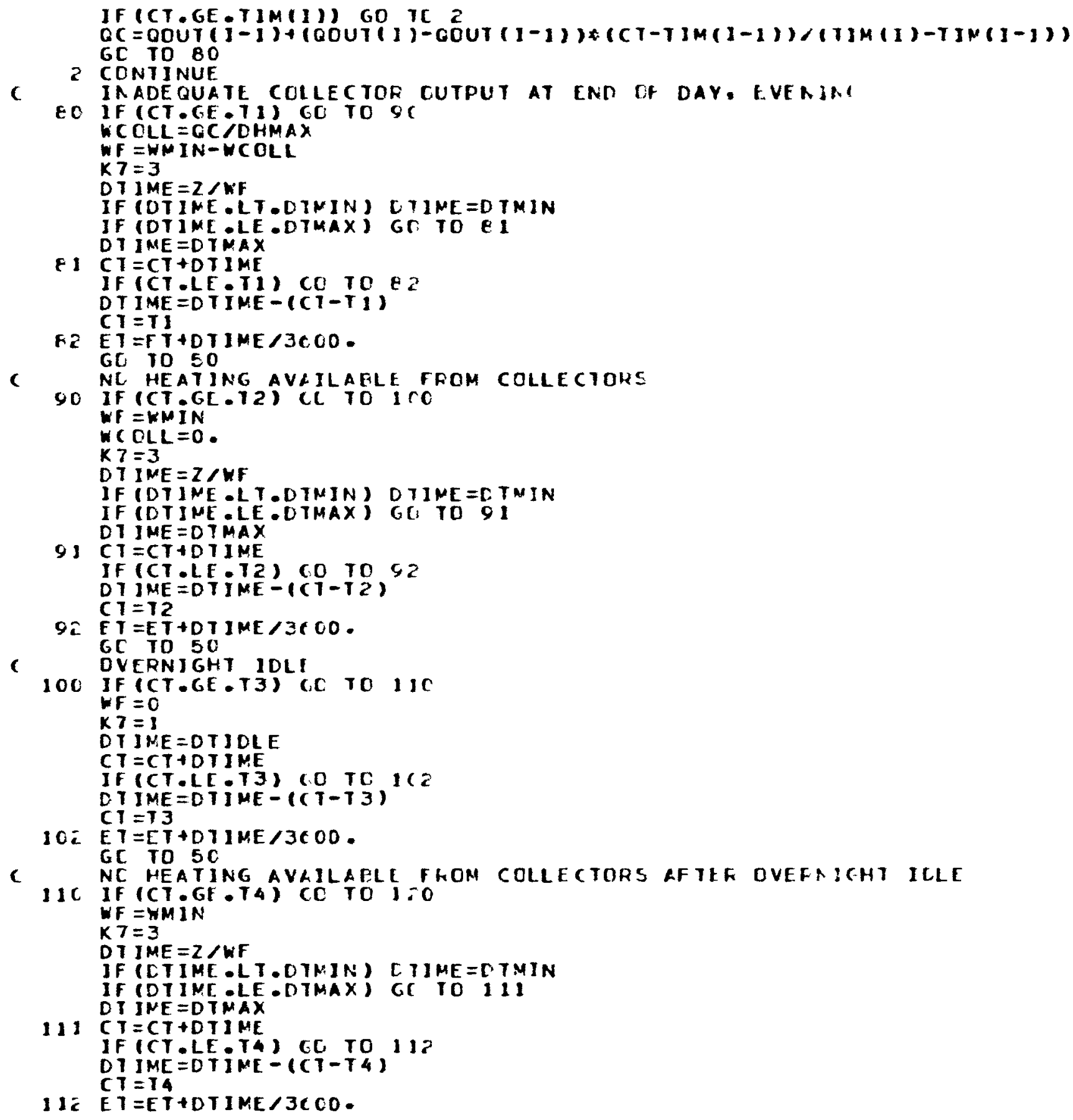

IIC ET $=E T+D T I M E / 3 C O O$. 
MAOEOUATE COLLECTGE CUTPUT AT EFGINNING CF TAY NOFNING,

12 C IF (CT.GE.T5) C.C TC $1 \equiv 0$

WCCLL $=O C / D H M A X$

$W F=W M I N-W C D L L$

$K 7=3$

D 1 IME $=2 / W F$

IF (CTIME -LT DDTNIN) DTIME = OTNIN

IF ICTIME:LE:CTMAX) GC TO IZI

DT IME $=D T M A X$

12) $C T=C T+D T I M E$

IF (CT.LE.T5) GO TO 1:2

DT IME =DTIREE $-($ T T -75$)$

$C 1=T E$

$122 E T=E T+D T I M E / 3 C 00$.

Gr 1050

C

EXESS SULAR HEATING AVAILAGLE FOR STCRAGL

$13 C$ IF (CT.GE,$T O$ ) GC TU 140

IF (CT.GT.T5) CO TC 131

$H=0$

$K 7=1$

DT INE $=$ OTMAX

GC TO 132

13) $W F=(O C-O B) /(C F F(T U-T F(1)))$

WCLL $=W F+W M I N$

IF (WCCLLOGT WNAX) WF =WMAX-WNIN

IF (HCOLL.GT G MAX) WCCLL WMAX

$K 7=2$

DT $1 M E=2 / K F$

IF (OTIME.LT.OTMIN) OTIME = TTMIN

IF (OTJME $-L E \subseteq D T M A X)$ GC TC 132

13: CTIME $=$ DTMAX

IF (CT-LE.T6) CD 10 133

DT IME $=0$ TINE-(CT-TC)

$\mathrm{CT}=1 \mathrm{O}$

$133 E T=E T+D T I V E / 3600$.

GC 7050

C

CUNULATIVE INFOFMATICN AND MAIN FRTGRAM EAD

140 TIE $(0.320)$

WF ITE $(0.321)$ t T

WF ITE $(0.323)$ CLDSS

WF ITE 16.324$)$ CSOLAR

WF 17E(6.325) COISCH

WF ITE (6.326) CCHG

WF ITE (6.327) CEUFN

Wh ITE $(0.338)$ CDEFCT

WF ITE $(0.328)$ CBOIL

WF ITE $(6.329)$ HPEAK

WF ITE (6.330) FDMAX

PSIMAX =PONAX*.00142-

WRITE $(6.334)$ FSINAX

WR ITE $(6.331)$ FMAX

WF ITE $(6.332)$ WFUMF

WF ITE (6:335) P.TT

$N C=N C+1$

OC ]FF I $=0 . Q^{*} * N^{3}$

OD IFF $2=O C H G-C L I S C H$ 


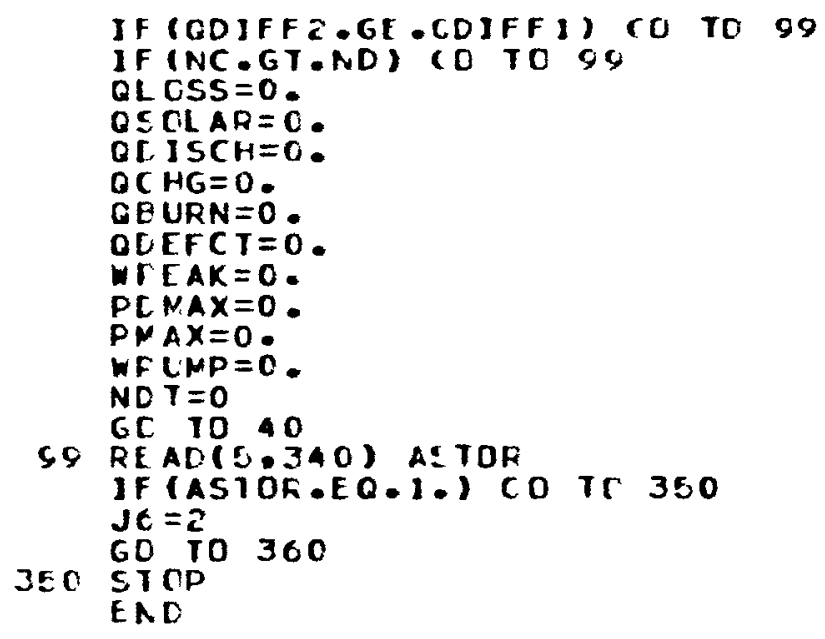


SLEROUT INE ELANAL (WF.T1.T2.T3.T4.TE.T6.K7.LTINE.K1.J6)

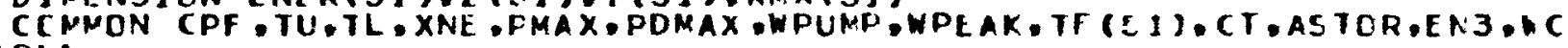
COLL

CCMMON/PROFTK/ELM.CPST.STMM.STME

I $(J 6 . E O .2) \mathrm{CC}$ TO $3 R \mathrm{C}$

$G 0$ TO $(1: 2) \cdot K]$

FIFST TIME THFCUGH - INITIALIZL AND FEAO CATA

1 NE $=X N E$

CT $1=0$.

RE AD (E.101) OC.DI.XLT.XNT.TINS.COO.OIO

RE AD (5.102) TEND. TSHTLL.DHEL

RE AD (5.103) RHOST.FHCF RHOM.RHOINS

REAC(B.104) THCINS.THCST.IHCF.PFF.VISCF.CPST

READ (5.105) TAMB.TSHF.TINIT

READ (5.70C) PNATS.PF CSA.PF SSU.PFSTA.PFSTW.PISEA.FISEW

READ(5.701) GATOPS.GASIDE. GABOTS.PNVW.PYVE FFVV IFFV

READ (5.702) CFMTW.PFTH.DFTA.PTK .PINS.ASFECT

360 CCNTINUE

IF (KI.TO.2) GC TO 2

C

GE OMFTRICAL CALCULATIONS

VTUEE S = -7854*XLT*XNT*CO*2

VCLTK $=$ ASTOR/RI.OM

VTOT $=$ VOLTK+VTLEES

ATTEMPT SFECIFIED ASIFCT RATIO

$J E=1$

DSHELL $=($ Y TOT $* 1.27324 / A S T E C T) * *(1 . / 3$.

HS HELL =DSHELL $* A S P E C T$

If (HSHELL LE.XLT) GO TO 3

HSHELL $=X L T$

DSHELL $=2$ * SOFT(VTOT/(3.1416*HSHELL))

$J E=2$

c

3 DSHR=DSHELL+2 * T INS+ $2 *$ TSHELL

WE ICHT CALCULATIONS

HGTTOT $=$ HSHELL+2. $+11 N S+2 *$ TEND

WTENDS $=1.570 E \div$ TEND*FH.CST $*$ D SHELL $* 2$

WT SHEL $=3$. $1410 \times 0$ SHELL TSHELL \$HSHCLL $\$$ RHCST

WTVES = TSHEL THTENDS

WTTUEE $=: 7 E 54 * 1 D D * 2-[1 * 2) *$ RHOST*XLT*XNT

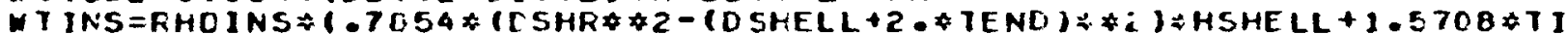

\&NI USHF $\$ 21$

CCST CALCULATIONS USIH.G SIX-TENTHS FACTOR

A TUEES $=3.141 C * X L T * X A 1 * 0 C$

PNTW=CPNT $\angle D C$

C TUEE $=W T$ TUBE TPMTW

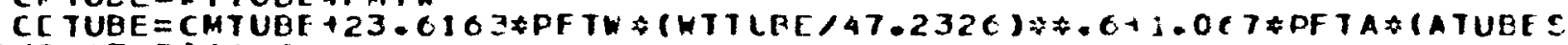

$*(2.1343) * 60$

COSTOR=ASTOR + T TK

CE INS =WIINS\#FINS

ACIDES $=3.1410$ ODSHF HCTTOT

TSIDE $=$ ASIDES $\approx G A S$ IDL $\approx$ RHOST

CNSIDE = WTSIDL XFA $1 S$

CCSIDE $=$ CMSIDE+23.92*FFSSW*(WTSIDE/47.E4)*\#.6+2.:*6F:SA*(ASICES/5.) $* * \cdot C$ 
AEOTS $=-7 E E 4 \% C E H R * 2$.

LTEOTS=AEOTSNCABOTS FHOST

CNRCTS $=$ TEOTS*FMATS

COBOTS=CMEOTS+E. I7*FFEBW*(WTEOTS/1C.34)**C+.3C:S5*FFSEA * IAEOTS/.C $+119) * 0$

ATCPS = AEDTS

WT TOPS = ATOPS $*$ GATOPS FFMOST

CMTOPS = TTOPS TPMATS

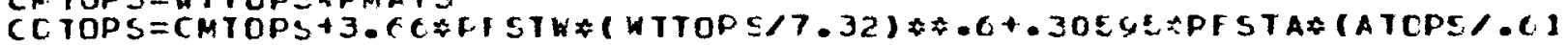
$* 15) \neq * 0$

CNSHRD $=$ CMSICE +CMBOTS +CMTOF 5

CC SHRD = CDS IDE + CDBDTS +CDTOPS

CNVESS=WTSHEL $A F M V W+W T F N O S$ AMVE

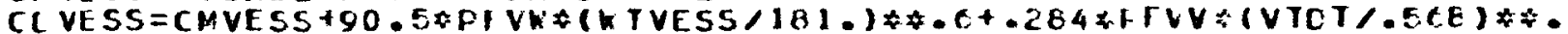
$\forall \mathrm{C}$

CNTOT $=$ CMTUEE + CNSHRD+CNVESS + CDINS+COSTOR

CCTDT = COTUEE + COSHFC + CCVESS + COINS+COSTCR

CCFTES $=$ COTOT-CNTOT

T THRD $=$ TSIDE THTTOPS HTECTS

WT TOT = TVESS + TTUPE + WTINS + ASTOF +WTSHRD

RESISTANCE CALCULATICNS - LATEFAL

FL ARE A $=0.7854 * N T * 1,1 * 2$

RE CFIT $=2100 *(1 .+12 * *(D) / L+H E) * C .5)$

CFE =DI/ (FLAREA I ISCF)

CH TC $=0.022 *$ THCF* (FRF $*-4) / D I$

A 1 UHE $1=3.1416 * X N T \neq 01 \leqslant X[T / X \mathrm{NE}$

CFKTL $=C-2 E 3 \times \times L T \circ \times N T / X I V E$

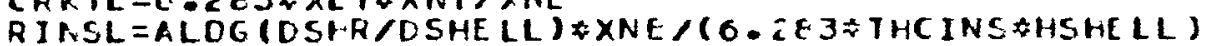

RE SISTANCE CALCULATICNS - AXIAL

RT UBEA $=X L T /(.7854$ THCST*XNT*XNE*(DO*2-DI**2) $)$

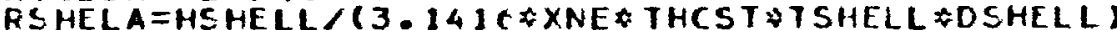

CKKMA $=$ HSHELL/1.7854*XNE*(DSHELL *2-XNT*0C*\$2))

RF SISTANCE CALCULATIINS - END

RT UBEE $=11 N 5 /(.78 E 4 * T / C S T *(000 * 2-010 * 2))$

RINSE $=11 N S /(.7 E 54 * T H(J N S *(D S H R * 2-X N T * D D * 2) 1)$

RAXE $=1 . /(1 . / K T U B E E+1 . / R I N G E)$

MASS CALCULATIONS

ELM=ASTOR XNE

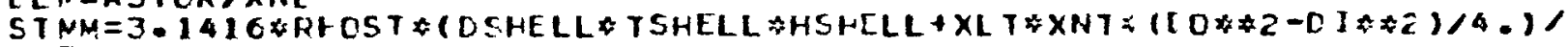
$1 \times \cap E$

STME $=5 T M M+.7 E E 4 * R H C S T * T E N D * 10 S H E L L * 2-X N T * 01 * 21$

ICI FCRMATIZF10.E.F10.2.F10.0.3F10.5)

102 FCRNAT(3F10.6)

103 F(RMATI 4FIO.Z)

104 FCRMAT ( 3F 10.9.F 10.5.110.9.F10.5)

jOS F[RMAT (3FIO.2)

3 FE FRMAT(1\%,12Y. SUPFCUTINE FLAMAL CATA.:1)

107 F[FNAT (EX. SHRQUD DJAMETER. M.

10E FETINATIEX: VESSTL CIAMETER:M: -.F10.4)

109 FCRMAT (SX."TUEE D.D..M. $. F 10 . E)$

$11 \mathrm{C}$ FGFMAT(SX:- TUBE I.D.:M.,$F 10.6)$

11 FLRMAT(SX. VESSEL HIJGHT. M..$F 10.4)$

11 FIFNATISX: TLBE LENGTH...,$F 10.2)$

113 FCRMAT (5X: NC. DF TUEES : 10.0$)$

114 FLRMAT (5X. IMSULATICA THICKNESS." " F10.5)

115 FORNAT (5X: VESSEL HAL THICKNESS. M.: If 10.6)

1 IL FCFMAT (5X. VESSEL ENC THICKNESS.N. *F10.l)

117 FCRNAT(SX."THERNKEEF DENS . KG/CU.M.*F1C.2) 


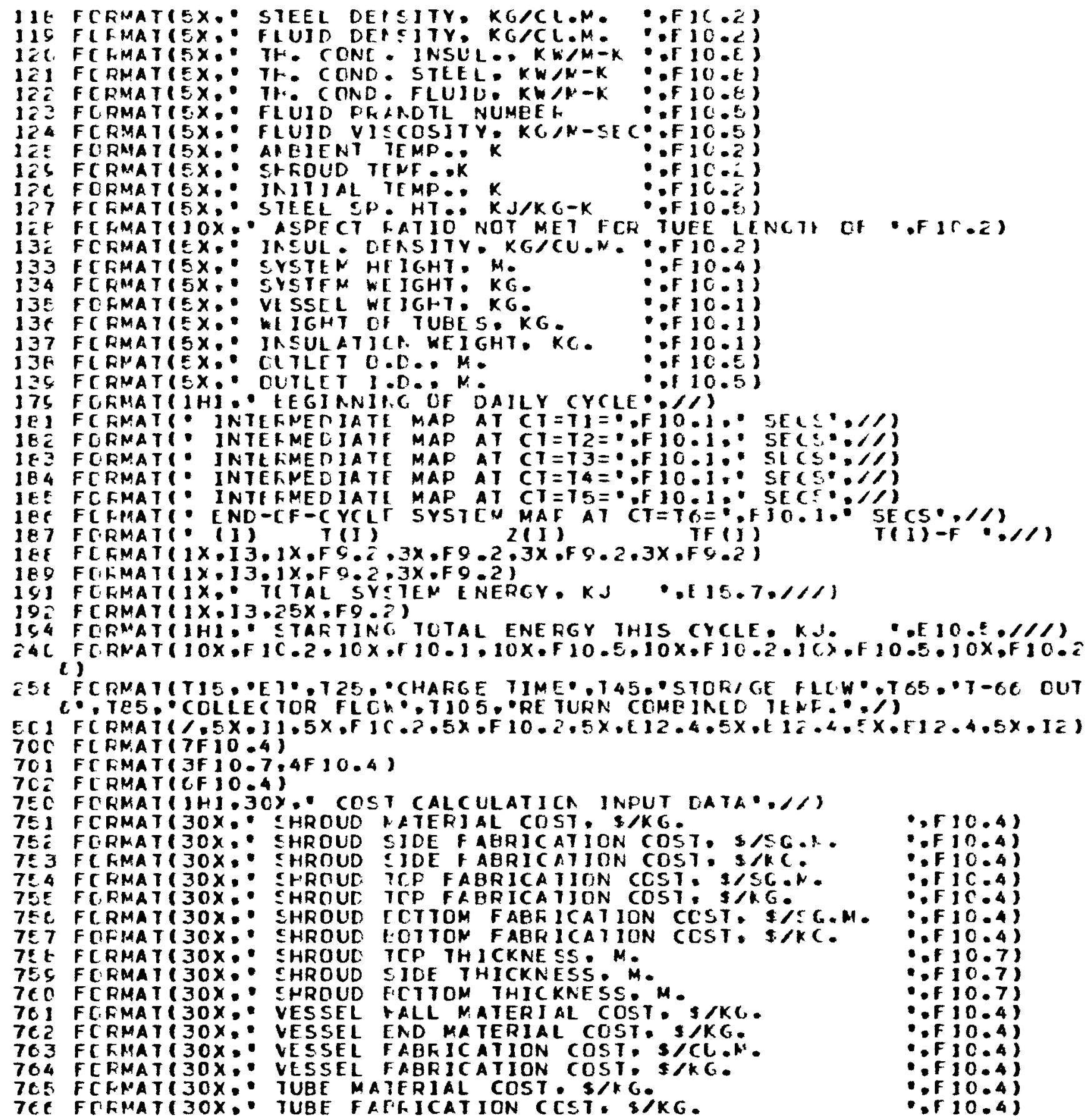




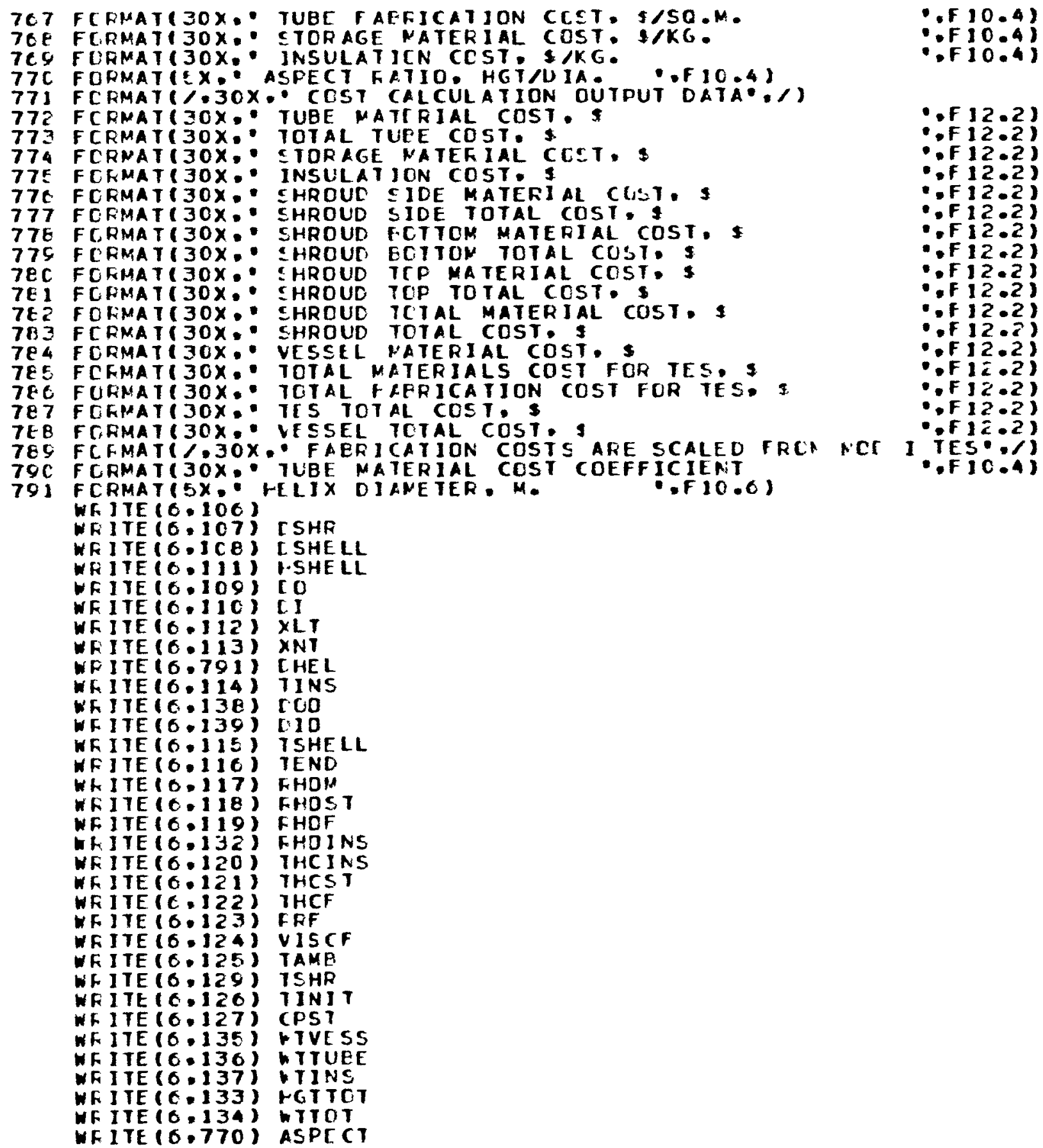

-.F10.4)

- F10.4)

- F 10.41

- F 12.21

- F 12.2)

- F 12.2

- F 12.2\}

- F 12.21

- F 12.23

$\because F(2.2)$

- F 12.21

- F 12.2J

$\because F 12.21$

- $F 12.21$

- $F 12.8$ )

- F12.2)

$\because F 12.2\}$

$\because F 12.2)$

- F 12.2 )

$\because F i(-2)$

1 TES: $: 1$ $\because$. 10.43 
IF (J5 .EC.1) GC TO 4

WF ITE (6.12E) XLT

4 W ITE $(0,750)$

WF ITE $(6.751)$ FMATS

WF ITE $(6.752)$ FFSSA

WFITE $(6.753)$ FFSSW

WFITE $(0.754)$ FFSTA

WFITE (6.755) FF5TH

WF ITE (6.7EC) TFSEA

WF 1TE (6.757) PFSBW

WF ITE $(6.758)$ GATOPS

WF ITE (0.759) CASIDE

WFITE $(6.760)$ GABCTS

WFITE (0.761) FMVH

WF ITE $(0.762)$ FMVE

WK ITE (6.703) FFV

WFITE 10.704$)$ FFVW

WF ITE (6:790) CPMTH

W ITE $(6.765)$ FMTK

WFITE $(0.700)$ FFTW

WF TE $(0.767)$, FTA

WF ITE (6.76E) FTK

WFITE $(0.769)$ FINS

UKITE $(6.789)$

$\forall$ TTE $(6.771)$

H ITE 10.772$)$ CMTUEE

WFITE (6.775 COTUEE

WK ITE (6.774) CCSTOR

WF ITE $(6.775)$ CDINS

WFITE (6:776) CMSIDE

W. ITE (0.777) CDS1DE

WKITE $(0.778)$ CMBCTS

WGITE $(0.779)$ COBDTS

WRITE (0.7EO) CMTCFS

WPITE $(6.781)$ COTDPS

HFITE (6.782) CMSHFD

WFITE $(6.783)$ COSHFL

WFITE (6.7EA) (MVESS

WFITE $(6.786)$ COVESS

WFITE (B.78E) CMTOT

WFITE (0.786) COFTES

$c$ WFITE $(0.787)$ COTOT

IAITIALIZE TAELE DF FLEMENT ENEFGICS AT TINIT

CALL TKPRDPITINIT.ENEL.FRAC.1.1.J6)

$E=0$.

DC $5 \quad 1=1 . N E$

E T I I I TINIT

$Z(1)=F R A C$

$E=E+E N E R$ (I)

F CCNTINUE

WFITE $(6.194)$ E

$K 1=2$

C

DECIDE FLOW OF NO-FLCW

C IF (KT.NE.1) GC TO IS

OF $L=0$

GC TO 20

IE RE $=\forall F \approx C R E$

INSIDE HEAT TEANSFER COEFFICIENT ART FIEISIANCE 


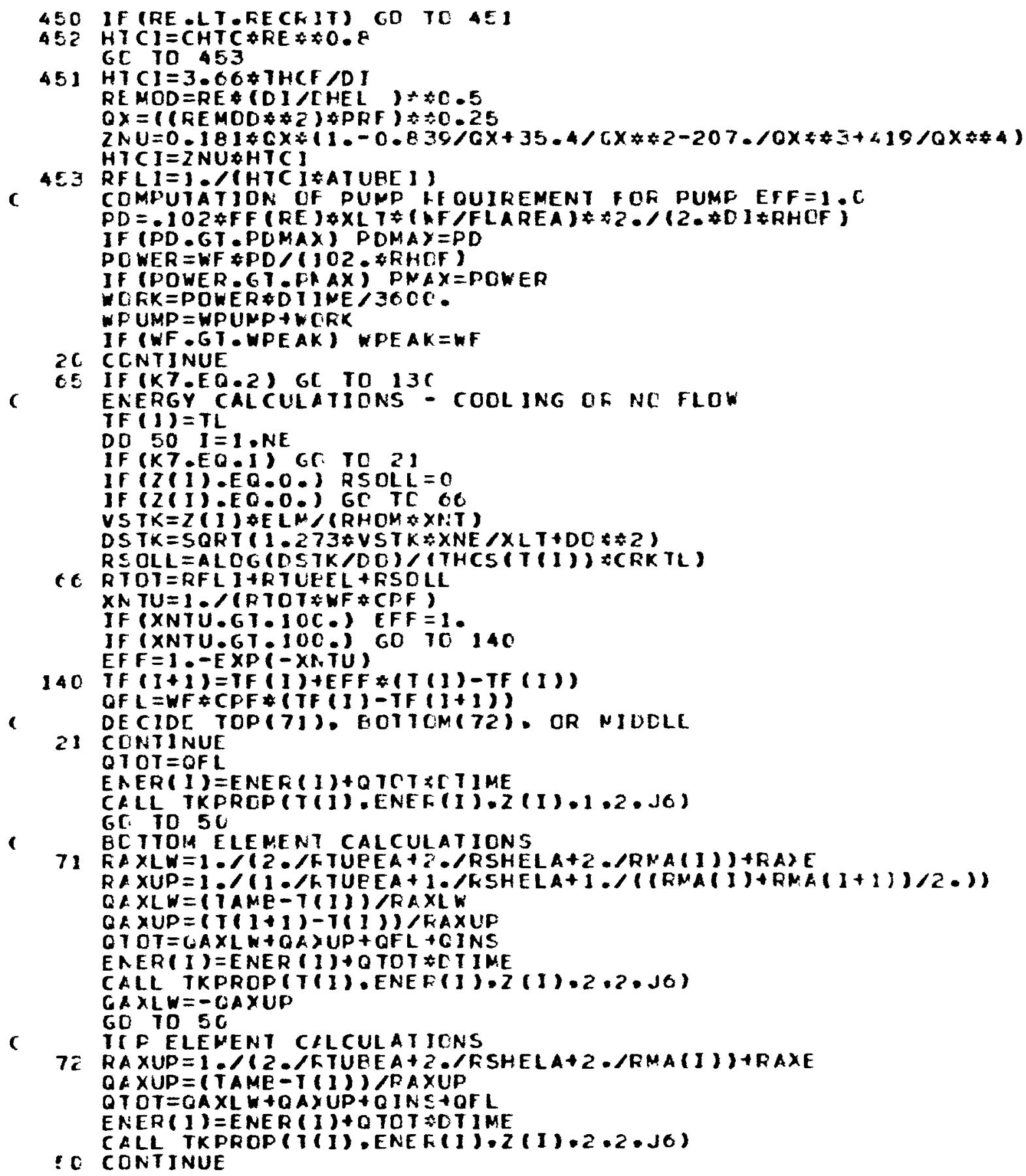

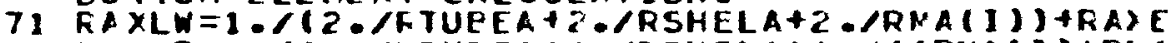

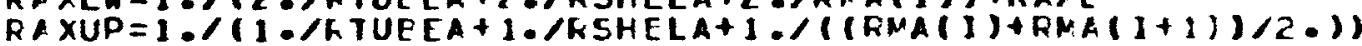
QAXLW =(1AME-T(I) IRAXLW QAXUP $=(T(1+1)-T(1) / F A X U F$ O1OT=GAXLW +OAXUP + OFL +CINS ERER(I)=ENER(1)+OTOT $*$ CI IME 
EAFRGY CALCULATIONS - HEATING

136 TF $(N E+1)=T U$

DO $150^{\circ} 1=1$. NE

$J=N E-3+1$

IF $(Z(J) \cdot E Q \cdot 0) R S C L L=$.

IF ( $2(\mathrm{~J}) \cdot E \mathrm{E} .0$.$) GO TO 170$

VSTK $=Z(J) \& E L M /(R H O A * \times N T)$

DSTK =SQRT (1.273*VSTK*XNE/XLT+DU:2

RSDLL =ALDG(DSTK/DO)/(THCS(T(J)) GCRKTL)

$17 C$ RTOT=RFLI+RTUEEL +RSCLL

$X N T U=1.1(R T O T * W F * C P F)$

IF (XNTU.GT.1CC.) EFF $=1$.

IF (XNTU.GT.100.) 6070141

EF F $=1$ - $-E X F(-x(-T U)$

141 IF $(J)=T F(J+1)+E F F *(T(J)-T F(J+1))$

$O F L=W F * C P F *(T F(J+1)-T H(J))$

OTOT $=O F L$

ENER (J)=ENER $(l)+C$ TOT $\&$ C T IME

CALL TKPRCP(TIJ).ENER(J).2(J).J.2.JO)

GC TO IEO

C

TLP ELENENT CALCULATITAS

171 RAXUP=1./2./RTUGEA +2./RSHELA+Z./RNA(J)) +FAXE

RAXLW=1./1. /HTUFEA+1./RSHELA+1./(IFNA $(J)+H N A(J-1)) / 2 \cdot 1)$

QAXUP $=(T A H E-T(J)) / R A X U P$

QAXLW $=(T(J-1)-T(J)) / K A X L W$

QTOT=GAXUF +CAXLWTOINCTOFL

EAER(J)=ENER ( $\lambda)+0 T 01 \div 0 T$ IME

CALL TKPROP(TIJ), EMEK (J).2(J).2.2.J6)

QA XUP $=-O A X L W$

GC 70150

C

BCTTOM ELENENT CALCULATIONS

172 RAXLW=1 $/(2 . / F T U F E A+2 . / R S H E L A+2 . / R M A(J))+R A Y E$

QAXLW=(TANB-I(J) IRAXLW

OTOT=QAXLW TCAYUPTOINSTOFL

EAER(J)=ENER (J) + QTOT $\approx$ TIIME

CALL JKPROP(T(J). ENEF(J):Z(J).2.2.J6)

150 CENTINUE

193 If (CT-EO.15) CO $101 \%$

IF (CT.EG.TO) GO $101 C$

C TI=CTI DTIME

IF (CT.GE.T5.AND.CT.LE.TC) CHGTM=(CT-T5)/3600.

IF (CTI.LT.180C.) GO TO 257

COMT $=(W F+T F(N E+1)+W C C L L T L) /(W F+W C D L L)$

VF ITE $(6.240)$ E.CHGTN.NF, TF (NE+1).WCOLL.CCM)

CT $1=0$.

ZE 7 RE TURN

105 WRITE 6.185$) 15$

C $11=0$.

GC TO 190

1CO WhITE(6.180) 10

150 E $=0$.

$10-15) / 3600$.

WFITE $(6.1 \mathrm{E} 7)$

IF (K7.EG.1) GC TC 160

OC $167 \quad I=1$. NE

TEMPE $L=1.8 \div T(1)-459 \cdot 67$

DF ITE $(6.188)$ I.T(I).Z(1).TF(1). TEMFEL 


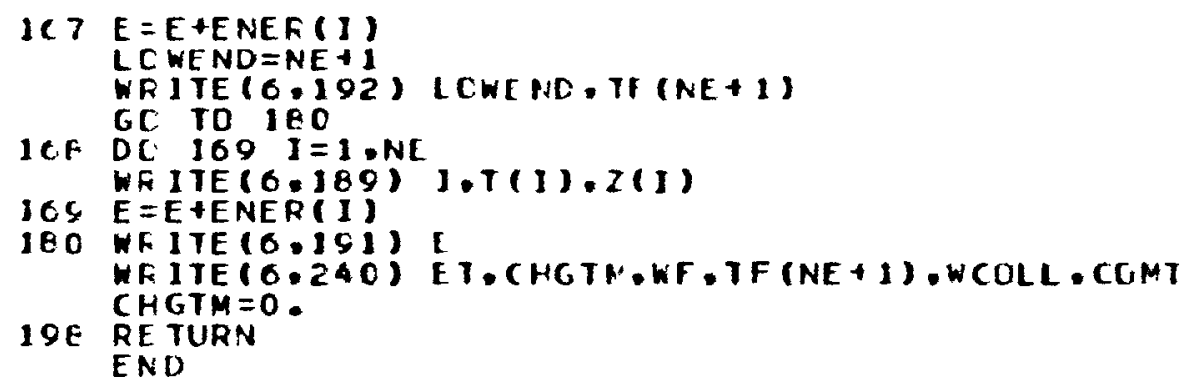


SLIRDUTINE TKTROP(TEMF ENTH.FRAC,M,K2.J6)

C THIS SUBFCUTIAE ESTACLISHES TABLCS OF ELENENT ENTHALPY VS. THE FFACTION DF STLID THIFMKEEP AND TEMFERATURL

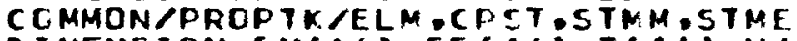

DIMENSION EM(14). EE (j4). T(14).H(14). $(144)$

aCC FORMAT(12)

CCI FCRMATIF10.2.F12.5.F10.6)

202 FORMATI 1HI:5X:" TAELE OF TEMPERATUFE. ENTHALPY ANC SOLID FRACTICN * GF THERMKEEF: $/ /)$

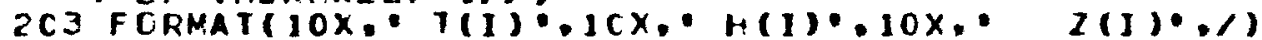

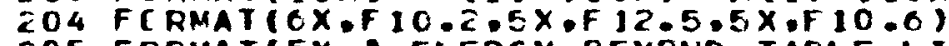

205 FCRMAT(5X: ENERGY PEYOND TABLE LIMITS FCR EMTHE..E10.4) GE TO(1.2) 1.2

1 IF (J6.EQ.2i GG TO 101

WF ITE $(6.202)$

RE AD $(5.200) \times 4$

WF ITE $(6.203)$

$101 J 4=K 4-1$

$J 3=1$

DO $111=1 . K 4$

IF (J6.EC.2) GC TO $10=$

READ(5.201) T(1).H(1).Z(1)

102 CENTINUE

If $(K 2 \cdot E Q \cdot 2)$ GC TO 2

IF $(J 3 \cdot E G \cdot 2)$ GC TO 10

IF IT(I).LT.TENP) GD 1010

ENTH=ELM* (H(1-1)+(TENF-T(1-1))*(H(1)-H(I-1))/(T(1)-1(1-1) )

FFAC $=Z(1)+(T E N P-T(I-1)) *(Z(1)-Z(1-1) /(T(1)-1(1-1)$ $J 3=2$

$10 E M(1)=E L M * H(1)+5 T M M * C F S T * 7(1)$

$E E(I)=E L M * H(I)+5 T M E * C F S T$ T $(I)$

IF $(J 6 \cdot E Q \cdot 2) \quad G 0$ TO 11

WEITE $(6.204) 1(1) . H(1) .2(1)$

11 CONTINUE

RE TURN

2 GC TO(3.4).M

3 IF (ENTH.GT.EM(KA).OF.ENTH.LT.EM(1)) GD TC $25 r$

OC $5 \quad I=1 . J 4$

IF (ENTH.GT EEM(I+1)) CD TO 5

TEMP $=T(I)+(T(1+1)-T(J)) *(E N T H-E N(I)) /(E N(1+1)-E N(I))$

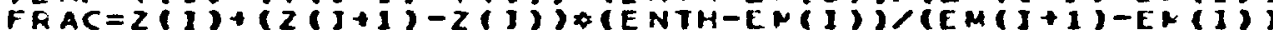

RE TURN

E CCNTINUE

60 TO 250

4 IF (ENTH.GT.EE(KA).OF.ENTH.LT.EE(1)) GD TO $25 \mathrm{C}$

DC $6 \quad I=1 \cdot 14$

It (ENTH.GT.EE(I+1)) GC TO

TF MP $=T(I)+(1(1+1)-7(1))($ ENTH-EE(I) $) /(E E(1+1)-E E(1))$

FRAC $=Z(I)+(Z(1+1)-Z(1))+(E N T H-E E(I)) /(E E(I+I)-E E(I))$

RE TURN

C CONTINUE

IEC TEITE (O.205) ENTH

RE TURN

END 
FUNCTION THCS(T)

C THIS FUNCIION COMPUTES THE THERMAL CONDUCTIVITY CF CDLID THEFNKEET

C AT $250 F$ IT JS NOW A CUMMY FUNCTION WJTH A CONSTART VALUE

$T+C S=1.0800 E-3$

RE TURN

EAD 
FUNCTION THCLIT)

C THIS FUNCTION COMFUTES THE THEFMAL CONDUCTIVITY CF IIOUID THFMKEEF AT 750F. IT IS NCW A DUMMY FUNCTION WITH A CENSTANT VALUE

IA $K J / \mu-S E C-K$

I $C L=1.0207 E-3$

RE TURN

END 
C TLNCTION FF(R) COMFUTLS THE CIRCLLAR TUEE FRICIICA. FACTOR FCF RCUGHNESS RATIO DF - COO12 If (R.GT.2000.) G0 TO I $F F=04 . / R$ RE TURN

1 IF (R.GT.500000.) GO 702 $F F=.3387 \star F *(-.2549)$

RE TURN

$\therefore F F=0.0124$

RE TURN

END 


\section{REFERENCES}

1. Perdue, Donald G. and Larry H. Gordon, "Engineering Evaluation of a Sodium Hydroxide Thermal Energy Storage Module," DOE/NASA/1034-80/7, NASA TM-81417, February 1980.

2. Mellor, Comprehensive Treatise on Inorganic and Theoretical Chemistry, Vol. II, Sup. II, Page 1250.

3. Private communication from T. D. Harrison, Sandia Laboratories, Albuquerque, N.M.

4. Harrison, Thomas D. and William H. McCulloch, "Midtemperature Solar Systems Test Facility (MSSTF) System Test Results, Winter Season," presented at ISES Congress, Atlanta, GA, May 1979.

5. Cohen, Barry M., Richard E. Rice, and Peter E. Rowny, "Development of' a Phase-Change Thermal Storage System Using Modified Anhydrous Sodium Hydroxide for Solar Electric Power Generation," DOE/NASA/0615-79/1, NASA CR-159465, December 1978 . 


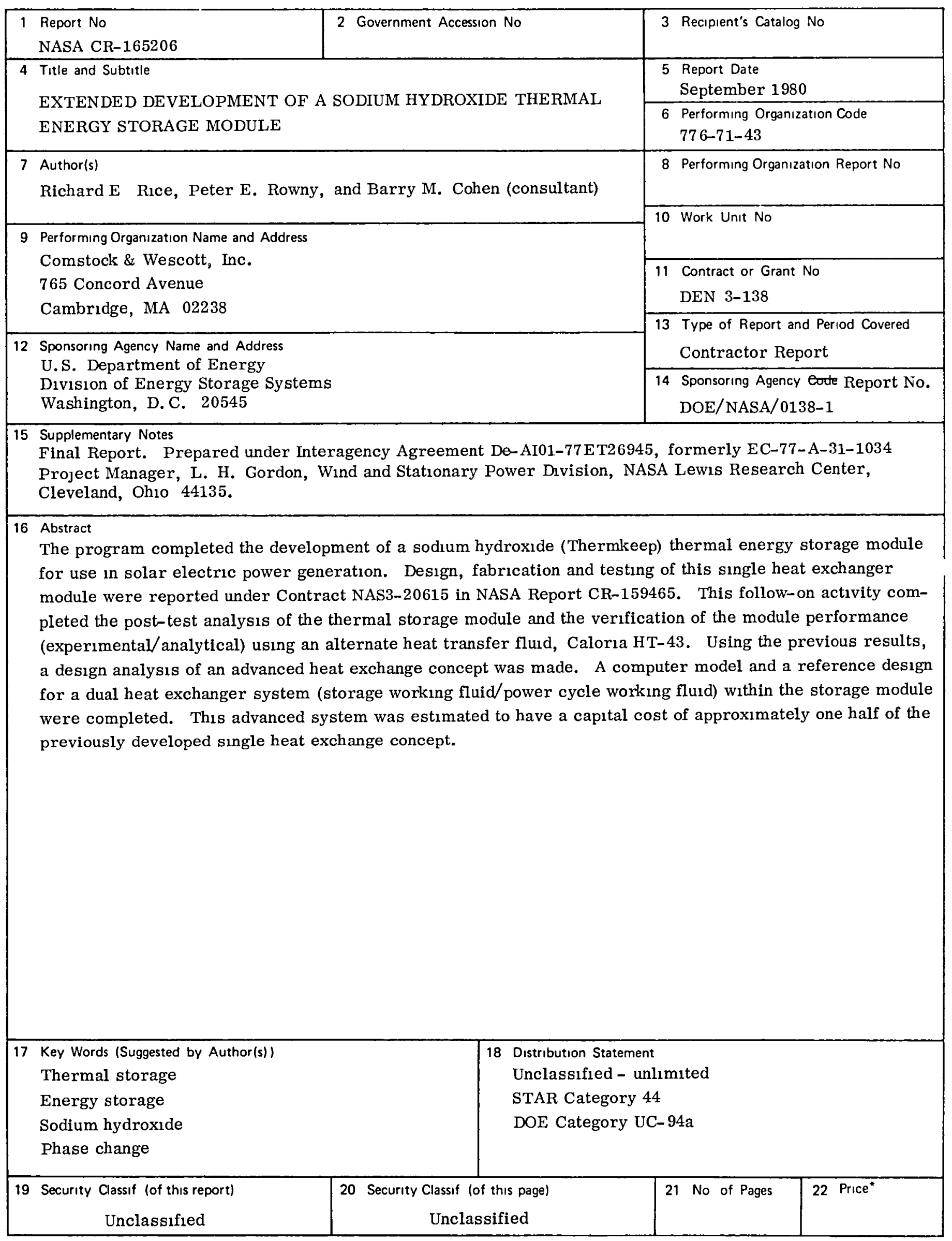

* For sale by the Natıonal Technıcal Informatıon Service, Sprıngfield, Vırgınıa 22161 
End of Document 